\title{
Programmevaluierung Haus der Zukunft \\ 1999 - 2013
}

Evaluierungsbericht

Mag. Dr. Johann Lefenda MA

Mag. Gerlinde Pöchhacker-Tröscher
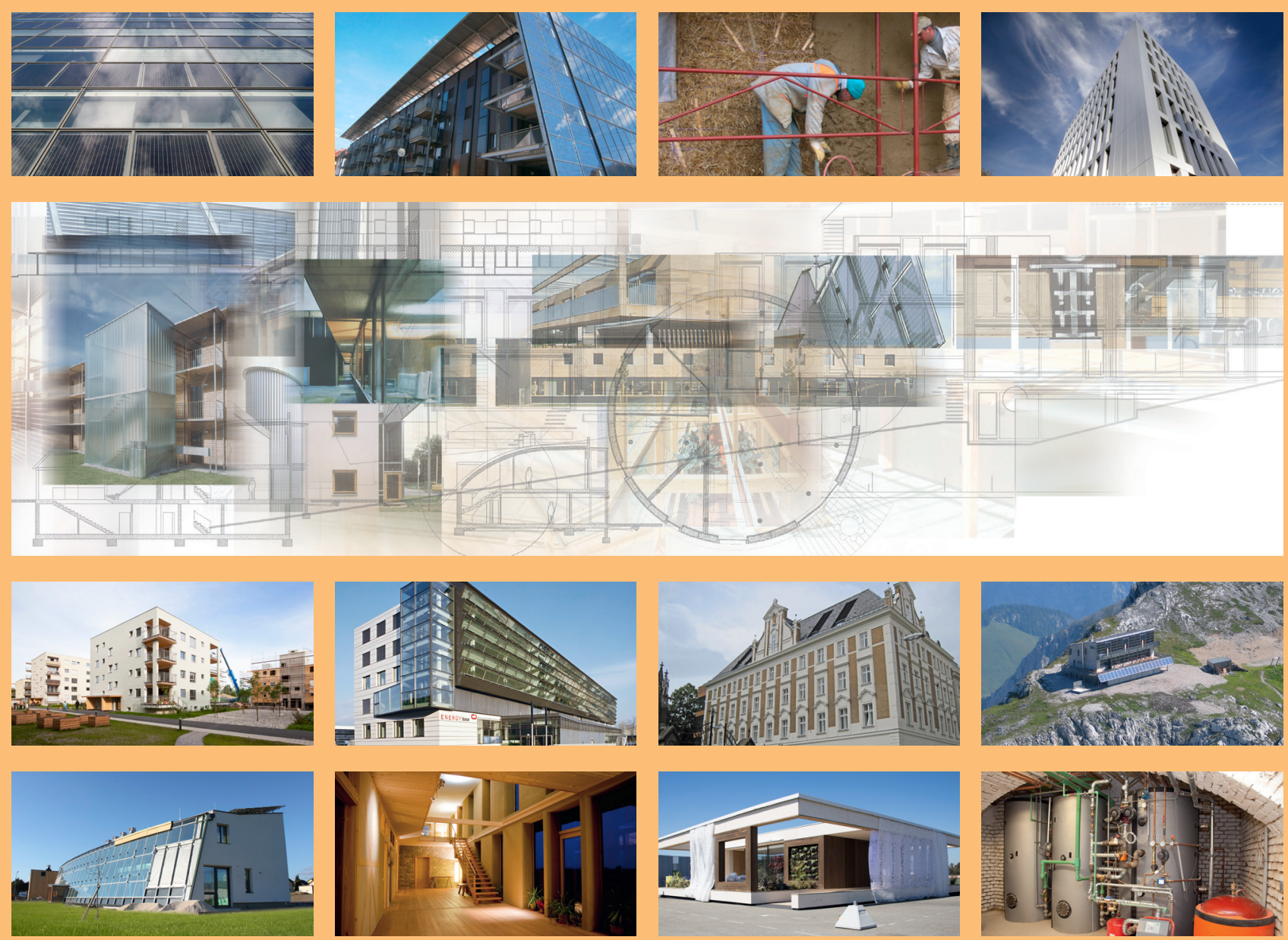
Impressum:

Eigentümer, Herausgeber und Medieninhaber:

Bundesministerium für Verkehr, Innovation und Technologie

Radetzkystraße 2, 1030 Wien

Verantwortung und Koordination:

Abteilung für Energie- und Umwelttechnologien

Leiter: DI Michael Paula

Liste sowie Downloadmöglichkeit aller Berichte dieser Reihe unter http://www.nachhaltigwirtschaften.at 


\section{Programmevaluierung Haus der Zukunft 1999 - 2013}

Evaluierungsbericht

Mag. Dr. Johann Lefenda MA

Mag. Gerlinde Pöchhacker-Tröscher Pöchhacker Innovation Consulting $\mathrm{GmbH}$ 


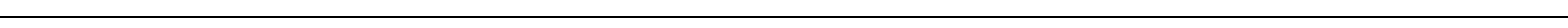




\section{Vorwort}

Der vorliegende Bericht dokumentiert die Ergebnisse eines Projekts aus dem Forschungsund Technologieprogramm Haus der Zukunft des Bundesministeriums für Verkehr, Innovation und Technologie.

Die Intention des Programms ist, die technologischen Voraussetzungen für zukünftige Gebäude zu schaffen. Zukünftige Gebäude sollen höchste Energieeffizienz aufweisen und kostengünstig zu einem Mehr an Lebensqualität beitragen. Manche werden es schaffen, in Summe mehr Energie zu erzeugen als sie verbrauchen („Haus der Zukunft Plus“). Innovationen im Bereich der zukunftsorientierten Bauweise werden eingeleitet und ihre Markteinführung und -verbreitung forciert. Die Ergebnisse werden in Form von Pilot- oder Demonstrationsprojekten umgesetzt, um die Sichtbarkeit von neuen Technologien und Konzepten zu gewährleisten.

Das Programm Haus der Zukunft Plus verfolgt nicht nur den Anspruch, besonders innovative und richtungsweisende Projekte zu initiieren und zu finanzieren, sondern auch die Ergebnisse offensiv zu verbreiten. Daher werden sie in der Schriftenreihe publiziert und elektronisch über das Internet unter der Webadresse www.HAUSderZukunft.at Interessierten öffentlich zugänglich gemacht.

DI Michael Paula

Leiter der Abt. Energie- und Umwelttechnologien

Bundesministerium für Verkehr, Innovation und Technologie 


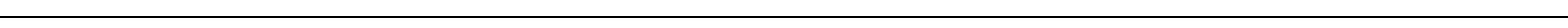




\section{Inhaltsverzeichnis}

Inhaltsverzeichnis 3

$\begin{array}{ll}\text { Kurzfassung } & 6\end{array}$

$\begin{array}{ll}\text { Executive Summary in English } & 13\end{array}$

1 Einleitung 14

$\begin{array}{lll}1.1 & \text { Ausgangssituation } & 14\end{array}$

1.2 Zielsetzung 16

$\begin{array}{lll}1.3 & \text { Evaluierungsdesign und Methodik } & 16\end{array}$

2 INPUT: Förderprogramm Haus der Zukunft 19

$\begin{array}{lll}2.1 & \text { Strategische Einbettung des Programms } & 19\end{array}$

2.2 Programmziele 22

$\begin{array}{lll}2.3 & \text { Programminhalte } & 25\end{array}$

2.4 Programmumsetzung 32

2.5 Relevante Umfeldentwicklungen 34

2.5.1 Internationale und europäische Ebene 34

$\begin{array}{lll}2.5 .2 & \text { Bundesebene } & 40\end{array}$

2.5.3 Länderebene 44

3 OUTPUT: Ergebnisse des Programms 48

3.1 Auswertung der Programmdaten 48

3.1.1 Projektanträge und -genehmigungen 48

3.1.2 Projektförderungen 51

3.1.3 Struktur der Fördernehmer 54

3.2 Demonstrationsprojekte 59

3.3 Leitprojekte 62

3.3.1 Stadtumbau Lehen 65

$\begin{array}{lll}\text { 3.3.2 aspern plus } & 67\end{array}$

$\begin{array}{lll}3.3 .3 \mathrm{e} 80^{\wedge} 3 & 69\end{array}$ 
3.3.4 Innovationsleuchtturm gugler cross media 71

$\begin{array}{lll}3.3 .5 & \text { BIGMODERN } & 73\end{array}$

$\begin{array}{lll}\text { 3.3.6 Gründerzeit mit Zukunft } & 75\end{array}$

3.3.7 Plus-Energie-Bürobau der Zukunft 77

$\begin{array}{lll}\text { 3.3.8 Energy City Graz-Reininghaus } & 79\end{array}$

3.3.9 Gesamthafte Betrachtung der Leitprojekte 82

3.4 Begleitmaßnahmen 84

4 OUTCOME: Wirkung des Programms 89

4.1 Onlinebefragung der Programmteilnehmer 89

$\begin{array}{ll}4.1 .1 & \text { Unternehmen }\end{array}$

$\begin{array}{lll}4.1 .2 & \text { Forschungseinrichtungen } & 99\end{array}$

$\begin{array}{ll}\text { 4.1.3 Sonstige Einrichtungen } & 108\end{array}$

$\begin{array}{lll}4.2 & \text { Interviews mit Schlüsselpersonen } & 110\end{array}$

4.2.1 Interviews mit ausgewählten Projektträgern 110

4.2.2 Interviews mit relevanten Stakeholdern 113

$\begin{array}{ll}\text { 4.2.3 Interviews mit internationalen Experten } & 117\end{array}$

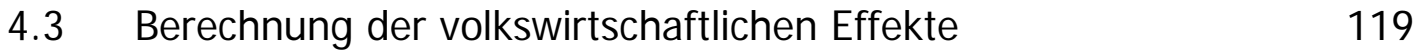

$\begin{array}{lll}4.4 & \text { Wirkungsanalyse } & 123\end{array}$

4.4.1 Bewusstseinsbildung und Sensibilisierung für die Bedeutung und Potenziale Nachhaltigen Bauens in Österreich 125

4.4.2 Berücksichtigung von Nachhaltigkeitskriterien bei Normen, Standards und Förderungen im Baubereich 126

$\begin{array}{lll}\text { 4.4.3 Aufbau und Stärkung der Forschungslandschaft } & 128\end{array}$

4.4.4 Technologie- und Innovationsführerschaft der österreichischen Wirtschaft

4.4.5 Internationale Positionierung Österreichs als Kompetenzträger im Bereich Nachhaltiges Bauen

5 OUTLOOK: Schlussfolgerungen und Empfehlungen

5.1 Schlussfolgerungen 133

5.2 Empfehlungen 135 
Programmevaluierung Haus der Zukunft 1999 - 2013

5.2.1 Vertiefung der Forschungsförderung im Bereich Nachhaltiges Bauen

135

5.2.2 Adressierung von richtungsweisenden Forschungsthemen mit hohem Zukunftspotenzial

5.2.3 Umsetzung von Transfer- und Vernetzungsmaßnahmen im Rahmen geeigneter Instrumente

5.2.4 Impulse von „Haus der Zukunft“ für den Forschungs- und Innovationsstandort Österreich

Literatur- und Quellenverzeichnis

141

Abbildungs- und Tabellenverzeichnis

146 


\section{Kurzfassung}

Der Gebäudesektor spielt eine wesentliche Rolle zur Erreichung der Klimaziele, da Gebäude in der Errichtung und insbesondere im Betrieb in hohem Maß Energie verbrauchen. Um die wirtschaftlichen und technologischen Chancen Österreichs im Gebäudesektor frühzeitig zu erschließen wurde im Jahr 1999 auf Initiative des BMVIT das Programm „Haus der Zukunft" ins Leben gerufen. Dabei wurden in zwei Programmphasen ca. 450 Projekte mit einem Gesamtvolumen von $€ 138$ Mio. mit knapp $€ 80$ Mio. an Förderung unterstützt.

Das BMVIT hat Pöchhacker Innovation Consulting GmbH (P-IC) mit der Durchführung einer Evaluierung der gesamten Programmlaufzeit beauftragt. Der Schwerpunkt lag dabei auf der Darstellung der gesamthaften Wirkungen und Effekte des Programms. P-IC hat hierfür den "Logical Framework"-Ansatz verfolgt, der der klassischen Interventionslogik folgt (Input $\rightarrow$ Output $\rightarrow$ Outcome $\rightarrow$ Outlook). Dazu wurden u.a. eine Onlinebefragung von 84 Projektträgern, 24 leitfadengestützter Gesprächen mit ausgewählten Projektträgern, Stakeholdern und internationalen Experten sowie umfangreiche Dokumenten- und Datenanalysen vorgenommen.

\section{NPUT: Förderprogramm Haus der Zukunft}

Im Mittelpunkt des Programms "Haus der Zukunft" mit einem Fördervolumen von ca. $€ 30$ Mio. in der ersten Phase standen die Entwicklung von Schlüsseltechnologien und erfolgreiche Innovationen für energieeffiziente Gebäude. Wesentliche Bausteine waren die solare Niedrigenergiebauweise, das Passivhaus-Konzept sowie die Nutzung ökologischer Baustoffe und nachwachsender Rohstoffe im Bauwesen. In den Ausschreibungen wurden spezifische inhaltliche Schwerpunkte gesetzt.

Ab 2008 wurde das Programm in einer zweiten Phase unter dem Titel "Haus der Zukunft plus" fortgesetzt und Förderungen von ca. $€ 51$ Mio. ausgeschüttet. Dabei wurden auch die Programmziele und -inhalte weiterentwickelt. Im Fokus stand nun die Zielsetzung, dass die technologischen Voraussetzungen für die Herstellung von „Plus-Energie-Gebäuden“ geschaffen werden sollten. Damit sind Gebäude gemeint, die über den gesamten Lebenszyklus mehr Energie erzeugen als sie verbrauchen.

Dazu wurden F\&E-Projekte unterstützt, um zur Schaffung der technologischen Basis für die Gebäude der Zukunft, zur Überleitung innovativer Technologien und Produkte zur Serien- bzw. industriellen Fertigung und zum Vorantreiben neuer Produktionstechnologien beizutragen. Weiters wurden Demonstrations- und Leitprojekte zur Sichtbarkeit von neuen Technologien und Konzepten sowie intensive Begleitmaßnahmen realisiert, die dem Aufbau einer Fachcommunity, der Bewusstseinsbildung und der Stärkung der internationalen Positionierung Österreichs als Kompetenzträger für Nachhaltiges Bauen im Ausland gewidmet waren. 
Die Programmumsetzung erfolgte in Kooperation unterschiedlicher Einrichtungen: Die Programmverantwortung und -leitung oblag dem BMVIT, die inhaltliche Programm- und Projektbegleitung wurden durch die Österreichische Gesellschaft für Umwelt und Technik (ÖGUT) wahrgenommen. Die operative Abwicklung der Forschungsprojekte erfolgte ab 2004 durch die Forschungsförderungsgesellschaft (FFG). Die Austria Wirtschaftsservice GmbH hatte die Administration der Leitprojekte und die Förderung von Investitionsvorhaben inne. Die zusätzlichen Begleitmaßnahmen wurden durch Beauftragung externer Dienstleister vorgenommen.

Forschung und Innovation im Bereich Nachhaltiges Bauen sind zunehmend in den Fokus gerückt, wie eine Zusammenfassung der Umfeldentwicklungen in den letzten 15 Jahren zeigt. Auf internationaler und europäischer Ebene wurden entsprechende Standards entwickelt und Richtlinien erlassen. In Österreich haben sich die Forschungsausgaben in diesem Bereich deutlich gesteigert und eine Reihe an Institutionen aufgebaut, die über hohe Kompetenzen verfügen. Die Bundesländer haben im Rahmen der Wohnbauförderung mit energetischen Kriterien ein deutliches Signal gesetzt.

\section{OUTPUT: Ergebnisse des Programms}

Grundsätzlich gibt es eine Fülle an Informationen zum Programm und den geförderten Projekten, allerdings liegen aufgrund der langen Laufzeit des Programms keine durchgängigen und einheitlich strukturierten Programmdaten vor, insbesondere zu den ersten drei Ausschreibungen von Haus der Zukunft.

Ab der vierten Ausschreibung von "Haus der Zukunft" wurden 425 Projekte genehmigt, die aus 1.095 Projektanträgen hervorgegangen sind. Die Genehmigungsquote liegt daher bei rd. $40 \%$. Das Interesse an den Programmausschreibungen blieb während der gesamten Laufzeit sehr hoch, wie die kontinuierliche Anzahl an Einreichungen unterstreicht.

Über die Programmlaufzeit ist eine Zunahme der Beteiligung sowohl in absoluten Zahlen wie auch in Relation zur Anzahl der Projekte zu beobachten: In der ersten Programmphase waren 220 Akteure beteiligt (durchschnittlich 1,16 Partner pro Projekt) - in der zweiten Phase insgesamt 696 Akteure (durchschnittlich 2,86 Partner pro Projekt).

In Summe wurden Projekte mit einem Gesamtvolumen von über einer Milliarde Euro beantragt, die beantragte Förderung belief sich insgesamt auf $€ 306$ Mio. Es konnten davon Projekte mit einem Gesamtvolumen von $€ 138$ Mio. mit einer Förderung in Höhe von knapp $€ 80$ Mio. unterstützt werden. Dies sind deutliche Zeichen für die Potenziale von Forschung und Innovation im Bereich Nachhaltiges Bauen in Österreich. 
Die Auswertung nach dem Organisationstyp zeigt, dass insbesondere die Unternehmen mit diesem Programm erreicht werden konnten. In der ersten Programmphase machten sie rund die Hälfte aller Begünstigten aus, in der zweiten Programmphase rund ein Drittel. Zudem waren sie vielfach in Forschungskooperationen vertreten, welche in der zweiten Programmphase an Gewicht zugenommen haben. Insbesondere wissensbasierte Dienstleistungsunternehmen haben sich stark in Haus der Zukunft-Projekten engagiert (zB Architektur- und Planungsbüros). Die Bauindustrie im engeren Sinn hat sich nur punktuell an Projekten beteiligt.

Bereits ab der dritten Ausschreibung wurden auch Demonstrationsprojekte gefördert, bei denen die praktische Umsetzung innovativer Konzepte, Ansätze und Technologien im Vordergrund stand. Damit sollte die Praxistauglichkeit neuer Entwicklungen unter Beweis gestellt und der erzielbare Energieeinsparungseffekt aufgezeigt werden. Die 68 realisierten Demonstrationsprojekte haben vielfach eine hohe nationale und internationale Reputation verfahren und wurden mit zahlreichen Preisen ausgezeichnet.

Im Rahmen der ersten Ausschreibung der zweiten Programmphase im Jahr 2009 wurden Leitprojekte gestartet. Dabei handelte es sich um ein neues Projektformat, das aus einem zusammenhängenden Bündel von Einzelprojekten („Subprojekte“) bestand, die - integriert in eine klar definierte Strategie - jeweils ein konkret beschriebenes Ziel verfolgten und von einem gemeinsamen Gesamtprojektmanagement koordiniert wurden. Die Leitprojekte waren vorrangig den Themen Plus-Energie-Siedlungen und Sanierung gewidmet:

* Stadtumbau Lehen: Nachhaltige Stadtteilentwicklung in Salzburg

* aspern plus: Gesamtkonzept und Demonstrationsgebäude für ein energieoptimiertes Stadterweiterungsgebiet

* e80^3-Gebäude: Vorgefertigte Dach- und Energiefassadenelemente mit integrierter Haustechnik

* Leuchtturm Gugler: Ökologisch und energetisch optimiertes Betriebsgebäude

* BIGMODERN: Sanierung von öffentlichen Gebäuden nach Passivhausstandard

* GdZ - Gründerzeit mit Zukunft: Energieoptimierte Sanierung von historischen Gebäuden, zT unter Denkmalschutz

* Plus-Energie-Bürobau der Zukunft: Gesamthafte Analyse und Optimierung des Energieverbrauchs in einem Bürogebäude der TU Wien

* ECR Energy City Graz Reininghaus: Gesamtenergiekonzept und Demonstrationsgebäude im Stadtentwicklungsgebiet Graz-Reininghaus 
Das Instrument der Leitprojekte war ein besonders innovativer Ansatz in der Forschungsförderung und eröffnete die Möglichkeit, komplexe Themenstellungen in ihrer Breite zu behandeln und den Schritt von der Forschung in die praktische Anwendung zu gehen. Damit wurde Forschung und Innovation im Bereich Nachhaltigen Bauens sichtbar und begreifbar gemacht, was wiederum wesentlich war für die Stärkung der internationalen Sichtbarkeit Österreichs. Die Leitprojekte wurden und werden vielfach von internationalen Delegationen besucht und erweisen sich als "Türöffner" für internationale Forschungsprojekte und Unternehmenskooperationen.

Neben der Umsetzung von Leitprojekten liegt ein weiteres Alleinstellungsmerkmal von Haus der Zukunft in der starken Betonung von Begleitmaßnahmen. Diese Maßnahmen stehen in unmittelbarem Zusammenhang mit den Programmzielen, die nicht nur die Technologieentwicklung sondern auch den Aufbau einer entsprechenden Community, die Forcierung der Anwendung und Marktdurchdringung innovativer Lösungen, den Kompetenzaufbau uäm. adressierten. Diese Aktivitäten waren gerade für die sehr heterogene Bauszene, die jedoch in hohem Maß auf das Zusammenspiel unterschiedlicher Akteure angewiesen ist, von großer Bedeutung.

\section{OUTCOME: Wirkung des Programms}

Der vierte Abschnitt des Evaluierungsberichts beleuchtet den OUTCOME. Damit sind die indirekt erzielten Wirkungen des Förderprogramms erfasst, die sich aus den direkten Ergebnissen wie insbesondere den geförderten Projekten ergeben haben. Um diese Wirkungen zu erfassen, wurde zunächst eine Onlinebefragung unter allen Programmteilnehmern durchgeführt.

37 Unternehmen haben an der Onlinebefragung teilgenommen, davon sind 23 in der Dienstleistungsbranche tätig. Mehr als die Hälfte der befragten Unternehmen hat bei dem Haus der Zukunft-Projekt zum ersten Mal an einem geförderten F\&EProjekt teilgenommen. Die Unternehmen konnten mit den Projekten vor allem spezifisches Fachwissen gewinnen. In $2 / 3$ der Unternehmen wurde ein neues Produkt oder eine neue Dienstleistung entwickelt. Jedes dritte Unternehmen hat in weiterer Folge neue Arbeitsplätze geschaffen. Fast alle Unternehmen haben nach dem Projekt an dem Thema gearbeitet, wenngleich in unterschiedlicher Form. Mehr als $3 / 4$ der Unternehmen wollen auch in Zukunft Forschung und Innovation betreiben - erforderlich sind dafür neben Finanzmitteln für Forschung und Innovation auch die Vernetzung mit Partnern und qualifizierte Mitarbeiter.

Weiters haben sich $\mathbf{4 0}$ Forschungseinrichtungen an der Onlinebefragung beteiligt. Deren Haus der Zukunft-Projekte waren meist in der Grundlagenforschung bzw. im Bereich der Begleitmaßnahmen sowie der Entwicklung innovativer Bauund Sanierungskonzepte angesiedelt. Mehr als die Hälfte der Forschungseinrichtungen hätte ohne die Förderung nicht an dem Thema forschen können. Über 80 
geben an, dass das Projekt über die Laufzeit hinweg nachhaltige Wirkung auf die Forschungseinrichtung hatte. Diese liegt vorrangig in drei Bereichen: Durch das Projekt konnte wissenschaftliches Personal aufgenommen bzw. gehalten werden. Weiters haben die Projekte maßgeblich die Herausbildung von Forschungsschwerpunkten unterstützt. Nicht zuletzt haben die Haus der Zukunft-Projekte auch zur Schärfung und Verbreitung des Profils der wissenschaftlichen Einrichtungen beigetragen, die sich etwa in Nachfolgeprojekten im Rahmen von europäischen und internationalen Programmen niederschlagen. Die Begleitmaßnahmen waren aus Sicht der Forschungseinrichtungen von zentraler Bedeutung, insb. die Publikationen, die Online-Plattform www.hausderzukunft.at (wo u.a. alle Projektberichte verfügbar sind) und die themenspezifischen Workshops.

Die dritte Gruppe der Befragten stellten sonstige Einrichtungen dar (öffentliche Einrichtungen, Vereine u.dgl.). Die unmittelbaren Wirkungen, die durch das Projekt generiert worden sind, liegen in erster Linie in der Gewinnung von spezifischem Fachwissen, der Intensivierung bestehender und Gewinnung neuer Kontakte zu Partnerorganisationen sowie in der Steigerung an Sichtbarkeit in der Fachcommunity. Haus der Zukunft in seiner Gesamtheit hat aus Sicht der sonstigen Einrichtungen maßgeblich zur Bewusstseinsbildung und Sensibilisierung für Forschung und Innovation in der österreichischen Bauwirtschaft, der internationalen Profilierung Österreichs, der Senkung des Energieverbrauchs im Gebäudebereich, der Sichtbarmachung der Potenziale Nachhaltigen Bauens und der Intensivierung des Wissenstransfers beigetragen.

In Ergänzung zur Onlinebefragung wurden mit ausgewählten Personen vertiefende Interviews geführt. Dabei wurden drei Gruppen abgebildet:

* Ausgewählte Projektträger, die aufgrund ihrer Funktion einen besonders tiefen Einblick in die Programmumsetzung und -wirkungen haben

* Stakeholder der österreichischen Forschungs- und Wirtschaftscommunity im Themenfeld Nachhaltiges Bauen

* Internationale Experten, die Haus der Zukunft aus einer Außensicht einschätzen können

Der Tenor der Gespräche war eindeutig positiv: Haus der Zukunft hat wesentliche und frühzeitige Impulse zum Aufbau von Kompetenzen österreichischer Akteure im Bereich Nachhaltiges Bauen und deren Vernetzung gesetzt. Weiters wurde durch die kontinuierliche Weiterentwicklung des Programms in Hinblick auf die Themenschwerpunkte und die Instrumente das Niveau der Projekte kontinuierlich gesteigert. Österreich konnte sich somit zu einem international beachteten Vorreiter in diesem Themenfeld etablieren. Vereinzelt wurde in den Gesprächen auch Kritik geäußert und Verbesserungspotenziale, etwa in Hinblick auf die Ansprache neuer Zielgruppen, angesprochen/gesehen. 
Eine Berechnung der volkswirtschaftlichen Effekte des Programms, die vom Ökonomen Univ.-Prof. Dr. Friedrich Schneider (Johannes Kepler Universität Linz) durchgeführt wurde, hat die Wirkungen des Programms auf die österreichische Volkswirtschaft aufgezeigt: In Summe wurden über die gesamte Programmlaufzeit hinweg $€ 204,202$ Mio. an zusätzlichem Bruttoinlandsprodukt, ein zusätzliches Masseneinkommen von $€ 88,236$ Mio. und 1.643 Arbeitsplätze ausgelöst.

Zusammenfassend zeigt die Wirkungsanalyse vielfältige positive Effekte des Programms auf, die insbesondere in folgenden Dimensionen liegen:

Bewusstseinsbildung und frühzeitige Sensibilisierung für die Bedeutung und Potenziale Nachhaltigen Bauens in Österreich: Durch das Programm wurde Stakeholdern und der breiten Öffentlichkeit aufgezeigt, welchen Stellenwert das Gebäude für die Erreichung von Klimazielen hat, welche Einsparungsmöglichkeiten realisierbar sind und welcher Mehrwert von nachhaltigen Gebäuden für die Bewohner und Benutzer einhergeht. Auch wurden die Marktchancen für österreichische Anbieter in diesem Segment aufgezeigt.

* Berücksichtigung von Nachhaltigkeitskriterien bei Normen, Standards und Förderungen im Baubereich: Haus der Zukunft hat die technischen Grundlagen für die Entwicklung von Baustandards geschaffen und nicht zuletzt durch die Demonstrations- und Leitprojekte - die Verankerung dieser Standards in Regelwerken wie der Wohnbauförderung vorangetrieben

* Nachhaltiger Aufbau und Stärkung der Forschungslandschaft: Das Programm hat über einen langen Zeitraum ein Thema adressiert und kontinuierlich neue Aspekte angesprochen. Diese Langfristigkeit und Fokussierung hat den Aufbau und die Stärkung von Forschungsstrukturen in Österreich im Themenfeld Nachhaltiges Bauen mit internationaler Strahlkraft ermöglicht.

* Technologie- und I nnovationsführerschaft der österreichischen Wirtschaft: Österreich hat sich in den vergangenen 15-20 Jahren zu einem „Frontrunner" im Bereich Nachhaltiges Bauen entwickelt - sowohl in Hinblick auf die wissenschaftlichen Kompetenzen wie auch die spezialisierten Unternehmen, die auf nationalen und internationalen Märkten reüssieren. Insbesondere wissensbasierte Dienstleister sind hierbei zu erwähnen, da sie eine Schlüsselrolle im Planungs- und Bauprozess einnehmen und als Türöffner für andere Gewerke fungieren können.

* Internationale Positionierung Österreichs als Kompetenzträger im Bereich Nachhaltiges Bauen: Haus der Zukunft hat erhebliche Wirkungen in Hinblick auf die internationale Positionierung Österreichs als Kompetenzträger im Bereich Nachhaltiges Bauen entfaltet. Das Programm wurde vielfach als Best Practice-Beispiel vorgestellt, u.a. im Rahmen einer Enquete im Europäischen Parlament. Zudem wurden viele Gebäude, die daraus hervorgegangen sind, mit internationalen Preisen ausgezeichnet. 
OUTLOOK: Schlussfolgerungen und Ausblick

Die Evaluierung zeigt, dass Haus der Zukunft sehr deutliche und nachhaltige Wirkungen initiiert hat. Das Programm war ein maßgeblicher Stimulus, um die Technologiekompetenzen von Forschungsakteuren und die Marktposition österreichischer Unternehmen im Bereich des Nachhaltigen Bauens zu entwickeln und zu vertiefen.

Die Evaluierung zeigt ein herausragend positives Ergebnis. Das Programm war sowohl in Hinblick auf die thematische Ausgestaltung wie auch die Wahl der Instrumente, die operative Umsetzung und die erzielten Wirkungen beispielgebend für praxisnahe, zukunftsweisende FTI-Programme. Die Begleitmaßnahmen sind hierbei besonders zu erwähnen. Die Zielsetzungen der beiden Programmphasen konnten jeweils erreicht werden.

Daher wird eine Fortsetzung der Aktivitäten zur Unterstützung von Forschung und Innovation im Themenfeld Nachhaltiges Bauen angeregt. Diese Weiterführung kann im Rahmen bestehender Initiativen und Programme sowie durch Vernetzungs- und Kooperationsaktivitäten erfolgen. Folgende Themenfelder könnten dabei adressiert werden:

Vertiefung der Forschungsförderung im Bereich Nachhaltiges Bauen: zB Fortsetzung der Adressierung von Forschungsfragen in Bezug auf Einzelgebäude im Rahmen des Programms "Stadt der Zukunft“, Weiterführung der praktischen Umsetzung von Forschungsergebnissen im Rahmen von Demonstrations- und Leitprojekten etc.

* Adressierung von richtungsweisenden Forschungsthemen mit hohem Zukunftspotenzial: zB Verfahren und Technologien für die nachhaltige Sanierung, leistbares nachhaltiges Wohnen, „Digitales Bauen“, Lüftung und Kühlung, Bauteilaktivierung zur lokalen thermischen und energetischen Speicherung, ökologisches Bauen etc.

* Umsetzung von Transfer- und Vernetzungsmaßnahmen im Rahmen geeigneter I nstrumente: $z B$ Weiterentwicklung der Online-Plattform, aktive Unterstützung des Wissenstransfers, programmübergreifende Transfermaßnahmen uäm.

* I mpulse von „Haus der Zukunft“ für den Forschungs- und I nnovationsstandort Österreich: Entwicklung einer „Österreichischen Bauinnovationsstrategie", gezielte internationale Positionierung Österreichs als Kompetenzregion für Nachhaltiges Bauen, Vergabe öffentlicher Bauaufträge nach den Lebenszykluskosten, stärkere Kapitalisierung von Forschungsergebnissen, regelmäßige und strukturiertes Trendscreening- und Foresight-Aktivitäten zur Identifikation von neuen internationalen Trends mit hohem Marktpotenzial etc. 


\section{Executive Summary in English}

The buildings sector plays a significant role in achieving climate objectives because the construction and in particular in the use operation of buildings is highly relevant in terms of energy and sustainability. In order to address explore the economic and technological opportunities in Austria concerning sustainable building, the funding program "Building of Tomorrow" was established by the federal Austrian ministry for Transport, Innovation and Technology (BMVIT). Launched in 1999 and established in two program stages, about 450 projects with a total project volume of $€ 138 \mathrm{~m}$ were funded with $€ 80 \mathrm{~m}$.

Pöchhacker Innovation Consulting (P-IC) was entrusted with the implementation of a comprehensive evaluation of the entire program with main focus on the description of the overall program effects. For this purpose, based on the logical framework approach, the relevant documents and data were analyzed and evaluated. Furthermore, an online survey was conducted among all project managers, in which 84 project managers participated. In addition, 24 interviews with selected project managers as well as national and international experts were conducted.

"Building of Tomorrow" addressed a highly future-oriented topic at an early stage and continued to develop in terms of the thematic priorities and instruments. In addition to numerous $R \& D$ projects, 68 demonstration and 8 key projects and comprehensive accompanying measures were set, which contributed significantly to the establishment of a professional community of Austrian key players, experts and companies in the sector.

The economic effect of the program amounts to a total of $€ 204 \mathrm{~m}$ of the additional gross domestic product, an additional mass income of $€ 88 \mathrm{~m}$ and as well as 1.643 newly created or secured jobs.

The program shows multiple positive effects: The awareness of the importance and potential of sustainable building was raised, sustainability criteria were considered within the relevant norms and standards, research institutions were set up resp. strengthened, and the international position of Austria as a technology and innovation leader in sustainable building was significantly improved.

Therefore, a continuation of the activities to promote research and innovation in sustainable building is recommended. Research funding in sustainable building should constantly be maintained, focusing on current issues (e.g. population growth in emerging countries). Research topics with high potential for the future should be specifically addressed. Transfer measures should be set through appropriate instruments, and further inputs of the program "Building of Tomorrow" for the research and innovation policy in Austria should be taken up. 


\section{$1 \quad$ Einleitung}

\subsection{Ausgangssituation}

Gebäude sind nicht nur ein Nutzgegenstand, sondern ein wesentlicher Aspekt des menschlichen Lebens. Rund $90 \%$ seiner Zeit verbringt der Durchschnittsmensch innerhalb von Gebäuden. Gebäude haben auch - eine Seltenheit in der heutigen Zeit - eine sehr hohe Lebens- und Nutzungsdauer von 40 bis 100 Jahren. Häuser, die 2016 gebaut werden, könnten also durchaus im Jahr 2100 noch stehen.

Zur Erreichung internationaler und nationaler Klimaziele ist eine Steigerung der Energieeffizienz und des Einsatzes erneuerbarer Energien erforderlich. Der Gebäudebereich ist dafür in besonderer Weise relevant, da sowohl im Bau wie auch im Betrieb von Gebäuden Energie in hohem Maß eingesetzt wird. Seit den 1960er Jahren hat sich der Gebäudebestand in Österreich verdoppelt. Pro Jahr werden in Österreich etwa 40.000 Wohnungen neu errichtet. Über $1 \%$ der mehr als 3 Millionen bestehenden Wohnungen werden jährlich energetisch saniert.

Die Bauwirtschaft ist auch ein beträchtlicher ökonomischer Faktor in Österreich. Laut Leistungs- und Strukturdaten der Statistik Austria (vorläufige Werte für 2014) sind unmittelbar im Bausektor über 33.000 Unternehmen mit 286.000 Beschäftigten tätig. Die Umsatzerlöse beliefen sich im Jahr 2014 auf $€$ 42,4 Mrd. Damit umfasst die Bauwirtschaft rund $10 \%$ aller Betriebe und Beschäftigten und einen Anteil von $6,5 \%$ an den gesamten Betriebserlösen.

Gebäude sind von zentraler Bedeutung für den Energie- und Ressourcenverbrauch. $25 \%$ der mineralischen Stoffflüsse und $50 \%$ des Abfallaufkommens sind dem Gebäudebereich zuzuordnen. Nahezu $\mathbf{4 0}$ \% des Endenergieeinsatzes entfallen auf den Bereich Raumwärme, der wesentlich von der Gebäudequalität bestimmt wird. Darüber hinaus ist die Gestaltung der bebauten Umwelt in besonderem Maße für die Lebensqualität der Bewohner von Bedeutung.

Nachhaltige Gebäudetechnologien sind ein zunehmend bedeutsames Wirtschafts- und Innovationsfeld, da der Klimawandel, das weltweite Bevölkerungswachstum und ökonomische Prosperität den Bedarf nach energieeffizienten und nachhaltigen Baulösungen weiter ansteigen lassen werden. Aus ökologischen und ökonomischen Motiven ist es erforderlich, neue Gebäude in nachhaltiger Form zu errichten und mit Grund und Boden verantwortungsvoll für kommenden Generationen umzugehen.

Um die wirtschaftlichen und technischen Chancen Österreichs im Bereich des Nachhaltigen Bauens zu erschließen, bedarf es einer gezielten Stimulierung der relevanten Akteure, um kritische Massen aufzubauen, Forschungs- und Entwick- 
lungsprojekte voranzutreiben und durch Demonstrationsprojekte die Möglichkeiten innovativer Technologien in der Praxis aufzuzeigen. Das Bundesministerium für Verkehr, Innovation und Technologie (BMVIT) hat diesen Zukunftstrend frühzeitig erkannt und bereits 1999 das Programm Haus der Zukunft ${ }^{1}$ ins Leben gerufen. Es zielte darauf $a b$, die Entwicklung und Umsetzung zukunftsweisender Konzepte und Technologien für die Gebäude der Zukunft zu unterstützen und zu fördern.

Im Mittelpunkt des Programms standen die Entwicklung von Schlüsseltechnologien und erfolgreiche Innovationen für energieeffiziente Gebäude. Wesentliche Bausteine waren die solare Niedrigenergiebauweise, das Passivhaus-Konzept sowie die Nutzung ökologischer Baustoffe und nachwachsender Rohstoffe im Bauwesen. In den zahlreichen Ausschreibungen wurden spezifische inhaltliche Schwerpunkte gesetzt.

Dazu wurden Forschungs-, Entwicklungs-, Investitions- und Demonstrationsvorhaben sowie Leitprojekte unterstützt, um u.a. zur Schaffung der technologischen Basis für die Gebäude der Zukunft, zur Überleitung innovativer Technologien und Produkte zur Serien- bzw. industriellen Fertigung und/oder zum Vorantreiben neuer Produktionstechnologien beizutragen. Weiters wurden Leuchtturmprojekte zur Sichtbarkeit von neuen Technologien und Konzepte sowie Aktivitäten zur Verstärkung des internationalen Know-how-Transfers und des Aufbaus von Humanressourcen forciert.

In Summe wurden 432 Projekte mit einem Gesamtvolumen von $€ 138$ Mio. mit knapp $€ \mathbf{8 0}$ Mio. an Förderung unterstützt, was einen wesentlichen technologischen Schub mit " $\mathrm{CO}_{2}$-Relevanz" für die österreichische Bauwissenschaft und wirtschaft darstellte. Darüber hinaus wurden umfangreiche Begleitmaßnahmen durchgeführt, um den Aufbau einer Fachcommunity zu unterstützen, die Verbreitung der Ergebnisse von Forschungs- und Innovationsprojekten voranzutreiben und die Öffentlichkeit über die Chancen und Potenziale von Nachhaltigem Bauen zu informieren.

Ferner hat das Programm - direkt und indirekt - maßgeblich dazu beigetragen, die internationale Positionierung Österreichs als Kompetenzträger im Bereich Nachhaltiges Bauen zu stärken. Dies schließt die Hersteller von Baustoffen und Baukomponenten ebenso mit ein wie wissensbasierte Dienstleister des Bausektors und die universitären und außeruniversitären Forschungseinrichtungen im Gebäudebereich.

1 Aus Gründen der Lesbarkeit steht die Bezeichnung Haus der Zukunft für die beiden Programmphasen "Haus der Zukunft" und "Haus der Zukunft plus". 


\subsection{Zielsetzung}

Die Abteilung für Energie- und Umwelttechnologien des Bereichs Innovation im BMVIT, welche das Programm Haus der Zukunft initiiert, finanziert und strategisch gesteuert hat, beauftragte Pöchhacker Innovation Consulting (P-IC) mit der Durchführung einer gesamthaften Evaluierung des Programms Haus der Zukunft über die Laufzeit von 1999 bis 2013.

Mit der Evaluierung soll die generelle Wirkung des Programms über die gesamte Dauer hinweg dargestellt werden. Dabei sollen nicht nur die unmittelbaren Ergebnisse des Programms im Sinne von Forschungs- und Innovationsprojekten, sondern auch die indirekten Wirkungen, etwa durch die strategischen Begleitmaßnahmen, berücksichtigt werden. Darauf aufbauend sollen Schlussfolgerungen und Anregungen für die Gestaltung von künftigen Forschungsprogrammen durch die zuständige Abteilung im BMVIT abgeleitet werden.

\subsection{Evaluierungsdesign und Methodik}

Als grundlegendes Konzept für die vorliegende Evaluierung wurde der "Logical Framework"-Ansatz gewählt. Dieser erlaubt es, verschiedene methodische Ansätze im Sinne einer ganzheitlichen Evaluierung zu kombinieren.

Im Kern folgt der Logical Framework-Ansatz der klassischen Interventionslogik:

Definierte Ziele sollen durch eine Intervention der öffentlichen Hand erreicht werden.

* Die Umsetzung von Interventionsaktivitäten bildet dazu einen I nput - im konkreten Fall besteht dieser Input in den Förderungen und Aktivitäten im Rahmen von Haus der Zukunft.

* Diese bewirken einen unmittelbar sichtbaren Output wie bspw. die Durchführung von Forschungsvorhaben oder die Realisierung von innovativen Bauprojekten.

* Darüber hinaus bewirken die Maßnahmen eine indirekte Wirkung, die als Outcome oder Impact bezeichnet werden kann und jene Effekte meint, die aus dem Output heraus generiert werden.

* Im Outlook werden Schlussfolgerungen und Empfehlungen in Form eines Ausblicks festgehalten, die wiederum als inhaltliche Basis für einen neuen Input herangezogen werden können, sodass sich der Kreislauf schließt. 
Abbildung 1: Evaluierungsdesign

\begin{tabular}{|c|c|c|c|c|c|}
\hline (1) & Ziele & $\begin{array}{l}\text { INPUT: } \\
\text { Programm }\end{array}$ & $\begin{array}{l}\text { OUTPUT: } \\
\text { Ergebnisse }\end{array}$ & $\begin{array}{l}\text { OUTCOME: } \\
\text { Wirkungen }\end{array}$ & $\begin{array}{c}\text { OUTLOOK: } \\
\text { Empfehlungen }\end{array}$ \\
\hline \multirow{8}{*}{ 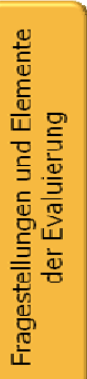 } & \multirow{2}{*}{$\begin{array}{l}\text { Ziele des Programms } \\
\text { Ziele im Zeitverlauf }\end{array}$} & \multirow{2}{*}{$\begin{array}{l}\text { Eingesetzte Ressourcen } \\
\text { Ausschreibungen }\end{array}$} & $\begin{array}{l}\text { Geförderte Projekte, } \\
\text { Demonstrationsproj. }\end{array}$ & Zielerreichung & \multirow{3}{*}{$\begin{array}{l}\text { Programmwirkung ggü. } \\
\text { Dienstleister, Techno- } \\
\text { logiehersteller, Bauträ- } \\
\text { ger, Entscheidungs- } \\
\text { träger, Wirtschaft }\end{array}$} \\
\hline & & & Leitprojekte & \multirow{2}{*}{$\begin{array}{l}\text { Entwicklung Techno- } \\
\text { logiestärkefelder }\end{array}$} & \\
\hline & Zielgruppen, -sektoren & \multirow{2}{*}{$\begin{array}{l}\text { Instrumentenmix, } \\
\text { Begleitmaßnahmen }\end{array}$} & \multirow{2}{*}{ Publikationen } & & \\
\hline & \multirow{5}{*}{ Kohärenz der Ziele } & & & $\begin{array}{l}\text { Entwicklung Forsch. } \\
\text { bereiche/-community }\end{array}$ & \multirow{3}{*}{$\begin{array}{l}\text { Wirkungen von Strate- } \\
\text { gischen Überlegungen } \\
\text { \& Programmbegleiten- } \\
\text { den Aktivitäten }\end{array}$} \\
\hline & & $\begin{array}{l}\text { Strategisches Pro- } \\
\text { gramm-Management }\end{array}$ & \multirow[t]{4}{*}{$\begin{array}{l}\text { Veranstaltungen, } \\
\text { Vernetzung, Info etc. }\end{array}$} & \multirow{2}{*}{$\begin{array}{l}\text { Erfolg / Zweckmäßig- } \\
\text { keit der Maßnahmen }\end{array}$} & \\
\hline & & \multirow{2}{*}{$\begin{array}{l}\text { Kohärenz Ziele und } \\
\text { Maßnahmen }\end{array}$} & & & \\
\hline & & & & \multirow{2}{*}{$\begin{array}{l}\text { Volkswirtschaftliche } \\
\text { Effekte }\end{array}$} & \multirow{2}{*}{$\begin{array}{l}\text { Schlussfolgerungen \& } \\
\text { Empfehlungen }\end{array}$} \\
\hline & & Begleitmaßnahmen & & & \\
\hline \multirow{5}{*}{ 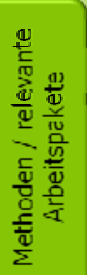 } & $\begin{array}{l}\text { Dokumentenanalyse } \\
\text { (AP1) }\end{array}$ & $\begin{array}{l}\text { Dokumenten- } \\
\text { Datenanalyse (AP1) }\end{array}$ & $\begin{array}{l}\text { Dokumenten- } \\
\text { Datenanalyse (AP1) }\end{array}$ & Umfeldanalyse (AP1) & $\begin{array}{l}\text { Entwurf Bericht und } \\
\text { Empfehlungen (AP6) }\end{array}$ \\
\hline & Datenanalyse (AP1) & \multirow[t]{4}{*}{ Tiefeninterviews (AP3) } & Tiefeninterviews (AP3) & Online-Befragung (AP2) & \multirow{2}{*}{$\begin{array}{l}\text { Reflexionsworkshop } \\
\text { (AP6) }\end{array}$} \\
\hline & \multirow{3}{*}{ Umfeldanalyse (AP1) } & & \multirow{3}{*}{ Leitprojekte (AP4) } & Tiefeninterviews (AP3) & \\
\hline & & & & Leitprojekte (AP4) & $\begin{array}{l}\text { Finalisierung Evaluie- } \\
\text { rungsberlcht (AP6) }\end{array}$ \\
\hline & & & & Synthese (AP5) & \\
\hline
\end{tabular}

Quelle: Eigene Darstellung

Diesem Ansatz entsprechend wurde der Evaluierungsprozess in sechs Arbeitspakete untergliedert und im Zeitraum September 2015 bis März 2016 durchgeführt:

1. Dokumenten-, Daten- und Umfeldanalyse: Analyse der umfangreichen Programmdokumente (zB Ausschreibungsleitfäden, Zwischenevaluierung, Projektberichte u.dgl.), Auswertung von Programmdaten und Analyse relevanter Umfeldentwicklungen

2. Online-Befragung der Programmteilnehmer: Befragung von 421 Programmteilnehmern mittels Online-Befragung

3. Experteninterviews: Durchführung und Auswertung von 24 leitfadengestützen Gesprächen mit ausgewählten Projektträgern, Stakeholdern und internationalen Experten

4. Dokumentation und Analyse der Leitprojekte: Detaillierte Dokumentation der acht Leitprojekte sowie Durchführung und Auswertung von Interviews mit den Leitprojekt-Managern

5. Synthese der Evaluierung: Darstellung der gesamthaften Wirkung des Programms sowie von Schlussfolgerungen und Anregungen für künftige Forschungsprogramme

6. Reflexion der Evaluierungsergebnisse: Gespräche mit internationalen Experten und Durchführung eines nationalen Reflexionsworkshops mit nationalen Experten 
Folgende methodischen Elemente haben dabei Anwendung gefunden:

* Ziel- und umsetzungsorientierte Nutzung und Auswertung von verfügbaren wissenschaftlichen Studien, Datenmaterialien und Analysen und projektrelevante Darstellung gemäß sozialwissenschaftlicher Normen

* Erstellung von fundierten Kosten-Nutzen-Analysen und Wirkungsanalysen

* Recherche und strukturierte Analyse von Entwicklungen anhand von Primärund Sekundärquellen (zB Zahlen und Daten, strategische Dokumente, Programme)

* Durchführung von mittels Gesprächsleitfaden teilstrukturierten qualitativen (Tiefen-)Interviews sowie Auswertung anhand sozialwissenschaftlicher Standards

* Durchführung von repräsentativen Online-Befragungen sowie Auswertung der Ergebnisse

* Volkswirtschaftliche Analyse der regionalen Wertschöpfungs- und Beschäftigungseffekte

* Durchführung eines Reflexionsworkshops unter Einsatz von interaktiven Workshopmethoden

* Ableitung und Darstellung von umsetzungsorientierten Handlungsansätzen und -empfehlungen 


\section{INPUT: Förderprogramm Haus der Zu- kunft}

Dem o.a. Evaluierungsdesign entsprechend wird der Blick zunächst auf den I NPUT gerichtet, also das Förderprogramm Haus der Zukunft. In zusammenfassender Weise werden die strategischen Rahmenbedinungen, die Ziele und Inhalte des Programms, die operative Umsetzung sowie relevante Umfeldentwicklungen dargestellt.

\subsection{Strategische Einbettung des Programms}

Vor dem Hintergrund der Ölkrise und der Entscheidung der österreichischen Bevölkerung gegen die Atomkraft begann man in Österreich bereits in den 1970er Jahren, sich mit Energieforschung zu befassen. Der Gebäudebereich wurde bereits damals im Rahmen der Bundeswohnbauforschung berücksichtigt. Als die Wohnbauförderung in den 1990er Jahren den Ländern übertragen wurde, wurde auch die Wohnbauforschung vom Bund zu den Ländern übertragen.

Im Lauf der 1980er und 1990er Jahre bildete sich zunehmend ein Bewusstsein für die Bedeutung von Umweltschutz, Energie- und Ressourceneffizienz heraus. Sukzessive wurde auch erkannt, dass in diesem Themenfeld große Chancen für den Wirtschafts- und Forschungsstandort Österreich liegen, der sich nun - nicht zuletzt nach dem EU-Beitritt - durch die Herausbildung von Kompetenzen in Wachstumsfeldern im internationalen Wettbewerb behaupten und profilieren muss.

In den 1990er Jahren wurden in Österreich die ersten Initiativen im Bereich des Passivhausbaus gesetzt - Vorarlberg zeigte sich dabei als großer Vorreiter. Impulsgebend wirkte u.a. die Beteiligung maßgeblicher österreichischer Akteure am EU-Programm CEPHEUS („Cost Efficient Passive Houses as European Standards“), das 1997 begonnen wurde.

Parallel vollzog sich in der nationalen Forschungs- und Innovationsförderung ein Trend zu missionsorientierten F\&E-Programmen. Während der Schwerpunkt davor allein auf der Verbesserung funktionaler und struktureller Bedingungen des Innovationssystems lag (zB Förderung von Forschungseinrichtungen, Unterstützung von Kooperationen zwischen Wissenschaft und Wirtschaft u.dgl.), so wurde nunmehr der Fokus auf zunehmend gesellschaftliche Herausforderungen gelegt, was zur Entwicklung themenspezifischer Programme führte. Erste Forschungsarbeiten befassten sich mit dem Bausektor, im Rahmen von gebäudetechnischen Experimenten wurde bspw. ein „Haus ohne Heizung" erprobt. 
n Verbindung dieser beiden Trends lässt sich ab den 1990er Jahren ein vermehrtes Interesse an Forschung und Innovation im Energie- und Umweltbereich feststellen, welcher sich u.a. in den Schwerpunkten „Energietechnik" und „Umwelttechnik" im damaligen Innovations- und Technologiefonds abbildete. Durch Studien und aktive Netzwerkbildung zur wirtschaftsorientierten Nachhaltigkeitsforschung kristallisierten sich Ende der 1990er Jahre erste Grundzüge und Themenfelder für ein entsprechendes I mpulsprogramm heraus. Hierzu zählten neben Solarenergie, Biomasse, nachwachsenden Rohstoffen, energieeffizienten Prozessen und Verfahren insbesondere auch nachhaltiges Bauen und Wohnen.

Mit diesen Überlegungen waren die inhaltlichen Eckpfeiler des Impulsprogramms „Nachhaltig Wirtschaften“ bereits abgesteckt. Mit dem Start der Programmlinie Haus der Zukunft im Jahr 1999 wurde das Impulsprogramm des BMVIT ausgerollt. Diese war eine von drei Programmlinien.

Durch Erforschung und Entwicklung energie- und umweltorientierter Technologien wollte das Impulsprogramm Innovationen in diesem Zukunftsmarkt initiieren und so zu einer Verbesserung der Lebensqualität im Land und einer Stärkung der (internationalen) Wettbewerbsfähigkeit österreichischer Unternehmen beitragen. Es wurde der Anspruch gestellt, deutlich sichtbare Innovations- und Technologiesprünge zu bewirken, sodass eine hohe Breitenwirkung, Sichtbarkeit und Bewusstseinsbildung mit den Projekten einhergehen sollte. Damit wollten Impulse zur Unterstützung eines Strukturwandels in Richtung nachhaltigen Wirtschaftens gesetzt werden.

Abbildung 2: Ziele des I mpulsprogramms Nachhaltig Wirtschaften

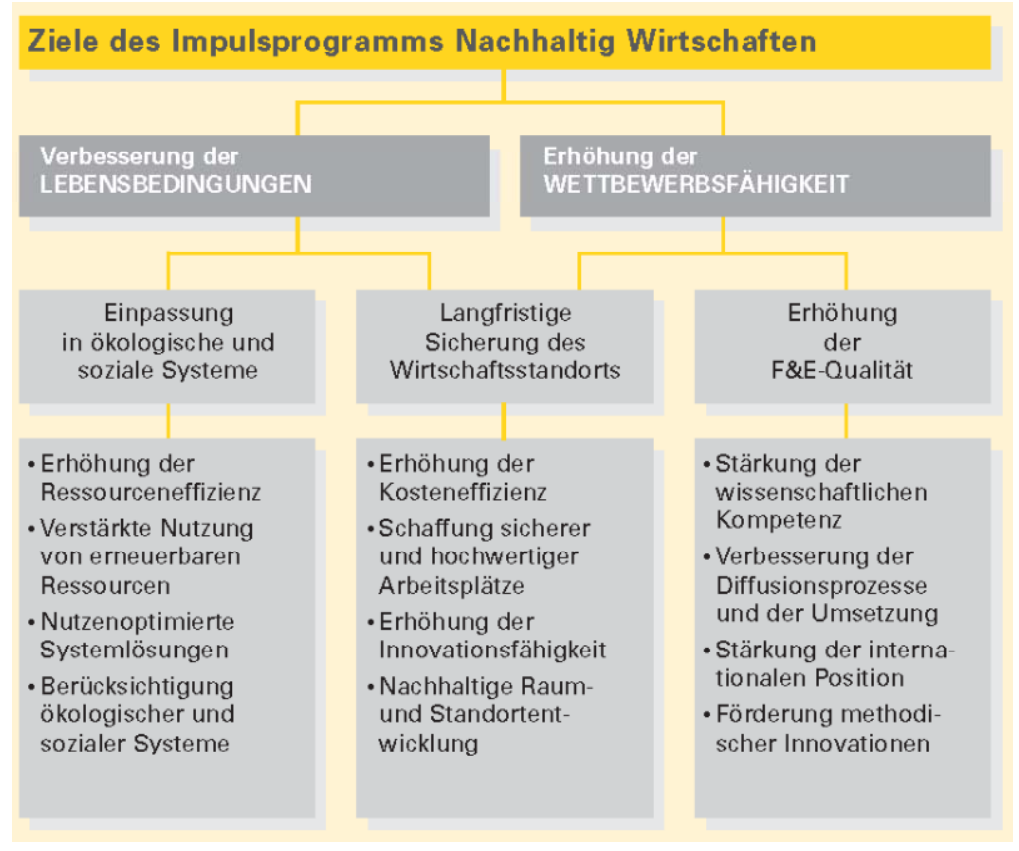

Quelle: BMVIT 2004a, 19 
Im Jahr darauf folgte die zweite Programmlinie „Fabrik der Zukunft“, 2003 schließlich „Energiesysteme der Zukunft“. Für jede Programmlinie wurden klare Zielsetzungen und eine mehrjährige Strategie festgelegt. Auf Basis von Grundlagenstudien, Konzepten sowie Technologie- und Komponentenentwicklung sollten Pilot- und Demonstrationsanlagen umgesetzt werden. Neben der Förderung von Forschungs- und Entwicklungsvorhaben wurden auch Finanzierungsinstrumente als Investitionsanreize und fokussierte Begleitmaßnahmen (Vernetzungsaktivitäten, Qualifizierungsmaßnahmen, Wettbewerbe, Gründungsinitiativen, etc.) gesetzt. 2004 wurde eine Zwischenbilanz des Impulsprogramms „Nachhaltig Wirtschaften“ veröffentlicht.

\section{Abbildung 3: Programmlinien des I mpulsprogramms Nachhaltig Wirtschaften}

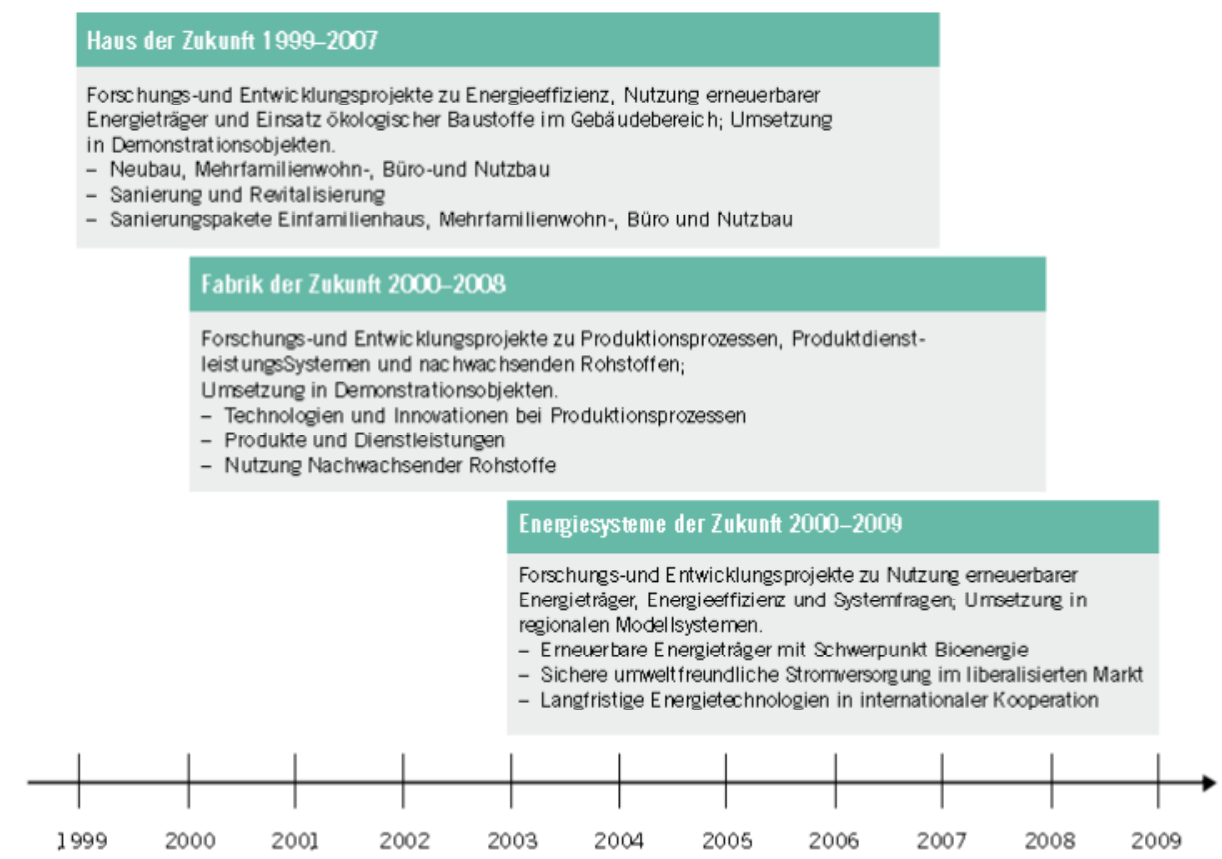

Quelle: FTB 2008, 63

Auf Basis der Ergebnisse des vom BMVIT initiierten und von zahlreichen Experten getragenen Zukunftsprozesses „ENERGI E 2050“ ergaben sich wesentliche Inputs für Haus der Zukunft. Zur Verwirklichung der Ziele (Sicherung des Wirtschaftsstandortes, Erhöhung der F\&E-Qualität, Nachhaltiges Energiesystem, Reduktion der Klimawirkungen) wurden dementsprechend Forschung und Innovation in sechs Themenfeldern forciert: Energiesysteme und Netze, Fortgeschrittene biogene Brennstoffproduktion (Bioraffinierie), Energie in Industrie und Gewerbe, Energie in Gebäuden, Energie und Endverbraucher sowie Fortgeschrittene Verbrennungsund Umwandlungstechnologien. 
Konzepte und Technologien für Nachhaltiges Bauen haben sich in weiterer Folge als zentrales Themenfeld in der Forschungsstrategie des BMVIT etabliert. Dazu wurden insbesondere in der zweiten Programmphase wichtige Impulse gesetzt.

Eine wesentliche Erkenntnis der Forschungs- und Innovationsprojekte im Rahmen von Haus der Zukunft ist die Bedeutung des gesamtheitlichen Zugangs und der Betrachtung von Gebäudeverbünden. Damit war das Programm ein wichtiger Initiator für das 2013 gestartete Nachfolgeprogramm "Stadt der Zukunft", welches auch Strategien, Technologien und Dienstleistungen für ganze Stadtteile oder Städte in den Fokus rückt und nicht nur auf Bautechnologie abzielt, sondern auch auf Energiesysteme, Schnittstelle zu Mobilität, urbane Services uäm.

\subsection{Programmziele}

Die Zielsetzungen des Programms Haus der Zukunft entstanden wie die gesamte Programmlinie aus verschiedenen Vorarbeiten. Die Expertengruppe „R\&D Priority Setting and Evaluation" des Forschungsausschusses der Internationalen Energieagentur ist hierbei ebenso zu erwähnen wie diverse Studien und Pilotprojekte im Rahmen anderer Forschungsförderungsprogramme.

Das Programm wurde in zwei Phasen umgesetzt. Die erste Phase unter dem Titel „Haus der Zukunft" umfasste fünf Ausschreibungen zwischen 1999 und 2005. Ab 2008 wurde das Programm unter dem Titel „Haus der Zukunft plus“ fortgesetzt.

Die Programmlinie Haus der Zukunft baute auf den beiden wichtigsten Entwicklungen im Bereich des solaren und energieeffizienten Bauens auf - der solaren Niedrigenergiebauweise und dem Konzept des Passivhauses. Für Haus der Zukunft wurden diese energieorientierten Innovationen um ökologische, ökonomische und soziale Anforderungen erweitert.

Unter "Haus der Zukunft" waren Neubauten und sanierte Altbauten zu verstehen, die im Vergleich zur konventionellen Bauten folgende Kriterien erfüllten:

* Deutliche Reduzierung des Energie- und Stoffeinsatzes

* Verstärkter Einsatz erneuerbarer Energieträger, insbesondere Solarenergie

* Erhöhte und effiziente Nutzung nachwachsender bzw. ökologischer Materialien

* Berücksichtigung sozialer Aspekte und Erhöhung der Lebensqualität

* Vergleichbare Kosten zur herkömmlichen Bauweise und damit hohes Marktpotenzial 
Ziel von Haus der Zukunft war die Erforschung und Entwicklung von marktfähigen Komponenten, Bauteilen und Baukonzepten für Wohn-, Büround Nutzbauten, die den genannten Kriterien in hohem Maße entsprechen. Zunächst wurde ein Schwerpunkt auf den energieeffizienten Neubau gelegt, der ab der dritten Ausschreibung 2002 um das Thema der Althaussanierung erweitert wurde.

Durch die Förderung von Forschung und Entwicklung sowie die möglichst rasche praktische Erprobung innovativer Lösungen im Rahmen von sichtbaren Demonstrationsprojekten sollte die österreichische Bauwirtschaft im internationalen Wettbewerb gestärkt und eine führende Position in Bezug auf innovative Technologien für Nachhaltiges Bauen erreicht werden. Die grundlegende Erforschung von Technologien sowie deren Marktüberführung waren also bereits zu Beginn des Forschungsprogramms im Fokus.

Im Lauf der Zeit wurden weitere Aspekte des Forschungs- und Innovationsgegenstandes "Nachhaltiges Bauen“ aufgegriffen. Zu Beginn stand die Erforschung wissenschaftlicher und technologischer Grundlagen im Mittelpunkt, worauf hin zunehmend die Entwicklung marktfähiger und kostengünstiger Lösungen unterstützt wurde. Dies geht einher mit der schrittweisen Ausweitung des Programminstruments der Demonstrationsvorhaben, der Fokussierung auf optimierte Gesamtlösungen für Neubau und Sanierung sowie der engen Zusammenarbeit mit der AWS bei Investitionsprojekten.

Die Programmziele und -inhalte wurden kontinuierlich weiterentwickelt, da auf die Erfahrungen und gewonnenen Erkenntnisse aus der ersten Programmphase aufgebaut werden konnte. Im Fokus stand nun die Zielsetzung, dass die technologischen Voraussetzungen für die Herstellung von „Plus-Energie-Gebäuden“ geschaffen werden sollten. Damit sind Gebäude gemeint, die über den gesamten Lebenszyklus mehr Energie erzeugen als sie verbrauchen.

Die zweite Programmphase namens „Haus der Zukunft plus" sollte zur Entwicklung und Vorbereitung bzw. Unterstützung der Markteinführung oder Marktdurchdringung wirtschaftlich umsetzbarer, innovativer technischer und organisatorischer Lösungen im Sinne eines $\mathbf{C O}_{\mathbf{2}}$-neutralen Gebäudesektors einen zentralen Beitrag leisten. Damit sollten die Sicherung zukünftiger Energieversorgung und eine Reduktion der treibhausgasrelevanten Emissionen im Gebäudesektor unterstützt werden. 
Der Zusatz „plus“ in der Bezeichnung der zweiten Programmphase sollte diese Zielsetzung zum Ausdruck bringen und umfasste folgende Stoßrichtungen:

\section{* „Plus“ Technologieführerschaft}

Die durch das Programm „Haus der Zukunft" erreichte Technologieführerschaft in Schlüsseltechnologien des Nachhaltigen Bauens soll ausgebaut werden.

\section{* „Plus“ Energieerzeugung}

Langfristig ist es erforderlich, den Gebäudebereich vom Energieverbraucher zum Energieerzeuger überzuführen. Im Programm Nachhaltig Wirtschaften wurde gezeigt, dass die hierfür erforderlichen Technologien vorhanden sind oder entwickelt und in ein Gesamtkonzept integriert werden können.

\section{* „Plus“ I ndustrielle Umsetzung}

Die Innovationen des Vorläuferprogramms sowie andere innovative Produktentwicklungen sollen durch die Instrumente der aws von der Einzel- zur Serienfertigung übergeführt werden. Dies soll den wirtschaftlichen Erfolg der Unternehmen sicherstellen und damit die Basis für weitere Innovationen stärken.

\section{* „Plus“ Siedlung}

Die Betrachtung von Einzelgebäuden im „Haus der Zukunft“ hat wesentliche technologische Fortschritte initiiert, greift jedoch im Sinne der Herausforderung Klimaschutz zu kurz. Plus steht daher für Siedlungen bzw. Gebäudeverbände und Systemintegration.

\section{* „Plus“ I nternationale Vernetzung}

Österreich wird innerhalb Europas als wesentlicher Innovator im Bereich Energie in Gebäuden wahrgenommen. Diese Position soll durch eine verstärkte internationale Kooperation weiter ausgebaut werden.

\section{* „Plus“ Wissenstransfer und Bildung}

Wesentlich für die Transformation des Bausektors sind der Transfer von Wissen zu Professionisten und die verstärkte Integration der Forschungsergebnisse in die Lehrinhalte.

Auf diese erweiterte Zieldimension wurden die Programminhalte und -instrumente abgestimmt. So wurde etwa mit der ersten Ausschreibung der Programmphase „Haus der Zukunft Plus“ Leitprojekte gefördert. Dabei handelte es sich um integrierte Leuchtturmvorhaben, die aus verschiedenen Teilprojekten bestanden. Weiters wurden neben einzelnen Gebäuden auch Gebäudeverbünde bzw. ganze Siedlungen in den Fokus gerückt. Ein weiterer Schwerpunkt wurde auf die internationale Vernetzung gelegt, da Österreich - nicht zuletzt durch die Projekte im Rahmen von Haus der Zukunft - nunmehr zu den führenden Nationen im Bereich des Nachhaltigen Bauens aufgestiegen war. 


\subsection{Programminhalte}

Das Programm Haus der Zukunft wurde - von Einzelbeauftragungen für Studien, Transfermaßnahmen u.dgl. abgesehen - in erster Linie im Rahmen von Ausschreibungen umgesetzt. Dabei wurden die in der jeweiligen Ausschreibung angestrebten Ziele, Themenfelder und Projektarten explizit adressiert.

Die erste Programmphase "Haus der Zukunft" umfasste fünf Ausschreibungen im Zeitraum 1999-2007. Der Fokus wurde zunächst auf den Neubau im Niedrigenergie- bzw. Passivhausstandard gelegt, ab der dritten Ausschreibung auch auf die Sanierung von Bestandsobjekten. Sukzessive wurden neben Forschungsprojekten und Demonstrationsvorhaben auch Begleitmaßnahmen ausgeschrieben.

In der zweiten Programmphase "Haus der Zukunft plus" wurden von 2008 bis 2012 vier Ausschreibungen vorgenommen, welche im Wesentlichen an der Vision des Plusenergie-Gebäudes ausgerichtet waren. Hierfür wurden bewährte Instrumente durch neue Ansätze ergänzt ( $z B$ Leitprojekte). Die Themenschwerpunkte der Ausschreibungen blieben in ihrer Grundausprägung konstant, fokussierten aber je nach Ausschreibung unterschiedliche Aspekte und Facetten.

Wie in der nachfolgenden Übersicht der zentralen Programminhalte offensichtlich wird, hat sich das Programm im Lauf der Zeit kontinuierlich weiterentwickelt. Dies betrifft sowohl die Projektarten und Instrumente wie auch die Themenschwerpunkte der Ausschreibungen. Zu Beginn standen Studien, Technologieentwicklungen und Baukonzepte im Mittelpunkt, sukzessive wurden auch Demonstrationsvorhaben, Begleitmaßnahmen, Investitionsprojekte, F\&E-Kooperationen uäm. gefördert. In der ersten Ausschreibung der zweiten Programmphase wurden erstmals Leitprojekte gefördert, wofür ein entsprechendes Budget bereitgestellt werden konnte. Demonstrationsprojekte wurden in den meisten Ausschreibungen gefördert - aufgrund der geringen Resonanz der Ausschreibung im Jahr 2009 wurde im Folgejahr die Ausschreibung für Demonstrationsprojekte wiederholt und konkretisiert.

In thematischer Hinsicht waren die ersten beiden Ausschreibungen auf den Neubau fokussiert, im Rahmen der weiteren „Haus der Zukunft“-Ausschreibungen waren Neubau und Althaussanierung die klaren Themenschwerpunkte. In der zweiten Programmphase zieht sich das Ziel des Plusenergie-Gebäudes quasi als roter Faden durch alle Ausschreibungen. Auch die Volumina haben sich - trotz deutlicher Schwankungen - grosso modo gesteigert. 


\begin{tabular}{|c|c|c|c|}
\hline Ausschreibung & Volumen & Projektarten & Themenschwerpunkte (zT Auswahl) \\
\hline $\begin{array}{l}\text { 1. Ausschreibung } \\
\text { Haus der Zukunft } \\
1999\end{array}$ & $€ 3,7$ Mio. & $\begin{array}{l}\text { Grundlagenstudien } \\
\text { Technologie- und } \\
\text { Komponentenent- } \\
\text { wicklung } \\
>\text { Innovative Baukon- } \\
\text { zepte }\end{array}$ & $\begin{array}{l}\text { Grundlagenstudien } \\
-\quad \text { Fördernde und hemmende Faktoren der Markteinführung von in- } \\
\text { novativen Gebäuden } \\
-\quad \text { Untersuchung zur Akzeptanz ökologischer Niedrigenergiebauweise } \\
\text { Technologie- und Komponentenentwicklung } \\
-\quad \text { Teilsolare Raumheizung von Mehrfamilienhäusern } \\
-\quad \text { Effiziente Solartechnologie und deren Integration in die Gebäude- } \\
\quad \text { außenhülle } \\
>\text { Innovative Baukonzepte } \\
-\quad \text { Visionäre Gesamtkonzepte in Mehrfamilien- und Bürobau }\end{array}$ \\
\hline $\begin{array}{l}\text { 2. Ausschreibung } \\
\text { Haus der Zukunft } \\
2000\end{array}$ & $€ 2,8$ Mio. & $\begin{aligned} &> \text { Grundlagenstudien } \\
& \text { Begleitmaßnahmen } \\
&>\text { Technologie- und } \\
& \text { Komponentenent- } \\
& \text { wicklung } \\
&>\text { Innovative Baukon- } \\
& \text { zepte }\end{aligned}$ & $\begin{array}{l}>\text { Grundlagenstudien } \\
-\quad \text { Informationstechnologien im Wohnbau } \\
- \text { Bedürfnisse von Käufern und Bewohnern von Einfamilienhäusern } \\
>\text { Begleitmaßnahmen } \\
-\quad \text { Informationsknoten zur verstärkten Integration ökologischer Mate- } \\
\quad \text { rialien und nachwachsender Rohstoffe } \\
>\text { Technologie- und Komponentenentwicklung } \\
- \text { Solare Raumheizungsanlagen } \\
- \text { Kühlsysteme auf Basis erneuerbarer Energieträger } \\
>\text { Innovative Baukonzepte } \\
- \text { Gesamtkonzepte für den Büro- und Nutzbau } \\
- \text { Gesamtkonzepte für Mehrfamilienwohn- und Burohäuser }\end{array}$ \\
\hline $\begin{array}{l}\text { 3. Ausschreibung } \\
\text { Haus der Zukunft } \\
2002\end{array}$ & $€ 8,3$ Mio. & $\begin{aligned}> & \text { Grundlagenfor- } \\
& \text { schung/-studien } \\
> & \text { Wirtschaftsbezogene }\end{aligned}$ & $\begin{array}{l}\text { Themenschwerpunkt Althaussanierung } \\
- \text { Zukunft der Gebäudesanierung } \\
- \text { Analyse des Sanierungsprozesses }\end{array}$ \\
\hline
\end{tabular}




\begin{tabular}{|c|c|c|c|}
\hline \multirow[t]{2}{*}{ Ausschreibung } & Volumen & Projektarten & Themenschwerpunkte (zT Auswahl) \\
\hline & & $\begin{array}{l}\text { Grundlagenfor- } \\
\text { schung } \\
>\text { Technologie- und } \\
\text { Komponentenent- } \\
\text { wicklung } \\
>\text { I nnovative Baukon- } \\
\text { zepte } \\
>\text { Demonstrationsvor- } \\
\text { haben }\end{array}$ & $\begin{array}{l}\text { - Technologische Innovationen in der Gebäudesanierung } \\
\text { - Sanierungsplanungen } \\
\text { Themenschwerpunkt Neubau } \\
- \text { Innovative Speichertechnologien } \\
- \text { Komponenten und Elemente auf Basis nachwachsender Rohstoffe }\end{array}$ \\
\hline $\begin{array}{l}\text { 4. Ausschreibung } \\
\text { Haus der Zukunft } \\
2003\end{array}$ & $€ 3,1$ Mio. & $\begin{array}{l}\text { Technologie- und } \\
\text { Komponentenent- } \\
\text { wicklung } \\
>\text { I nnovative Sanie- } \\
\text { rungskonzepte im } \\
\text { Wohnbau } \\
\text { Demonstrationsvor- } \\
\text { haben }\end{array}$ & $\begin{array}{l}\text { Themenschwerpunkt Althaussanierung } \\
- \text { Innovative Komponten und Systemlösungen } \\
- \text { Sanierungsplanungen } \\
\text { Themenschwerpunkt Neubau } \\
- \text { Innovative Speichertechnologien } \\
- \text { Technologien zur verstärkten Nutzung erneuerbarer Energieträger }\end{array}$ \\
\hline $\begin{array}{l}\text { 5. Ausschreibung } \\
\text { Haus der Zukunft } \\
2005\end{array}$ & $€ 3,8$ Mio. & $\begin{array}{l}\text { Technologie- und } \\
\text { Komponentenent- } \\
\text { wicklung } \\
\text { Unterstützung von } \\
\text { Demonstrationsvor- } \\
\text { haben } \\
>\text { Begleitmaßnahmen }\end{array}$ & $\begin{array}{l}\text { Technologie- und Kompontenentwicklung } \\
- \text { Entwicklung innovativer Technologien, Systeme, Komponenten und } \\
\text { Bauprodukte } \\
\text { Demonstrationsvorhaben im Bereich Althaussanierung } \\
- \text { Großvolumiger Wohnbau } \\
- \text { Dienstleistungsgebäude und Sonderbauten } \\
- \text { Sanierung von Eigenheimen und Eigenheimsiedlungen } \\
\text { Begleitmaßnahmen } \\
- \text { Aktiver Know-how-Transfer } \\
- \text { Begleituntersuchung von Demonstrationsgebäuden }\end{array}$ \\
\hline
\end{tabular}




\begin{tabular}{|c|c|c|c|}
\hline Ausschreibung & Volumen & Projektarten & Themenschwerpunkte (zT Auswahl) \\
\hline $\begin{array}{l}\text { 1. Ausschreibung } \\
\text { Haus der Zukunft } \\
\text { plus } 2008\end{array}$ & $\begin{array}{l}€ 8 \text { Mio. \& } \\
€ 14,6 \\
\text { Mio. (Leit- } \\
\text { projekte) }\end{array}$ & $\begin{aligned} &> \text { Investive Maßnah- } \\
& \text { men } \\
&> \text { Grundlagenfor- } \\
& \text { schung } \\
&>\text { Technische Durch- } \\
& \text { führbarkeitsstudien } \\
&>\text { Industrielle For- } \\
& \text { schung } \\
&>\text { Experimentelle Ent- } \\
& \text { wicklung } \\
&>\text { Demonstration } \\
&>\text { Vernetzung \& Trans- } \\
& \text { fer }\end{aligned}$ & $\begin{array}{l}\text { Schlüsseltechnologien und Konzepte für Gebäude der Zukunft } \\
-\quad \text { „Auf dem Weg zum Plus-Energie-Haus“ } \\
- \text { Energetische und qualitative Gebäudemodernisierung } \\
>\text { Industrielle Umsetzung innovativer Technologien } \\
>\text { Leitprojekte - auf dem Weg zum Demonstrationsvorhaben } \\
- \text { Gebäude im Verbund - Siedlung } \\
- \text { Plus-Energie-Haus / Plus-Energie-Produktionsbetrieb } \\
- \text { Gebäudemodernisierung } \\
- \text { Energieeffizienz im Low-tech-Gebäude } \\
\text { Strategien, Vernetzung und Ausbildung } \\
- \text { Lebenszyklus-Bewertungskonzepte } \\
-\quad \text { "Low-tech“-Gebäudekonzepte } \\
- \text { Internationale Kooperationsprojekte } \\
- \text { Monitoring und Evaluierung }\end{array}$ \\
\hline $\begin{array}{l}\text { 2. Ausschreibung } \\
\text { Haus der Zukunft } \\
\text { plus } 2009\end{array}$ & $€ 2,6$ Mio. & $\begin{aligned} & \text { Investitionsprojekt } \\
& \text { Grundlagenfor- } \\
& \text { schung } \\
&>\text { Technische Durch- } \\
& \text { führbarkeitsstudie } \\
&>\text { Indust. Forschung } \\
&>\text { Experimentelle Ent- } \\
& \text { wicklung }\end{aligned}$ & $\begin{array}{l}\text { Schlüsseltechnologien und Konzepte für Gebäude der Zukunft } \\
- \text { Low-tech-Gebäude } \\
>\text { Industrielle Umsetzung innovativer Technologien } \\
- \text { Bau- und Dämmstoffe, Fassaden, Wand, Fenster/Türen } \\
- \text { Haustechnik-Systeme } \\
- \text { Gebäudeintegrierte Energie-, Wärme- und Kälteerzeugung } \\
- \text { Energiespeicherung } \\
>\text { Strategien, Vernetzung und Ausbildung }\end{array}$ \\
\hline $\begin{array}{l}\text { 2. Ausschreibung } \\
\text { Haus der Zukunft } \\
\text { plus Demonstra- } \\
\text { tionsprojekte } \\
2010\end{array}$ & $€ 4,6$ Mio. & $\begin{array}{l}\text { Demonstrationspro- } \\
\text { jekte }\end{array}$ & $>$ Plus-Energie-Gebäude mit hoher Signal- und Multiplikationswirkung \\
\hline
\end{tabular}




\begin{tabular}{|c|c|c|c|}
\hline Ausschreibung & Volumen & Projektarten & Themenschwerpunkte (zT Auswahl) \\
\hline $\begin{array}{l}\text { 3. Ausschreibung } \\
\text { Haus der Zukunft } \\
\text { plus } 2011\end{array}$ & $€ 3,5$ Mio. & $\begin{aligned}> & \text { Sondierung } \\
> & \text { Einzelprojekt der } \\
& \text { Industriellen For- } \\
& \text { schung } \\
> & \text { Kooperatives F\&E- } \\
& \text { Projekt } \\
> & \text { F\&E-Dienstleistung }\end{aligned}$ & $\begin{array}{l}\text { Schlüsseltechnologien für Gebäude der Zukunft } \\
-\quad \text { Innovative Fassadenkomponenten und -systeme } \\
- \text { Intelligente Haustechniksysteme } \\
- \text { Gebäudeintegrierte Energieerzeugung und -speicherung } \\
>\text { Industrielle Umsetzung innovativer Technologien } \\
- \text { Überleitung innovativer Technologien und Produkte zur Serien- } \\
\text { bzw. industriellen Fertigung } \\
\text { Demonstrationsgebäude } \\
- \text { Neubauten von Gebäuden und Siedlungen mit Plusenergiestandard } \\
- \text { Sanierungsvorhaben in Richtung Plusenergiestandard } \\
>\text { Strategien, Ausbildung und Vernetzung }\end{array}$ \\
\hline $\begin{array}{l}\text { 4. Ausschreibung } \\
\text { Haus der Zukunft } \\
\text { plus } 2012\end{array}$ & $€ 4,5$ Mio. & $\begin{aligned}> & \text { Sondierung } \\
> & \text { Einzelprojekt der } \\
& \text { Industriellen For- } \\
& \text { schung } \\
> & \text { Kooperatives F\&E- } \\
& \text { Projekt } \\
> & \text { F\&E-Dienstleistung }\end{aligned}$ & $\begin{array}{l}\text { Schlüsseltechnologien für Gebäude der Zukunft } \\
-\quad \text { Innovative Systemlösungen und Technologien zur Gebäudesanie- } \\
\text { rung } \\
- \text { Gebäudeintegrierte Energieerzeugung und - speicherung sowie ge- } \\
\text { bäudeübergreifender Austausch } \\
-\quad \text { Intelligente Haustechniksysteme } \\
-\quad \text { Innovative Gebäudekomponenten und -systeme } \\
>\text { Industrielle Umsetzung innovativer Technologien } \\
-\quad \text { Überleitung innovativer Technologien und Produkte zur Serien- } \\
\quad \text { bzw. industriellen Fertigung } \\
>\text { Demonstrationsgebäude } \\
- \text { Neubauten von Gebäuden mit Plusenergiestandard } \\
- \text { Sanierungsvorhaben in Richtung Plusenergiestandard } \\
>\text { Strategische Arbeiten, Know-how-Transfer und Vernetzung }\end{array}$ \\
\hline
\end{tabular}

Quelle: Eigene Darstellung auf Basis der Ausschreibungsleitfäden 
Weiters waren in den Ausschreibungen die Zielgruppen klar definiert, wobei die Zielgruppen meist je nach Projektart variierten. Entgegen anderer Förderprogramme sind die potenziellen Zielgruppen bei Bauforschungs- und -innovationsprojekten sehr heterogen. Haus der Zukunft richtete sich an universitäre und außeruniversitäre Forschungseinrichtungen, Unternehmen aus Industrie und Gewerbe, spezialisierte Dienstleistungsunternehmen (Archtiktur- und Planungsbüros), Bauträger, Baufirmen uvam. Durch die gezielte Förderung von kooperativen Projekten und teilweise die verpflichtende Bildung von Projektkonsortien, in denen unterschiedliche Einrichtungen vertreten sein mussten, konnte so ein weiterer Impuls zur Vernetzung der Akteure im Baubereich gesetzt werden.

Die adressierten Technologiefelder umfassten zunächst den Neubau, ab der dritten Ausschreibung auch die Sanierung nach Niedrigenergie- bzw. Passivhausstandard. Hierbei gingen stets wissenschaftliche Grundlagenforschung, Komponentenentwicklung, Baukonzepte und konkrete Realisierungen im Rahmen von Demonstrations-, Leit- und Investitionsprojekten miteinander einher, wobei im Lauf der Zeit eine Verlagerung des Schwerpunktes von der Grundlagenforschung und Komponentenentwicklung hin zu ganzheitlichen Lösungen und der Umsetzung in realen Gebäuden festzustellen. Ferner wurde ein zunehmendes Augenmerk auf die Kosten bzw. die Finanzierbarkeit innovativer Lösungen gelegt. Neben technologischen Entwicklungen wurden stets auch die Aufbereitung sozio-ökonomischer Grundlagen berücksichtigt. So beinhaltete etwa die erste Ausschreibung auch Studien über die Akzeptanz von ökologischen Bauformen in der Bevölkerung. Bei den letzten Ausschreibungen wurden im Vorfeld Konsultationsprozesse durchgeführt, sodass Stakeholder und potenzielle Projektträger die Möglichkeit hatten, aktiv Themenvorschläge einzubringen.

Auch die Projektarten haben sich im Lauf der Zeit weiterentwickelt. Als Forschungsprogramm unterstützte Haus der Zukunft die Erstellung wissenschaftlicher Grundlagenstudien in der Regel zu 100 \%, bei der wirtschaftsbezogenen Grundlagenforschung bzw. der Technologie- und Komponentenentwicklung wurde nur ein Teil der Projektkosten gefördert (meist 20 - 50 \%). Ferner wurden besonders innovative Bau- und Sanierungskonzepte in den meisten Ausschreibungen unterstützt. Damit sollte die grundsätzliche Machbarkeit einer Technologieentwicklung bzw. die Zusammenführung unterschiedlicher Projektergebnisse zur Vorbereitung eines Demonstrationsvorhabens untersucht werden. Dieses Instrument erwies sich als besonders hilfreich, den Transfer von der Grundlagenforschung hin zur konkreten Anwendungserprobung zu bewältigen. Eine weitere Besonderheit stellten die Demonstrations- und Leitprojekte dar. Hierbei war der Anspruch, innovative Lösungen entsprechend der Programmziele in die Anwendung zu bringen. Die Förderung orientierte sich dabei an den Mehrkosten, die durch die Innovation entstanden, und nicht den gesamten Baukosten. 
Neben der Vertiefung technologischer Kompetenzen und der Sichtbarmachung der Potenziale Nachhaltigen Bauens in der Praxis war die Stärkung der Wettbewerbskraft der österreichischen Bauwirtschaft durch Forcierung von Forschung und Innovation ein zentrales Ziel von Haus der Zukunft. Dazu leisteten die Forschungsund Demonstrationsprojekte einen wertvollen Beitrag, ergänzend wurden gezielte Begleitmaßnahmen umgesetzt, die sicherlich eine Besonderheit im Vergleich zu anderen Förderprogrammen auf Bundesebene darstellen:

* Informationsveranstaltungen zum Förderprogramm (Vorstellung des Programms in ganz Österreich, Infotour im Vorfeld einer Ausschreibung)

* Beratungsangebote (allgemeine oder vertiefende Beratungsgespräche, „Proposal Checks" in der Antragsphase)

* Vernetzungsworkshops im Rahmen der jeweiligen Ausschreibungen (Vorstellung von Projekten und Ergebnissen, Austausch zwischen den Trägern von Projekten mit ähnlicher thematischer Ausrichtung)

* Themenspezifische Workshops (zB „Haus der Zukunft in der Praxis“)

* Online-Plattform www.hausderzukunft.at (Informationen zum Programm, Projektdatenbank, Projektberichte, Veranstaltungsankündigungen und dokumentationen, aktuelle Informationen zum Thema Nachhaltiges Bauen, Betreuung einer Emailadresse für allgemeine Anfragen etc.)

* Medien- und Öffentlichkeitsarbeit (Newsletter, Presseinformationen, Pressekonferenzen, Poster/Landkarten/Roll-ups u.dgl.)

* Kooperationsveranstaltungen (zB Mitwirkung an Kongressen und Symposien von Partnerinstitutionen)

* Publikationen (zB Beiträge in den Publikationsreihen „Forschungsforum“ und „Energy Innovation Austria“, Broschüren, Berichte)

* Preise/Wettbewerbe (zB „Altbau der Zukunft", „Auszeichnung beachtenswerter Pionierleistungen für den Neubau“)

* Filme/DVDs (U.a. „Bauen mit Hausverstand“, „Highlights aus der Energieforschung", "S-HOUSE - Schiestlhaus - Lust auf Lehm“, „Renovation with Innovation") 


\subsection{Programmumsetzung}

Die Programmumsetzung erfolgte während der gesamten Programmlaufzeit unter der Programmverantwortung und -lenkung des BMVIT, welches das Programm initiiert und finanziert hat. Für die operativen Programmaktivitäten wurden Partnerinstitutionen beauftragt.

Die inhaltliche Programmbegleitung wurden durch die Österreichische Gesellschaft für Umwelt und Technik (ÖGUT) wahrgenommen. Im Rahmen dessen war die ÖGUT in Abstimmung mit dem BMVIT für die inhaltliche Ausrichtung und Weiterentwicklung des Programms, die Betreuung und Vernetzung von potenziellen Einreichern und Projektnehmern, die Einbindung wichtiger Stakeholder uäm. verantwortlich. Weiters hat die ÖGUT Maßnahmen zu Projektgenerierung, Projektcoaching und Dissemination übernommen, zB Vorbereitung von Ausschreibungen, Betreuung von Ausschreibungen, Pre-Proposal Checks, Stellungnahmen zu Zwischen- und Endberichten der Projektnehmer oder Vernetzungsaktivitäten im Inund Ausland.

Die operative Abwicklung der Forschungsprojekte erfolgte zunächst über die Technologieimpulse-Gesellschaft (TiG), später über den Forschungsförderungsfonds. 2004 wurden diese und zwei weitere Einrichtungen zur Forschungsförderungsgesellschaft (FFG) fusioniert, wo Haus der Zukunft im Bereich der Thematischen Programme verankert war. Auf die professionelle Abwicklung der Projekte ist auch zurückzuführen, dass für die vorliegende Evaluierung ab der dritten Ausschreibung von "Haus der Zukunft" eine detaillierte Datenbasis vorliegt.

Die Austria Wirtschaftsservice GmbH (aws) als zentrale Förderbank des Bundes war eng in Haus der Zukunft eingebunden, um die Marktüberleitung der Forschungsergebnisse optimal zu unterstützen. Dies war insbesondere in der zweiten Programmphase relevant, wo eine stärkere Marktorientierung angestrebt wurde. Weiters hat die aws die Administration der Investitionsprojekte abgewickelt und die Koordination der Leitprojekte übernommen.

Diese vier Institutionen - BMVIT, ÖGUT, FFG und aws - bildeten zusammen das Managementteam des Programms, das im Rahmen regelmäßiger Jours fixes den reibungslosen Ablauf der Programmumsetzung sicherstellte.

Die Umsetzung der Begleitmaßnahmen erfolgte durch Ausschreibungen bzw. Beauftragung externer Dienstleister. Vielfach übernahm die ÖGUT im Rahmen der Programmbegleitung auch die Durchführung von Begleitmaßnahmen etwa in den Bereichen Öffentlichkeitsarbeit, Ausrichtung von Workshops, Vorstellung des Programms bei Tagungen und Konferenzen etc. 
Zur Bewertung der Projektanträge wurde ein Bewertungsprozess entworfen, der die Passfähigkeit der Projekte zu den Programmzielen und Ausschreibungsinhalten sicherstellen sollte, um eine entsprechende Priorisierung der Projektanträge vornehmen zu können.

Für die Antragsteller wurde in Leitfäden zu den Ausschreibungen stets das Ziel, die Bewertungskriterien und deren Gewichtung definiert. Im Rahmen der Maßnahmen zur Projektgenerierung wurden auch umfangreiche Beratungsangebote (zB Workshops, Proposal Checks) angeboten.

Die finalen Projektanträge wurden anschließend von den Fachexperten des Managementteams einer Vorbegutachtung unterzogen, wobei zwischen Förderungsund Finanzierungsansuchen zu unterscheiden war. Die Vorauswahl nahm eine Differenzierung zwischen prioritär gereihten, diskussionswürdigen und ausgeschlossenen Projekten vor. Ferner wurde bei manchen Ausschreibungen bzw. Anträgen ein zweistufiges Vergabeverfahren angewendet.

Eine international besetzte Bewertungsjury nahm auf Basis der Vorbegutachtungen die Entscheidung über die Projektförderung vor. Die Besetzung der Jury mit internationalen Experten sollte nicht nur eine Unabhängigkeit in der Entscheidung gewährleisten, sondern auch den Stand der internationalen Forschung in die Bewertung einbeziehen. Im Vorfeld der Jurysitzungen wurden Bewertungshandbücher erstellt, welche den Jurymitgliedern die anzuwendenden Kriterien im Detail erörterten (Kriterien, Indikatoren, Gewichtung anhand eines Punkteschemas).

Die Juryentscheidung wurde den Förderwerbern im Anschluss mitgeteilt und allfällige Auflagen erörtert bzw. zur Offertlegung eingeladen. Abgelehnte Anträge erhielten ein inhaltliches Feedback, das alle negativen und positiven Aspekte des Vorhabens aus Sicht der Jury anführte.

Haus der Zukunft war als lernendes Programm konzipiert. J urysitzungen, Expertenworkshops oder Beratungen für Einreicher wurden bewusst dazu genutzt, Verbesserungsmöglichkeiten zu erkennen und nach Möglichkeit zu berücksichtigen. Die Projektträger wurden mittels einer schriftlichen Befragung um ihr Feedback ersucht. Darüber hinaus wurden Zwischenevaluierungen und der internationale Austausch mit Programmverantwortlichen in anderen Ländern gezielt dazu genutzt, Anregungen für die Programminhalte und - abwicklung aufzugreifen.

In diesem Zusammenhang wurde auch ein Schwerpunkt auf den internationalen Austausch mit anderen Ministerien und Agenturen gelegt. Dieser erfolgte im Rahmen der IEA-Forschungskooperation oder durch bilateralen Austausch, zB mit Vertretern des niederländischen Programms „EnergieSprong“, sowie den Empfang internationaler Delegationen. 


\subsection{Relevante Umfeldentwicklungen}

Forschung und Innovation im Gebäudesektor als Schlüssel zur Erreichung von Klimazielen sind zunehmend in den Fokus gerückt - nicht nur im Rahmen des Programms Haus der Zukunft. Da das Programm in seinen Schwerpunkten, seinen Ergebnissen und Wirkungen nicht losgelöst von diesen Umfeldentwicklungen erfasst werden kann, sondern vielmehr eng damit verbunden ist, werden die relevanten Rahmenbedingungen an dieser Stelle zusammengefasst.

\subsubsection{I nternationale und europäische Ebene}

Auf internationaler Ebene ist die Internationale Energieagentur (IEA) zu erwähnen, welche im Jahr 1974 als Reaktion auf die Ölkrise eingerichtet wurde und eine autonome Einheit der OECD darstellt. Österreich war einer von 16 Gründungsstaaten. Mit der Energieforschung befasst sich das "Committee on Energy Research and Technology", in dem das BMVIT Österreich repräsentiert. Vier Arbeitsgruppen in den Bereichen erneuerbare Energie, Endverbrauchstechnologien, fossile Energie und Fusion begleiten die Arbeit der thematisch zugeordneten Technologieinitiativen und initiieren neue Aktivitäten. Die Republik Österreich ist in den ersten drei Arbeitsgruppen vertreten, österreichische Experten und Unternehmen in zahlreichen Technologieinitiativen und Projekten (sog. „Tasks“ oder "Annexe“).

Im Technologieprogramm IEA-Forschungsskooperation finanziert das BMVIT die österreichischen Teilnahmen an kooperativen Forschungs- und Entwicklungsprojekten. Damit sollen österreichische Kompetenzen verbreitet, Forschungsergebnisse international zugänglich gemacht und der Informationsaustausch mit internationalen Partnerorganisationen vertieft werden. Im Zeitraum 2005-2010 wurden 73 Projekte, v.a. in den Themenfeldern Bioenergie und Solarthermie, finanziert. 2015 standen dafür über $€ 2$ Mio. zur Verfügung, die Abwicklung erfolgte über die FFG.

Die Europäische Union wirkte und wirkt in mehrfacher Weise stimulierend auf die österreichischen Aktivitäten: Zum Einen werden auf europäischer Ebene Richtlinien und Standards gesetzt, zum Anderen setzt die EU im Forschungsbereich durch Förderprogramme, Plattformen und Initiativen wichtige Impulse.

Aktuell rückt die Strategie „Europa 2020“ nachhaltiges Wachstum als eines von drei Leitzielen in den Fokus der gesamten europäischen Politik. Eine eigene Leitinitiative „Ressourcenschonendes Europa“ konkretisiert die Überlegungen der Kommission im Energie- und Umweltbereich, wobei der Gebäudesektor ein wichtiger Schlüssel zur Erreichung der so genannten „20-20-20-Ziele“ ist, welche bereits 2009 im Klima- und Energiepaket festgeschrieben wurden: Bis 2020 sollen $20 \%$ weniger Treibhausgasemissionen als 2005, $20 \%$ Anteil an erneuerbaren Energien und $20 \%$ mehr Energieeffizienz erzielt werden. 
2010 wurde die EU-Richtlinie zur Energieeffizienz von Gebäuden von 2002 umfassend abgeändert. Diese Richtlinie liefert die Basis für die Bewertung der Energieeffizienz von Gebäuden, und bezieht sich auf Energieausweise, die für neue Gebäude sowie Gebäude, die einer umfangreichen Sanierung unterzogen werden, nunmehr ausgestellt werden müssen. Diese Richtlinie bildet den Rahmen für die nationale Gesetzgebung zur Energieeinsparung bei Gebäuden. Mit der Neufassung der Energieeffizienz-Richtlinie für Gebäude wurden einige bis dahin bestehende Einschränkungen (zB zum Geltungsbereich) beseitigt. Weiters wurde festgelegt, dass bis zum Jahr 2020 alle Neubauten das Anforderungsniveau von nahezu Nullenergiehäusern erfüllen müssen. Das „nearly zero-energy building“ wird damit europaweit zum Standard erklärt. Für öffentliche Gebäude muss diese Vorgabe bereits 2018 erreicht werden. Die Mitgliedstaaten haben daher entsprechende Maßnahmen im Gebäudebereich umzusetzen.

Zur Erreichung der europäischen Klimaziele ist der Strategieplan für Energietechnologie (SET-Plan) ein wesentliches Instrument, wobei der Schwerpunkt auf der Entwicklung $\mathrm{CO}_{2}$-armer Energietechnologien liegt. Dazu werden einerseits Europäische Industrie-I nitiativen eingerichtet - dabei handelt es sich um groß angelegte Technologieentwicklungsprojekte in Kooperation von Wissenschaft, Forschung und Industrie. Andererseits haben sich unter dem strategischen Dach des SET-Plans 15 führende Energieforschungsinstitutionen in einem europäischen Energieforschungsbündnis zusammengeschlossen, um europäische und nationale Ressourcen und Synergien bestmöglich nutzen zu können. Österreich ist dabei durch das Austrian Institute of Technology (AIT) in den Joint Programmes "Photovoltaik", "Smart Cities" (Programmkoordination AIT) und "Smart Grids" vertreten. Deutlich wird hieraus bereits die auf europäischer Ebene anerkannte Kompetenz Österreichs im Bereich der nachhaltigen Bauforschung.

Der SET-Plan wird laufend weiterentwickelt. Ein Konzept der Europäischen Kommission vom Herbst 2015 („Accelerating the European Energy System Transformation") führt dazu einige zentrale Überlegungen an. Energieeffiziente Gebäude spielen dabei eine immer wichtigere Rolle, insbesondere in Hinblick auf energieeffiziente Heiz- und Kühllösungen, die Entwicklung und Verbreitung neuer Materialien und Technologien für energieeffiziente Gebäude sowie die Kostensenkung innovativer Lösungen durch einen industrialisierten Bauprozess. Auch werden erneut die Bedeutung von Forschung und Entwicklung und die großen Marktchancen europäischer Unternehmen im Bereich des Nachhaltigen Bauens unterstrichen.

Unlängst hat die Europäische Politik ihrem Bestreben zur Sicherstellung von sicherer, bezahlbarer und klimafreundlicher Energie durch die die Veröffentlichung einer „Rahmenstrategie für eine europäische Energieunion“ Nachdruck verliehen. Das erste im Sommer 2015 vorgestellte Maßnahmenpaket spricht dabei u.a. nachhaltige Gebäude an. Im Verkehrs- und Gebäudesektor sieht die Kommission in 
Europa noch große Energieeffizienzpotenziale, weshalb sie Forschung und Innovation in diesem Themenfeld in den kommenden Jahren weiter forcieren möchte. Die konkreten Umsetzungsmechanismen zur Energieunion sind derzeit noch in Verhandlung.

Wie eingangs erwähnt definiert die Europäische Union nicht nur mittel- und langfristige politische Strategien, sondern setzt auch durch konkrete Förderprogramme und Initiativen konkrete Schritte. Das aktuell wichtigste Forschungsprogramm der Union - Horizon 2020 - mit einem Volumen von $€ 80$ Mrd. für die Jahre 20142020 ist dabei an erster Stelle zu erwähnen. Horizon 2020 ist anhand von drei Säulen aufgebaut: „Wissenschaftsexzellenz", „Führende Rolle der Industrie“ und "Gesellschaftliche Herausforderungen".

Im Rahmen der zweiten Säule wird ein Schwerpunkt auf grundlegende und industrielle Technologien (Leadership in enabling and industrial technologies, LEIT) gelegt, wobei Grundlagenforschung und angewandte Forschung verfolgt werden. Das aktuelle Arbeitsprogramm für die J ahre 2016-2017 sieht hierbei einen Schwerpunkt auf energieeffiziente Gebäude vor. Konkret sind folgende Ausschreibungen mit einem Volumen von über $€ 100$ Mio. geplant:

* Highly efficient insulation materials with improved properties

* Performance indicators and monitoring techniques for energy-efficiency and environmental quality at building and district level

* Integration of advanced technologies for heating and cooling at building and district level

* New technologies and strategies for the development of pre-fabricated elements through the reuse and recycling of construction materials and structures

* Development of nearly zero energy building renovation

* Highly efficient hybrid storage solutions for power and heat in residential buildings and district areas, balancing the supply and demand conditions

* Integration of energy harvesting at building and district level

* New business models for energy-efficient buildings through adaptable refurbishment solutions

Darüber hinaus widmet sich Horizon 2020 in der dritten Säule zentralen gesellschaftlichen Herausforderungen. Hierunter sind die Themenfelder "Energie" mit einem Gesamtvolumen von $€ 6$ Mrd. und "Klimaschutz/Rohstoffe" mit $€ 3$ Mrd. von grundsätzlicher Relevanz für das Programm Haus der Zukunft, jedoch von einer sehr großen inhaltlichen Bandbreite gekennzeichnet. Zur Herausforderung der Energieversorgung sind für die Jahre 2016-2017 Ausschreibungen zu For- 
schungs- und Innovationsprojekten im Gebäudebereich geplant, die mit insgesamt $€ 194$ Mio. gefördert werden:

* Supporting accelerated and cost-effective deep renovation of buildings through Public Private Partnership (EeB PPP)

* Overcoming market barriers and promoting deep renovation of buildings

* Integration of Demand Response in Energy Management Systems while ensuring interoperability through Public Private Partnership (EeB PPP)

* Cost reduction of new Nearly Zero-Energy buildings

* Construction skills

Bereits 2008 wurde die „Public-Private-Partnership on Energy-efficient Buildings" lanciert, welche nun unter Horizon 2020 ihre Fortsetzung findet. Im Mittelpunkt dieser Initiative stehen leistbare, bahnbrechende neue Technologien und innovative Lösungen für nachhaltige Gebäude und die "Smart City“. Die Plattform ist als Kooperation zwischen der EU-Kommission und der „Energy Efficient Buildings Association“ institutionalisiert und verfügt über ein Budget von $€ 600$ Mio. für die laufende Finanzperiode.

Die Themenschwerpunkte für die Forschungs- und Innovationsaktivitäten der Public-Private-Partnership sind in einer „Multiannual Roadmap“ festgehalten. Diese sieht vor, bis zum Jahr 2020 nicht weniger als eine Milliarde Euro für Forschungsund Innovationsprojekte in folgenden Bereichen zu lukrieren:

* Design: Integrierte und ganzheitliche Planung eines Gebäudes unter Berücksichtigung aller Komponenten sowie des lokalen räumlichen Umfeldes („multiscale approach“, Unterstützung der Planung für leistbare Nachhaltigkeit, Entwicklung von Tools für die Erfassung und Berücksichtigung von Lebenszyklusinformationen

* Gebäudestruktur: Wiederverwendung von bestehenden Strukturen unter energetischen Gesichtspunkten, leistbare und passfähige Bausysteme mit geringem $\mathrm{CO}_{2}$-Gehalt (inkl. "grauer Energie“)

* Gebäudehülle: Energieeffiziente Dach- und Fassadensysteme, Verbesserung in den Bereichen Ästhetik, Akustik und Lichtnutzung, adaptive Systeme der Außenhülle, Nutzung einfallender Solarenergie

Energietechnik: Energiesparende und „intelligente“ Energielösungen, zB in den Bereichen Heizung, Kühlung, Beleuchtung; Entwicklung europäischer Standards für Energieperformance und Energieauszeichnung, Forcierung und Weiterentwicklung von Building Energy Management Systems (BEMS) etc.

* Bauprozess: Sichere und kostengünstige Lösungen für energieeffizientes Bauen und qualitätsorientiertes Baumanagement, Nutzung neuer IKT- 
Anwendungen, Entwicklung von industriell vorgefertigten Komponenten, Maßnahmen zur Kompetenzsteigerung etc.

* Betriebsmonitoring: Entwicklung eines europäischen Rahmens für die Datenmessung und -analyse, Wartungstechniken zur Energieoptimierung von Gebäuden, Einrichtung einer europaweiten Datenbank

* Lebensende: Innovative Lösungen zur Nachnutzung von Gebäudekomponenten und Baumaterialien

* Querschnittsthemen: Supply-Chains für energieoptimierende Sanierungen, Berücksichtigung der Gebäudenutzung und Nutzerintegration, systemische Integration von Komponenten und Sub-Systemen etc.

Abseits von Horizon 2020 setzt die EU gezielte Maßnahmen in der Forschungs- und Technologiepolitik. Ein wichtiges Instrument sind die Netzwerke im Rahmen des Europäischen Forschungsraumes. Ziel dieser ERA-NETs ist die Förderung und Kooperation zwischen nationalen und regionalen Forschungsförderorganisationen bzw. Programmagenturen, um durch die bessere Abstimmung und Synchronisation nationaler Programme zu einer Überwindung der Fragmentierung des Europäischen Forschungsraumes und einer besseren Nutzung der Ressourcen für FTI beizutragen. Vier ERA-NETs weisen eine inhaltliche Relevanz für das Themenfeld des Nachhaltigen Bauens auf und stehen österreichischen Akteuren offen:

* M-ERA.NET: M-ERA.NET ist ein europäisches Netzwerk zur Koordination von nationalen und regionalen Forschungsprogrammen im Bereich „Materials Science and Engineering". Ziel ist es, transnationale Partnerschaften von Forschungseinrichtungen und Unternehmen in allen Wissens- und Anwendungsgebieten moderner Materialwissenschaft zu unterstützen.

* SOLAR-ERA.NET: SOLAR-ERA.NET ist ein Netzwerk von mehr als 20 Forschungs- und Innovationsprogrammen im Bereich der Solarenergietechnik im Europäischen Forschungsaum. Die Aktivitäten sind eng mit der Solar Europe Industry Initiative (SEII) verbunden. Österreichischen Partnern steht für Projekte im Rahmen von SOLAR-ERA.NET ein Budget von $€ 0,5$ Mio. zur Verfügung.

* ERA-NET Smart Grids Plus: Das ERA-Net Smart Grids Plus ist bestrebt, die Integration von "Smart Grid"-Systemen und -Technologien und deren Marktumsetzung in Europa voranzutreiben. Cross-sektorale und interdisziplinäre Systeminnovationen sind dabei von besonderem Interesse.

* ERA-NET Cofund Smart Cities and Communities: Ziel des ERA-NET Cofund "Smart Cities and Communities" (ENSCC), in dem 12 europäische Staaten zusammenarbeiten, ist die Umsetzung transnationaler F\&E-Projekte mit dem Fokus auf Demonstration und Implementierung von "Smart City"-Ansätzen in den Bereichen Energiesystem, Logistik, Big Data und "Smart Governance“. 
Ein weiteres zentrales Instrument der europäischen Forschungspolitik sind die Initiativen zur gemeinsamen Programmplanung (Joint Programming Initiativen, JPIs). Auch sie sind an den zentralen gesellschaftlichen Herausforderungen ausgerichtet und zielen auf eine bessere Koordination nationaler Forschungspolitiken ab. Im Rahmen der einzelnen JPIs sollen gemeinsame Forschungsagenden der teilnehmenden Mitgliedstaaten erarbeitet werden, um in weiterer Folge die strategische Zusammenarbeit zwischen bereits existierenden nationalen Programmen auszubauen und neue Förderprogramme gemeinsam ein- bzw. auszurichten. Derzeit bestehen zehn JPIs.

Das BMVIT war 2010 maßgeblich in die Initiierung der JPI „Urban Europe Global Urban Challenges, J oint European Solutions“ involviert. Dabei handelt es sich um ein transnationales Forschungsprogramm unter der Schirmherrschaft des europäischen Ministerrates, in dem grundlegenede systemrelevante Fragestellungen zur urbanen Entwicklung behandelt werden.

In thematisch ausgerichteten Europäischen Technologieplattformen (ETPs) stimmen sich Akteure von industrieller und öffentlicher Seite ab und entwickeln gemeinsame Forschungsagenden. Sie stellen kein Förderinstrument dar, beraten allerdings die Europäische Kommission in der Gestaltung ihrer Forschungspolitik, wie etwa bei der Erstellung der Horizon 2020-Arbeitsprogramme und beeinflussen so deren Themen und Umsetzung maßgeblich mit. Derzeit bestehen knapp 40 ETPs. Generell unterscheiden sich die ETPs nicht nur hinsichtlich ihrer Themen, sondern zum Teil auch beträchtlich in ihrer Struktur und Größe voneinander. Relevanz für das Themenfeld Nachhaltiges Bauen weisen die folgenden ETPs auf:

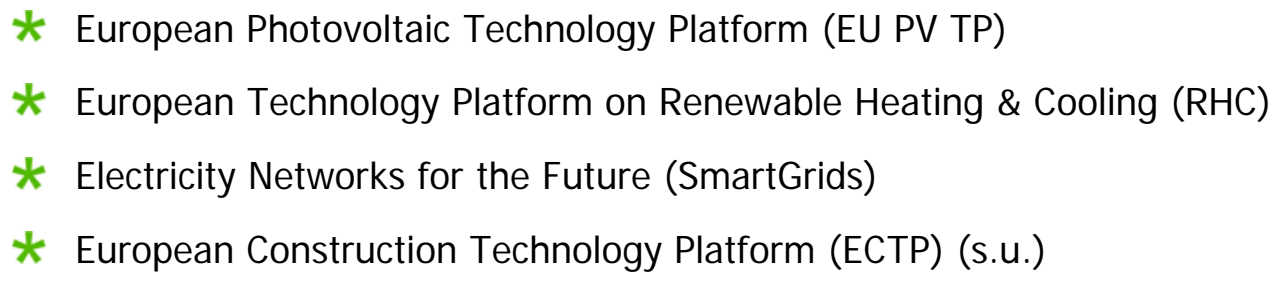

Die „European Construction Technology Platform" hat sich zum Ziel gesetzt, den Stellenwert, die Performance und Wettbewerbskraft der europäischen Bauwirtschaft zu stärken. Hierzu werden die zentralen gesellschaftlichen und technologischen Entwicklungen analysiert und entsprechende Forschungs- und Innovationsstrategien erarbeitet. Die Vernetzung von Akteuren aus unterschiedlichen Bereichen und weiten Teilen Europas sowie die Langfristigkeit der Perspektive sind dabei wichtige Anliegen. Von den derzeit 168 beteiligten Organisationen kommen drei aus Österreich (AIT, ÖGUT, Österreichische Bautechnik Vereinigung). 


\subsubsection{Bundesebene}

Die Chancen und Potenziale Nachhaltigen Bauens in Hinblick auf die Erreichung von Klimazielen sowie die Bedeutung von Forschung und Innovation in diesem Sektor haben in Österreich in den letzten Jahren massiv zugenommen. Dies drückt sich in entsprechenden Strategien und Aktionsplänen des Bundes ebenso aus wie in konkreten Programmen und Initiativen, die durch den Bund eingerichtet wurden.

Bereits seit den 1980er Jahren sind in Österreich Bestrebungen feststellbar, den Energieeinsatz im Gebäudesektor zu reduzieren. Ab den 2000er Jahren haben Energieeffizienz und erneuerbare Energien massiv an Bedeutung in bundespolitischen Strategien und Programmen gewonnen. Zu erwähnen sind hierbei etwa die folgenden Strategien:

* Nationale Klimastrategie (2002 bzw. 2007)

* Energieeffizienz-Aktionsplan (2007)

* Energiestrategie Österreich (2010)

* Nationaler Aktionsplan für erneuerbare Energien (2010)

* Bundesklimaschutzgesetz (2011)

* Österreichische Strategie zur Nachhaltigen Entwicklung (2012)

* Energieeffizienzgesetz (2013)

Auch in den letzten Regierungsprogrammen fand die Bedeutung des Nachhaltigen Bauens im Zusammenhang mit den nationalen Bemühungen zur Erreichung der Klimaziele in vielfacher Weise Eingang.

Die österreichische Forschungspolitik hat - ausgehend von Haus der Zukunft vermehrt die Potenziale dieses Themenfeldes aufgegriffen. Die FTI-Strategie des Bundes sieht - dem "challenge-based approach" folgend - Forschung und Innovation als wichtigen Schlüssel zur Bewältigung des Klimawandels. Explizit werden die Bereich Gebäude, Verkehr und Produktion angeführt, in denen weitere Forschungen zur Steigerung der Energieeffizienz erforderlich sind.

Neben der Möglichkeit, in themenoffenen Programmen wie den FFGBasisprogrammen entsprechende Projekte einzureichen, hat die österreichische Forschungspolitik in den letzen Jahren einen deutlich sichtbaren Schwerpunkt auf die Energieforschung gelegt - „Energie der Zukunft“, „e!mission“, „Energieforschung 2020" sind Beispiele dafür. Das Impulsprogramm "Nachhaltig Wirtschaften“ mit der ersten Programmlinie Haus der Zukunft hat hierfür den Anstoß gegeben. 
Im Rahmen des Strategieprozesses e2050 wurde vom BMVIT und dem Rat für Forschung und Technologieentwicklung unter Einbindung zahlreicher Experten aus Industrie und Forschungseinrichtungen ein Expertenpapier „Energieforschungsstrategie für Österreich“ erarbeitet. Es sieht eine inhaltliche Fokussierung der österreichischen Energieforschung auf Energieeffizienz, erneuerbare Energieträger und intelligenten Energiesysteme vor. Unter den prioritären Themenfeldern ist auch der Gebäudesektor genannt:

* Energiesysteme und Netze

* Fortgeschrittene biogene Brennstoffproduktion (inkl. Bioraffinerie)

* Energie in Industrie und Gewerbe

* Energie in Gebäuden

* Energie und Endverbraucher

* Fortgeschrittene Verbrennungs- und Umwandlungstechnologien

Das BMVIT-Programm „Energieforschung 2020“ strebt an, eine radikale Umgestaltung des Energiesystems zu unterstützen. Dies ist erforderlich, um die internationalen und nationalen Klimaschutzziele zu erreichen, wonach die Industriestaaten ihre Emissionen und ihren Energieverbrauch massiv absenken sollen. Die Energieforschung soll der Komplexität der Thematik gerecht werden und nicht nur neue Technologien und Lösungen hervorbringen, sondern diese auch zu intelligenten Gesamtsystemen mit hoher Akzeptanz der Zielgruppen verbinden. Dementsprechend sind die Ziele der österreichischen Energieforschung definiert:

* Nachhaltiges Energiesystem: Gezielte Impulse zur Reduktion des Energieverbrauchs und Nutzung erneuerbarer Energieträger

* Reduktion der Klimawirkung: Minderung der Treibhausgaseffekte durch Effizienz, Erneuerbare Energieträger und Energiesysteme

* Erhöhung der Wettbewerbsfähigkeit: Ausbau der Führungsrolle im internationalen Wettbewerb

* Erhöhung der F\&E-Qualität: Ausbau der Exzellenz

Derzeit liegen die Schwerpunkte der vom BMVIT forcierten Energieforschung auf Stadt- und Gebäudetechnologien, Smart Grids und Smart Cities.

2004 hat das Lebensministerium die österreichische Klimaschutzinitiative klima:aktiv ins Leben gerufen. Zentrales Anliegen ist die nationale Umsetzung der Ziele der Klimarahmenkonvention der Vereinten Nationen („Kyoto-Protokoll“) durch diverse Programme in unterschiedlichen Themenfeldern. Das klima:aktivProgramm „Bauen und Sanieren“ basierte maßgeblich auf dem im Rahmen von Haus der Zukunft gewonnenen Know-hows. 
in zentrales Element des Programms ist der klima:aktiv Gebäudestandard ${ }^{2}$, welcher der Zertifizierung von Neubauten und Sanierungen von Wohn- und Dienstleistungsgebäuden dient und damit ein wichtiges Leitinstrument des österreichischen Bausektors darstellt. Ferner ist der klima:aktiv Gebäudestandard in zahlreichen Regierungsdokumenten und vielfach in Landesrecht (Wohnbauförderung) manifestiert. Für die Entwicklung dieses Standards war das „Total Quality-Konzept“ des Österreichischen Ökologie-Instituts maßgeblich, welches im Rahmen eines Haus der Zukunft-Projekts entwickelt wurde.

Im Jahr 2007 hat die Bundesregierung mit dem Klima- und Energiefonds (KLIEN) eine eigene klimaorientierte Fördereinrichtung geschaffen. Ziel des Fonds ist die Umsetzung einer nachhaltigen Energieversorgung, die Reduktion der Treibhausgas-Emissionen sowie die Steigerung der Forschungsquote. Gefödert werden Forschungsprojekte im Bereich nachhaltiger Energietechnologien und der Klimaforschung, Projekte des öffentlichen Personennah- und Regionalverkehrs, des umweltfreundlichen Güterverkehrs sowie des Mobilitätsmanagements und die Unterstützung der Markteinführung und Marktdurchdringung von klimarelevanten und nachhaltigen Energietechnologien.

Die Entwicklung der Energieforschung in Österreich lässt sich aus der jährlich nach IEA-Systematik erstellten Energieforschungserhebung nachvollziehen, die von der Austrian Energy Agency erstellt wird. Wie die nachfolgende Abbildung zeigt, hat die Energieforschung in Österreich in den letzten Jahren einen immer höheren Stellenwert eingenommen. Im Jahr 2014 wurden insgesamt $€$ 143,1 Mio. für Energieforschung in Österreich ausgegeben, was einen historischen Höchststand markiert.

Rund $4 \%$ der Bruttoinlandsausgaben für Forschung und Entwicklung entfallen auf die Energieforschung. Damit hat sich der Anteil der Energieforschungsausgaben an den gesamten Forschungsausgaben seit Ende der 1990er Jahre fast verdoppelt. Obgleich die Energieforschung relativ starken Schwankungen unterliegt - dies ist vermutlich auf die Bedeutung großvolumiger Ausschreibungen in den relevanten Programmen zurückzuführen -, so ist doch der Trend deutlich erkennbar. Ab dem Jahr 2012 ist ein leichter Rückgang bei den Energieforschungsausgaben festzustellen, während die gesamten Forschungsausgaben kontinuierlich gestiegen sind.

2

Der klima:aktiv-Gebäudestandard berücksichtigt neben der Energieeffizienz des Gebäudes auch die Planungsund Ausführungsqualität, die Qualität der Baustoffe und Konstruktion sowie zentrale Aspekte zu Komfort und Raumluftqualität. Die Bewertung erfolgt anhand eines Katalogs unterschiedlich gewichteter Kriterien in einem Punktesystem mit max. 1.000 Punkten. Dabei können drei Qualitätsstufen erreicht werden: Bronze (bei Erfüllung der Pflichtkriterien), Silber (mind. 750 Punkte) und Gold (mind. 
Programmevaluierung Haus der Zukunft 1999 - 2013

Abbildung 4: Anteil der Energieforschung an den Bruttoinlandsausgaben (Bund und Länder) für F\&E
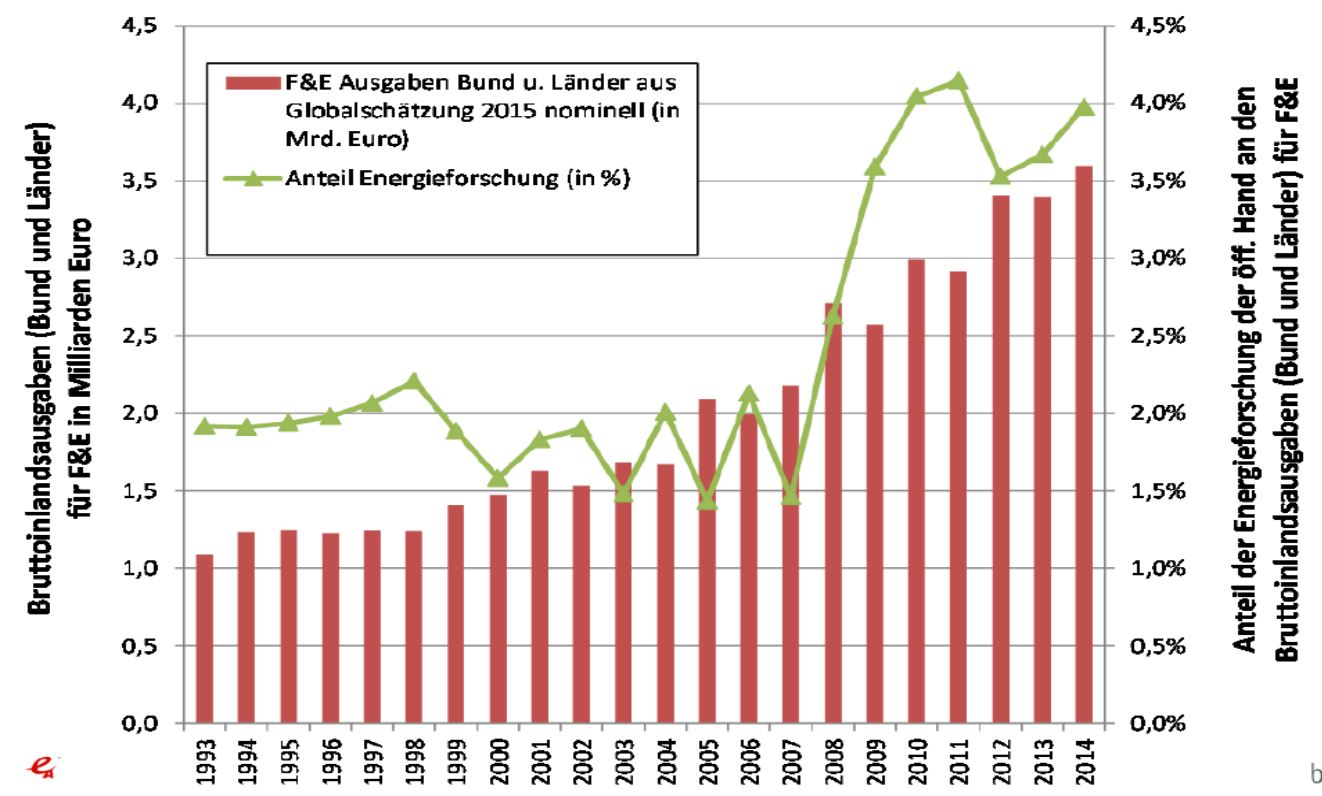

Quelle: AEA, Energieforschungserhebung 2014

Die IEA-Systematik unterscheidet die Energieforschung nach bestimmten Sektoren. Im Sektor „Gebäude und Geräte“ zeigt sich ein deutlicher Anstieg ab dem Jahr 2008. € 18,2 Mio. wurden im Jahr 2014 für Forschungsprojekte und - institutionen in diesem Themenfeld aufgebracht.

Abbildung 5: Energieforschungsausgaben im Themenfeld „Gebäude und Geräte“ von allen Einrichtungen 2005 - 2014

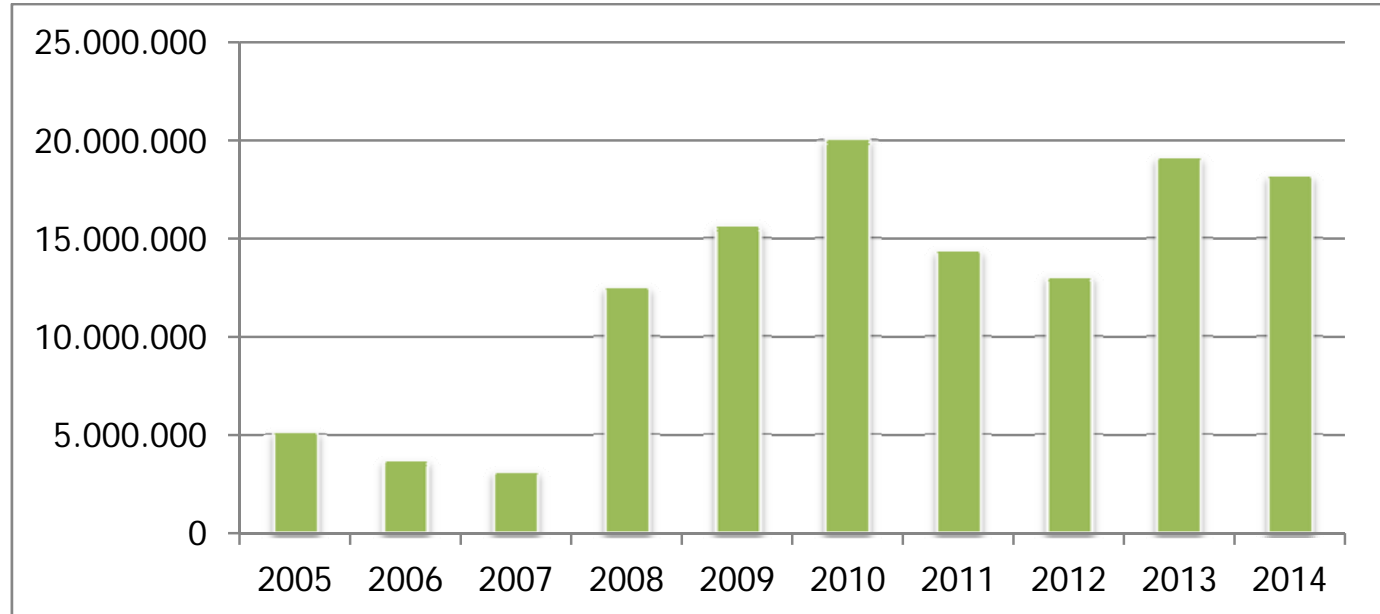

Quelle: Energieforschungserhebungen 2005-2014 
nnerhalb dieses breiten Segments lassen sich die gebäuderelevanten Energieforschungsausgaben extrahieren. Hierzu wurden die relevanten Subkategorien der Erhebung ${ }^{3}$, welche sich im Betrachtungszeitraum leicht verändert haben, kumuliert. Auch hier ist eine deutliche Zunahme ab 2008 festzustellen. Knapp $€ 14$ Mio. wurden im Jahr 2014 für gebäuderelevante Energieforschung in Österreich aufgewendet.

Abbildung 6: Gebäuderelevante Energieforschungsausgaben von allen Einrichtungen $2005-2014$

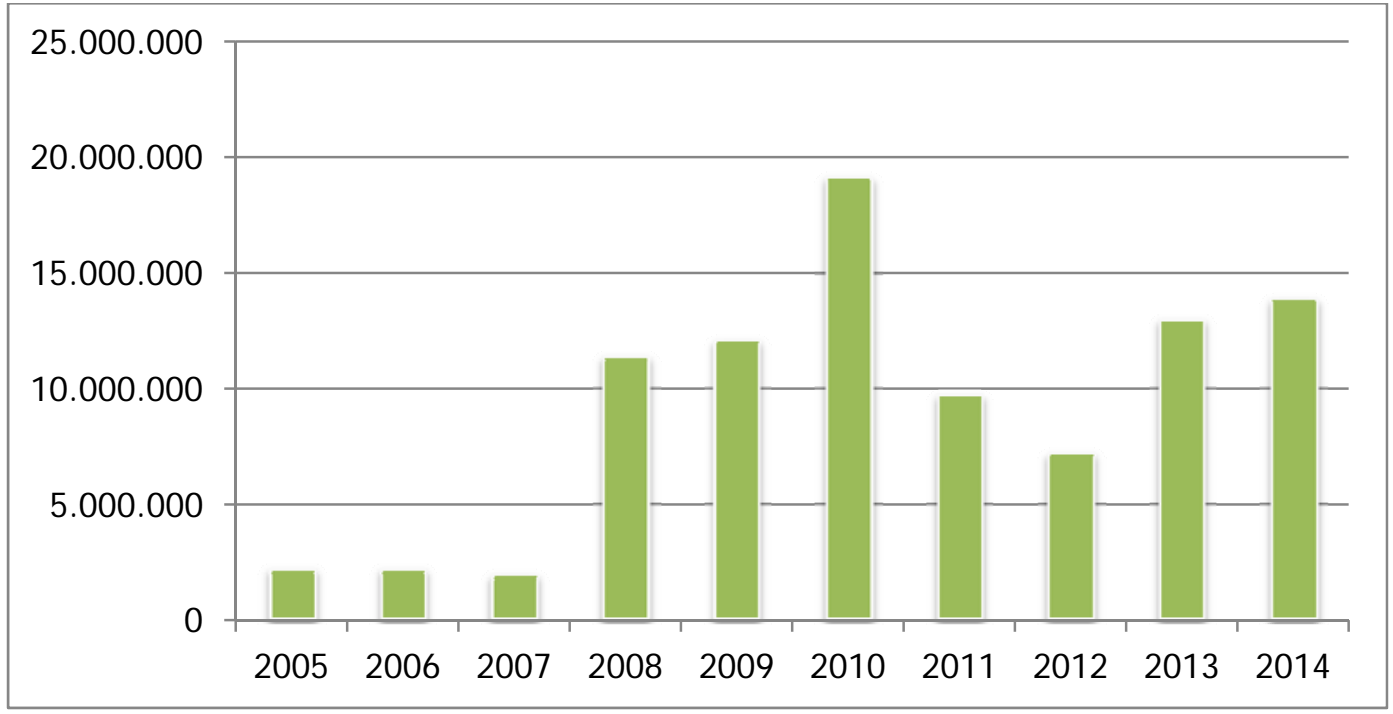

Quelle: Energieforschungserhebungen 2005-2014; eigene Berechnungen

\subsubsection{Länderebene}

Forschung und Innovation im Energie- und Umweltbereich im Allgemeinen und im Themenfeld des Nachhaltigen Bauens im Besonderen hat in den letzten Jahren auch auf Ebene der österreichischen Bundesländer an Stellenwert gewonnen. Dies ist zum Großteil darauf zurückzuführen, dass die über 30 Jahre hinweg beim Wirtschaftsministerium angesiedelte Wohnbauforschung 1988 auf die Bundesländer übertragen wurde. Dies erfolgte im Zuge mit der generellen Übertragung der Wohnbauförderungskompetenzen vom Bund an die Länder - zugleich wurde die Zweckbindung aufgehoben, was zu einer Reduzierung der entsprechenden Fördermittel führte. Ferner beinhaltete die Wohnbauforschung ein breites Themenspektrum, etwa auch Fragen des Wohnraumbedarfs, der Finanzierung etc.

3 Subkategorien: Heizung, Kühlung, Klimatisierung und Beleuchtung; Design für Niedrigenergie- und Passivhäuser; Neue Materialien (inkl. Dämmung); Thermisches Verhalten von Gebäuden; Technologien der Gebäudehülle; Planung und Design; Energiemanagementsysteme für Gebäude und effiziente Internet- und Kommunikationstechnologien; Beleuchtung inkl. Kontrollsysteme; Gebäudetechnik und Betrieb 
Mit Fokus auf die Forschung im Bereich Nachhaltiges Bauen ist festzuhalten, dass die Bundesländer in ihren eigenen Forschungsbudgets der Energieforschung zunehmend Priorität einräumen. Laut Energieforschungserhebung haben die Länder im Jahr 2014 insgesamt $€ 11,4$ Mio. in die Energieforschung investiert. Dieser Wert markiert einen historischen Höchststand, wenngleich die Energieforschungsausgaben der Länder deutlichen Schwankungen unterliegen, wie die nachfolgende Abbildung zeigt. Diese Differenzen sind in erster Linie darauf zurückzuführen, dass auf Länderebene in der Forschungsförderung eher zeitlich befristete Schwerpunkte anstelle von mehrjährigen Programmen gesetzt werden (zB im Rahmen von Calls).

Abbildung 7: Energieforschungsausgaben der Bundesländer 2010 - 2014

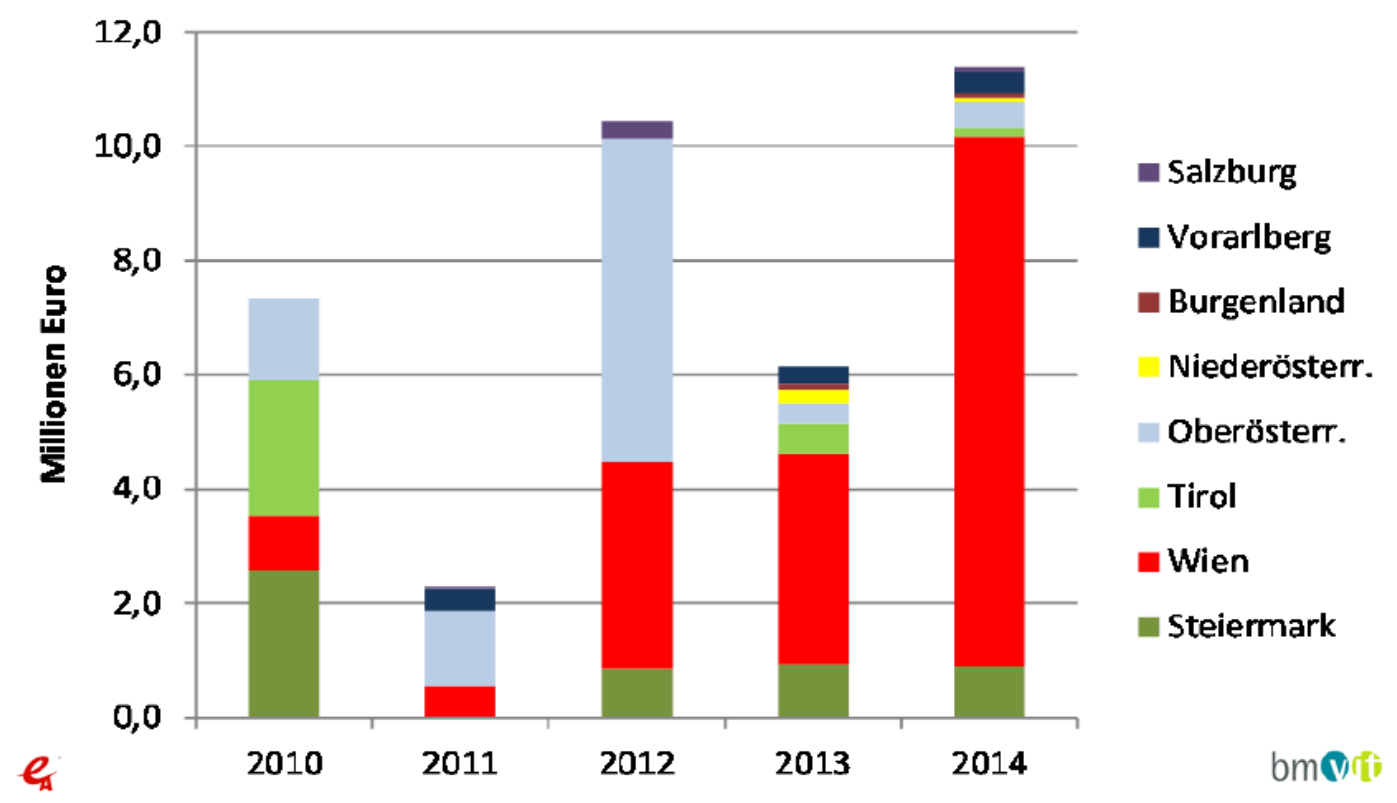

Quelle: BMVIT (2015). Energieforschungserhebung 2014, 68.

Forschungsaktivitäten im Energie- und Umweltbereich werden sowohl in universitären wie auch in außeruniversitären Forschungseinrichtungen gesetzt. Im Hochschulsektor finden sich namhafte Institute, die sich auch intensiv an Haus der Zukunft beteiligt haben (zB TU Wien, TU Graz, FH Joanneum, Uni Innsbruck, Universität Klagenfurt). Unter den außeruniversitären Forschungseinrichtungen sind zunächst das AIT zu nennen, das im Jahr 2014 rund $€$ 15,4 Mio. für Energieforschung aufgewendet hat, wovon rund $€ 1,6$ Mio. auf den Themenbereich "Gebäude und Geräte" entfallen. Weitere außeruniversitäre Forschungseinrichtungen, die zum Teil durch die jeweiligen Länder unterstützt werden, sind u.a. AEE-Institut für Nachhaltige Technologien, Österreichische Energieagentur, Joanneum Research, Österreichische Akademie der Wissenschaften, Energieinstitut an der Johannes Kepler Universität Linz, Niederösterreichische Landesakademie, Energieinstitut Vorarlberg uäm. 
allweise spielen die Länder auch eine wichtige Rolle bei der Einrichtung von thematisch relevanten temporären Forschungseinrichtungen wie Kompetenzzentren (COMET-Programm), CD-Labors, Research Studios etc. Eine Betrachtung der COMET-Zentren und Projekte per Stand Ende 2015 zeigt, dass einige Zentren und Projekte indirekt mit dem Thema Nachhaltiges Bauen verbunden sind (zB Anpassung an den Klimawandel, Mikrosystemtechnik, Bioenergie u.dgl.) und einige wenige unmittel dem Themenfeld zuzuordnen sind:

\section{* K1-Zentren}

Wood Comet. Thema: Holzverbundwerkstoffe und Holzchemie. Träger: Kompetenzzentrum Holz GmbH (Oberösterreich). Laufzeit: 2008-2014

$>$ WOOD. Thema: Next generation materials and processes. Träger: Kompetenzzentrum Holz GmbH (Oberösterreich). Laufzeit: 2015-2018

\section{* K-Projekte}

$>$ Future Building. Thema: Entwicklung nachhaltiger Gebäudekomponenten und -systeme. Träger Donau-Universität Krems, Department für Bauen und Umwelt. Laufzeit: 2009-2014

$>$ focus_solid_timber_solutions. Thema Bausysteme der Holzmassivbauweise in Brettsperrholz, Derivate mit Fokus auf Materialeinsparung, Tragmodelle für die Platten- und Scheibenbeanspruchung, Optimierung Pressparameter, Fügung. Träger: Holz.Bau Forschungs GmbH Steiermark. Laufzeit 20132016

$>$ holz.bau forschungs gmbh - das Kompetenzzentrum für Holzbau und Holztechnologie. Thema Holzbau und Holztechnologie, Verbesserung von Produktionsprozessen, Material- und Strukturmodellierung. Träger: holz.bau forschungs gmbH Steiermark. Laufzeit: 2008-2012

$>$ IPOT Intelligent Photovoltaic module Technologies. Thema: Photovoltaik, PV Modultechnologie, Messtechnik. Träger: CTR - Carinthian Tech Research AG Kärnten. Laufzeit: 2010-2014

MPPF Multifunctional Plug \& Play Facade: Thema: Fassaden mit multifunktionellen Technologien, Vorfertigung, Photovoltaik, Solarthermie, Kontrollsysteme. Träger: Hans Höllwart Forschungszentrum für integrales Bauwesen AG Steiermark. Laufzeit: 2008-2013

Um den Transfer zwischen Forschung und Wirtschaft zu unterstützen, hat sich das Instrument der Clusterinitiativen in Österreich seit Mitte der 1990er Jahre etabliert. Vielfach werden die Clusterinitiativen zum überwiegenden Teil aus Landesmitteln finanziert und sind organisatorisch bei Landesagenturen verankert. Acht Cluster mit knapp 1.200 Partnerbetrieben sind im Themenbereich „Holz, Möbel, 
Wohnen, Hausbau“ aktiv, etwa der Bau.Energie.Umwelt.Cluster Niederösterreich und der Möbel- und Holzbaucluster Oberösterreich sowie die Holzcluster Salzburg, Steiermark und Tirol. Der Bau.Energie.Umwelt.Cluster Niederösterreich setzt einen expliziten Themenschwerpunkt auf Nachhaltiges Bauen und Sanieren und hat dazu wichtige Aktzente in Österreich gesetzt. Weitere 10 Cluster und Netzwerke mit rund 1.000 Partnerbetrieben sind dem Themenfeld "Ökoenergie und Umwelt" zuzuordnen, wo vereinzelt auch Veranstaltungen, Projekte und Initiativen im Baubereich angeboten werden (u.a. Cluster Erneuerbare Energien Tirol, Eco World Styria, Umwelttechnik-Cluster Oberösterreich).

Ein wichtiges Steuerungsinstrument in Hinblick auf die Bautätigkeiten im Wohnbau ist die Wohnbauförderung, die in den Wirkungsbereich der Länder fällt und dementsprechend unterschiedlich ausgestaltet ist. Die Wohnbauförderung hat maßgeblich bewirkt, dass die energetischen Standards im Wohnbau in Österreich in den letzten Jahren deutlich verbessert wurden. Kriterien der Energieeffizienz bis hin zum Passivhausstandard wurden in den 2000er Jahren sukzessive in den Wohnbauförderungen der Bundesländer berücksichtigt - sei es im Sinne von Ausschließungskriterien oder durch Zuschläge für besonders energieeffiziente Gebäude.

Im Zuge der Umsetzung der nationalen Klimastrategie wurden 2005 und 2009 Bund-Länder-Vereinbarungen zum Klimaschutz im Wohnbau getroffen. Darin wurde festgehalten, dass Maßnahmen zur Reduzierung der Treibhausgasemissionen im Gebäudesektor getroffen werden sollen. Auf dieser Grundlage wurden entsprechende Kriterien in den Wohnbauförderungsrichtlinien der Länder verankert (zB Orientierung am Heizwärmebedarf, Anreize für Passivhausstandard im Neubau, weitergehender Ausschluss fossiler Energieträger etc.). 


\section{OUTPUT: Ergebnisse des Programms}

Der dritte Abschnitt des Evaluierungsberichtes beleuchtet den OUTPUT des Programms Haus der Zukunft, also die unmittelbar und kausal mit dem INPUT zusammenhängenden Ergebnisse. Hierzu zählen neben den geförderten Projekten auch die Begleitmaßnahmen. Neben einer umfassenden Auswertung von Programmdaten werden insbesondere die Leitprojekte als "Leuchttürme“ von Haus der Zukunft näher betrachtet.

\subsection{Auswertung der Programmdaten}

Die Auswertung von Programmdaten ermöglicht einen detaillierten Einblick in die Forschungs- und Innovationsaktivitäten, die im Rahmen von Haus der Zukunft ermöglicht wurden.

Grundsätzlich gibt es eine Fülle an Informationen zum Programm und den geförderten Projekten, allerdings liegen aufgrund der langen Laufzeit des Programms keine durchgängigen und einheitlich strukturierten Programmdaten vor, insbesondere zu den ersten drei Ausschreibungen von Haus der Zukunft. Der unvollständigen und uneinheitlichen Datenbasis sind auch kleine Abweichungen in der nachfolgenden Darstellung von Programmdaten aus unterschiedlichen Perspektiven geschuldet. Ab der 3. Ausschreibung von Haus der Zukunft hat die FFG die formale Abwicklung aller Projekte, die durch Haus der Zukunft gefördert wurden, vorgenommen. Daher liegen ab diesem Zeitpunkt detaillierte Daten der FFG vor. Dabei wird zwischen den beiden Programmphase "Haus der Zukunft" und "Haus der Zukunft plus" unterschieden.

\subsubsection{Projektanträge und -genehmigungen}

In Summe wurden ab Erhebung der FFG 425 Projekte genehmigt, die aus 1.095 Projektanträgen hervorgegangen sind. Die Genehmigungsquote liegt daher bei 40 $\%$, was angesichts der Fülle an Projektanträgen einen hohen Wert darstellt.

Die Anzahl an Projektanträgen und -genehmigungen weist deutliche Schwankungen auf, was auf die unterschiedlichen Instrumente, Themenschwerpunkte und Ausschreibungsbudgets zurückzuführen ist. Beachtenswert ist die hohe Anzahl an Projektanträgen bereits bei der ersten Ausschreibung 1999 - dies unterstreicht die Beobachtung, dass der Zeitpunkt des Programmstarts offensichtlich richtig gewählt und ein entsprechendes Potenzial in Österreich gegeben war. 
Programmevaluierung Haus der Zukunft 1999 - 2013

Abbildung 8: Projektanträge und - genehmigungen Haus der Zukunft

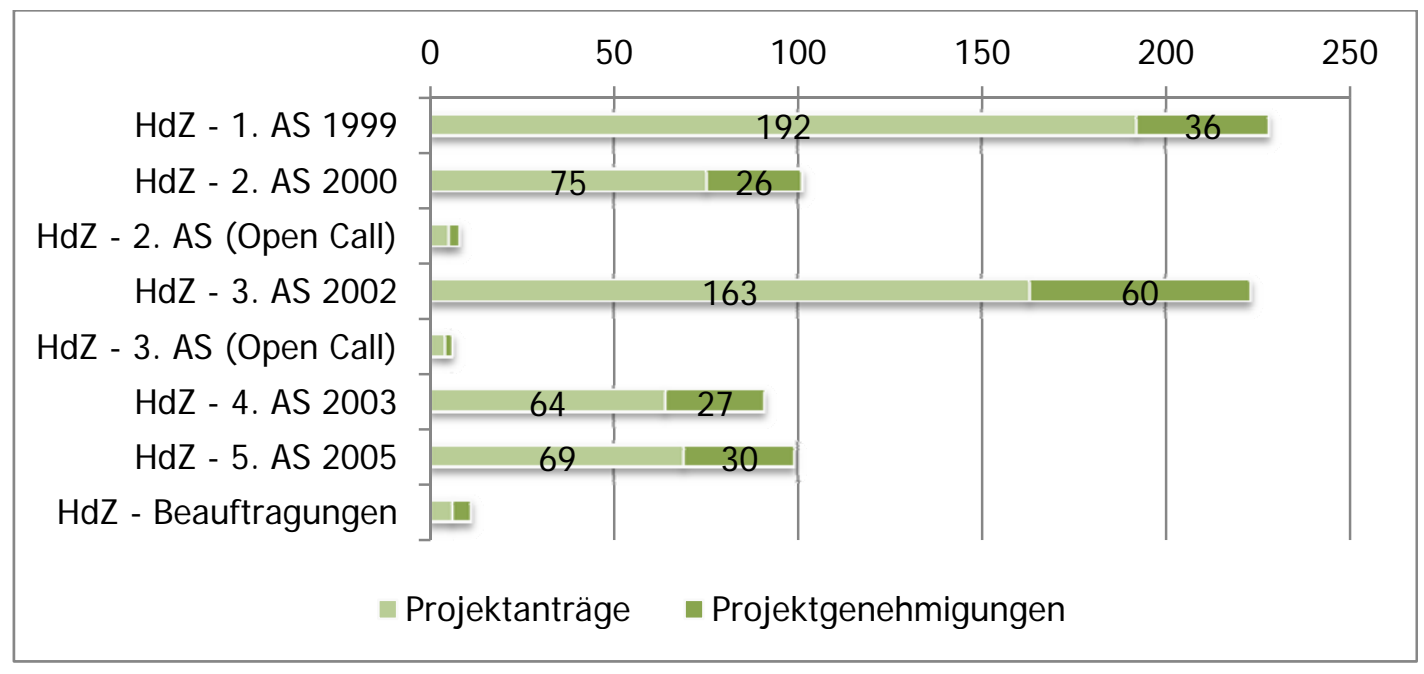

Quelle: TiG, BMVIT, FFG

Das Interesse an den Programmausschreibungen blieb während der gesamten Laufzeit sehr hoch. Deutlich sichtbar ist die Zunahme der Bedeutung von Beauftragungen und Transfermaßnahmen (Überleitungsprojekte, Begleitmaßnahmen, Veranstaltungen/Workshops, Direktvergaben des BMVIT u.ä.).

Abbildung 9: Projektanträge und - genehmigungen Haus der Zukunft plus

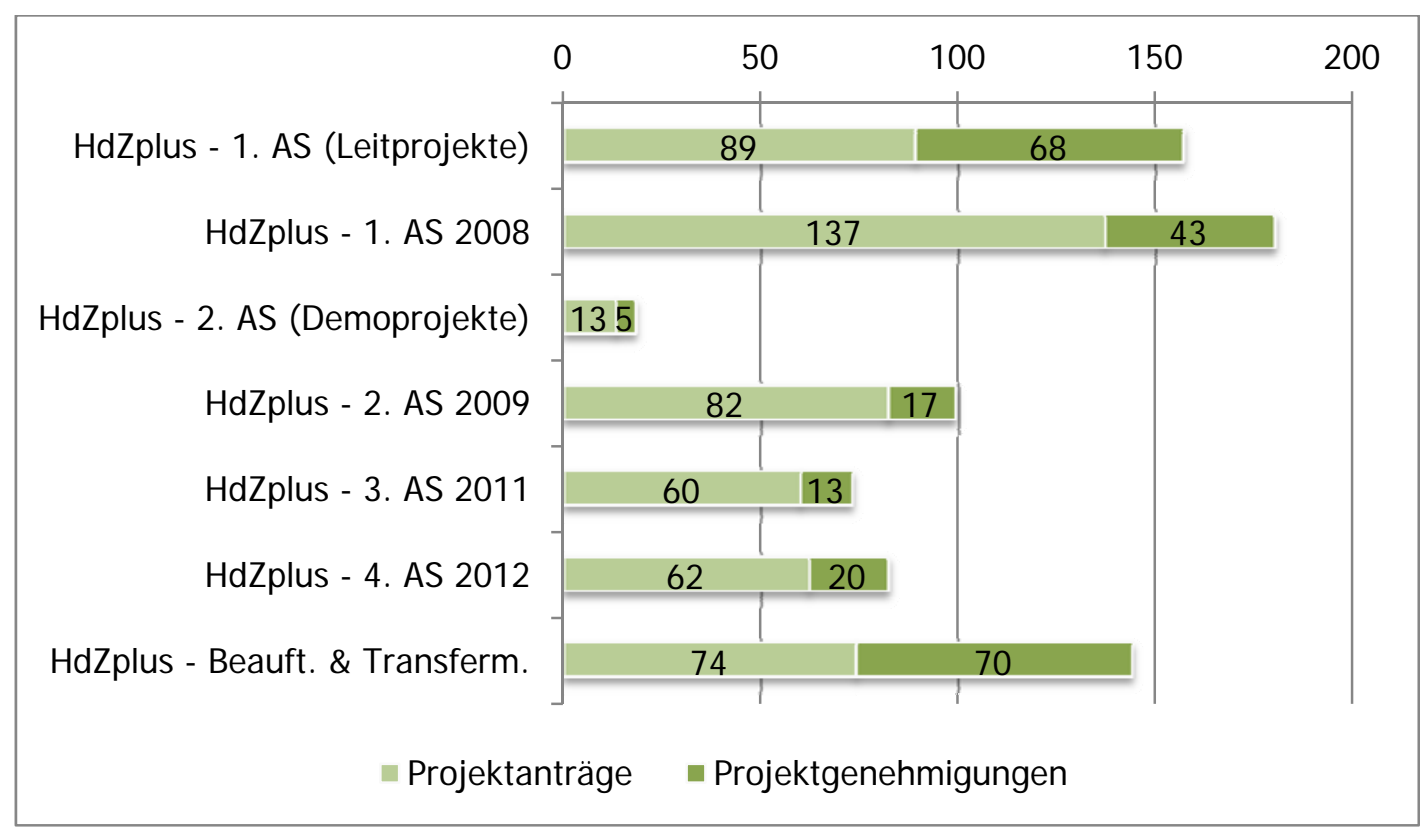

Quelle: FFG 
Inklusive der dritten Ausschreibung 2002 sind insgesamt 916 Beteiligungen an genehmigten Projekten festzuhalten. Im Schnitt waren 2,12 Partner an einem Haus der Zukunft-Projekt beteiligt.

Abbildung 10: Eingereichte und genehmigte Beteiligungen Haus der Zukunft (ab 3. AS)

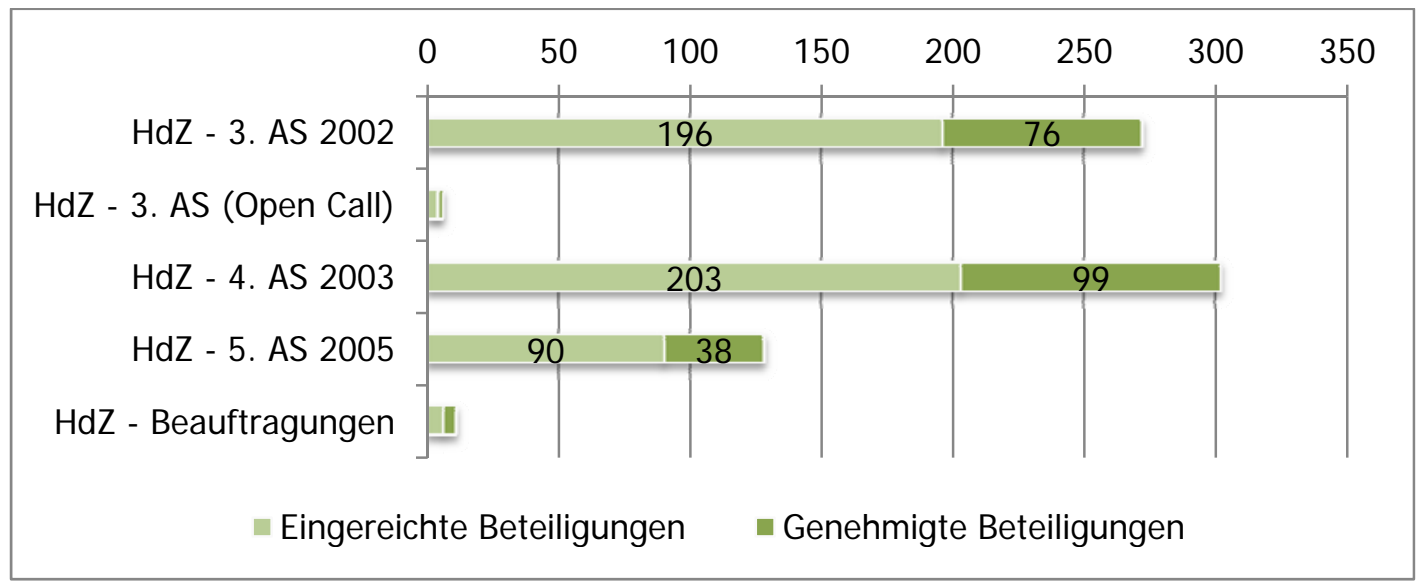

Quelle: TiG, BMVIT, FFG

Über die Programmlaufzeit ist eine Zunahme der Beteiligung sowohl in absoluten Zahlen wie auch in Relation zur Anzahl der Projekte zu beobachten: In der ersten Programmphase Haus der Zukunft waren 220 Akteure beteiligt (durchschnittlich 1,16 Partner pro Projekt) - in der zweiten Programmphase insgesamt 696 Akteure (durchschnittlich 2,86 Partner pro Projekt).

Abbildung 11: Eingereichte und genehmigte Beteiligungen Haus der Zukunft plus

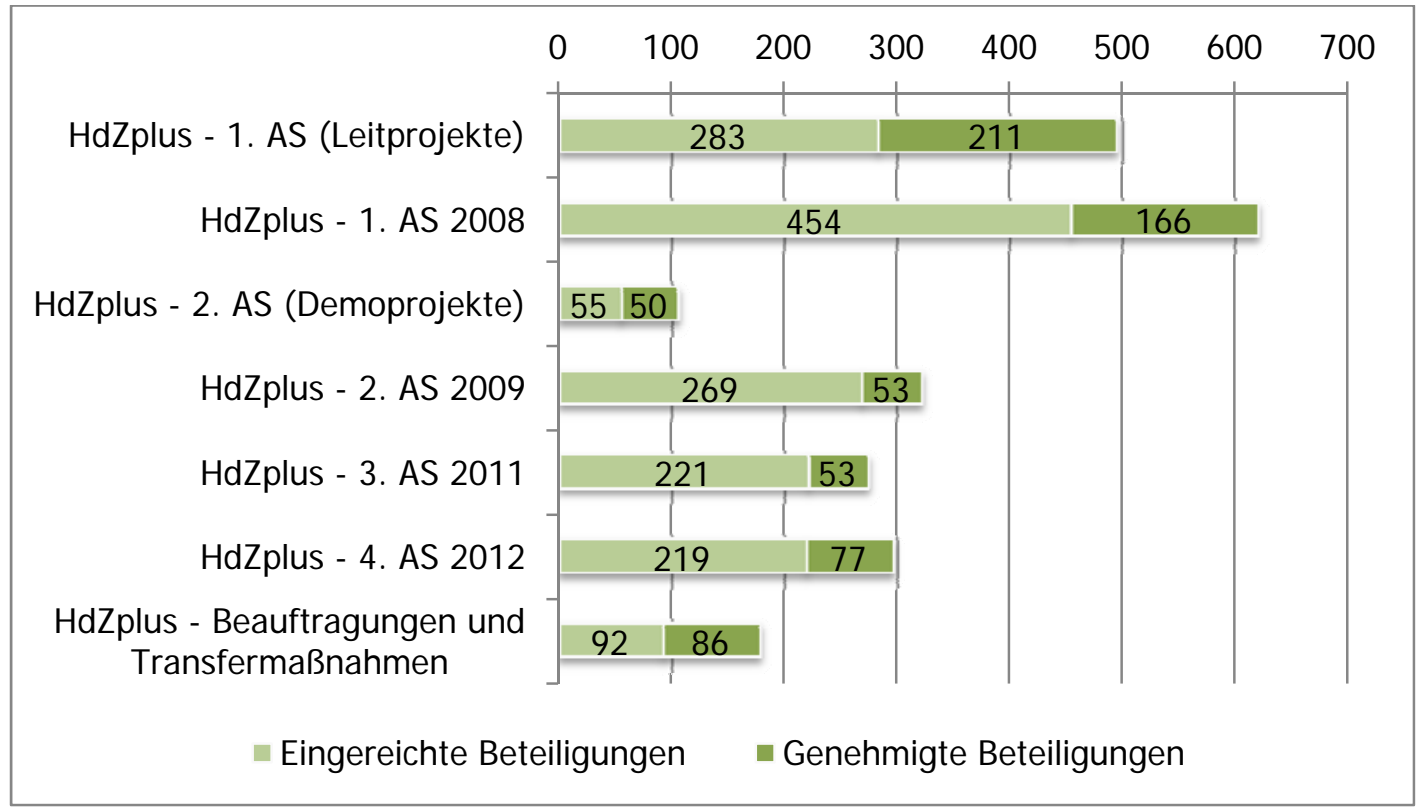

Quelle: FFG 
Programmevaluierung Haus der Zukunft 1999 - 2013

\subsubsection{Projektförderungen}

Die nachfolgende Tabelle gibt einen Überblick über die beantragten Gesamtkosten bzw. Förderung sowie die genehmigten Gesamtkosten bzw. Förderung ab der dritten Ausschreibung von "Haus der Zukunft":

Tabelle 2: Beantragte und genehmigte Gesamtkosten bzw. Förderung im Überblick

\begin{tabular}{|c|c|c|c|c|}
\hline $\begin{array}{l}\text { Ausschrei- } \\
\text { bung }\end{array}$ & $\begin{array}{l}\text { Beantragte } \\
\text { Gesamtkosten }\end{array}$ & $\begin{array}{l}\text { Beantragte } \\
\text { Förderung }\end{array}$ & $\begin{array}{l}\text { Genehmigte } \\
\text { Gesamtkosten }\end{array}$ & $\begin{array}{l}\text { Genehmigte } \\
\text { Förderung }\end{array}$ \\
\hline $\begin{array}{l}\mathrm{HdZ}-3 . \mathrm{AS} \\
2002^{4}\end{array}$ & 39.636 .780 & 30.895 .380 & 11.942 .548 & 8.142 .930 \\
\hline $\begin{array}{l}\mathrm{HdZ}-3 . \text { AS } \\
\text { (Open Call) }\end{array}$ & 954.200 & 463.600 & 431.259 & 215.600 \\
\hline $\begin{array}{l}\mathrm{HdZ}-4 . \mathrm{AS} \\
2003\end{array}$ & 168.527 .960 & 19.244 .460 & 10.858 .220 & 6.102 .220 \\
\hline $\begin{array}{l}\mathrm{HdZ}-5 . \text { AS } \\
2005\end{array}$ & 209.118 .940 & 50.603 .440 & 23.232 .027 & 14.460 .750 \\
\hline $\begin{array}{l}\mathrm{HdZ} \text { - } \\
\text { Beauftragungen }\end{array}$ & 399.151 & 379.151 & 248.287 & 248.287 \\
\hline Summe HdZ & 418.637.031 & 101.586 .031 & 46.712.341 & 29.169.787 \\
\hline $\begin{array}{l}\text { HdZplus - 1. AS } \\
\text { (Leitprojekte) }\end{array}$ & 51.046 .290 & 24.840 .658 & 34.703 .472 & 15.343 .841 \\
\hline $\begin{array}{l}\text { HdZplus - 1. AS } \\
2008\end{array}$ & 269.009 .973 & 83.365 .819 & 15.771 .336 & 11.841 .842 \\
\hline $\begin{array}{l}\text { HdZplus - 2. AS } \\
\text { (Demoprojekte) }\end{array}$ & 20.213 .161 & 7.913 .375 & 7.673 .770 & 3.410 .478 \\
\hline $\begin{array}{l}\text { HdZplus - 2. AS } \\
2009\end{array}$ & 63.344 .121 & 32.764 .456 & 9.620 .876 & 5.012 .503 \\
\hline $\begin{array}{l}\text { HdZplus - 3. AS } \\
2011\end{array}$ & 154.488 .836 & 26.234 .478 & 5.337 .303 & 4.221 .845 \\
\hline $\begin{array}{l}\text { HdZplus - 4. AS } \\
2012\end{array}$ & 36.606 .231 & 24.565 .835 & 10.089 .234 & 6.255 .482 \\
\hline $\begin{array}{l}\text { HdZplus - } \\
\text { Beauftragungen } \\
\text { und Transfer- } \\
\text { maßnahmen }\end{array}$ & 13.485 .039 & 4.469 .350 & 8.076 .733 & 4.602 .649 \\
\hline $\begin{array}{l}\text { Summe } \\
\text { HdZplus }\end{array}$ & 608.193.651 & 204.153.971 & 91.272 .724 & 50.688 .640 \\
\hline $\begin{array}{l}\text { Summe HdZ \& } \\
\text { HdZplus }\end{array}$ & 1.026 .830 .682 & 305.740 .002 & 137.985 .065 & 79.858.427 \\
\hline
\end{tabular}

Quelle: FFG

4 Keine validen Angaben zur ersten und zweiten Ausschreibung von Haus der Zukunft verfügbar 
Aus der tabellarischen Darstellung werden auf den ersten Blick die Dimensionen des Förderprogramms deutlich: In Summe wurden Projekte mit einem Gesamtvolumen von über einer Milliarde Euro beantragt, die beantragte Förderung belief sich insgesamt auf $€ 306$ Mio. Es konnten davon Projekte mit einem Gesamtvolumen von $€ 138$ Mio. mit einer Förderung in Höhe von knapp $€ 80$ Mio. unterstützt werden. Dies sind deutliche Zeichen für die Potenziale von Forschung und Innovation im Bereich Nachhaltiges Bauen in Österreich.

Die Höhe der Förderung pro Ausschreibung war durch die Ausschreibungsbudgets im Wesentlichen vorgegeben, jedoch waren gewisse Umschichtungen möglich. Beachtenswert sind die Volumina in der ersten Programmphase, wo bis zu $€$ 14 Mio. bei einer Ausschreibung vergeben werden konnten. Den Beginn der zweiten Programmphase markiert die Ausschreibung von Leitprojekten, die mit über $€$ 15 Mio. unterstützt wurden. Im Anschluss an die erste reguläre Ausschreibung der zweiten Programmphase sind die Ausschreibungsbudgets merklich zurückgegangen - die letzte Ausschreibung im Jahr 2012 umfasste eine ungefähr gleich große Summe an Förderungen wie jene im Jahr 2003.

Aufgrund der begrenzten Förderbudgets konnte nur knapp ein Viertel der beantragten Förderungen genehmigt werden. Besonders hoch war die Genehmigungsrate bei den Ausschreibungen für Demonstrations- und Leitprojekte, was auf das zweistufige Vergabeverfahren sowie generell den höheren Aufwand im Zusammenhang mit der Beantragung eines kooperativen Innovations- und Investitionsprojektes zurückzuführen ist.

Die Förderung von Transfermaßnahmen und Beauftragungen im Kontext des Programms (Überleitungsprojekte, Begleitmaßnahmen, Veranstaltungen und Workshops, uäm.) nahm in der ersten Programmphase nur einen marginalen Anteil ein. In der zweiten Programmphase wurden dafür rund $€$ 4,6 Mio. aufgewendet, was etwas über $5 \%$ der gesamten Fördersumme ausmacht.

Eine Detailbetrachtung der genehmigten Gesamtkosten bzw. Förderung in der zweiten Programmphase zeigt, dass die erste Ausschreibung von Leitprojekten einen großen Anteil innerhalb des Programms eingenommen hat. Das Verhältnis von beantragten zu genehmigten Förderungen weicht hier etwas von den anderen Ausschreibungen ab, da bei Leitprojekten nur bestimmte Teile des Projektes bzw. die Mehrkosten für innovative Technologien und Lösungen bei Baumaßnahmen gefördert wurden. Ähnliches gilt für die Ausschreibung von Demonstrationsvorhaben. 
Abbildung 12: Genehmigte Gesamtkosten / Förderung Haus der Zukunft plus

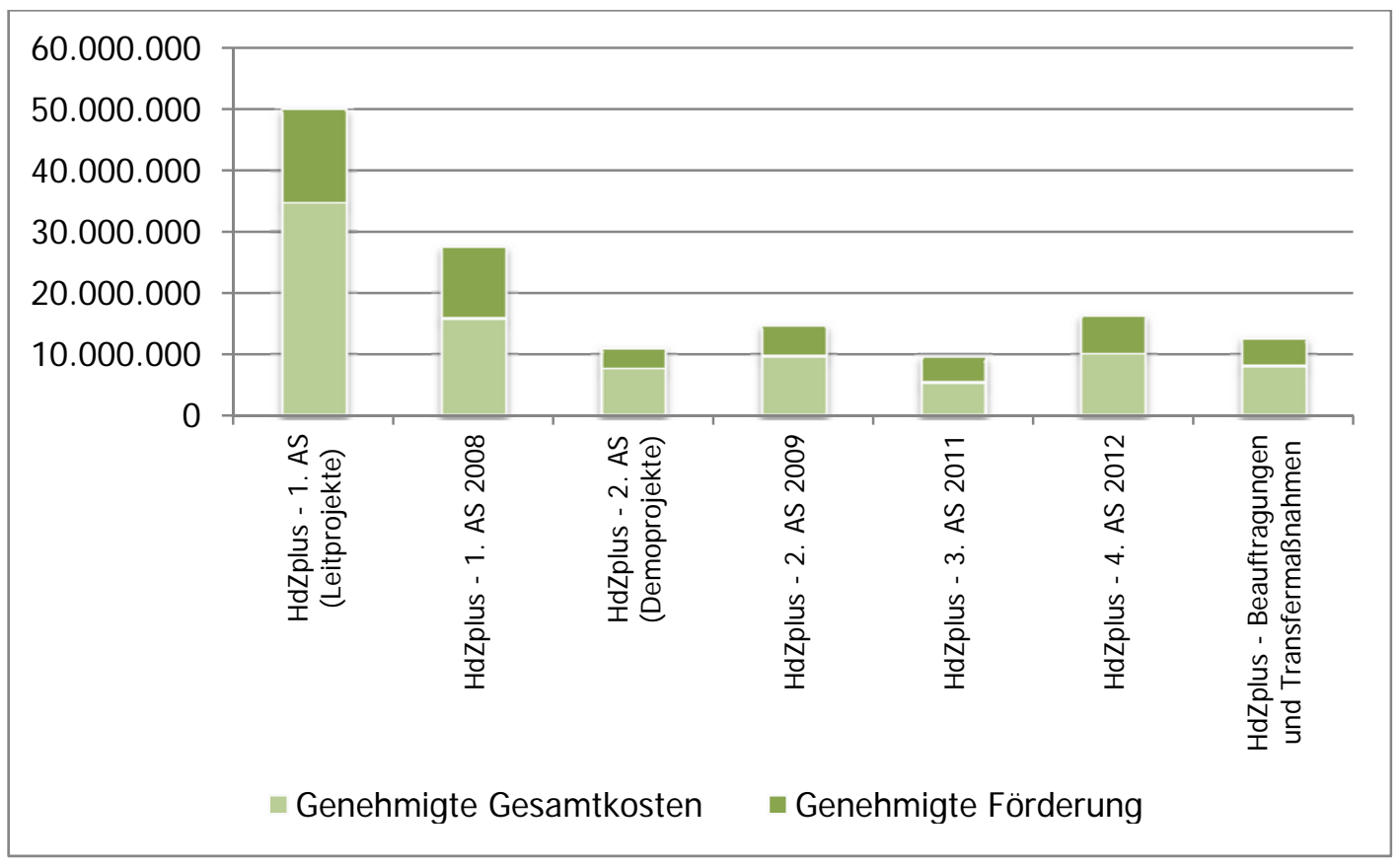

Quelle: FFG

Eine Betrachtung der Förderungen nach Jahr der Genehmigung zeigt die große Bedeutung der Leitprojekte, die großteils in den Jahren 2009 und 2010 positiv beschieden wurden. Weitere Einreichungen von Subprojekten bzw. anderen programmrelevanten Vorhaben waren darüber hinaus möglich, sodass auch in den Jahren 2014 und 2015 Genehmigungen kleineren Ausmaßes vorgenommen wurden.

\section{Abbildung 13: Förderung nach J ahr der Genehmigung}

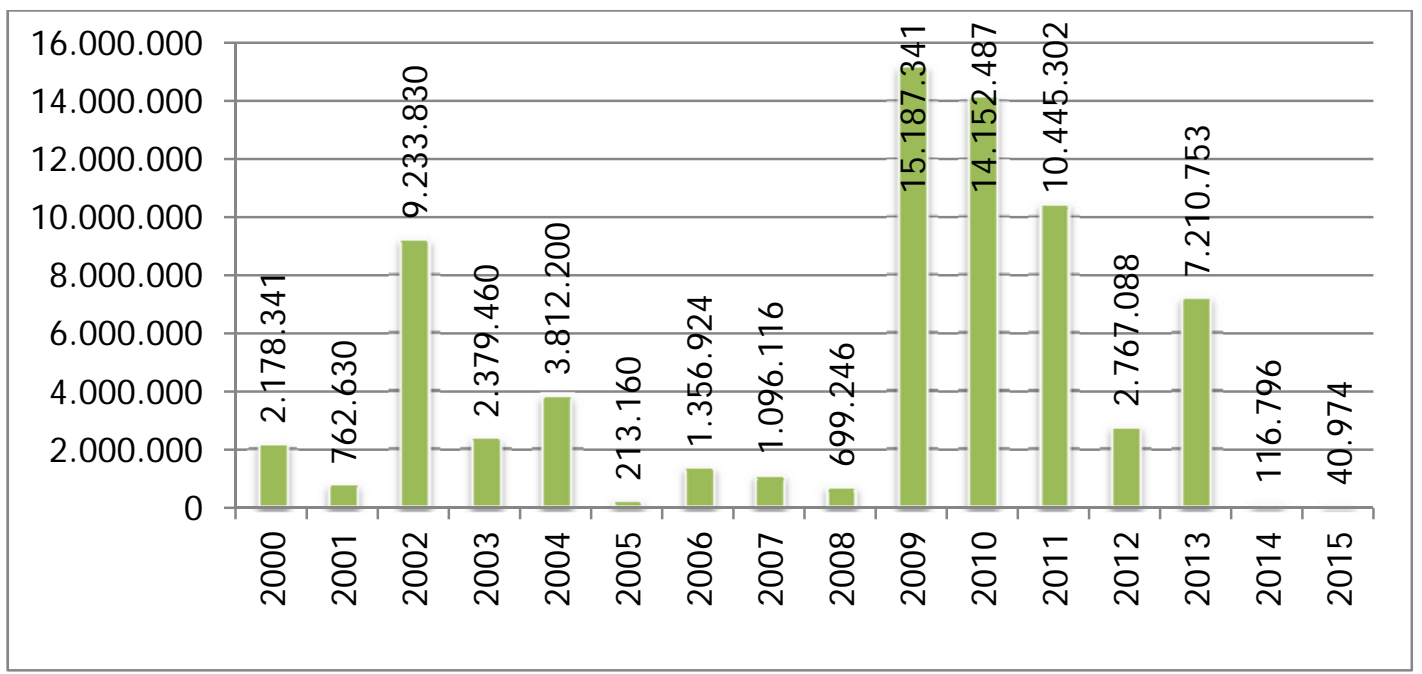




\subsubsection{Struktur der Fördernehmer}

Die ausführlichen Programmdaten der FFG und der AWS beinhalten auch Informationen zu den Fördernehmern, die in aggregierter und anonymisierter Form ausgewertet werden können. Die Auswertung nach dem Organisationstyp zeigt, dass insbesondere die Unternehmen mit dem Programm erreicht werden konnten. In der ersten Programmphase machten sie rund die Hälfte aller Begünstigten aus, in der zweiten Programmphase rund ein Drittel.

Während in der ersten Programmphase die zweitgrößte Gruppe der Fördernehmer noch außeruniversitäre Einrichtungen waren, so waren es in der zweiten Phase Forschungskonsortien mit Unternehmensbeteiligung - die acht Leitprojekte machen dabei nur einen minimalen Anteil aus. Das unterstreicht die zunehmende Kooperationsorientierung während der Programmlaufzeit. Nichtsdestotrotz haben sich Universitäten und außeruniversitäre Einrichtungen maßgeblich an geförderten Projekten beteiligt, auch in der zweiten Programmphase.

Ein ähnliches Bild zeigt sich bei einer Betrachtung der genehmigten Förderung nach Organisationstyp. Unternehmen nehmen hier einen geringeren Anteil an, da nicht-gewinnorientierte Institutionen in der Regel einen höheren Fördersatz beanspruchen können. In der zweiten Phase werden beim Typus der Forschungskonsortien mit Unternehmensbeteiligung die kapitalintensiven Leitprojekte deutlich sichtbar. Die prozentuale Aufteilung ist in Verbindung mit den absoluten Zahlen zu sehen - so haben beispielsweise Unternehmen in der zweiten Programmphase um 50 \% mehr an Geldern erhalten, was jedoch einen geringen Anteil an der Gesamtförderung darstellt. 
Programmevaluierung Haus der Zukunft 1999 - 2013

Abbildung 14: Beteiligte an genehmigten Projekten nach Organisationstyp Haus der Zukunft

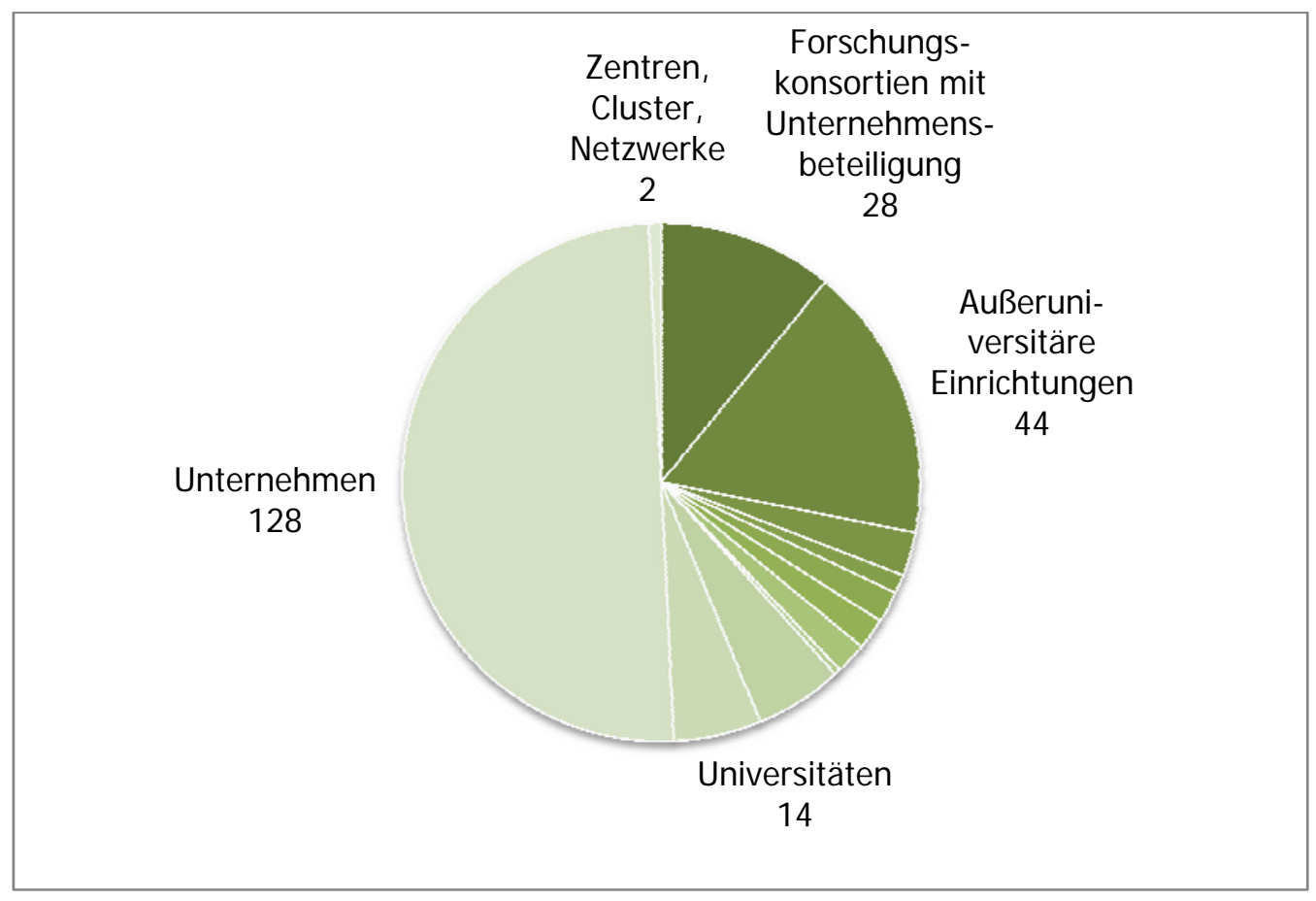

Quelle: FFG

Abbildung 15: Beteiligte an genehmigten Projekten nach Organisationstyp Haus der Zukunft plus

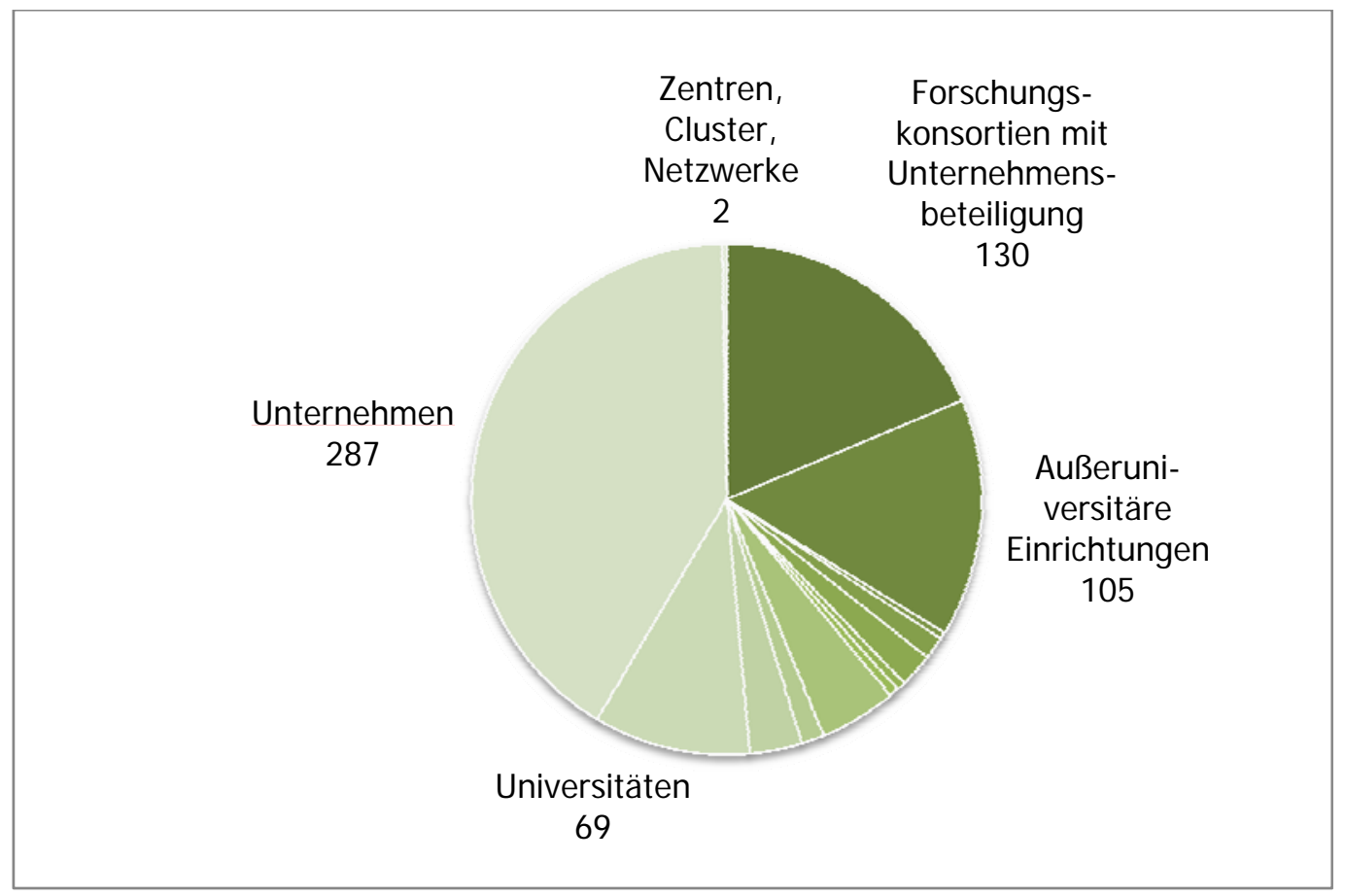

Quelle: FFG 
Abbildung 16: Genehmigte Förderung nach Organisationstyp Haus der Zukunft

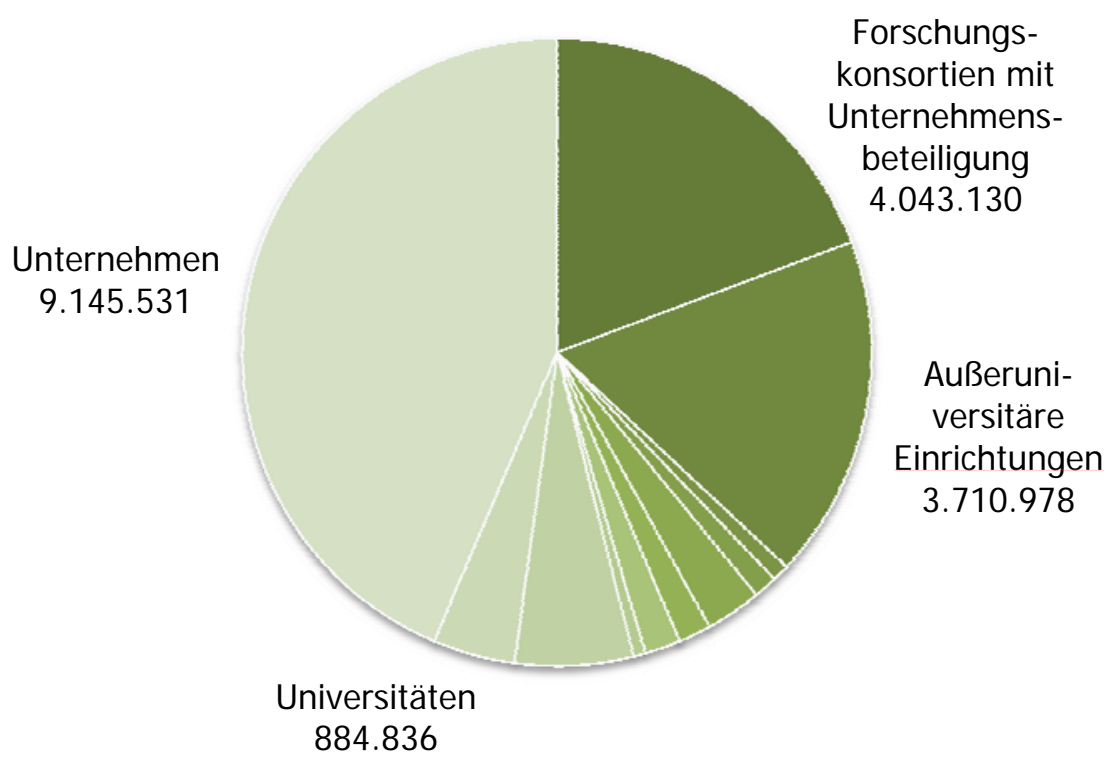

Quelle: FFG

Abbildung 17: Genehmigte Förderung nach Organisationstyp Haus der Zukunft plus

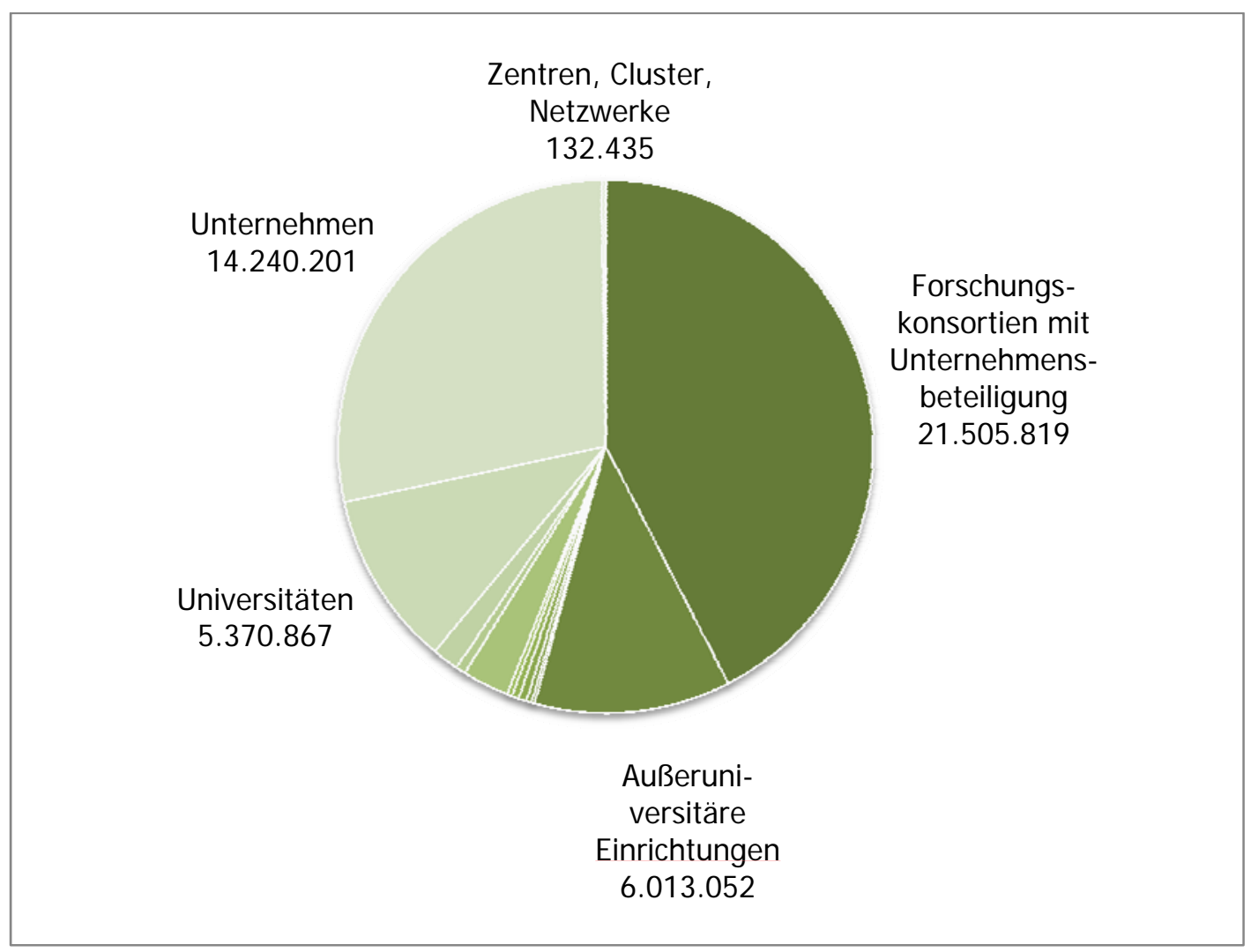

Quelle: FFG 
Bei geförderten Projekten waren die meisten Unternehmen der Branche "Architektur- und Ingenieurbüros" sowie „Forschung und Entwicklung“ zugeordnet. Dies ist wenig überraschend, da in diesen Branchen technische und wissenschaftliche Dienstleistungen erbracht werden, die für Forschungs- und Innovationsvorhaben eine zentrale Rolle spielen. Bemerkenswert ist dies allerdings deshalb, weil wissensbasierte Dienstleister - mit Ausnahme von IKT-Dienstleistern - üblicherweise kaum an FTI-Förderprogrammen teilnehmen können. Interessenvertretungen, Unternehmen im Grundstücks- und Wohnungswesen sowie Unternehmensberatungsbetriebe waren ebenfalls zahlreich an Projekten beteiligt. Relativ gering fällt die Beteiligung von Unternehmen der Bauwirtschaft im engeren Sinne aus.

\section{Abbilldung 18: Beteilligte Unternehmen an geförderten Projekten nach NACE-Klassen}

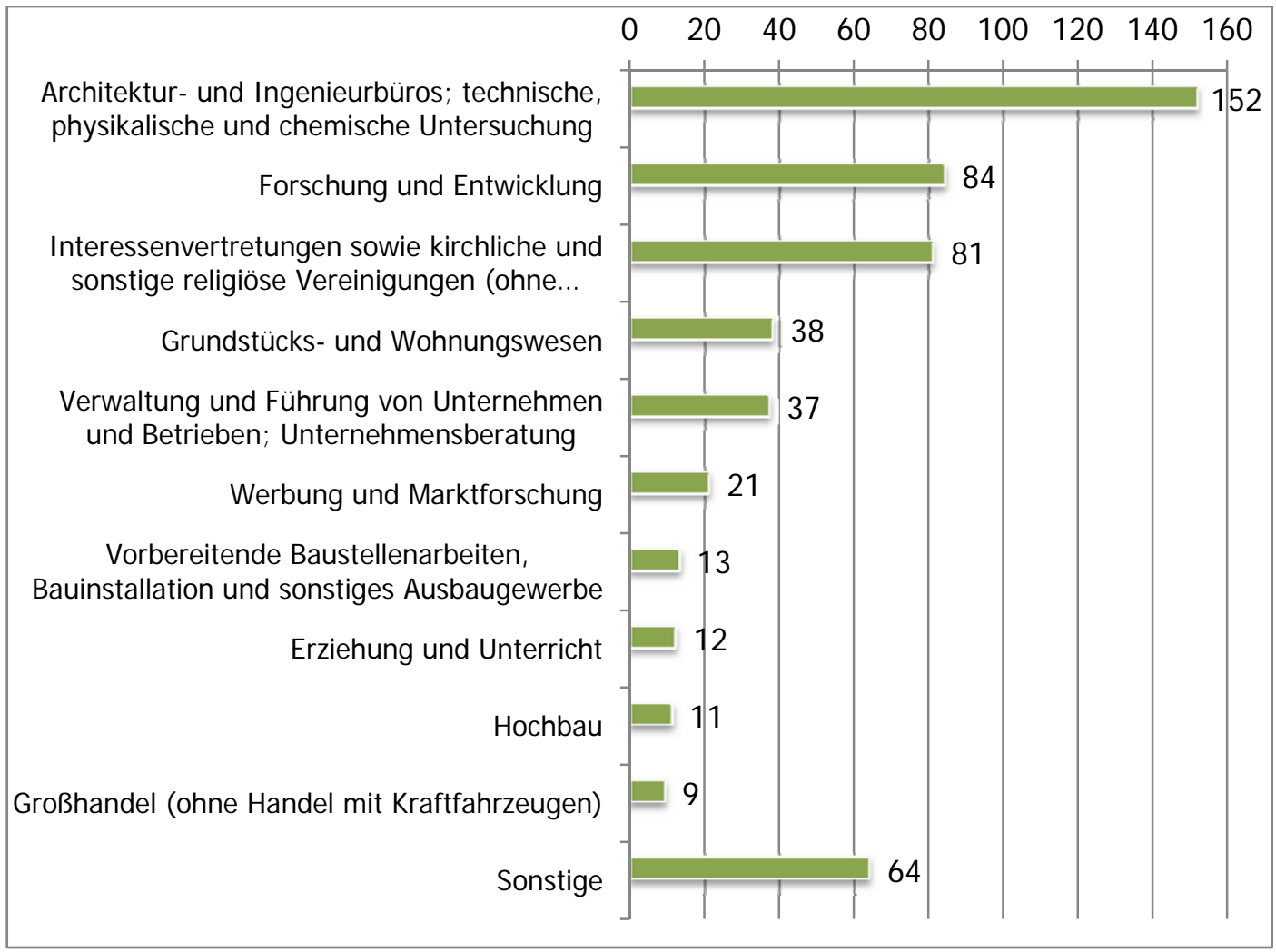

\section{Quelle: FFG}

Als Programm eines österreichischen Ministeriums richtete sich Haus der Zukunft vorrangig an österreichische Einrichtungen. Um den grenzüberschreitenden Wissens- und Technologietransfer zu unterstützen, war fallweise die Beteiligung ausländischer Partner möglich. In der zweiten Programmphase wurden 1.554 Projekt-Beteiligungen aus Österreich eingereicht, weiters 22 aus Deutschland, 7 aus der Schweiz, 3 aus der Slowakei und Italien, 2 aus Frankreich und jeweils eine aus Tschechien und Slowenien. Diese Beteiligungen wurden großteils auch unterstützt, in Summe wurden ausländische Projektpartner mit $€ 322.000$ gefördert. 
Innerhalb Österreichs entfielen in beiden Programmphasen die meisten genehmigten Gesamtkosten bzw. Förderungen auf Wien, gefolgt von den Bundesländern Steiermark, Nieder- und Oberösterreich. Hierbei ist gerade in der zweiten Programmphase zu berücksichtigen, dass die Leitprojekte auch in finanzieller Hinsicht deutlich von üblichen Forschungs- und Innovationsprojekten divergieren. Im Schnitt wurde jedes Leitprojekt mit knapp € 2 Mio. gefördert, was sich in einer solchen regionalen Betrachtung zwangsweise niederschlägt.

Abbildung 19: Genehmigte Gesamtkosten und Förderung nach Bundesländern Haus der Zukunft

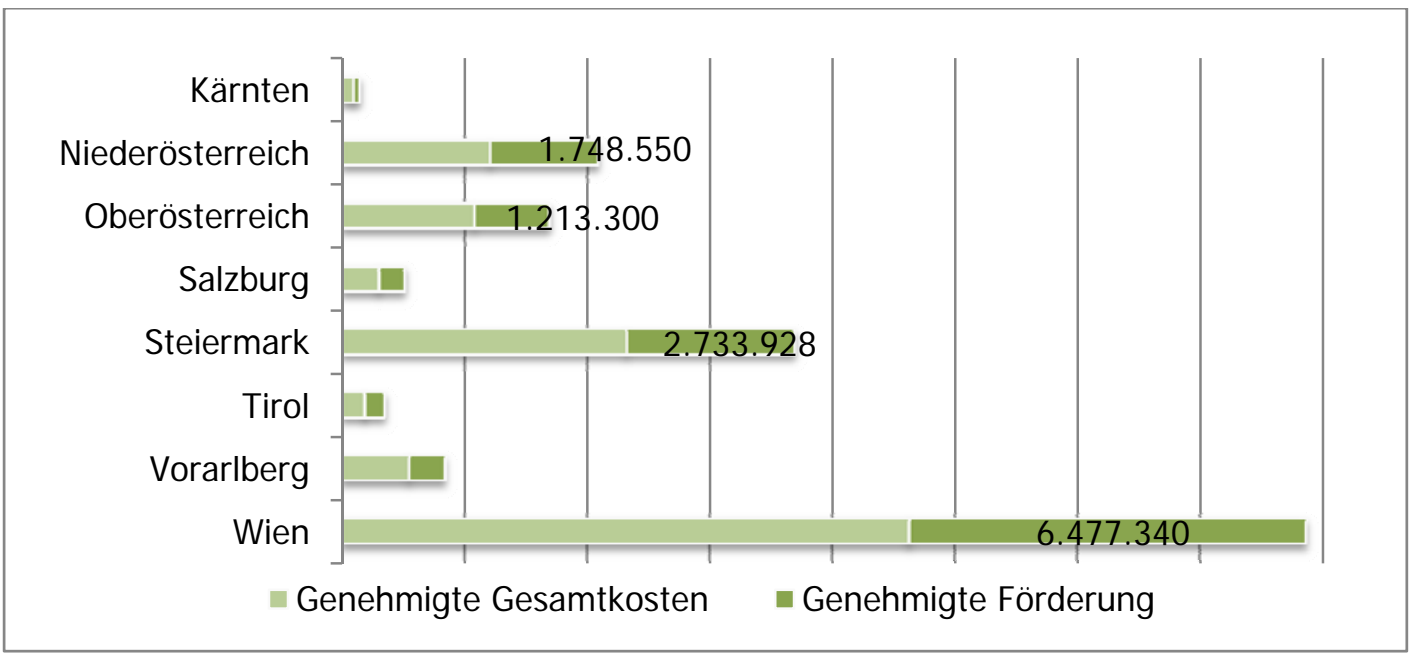

Quelle: FFG

Abbildung 20: Genehmigte Gesamtkosten und Förderung nach Bundesländern Haus der Zukunft plus

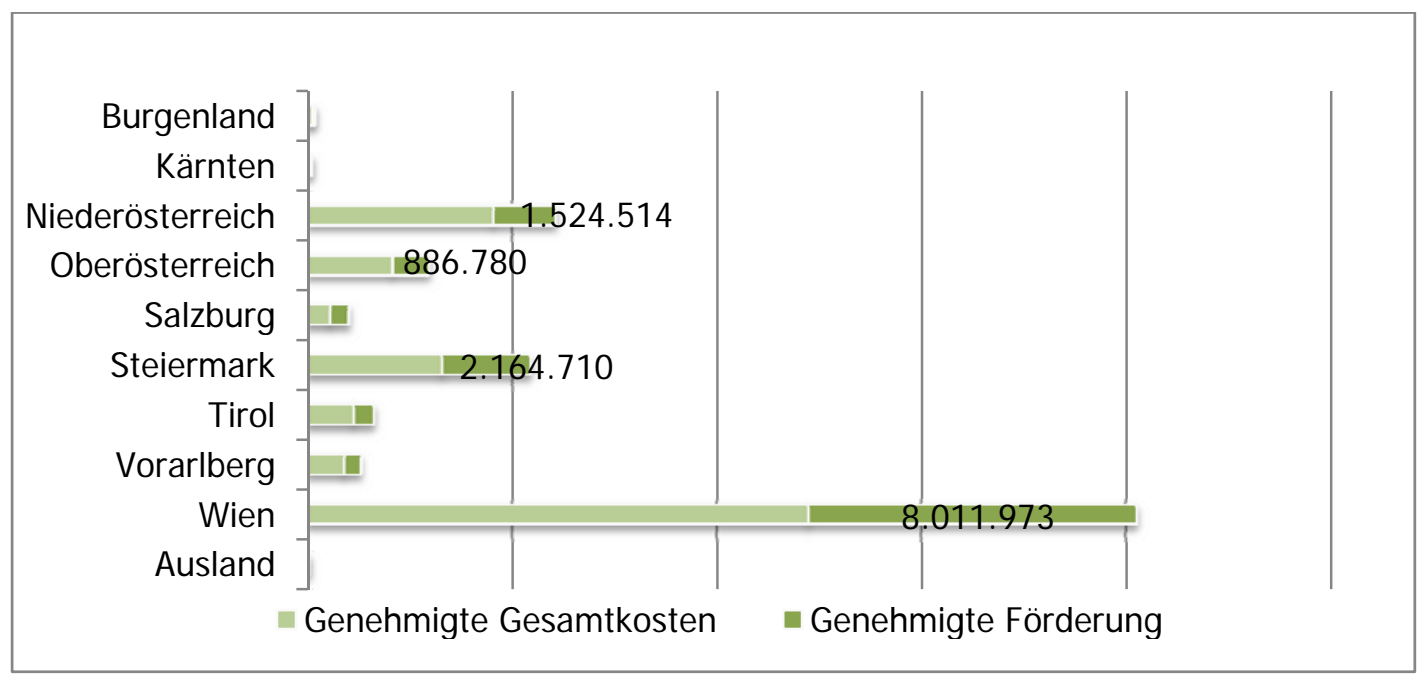

Quelle: FFG 


\subsection{Demonstrationsprojekte}

Das Gros der Haus der Zukunft-Projekte waren klassische Forschungs- und Entwicklungsprojekte, ergänzt durch eine geringere Anzahl an Investitionsprojekten sowie die erwähnten Begleitmaßnahmen. Bereits ab der dritten Ausschreibung wurden auch Demonstrationsprojekte gefördert, bei denen die praktische Umsetzung innovativer Konzepte, Ansätze und Technologien im Vordergrund stand. Damit sollte die Praxistauglichkeit neuer Entwicklungen unter Beweis gestellt und der erzielbare Energieeinsparungseffekt aufgezeigt werden. Daher wurden alle Demonstrationsprojekte einer begleitenden Evaluierung und einem gemeinsamen Monitoring unterzogen. Mit der Erfassung von Energieverbrauchsdaten, der Gebäudebewertung mit dem Tool "Total Quality Building" und der Erhebung der Akzeptanz bei Nutzern wurde überprüft, ob die innovativen Demonstrationsgebäude die Qualitätsansprüche sowie die in sie gesetzten Erwartungen tatsächlich erfüllten.

Die 68 Demonstrationsprojekte sind in unterschiedlichen Regionen Österreichs angesiedelt, wie die folgende Landkarte zeigt. Thematisch sind Demonstrationsprojekte sowohl im Bereich Neubau wie auch der Sanierung zu verorten und gehen vielfach auf vorgelagerte Forschungs- und Entwicklungsprojekte zurück.

Abbildung 21: Landkarte Demonstrationsprojekte

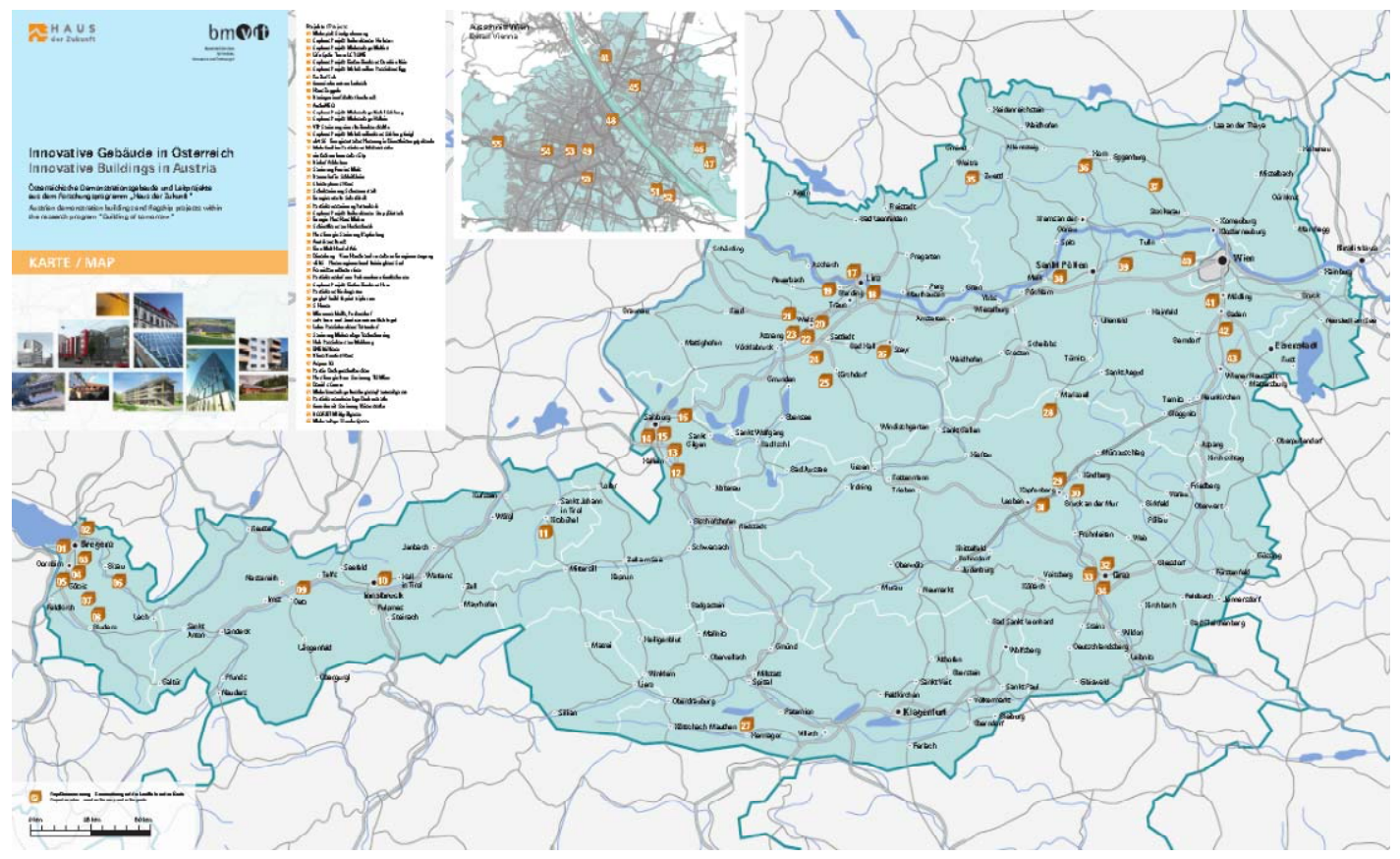

Quelle: http://www.hausderzukunft.at/hdz_pdf/innovative_gebaeude_in_oesterreich _2012_karte.pdf 
Nachfolgend werden einige Beispiele der Demonstrationsgebäude exemplarisch vorgestellt:

LCT ONE Dornbirn: Ein auf dem Rohstoff Holz basierendes Hochhaus in Dornbirn wurde weitgehend industriell vorgefertigt, sodass - bei entsprechender Vorbereitung und Planung - an einem Tag ein ganzes Stockwerk errichtet werden kann. Neben Holz kommen auch massive Materialien wie Beton zum Einsatz, die Außenfassade des 8-stöckigen Gebäudes ist aus Aluminium gefertigt. Der Niedrigstenergiestandard für Neubauten wird in der Praxis um $40 \%$ unterschritten.

* Schiestlhaus: Die auf 2.154 Metern gelegene Schutzhütte in der Hochschwabgruppe wurde ab 2004 abgetragen und durch einen energieautraken Neubau ersetzt. Es handelt sich dabei um das erste hochalpine Passivhaus. Der Baukörper besteht aus einem 2-geschoßigen Holzriegel-Bau. Die Südfassade wurde als Solarfassade gebildet, weiters eine zentrale Be- und Entlüftungsanlage installiert. Die komplette Trinkwasser- und Brauchwasserversorgung erfolgt über natürliche Niederschläge. Das Projekt wurde u.a. mit dem ersten Preis des „Energy Globe“ in der Bundesländerkategorie ausgezeichnet.

* ENERGYbase: Das Kompetenzzentrum für Erneuerbare Energie des Wiener Wirtschaftsförderungsfonds (WWFF) beherbergt auf $7.500 \mathrm{~m}^{2}$ einerseits Unternehmen und andererseite Bildungs- und Forschungseinrichtungen. Das Gebäudekonzept verbindet eine hohe Energieeffizienz mit der umfassenden Nutzung erneuerbarer Energieträger und einem hohen Nutzungskomfort. Grundsätzlich konnte so eine Energieeinsparung von ca. 80 \% und eine Reduktion von ca. 200t/a $\mathrm{CO}_{2}$-Emissionen gegenüber einem konventionellen Gebäude erreicht werden.

* Schulsanierung Schwanenstadt: Die Sanierung mit Zubau der 12-klassigen Hauptschule und 8-klassigen Polytechnischen Schule in Schwanenstadt stellte die erstmalige Sanierung eines öffentlichen Gebäudes auf Passivhaus-Standard dar. Dabei kam ein Maßnahmenmix zum Einsatz (Vorgefertigte Holzwandelement, Dämmung, Wärmebrückenreduzierung, Lüftungsintegration im Bestand, Tagesbelichtung, sommerlicher Wärmeschutz etc.). Das Projekt zeigte die Machbarkeit einer großvolumigen Passivhaussanierung zu vertretbaren Mehrkosten auf und wirkte als Vorbild für weitere Schulsanierungen.

* LISI-Haus: Das Plus-Energiehaus LISI („Living Inspired by Sustainable Innovation“) wurde von einem Team unter Leitung der TU Wien konzipiert. Es verfolgt einen innovativen Bauansatz und gliedert sich in drei Zonen: Servicekern, Wohnbereich und angrenzende Innenhöfe, die durch eine flexible Außenhülle geschlossen werden können. Durch das Zusammenspiel von modularer Leichtbauweise, ökologischen Materialien und erneuerbarer Energie entsteht ein qualitativ hochwertiger, nachhaltiger und leistbarer Wohnbau, der sich an die ver- 
schiedenen Bedürfnisse der Nutzer und Standorte anpassen lässt. Das LiSiHaus wurde 2013 mit dem "Solar Decathlon“ ausgezeichnet - ein Preis des USamerikanischen Energieministeriums für nachhaltige Baukonzepte - und kann in der "Blauen Lagune“ besichtigt werden.

* oh456: Das hocheffiziente Passivhaus im Salzburger Thalgau nutzt lokal vorhandene Ressourcen, um zum Plusenergiegebäude zu werden. Das Bürogebäude wird durch ein benachbartes Kleinwasserkraftwerk und eine Photovoltaikanlage gespeist. Die Energieerträge und -verbräuche werden durch ein zentrales Monitoring erfasst.

* Passivhaustechnologie im sozialen Wohnbau: Dieses viel beachtete Demonstrationsprojekt hat aufgezeigt, dass die für den sozialen Wohnbau erforderlichen niedrigen Baukosten durchaus mit hohen energetischen Standards in Einklang gebracht werden können. In der Utendorfgasse in Wien-Penzing wurde ein mehrgeschossiger sozialer Passivwohnbau mit 39 Wohneinheiten errichtet. Die Mehrkosten für die Passivhausbauweise beliefen sich auf $€ 75 / \mathrm{m}^{2}$ bei Baukosten von $€ 1.055 / \mathrm{m}^{2}$. Diese Innovation war durch die enge Zusammenarbeit von sieben Planungsbüros unterschiedlicher fachlicher Ausrichtung in einem integralen Planungsprozess möglich.

* Klosterneuburg Kierling: Die Sanierung einer Wohnanlage der BUWOG aus den 1970er Jahren erfolgte nach höchsten Maßstäben der Nachhaltigkeit. Zum Einsatz kamen hochwärmegedämmte Fassaden, 3-Scheiben-Verglasung, kontrollierte Be- und Entlüftung mit Wärmerückgewinnung, etc. Bei der Materialauswahl wurde auf emissionsarme Werkstoffe besonderen Wert gelegt.

* Kolpinghaus Salzburg: Bei der Erweiterung des Salzburger Kolpinghauses wurde erstmals in Österreich wird ein 3-Sterne-Hotel in Plusenergie-Standard realisiert. Durch Integration von Photovoltaik, Nutzung der im Abwasser enthaltenen Wärme sowie hohe Ansprüche an einen energieeffizienten Betrieb entstand bei diesem innovativen Vorhaben ein für die ganze Branche beispielgebender Leuchtturm.

Eine besondere Bedeutung kam den acht Leitprojekten zu, da sie in Hinblick auf den Innovationsgehalt, die Sichtbarkeit und die Investitionskosten deutlich höhere Anforderungen als die Demonstrationsprojekte zu erfüllen hatten. Aufgrund dessen werden sie im folgenden Abschnitt näher beschrieben. 


\subsection{Leitprojekte}

Im Rahmen der ersten Ausschreibung der zweiten Programmphase 2008 wurden Leitprojekte gestartet. Dabei handelte es sich um ein neues Projektformat, das aus einem zusammenhängenden Bündel von Einzelprojekten („Subprojekte“) bestand, die - integriert in eine klar definierte Strategie - jeweils ein konkret beschriebenes Ziel verfolgten und von einem gemeinsamen Gesamtprojektmanagement koordiniert wurden. Die Leitprojekte waren vorrangig den Themen PlusEnergie-Siedlungen und Sanierung gewidmet.

Den Programmzielen entsprechend sollten neue Technologien und Innovationen unterstützt und deren Markteinführung bzw. - verbreitung forciert werden. Damit sollte maßgeblich zur Entwicklung einer mit einem nachhaltigen Energiesystem zu vereinbarenden Gebäudekonzeptionierung und zur Erhöhung der Wettbewerbsfähigkeit österreichischer Unternehmen beitragen werden.

Die Einreichung und Beurteilung der Leitprojekte stellte eine Herausforderung dar, da es sich um eine wesentliche Neuerung gegenüber der in "Haus der Zukunft" gewohnten Vorgangsweise handelte. Deshalb wurde eine ständige Jury eingerichtet, welche die Genehmigung bzw. Ablehnung von Subprojektanträgen vornahmen und laufend den Projektfortschritt beurteilten. Die Koordination der Leitprojekte und der ständigen Jury hatte die aws inne.

Die Projektgenerierung - zum Teil auch durch aktive Ansprache von potenziellen Projektträgern - sowie das laufende Coaching der Leitprojekte wurde durch das Managementteam wahrgenommen. Der erstmalige Einsatz dieses innovativen Formats hat in der praktischen Umsetzung eine Reihe von Fragen ergeben. Aus diesem Grund wurden regelmäßig Abstimmungstreffen zwischen den Leitprojektmanagern und dem Managementteam vorgenommen. Einhellig wurde in den Gesprächen im Zuge der Evaluierung betont, dass auf diesem Weg eine effektive Klärung der administrativen Fragen und die optimale Umsetzung der Leitprojekte erzielt werden konnte. Darüber hinaus wurden so ein enger Austausch und eine intensivere Kooperation zwischen den Leitprojekten angestoßen.

Gerade weil die Leitprojekte in mehrfacher Hinsicht Neuland darstellten, wurde auf das laufende Monitoring großer Wert gelegt. Da Haus der Zukunft explizit als lernendes Programm konzipiert war, wurde für den Bereich der Leitprojekte zudem ein strategisches Monitoring durch einen externen Experten durchgeführt, um administrative und strategische Verbesserungsvorschläge strukturiert zu erheben und aufzubereiten. Die eingebrachten Vorschläge umfassten bspw. die Anregung, die Anzahl an Projekt- und Finanzierungsarten zu reduzieren oder die Auflagen der Bewertungsjury möglichst klar und konkret zu formulieren. Die Interviews im Zuge der vorliegenden Evaluierung schlossen inhaltlich an die Ergebnisse des strategischen Monitorings an. 
Im Rahmen eines zweistufigen Verfahrens wurden 8 Leitprojekte ausgewählt. Diese Leitprojekte umfassten insgesamt 60 Subprojekte, die zu Einreichstichtagen der ständigen Jury vorgelegt werden konnten. Für das Leitprojektmanagement wurden jeweils ca. $€ 40.000$ pro Jahr für max. drei Jahre vorgesehen. Die Anzahl der Subprojekte reichte von 3 bis 11 Projekten. 211 Projektpartner waren an den Leitprojekten sowie den damit verbundenen Subprojekten beteiligt.

Die beantragten Gesamtkosten beliefen sich auf gut $€ 51$ Mio., davon wurde knapp die Hälfte als Förderung beantragt. Genehmigt wurden Gesamtkosten in Höhe von $€ 34,7$ Mio. und eine Förderung von $€ 15,3$ Mio. Im Schnitt ergibt das Kosten von $€ 4,4$ Mio. und eine Förderung von $€ 1,9$ Mio. für jedes Leitprojekt.

Die nachfolgende Tabelle gibt einen allgemeinen Überblick über die umgesetzten Leitprojekte:

Tabelle 3: $\quad$ Übersicht Leitprojekte

\begin{tabular}{|c|c|c|c|c|}
\hline Titel & Projektleitung & Ort & $\begin{array}{l}\text { Subpro- } \\
\text { jekte }\end{array}$ & Thema \\
\hline $\begin{array}{l}\text { Stadtumbau } \\
\text { Lehen }\end{array}$ & $\begin{array}{l}\text { SIR Salzburger Institut } \\
\text { für Raumordnung } \& \\
\text { Wohnen }\end{array}$ & $\begin{array}{l}\text { Salz- } \\
\text { burg }\end{array}$ & 8 & $\begin{array}{l}\text { Siedlung / } \\
\text { Neubau / Sa- } \\
\text { nierung }\end{array}$ \\
\hline aspern plus & $\begin{array}{l}\text { Wien } 3420 \text { Aspern Deve- } \\
\text { lopment AG }\end{array}$ & Wien & 9 & $\begin{array}{l}\text { Siedlung / } \\
\text { Neubau }\end{array}$ \\
\hline $\begin{array}{l}\text { e80^3- } \\
\text { Gebäude }\end{array}$ & $\begin{array}{l}\text { AEE Intec - Institut für } \\
\text { Nachhaltige Technologien }\end{array}$ & $\begin{array}{l}\text { Gleis- } \\
\text { dorf }\end{array}$ & 6 & $\begin{array}{l}\text { Sanierung } \\
\text { (Fassadenmo- } \\
\text { dule) }\end{array}$ \\
\hline $\begin{array}{l}\text { Leuchtturm } \\
\text { Gugler }\end{array}$ & $\begin{array}{l}\text { pos architekten } \\
\text { ZT-KG }\end{array}$ & Wien & 9 & $\begin{array}{l}\text { Betriebsgebäu- } \\
\text { de / Neubau }\end{array}$ \\
\hline BI GMODERN & $\begin{array}{l}\text { Bundesimmobiliengesell- } \\
\text { schaft m.b.H. }\end{array}$ & Wien & $\begin{array}{l}10 \\
\text { (2 Demo) }\end{array}$ & $\begin{array}{l}\text { Sanierung Öf- } \\
\text { fentliche Ge- } \\
\text { bäude }\end{array}$ \\
\hline $\begin{array}{l}\text { GdZ - Grün- } \\
\text { derzeit mit } \\
\text { Zukunft }\end{array}$ & $\begin{array}{l}\text { e7 Energie Markt Analyse } \\
\text { GmbH }\end{array}$ & Wien & $\begin{array}{l}11 \\
\text { (4 Demo) }\end{array}$ & Sanierung \\
\hline $\begin{array}{l}\text { Plus-Energie- } \\
\text { Bürobau der } \\
\text { Zukunft }\end{array}$ & Schöberl \& Pöll GmbH & Wien & 4 & $\begin{array}{l}\text { Sanierung / } \\
\text { Bürogebäude }\end{array}$ \\
\hline $\begin{array}{l}\text { ECR Energy } \\
\text { City Graz Re- } \\
\text { ininghaus }\end{array}$ & $\begin{array}{l}\text { TU Graz, Institut für } \\
\text { Städtebau }\end{array}$ & Graz & 3 & $\begin{array}{l}\text { Siedlung / } \\
\text { Neubau }\end{array}$ \\
\hline
\end{tabular}

Quelle: Eigene Darstellung 
Das komplexe Zusammenspiel von Leitprojekten, Subprojekten und verbundenen Demonstrationsprojekten visualisiert die nachfolgende Grafik:

Abbildung 22: Leitprojekte, Subprojekte und verbundene Demonstrationsprojekte
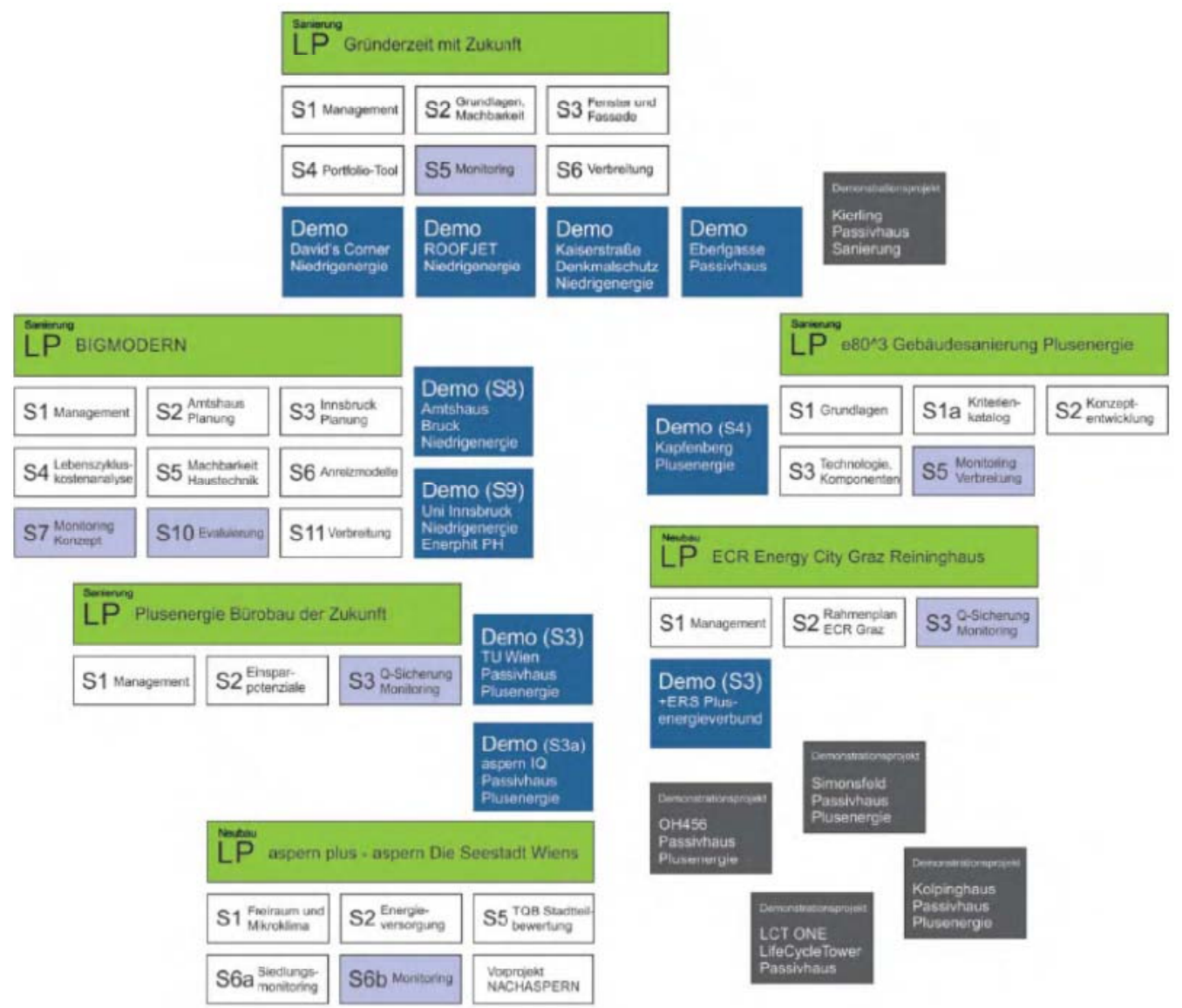

LP: Leitprojekt S: Subprojekt eines Leitprojekts Demo: Demonstrationsprojekt eines Leitprojekts

Quelle: Österreichisches Ökologie-Institut / Österreichisches Institut für Bauen und Ökologie 2015, 93

Die acht Leitprojekte wurden im Rahmen der Evaluierung einer detaillierten Analyse unterzogen. Dabei wurden sowohl die Umsetzung der Leitprojekte wie auch die damit erzielten Wirkungen fokussiert. Die nachfolgende Darstellung der Leitprojekte basiert im Wesentlichen auf entsprechenden Dokumenten und Interviews mit den Leitprojektmanagern. Eine Liste der Gesprächspartner sowie der Gesprächsleitfaden sind im Anhang zum Evaluierungsbericht angeführt. 


\subsubsection{Stadtumbau Lehen}

Der Stadtteil Lehen inmitten der Stadt Salzburg zeichnet sich durch seine Zentrumsnähe aus, stand jedoch vor großen strukturellen und sozialen Herausforderungen (hohe Verkehrsbelastung, Geschäftsschließungen, Überalterung, soziale/ethnische Spannungen etc.). Die Stadtverwaltung hat in dieser Krise die Chance gesehen, ein größeres Stadtentwicklungsgebiet nach städtebaulichen, ökologischen und sozialen Maßstäben zu revitaliseren und eine hohe Wohn- und Lebensqualität zu realisieren.

Dazu wurde auf mehreren Ebenen angesetzt: Im CONCERTO-Programm der Europäischen Kommission, das zum Ziel hat, die Energieeffizienz und den Anteil erneuerbarer Energien in ganzen Stadtteilen durch umfassende Konzepte und Umsetzungsprojekte zu erhöhen, konnte eine Beteiligung am Projekt "Green Solar Cities" erreicht werden. Im Zeitraum 2007-2009 wurden einige Großprojekte zum nachhaltigen Umbau Lehens über dieses EU-Programm realisiert. Allein am ehemaligen Areal des Stadtwerks Lehen mit $43.000 \mathrm{~m}^{2}$ entstand eine Anlage mit 300 Wohnungen, ein Kindergarten und Studentenheim, darüber hinaus ein Seniorenwohnhaus sowie weitere Wohn- und Bürohäuser - durchwegs in Niedrigstenergiehaus-Standard. Das Land Salzburg hat sich im Rahmen der Wohnbauförderung ebenfalls stark in diesem Vorhaben engagiert.

Die Ausschreibung zu Haus der Zukunft-Leitprojekten erwies sich zeitlich und thematisch als wertvolle Ergänzung dazu. Dem Programmziel entsprechend konnten im Rahmen des Leitprojektes insbesondere jene Vorhaben realisiert werden, die auf die gesamthafte Entwicklung des Stadtteils abzielten, sowie - in ihrer Bedeutung nicht zu unterschätzende - spezifische Begleitmaßnahmen mit vorrangig sozialwissenschaftlichen Inhalt.

Folgende Projektpartner waren am Leitprojekt beteiligt:

* SIR - Salzburger Institut für Raumordnung \& Wohnen (Leadpartner)

* Stadt Salzburg / Amt für Stadtplanung

* gswb - Gemeinnützige Wohn- und Siedlungsgesellschaft

* Salzburg AG für Energie, Verkehr und Telekommunikation

* Die "Salzburg“ - Gemeinnützige Wohn- und Siedlungsgenossenschaft

* PRISMA Zentrum für Standort- und Stadtentwicklung GmbH

* Steinbeis-Transferzentrum Energie-, Gebäude- und Solartechnik (Stuttgart)

* wohnbund:consult - Büro für Stadt. Raum. Entwicklung 
Ziel des Leitprojekts war die Erreichung eines möglichst großen Anteils von erneuerbaren Energien im gesamten Stadtteil. Nachhaltigkeit wurde dabei in einem breiten Ansatz verstanden und beinhaltete neben dem ökologischen Neubau auch die Sanierung, Mobilität, Freiraumplanung, soziale Aspekte etc. Der gesamte Stadtteil wird mit Fernwärme versorgt, welche einen Anteil von ca. 40 - $45 \%$ an erneuerbaren Energien aufweist. Eine thermische Großsolaranlage mit $2.000 \mathrm{~m}^{2}$ Kollektor mit einer verbesserten Puffertechnologie und einem lokalen Mikronetz ermöglichte eine "Veredelung" der Fernwärme, sodass in Summe ein Anteil an erneuerbaren Energien von ca. $65 \%$ erreicht wurde.

Ein zentrales Thema des Leitprojektes war das Energiemonitoring. Den Bewohnern sollte der Energieverbrauch der Gebäude aufgezeigt werden, um Bewusstsein, Motivation für und Akzeptanz von nachhaltigen Energiekonzepten zu schaffen. Hierzu wurden unterschiedliche Modelle und Ansätze ausprobiert, etwa ein intelligentes e-Monitoring: Informationsmonitore beim Hauseingang visualisierten den aktuellen Energieverbrauch, die Energiekosten pro Wohnung und den aktuellen Energiemix anhand einer "Energieampel", die bei hohem Verbrauch auf rot schaltete und bei geringem Verbrauch grün anzeigte. Es stellte sich heraus, dass die Bewohner aus verschiedenen Gründen schwer zu motivieren sind bspw. die Raumtemperatur abzusenken. Möglicherweise spielt auch der Umstand eine Rolle, dass die Energiekosten des Niedrigstenergiehauses im Schnitt $30 \%$ unter den üblichen Kosten liegen und so der Eindruck entsteht, ein besonders sparsames Verhalten wäre nicht erforderlich („Rebound-Effekt“).

Ebenfalls eine soziale Dimension haben die übergreifende Grünraum- und Mobilitätsplanung. Die Grünraumplanung erfolgte mit Blick auf den gesamten Stadtteil, auch wurde erstmals in Salzburg ein grundstücksübergreifendes Radwegenetz realisiert.

Die Neubauphase ist zum jetzigen Zeitpunkt abgeschlossen, allerdings ohne Titulierung als Haus der Zukunft-Demonstrationsprojekte. Das bereits erwähnte Energiemonitoring soll weiter fortgesetzt werden - in sozialwissenschaftlicher Hinsicht haben sich noch Forschungsfragen gezeigt, die im Rahmen des Projektes nicht befriedigend beantwortet werden konnten. Darüber hinaus wird in den nächsten Jahren noch die Sanierung von 800 Altbauwohnungen in mehreren Etappen vorgenommen.

Die Ergebnisse des Projektes Stadtumbau Lehen werden nun laufend verbreitet. Durch die intensive Zusammenarbeit mit europäischen Partnern ist eine hohe überregionale Sichtbarkeit gegeben. In wirtschaftlicher Hinsicht ergaben sich jedoch keine unmittelbaren Verwertungsmöglichkeiten, da die beteiligten Unternehmen dem gemeinnützigen Wohnbau angehören. 


\subsection{2 aspern plus}

Die nachhaltige Ausrichtung eines gesamten Stadtteils stand auch im Mittelpunkt des Leitprojekts „aspern plus“. In der Seestadt Aspern im 22. Wiener Gemeindebezirk wird auf 240 Hektar das größte Stadtentwicklungsprojekt Österreichs umgesetzt. In den nächsten 20 Jahren entsteht hier ein neuer Stadtteil, der 20.000 Arbeitsplätze und ebenso viele Wohnungen umfassen wird. Dabei wird auf eine zukunftsweisende, innovative und energieoptimierte Stadt- und Gebäudeplanung großer Wert gelegt. Die "Wien 3420 Aspern development AG" hat das gesamte Projektmanagement in diesem Vorhaben inne.

Im Rahmen des Haus der Zukunft-Forschungsprojektes NACHASPERN wurden die zentralen Kriterien und Maßnahmen entwickelt, die bei der Vergabe der Baufelder als Grundlage für eine nachhaltige Entwicklung dienen. Ein wichtiger Aspekt war dabei die Betrachtung des Energiebedarfs - sowohl in Hinblick auf die einzelnen Gebäude wie auch den gesamten Stadtteil. Hierfür wurde ein Gesamtenergieplan erstellt und die definierten Kriterien in allen Vergabeprozessen berücksichtigt (Architekturwettbewerbe, Bauträgerwettbewerbe, Direktvergabe).

Auf diese Vorarbeiten konnte im Leitprojekt „aspern plus“ unmittelbar angeknüpft werden. Die „Wien 3420 Aspern Development AG" fungierte dabei als Konsortialführer, weiters waren verschiedene wissenschaftliche Einrichtungen (diverse Institute der Technischen Universität Wien, Austrian Institute of Technology, Österreichisches Ökologie-Institut, research Tub) beteiligt.

Ziel des Projekts war es, die bestehenden Vorarbeiten auszuweiten und zu vertiefen. Dabei sollten etwa die Themen Freiraumplanung, gebäudeübergreifendes Energiesystem und Energieverbrauchsmonitoring behandelt werden. Auf dieser Grundlage sollten Demonstrationsprojekte im Plusenergie-Standard errichtet werden. Daneben sollte die gesamte Siedlungsentwicklung in energetischer, ökologischer und sozialer Hinsicht nachhaltig gestaltet werden.

Analog dazu wurden sechs Subprojekte umgesetzt:

1. Freiraum und Mikroklima: Untersuchung des Zusammenhangs zwischen Freiräumen und Mikroklima im Rahmen der Stadtteilentwicklung

2. Gebäudeübergreifende Energie: Entwicklung eines Tools zur Analyse und Prognose des Energieaustauschpotenzials zwischen Gebäuden

3. Demonstrationsgebäude: Fertigstellung des Innovationszentrums „aspernIQ“ im Herbst 2012

4. Investive Maßnnahmen Mobilität: Multimodales Mobilitätsmanagement (oberund unterirdische Parkmöglichkeiten, Anbindung der Siedlung an den öffentlichen Verkehr, Radwege und - abstellmöglichkeiten) 
5. Qualitätsmonitoring: Orientierung an Total Quality Building-Standards von der Planung bis zur Abnahme der Gebäude

6. Energieverbrauchsmonitoring: Energieverbrauchsmonitoring für das Demonstrationsgebäude sowie die gesamte Siedlung

Die ursprünglich vorgesehene Errichtung eines Wohngebäudes und eines Studentenheims als weitere Demonstrationsgebäude zeigten sich als nicht realisierbar. Für das Wohngebäude war vorgesehen, die lokal erzeugte Energie zu nutzen und einen allfälligen Energieüberschuss in das öffentliche Netz einzuspeisen. Diese Überschusseinspeisung ist nach derzeitiger Rechtslage bei Mehrparteienhäusern nicht möglich, da sie künftigen Mietern/Eigentümern die freie Wahl des Stromanbieters unterbinden würde. Für das Studentenheim waren die technischen Auflagen aus Sicht des Betreibers zu hoch. Weiters war die Errichtung eines Geothermiekraftwerks geplant, wovon nach Probebohrungen Abstand genommen wurde.

Eine zentrale Erkenntnis aus dem Leitprojekt "aspern plus“ ist, dass Plusenergiestandard in einem Gebäude und in einer Siedlung realisierbar sind und mit einem hohen Nutzungskomfort einhergehen. Dies kann allerdings nur erreicht werden, wenn alle Planungen und Bauausführungen konsequent darauf ausgerichtet sind. Auch die Gebäudenutzung spielt dabei eine wichtige Rolle: So ist es bei einem Büro- oder Wohngebäude mit unterschiedlichen Eigentümern und Nutzern kaum möglich, alle Beteiligte zur Verwendung energieoptimierter Geräte zu animieren. Ferner ist bspw. im Technologiezentrum aspernlQ die Firmen "researchTUb" - eine Ausgründung der Technischen Universität Wien - ansässig, die dort in einer Pilotfabrik innovative Produktionsprozesse erforscht. Damit geht ein höherer Energieverbrauch einher als etwa bei einem reinen Dienstleistungsbetrieb.

Damit offenbart "aspern plus" eine wichtige Frage in Bezug auf Nachhaltiges Bauen. Nachhaltigkeit und Energieoptimierung sind zweifelsohne erstrebenswerte Ziele, stehen zuweilen aber im Konflikt mit anderen Interessen. Dazu zählen etwa die Ansiedlung von produzierenden Unternehmen auch im urbanen Umfeld, die Vorgaben in Bezug auf die Baukosten im sozialen Wohnbau, das Prinzip der Freiheit in der Wahl von Energieanbietern oder energieverbrauchenden Geräten uvam. Um zu einer höheren Durchdringung nachhaltiger Bauformen zu kommen, wird es unausweichlich sein, die verschiedenen umwelt-, sozial- und wirtschaftspolitischen Aspekte der Stadt- und Raumplanung abzuwägen.

Die Wirkung des Leitprojektes begründet sich insbesonders durch den Pilotcharakter des gesamten Stadtentwicklungsprojektes eine hohe Dimension. Die Seestadt Aspern hat innerhalb und außerhalb Österreichs eine hohe Sichtbarkeit, wobei auch die im Leitprojekt gewonnenen Erkenntnisse und das realisierte Demonstrationsgebäude eine große Resonanz erfahren. Internationale Delegationen besuchen laufend die Seestadt und das Technologiezentrum aspernlQ. Vice versa wird das Projekt laufend auf Konferenzen, Fachmessen u.dgl. vorgestellt. 


\subsection{3 $\mathbf{e 8 0 \wedge 3}$}

Die Fassade von Gebäuden macht einen großen Teil der Außenhülle aus - dennoch wird sie meist nur für Dämmzwecke genutzt. Der Gedanke, Dach- und Fassadensysteme vorzufertigen und mit mehr Funktionen auszustatten, liegt daher nahe. Die steirische Forschungseinrichtung AEE Intec hat sich u.a. im Rahmen des internationalen Forschungsprojektes „IEA ECBCS Annex 50 - Prefabricated Systems for Low Energy Renovation of Residential Buildings" (2007-2010) gemeinsam mit anderen europäischen Partnern damit befasst.

Auf diesen internationalen Erkenntnissen aufbauend wurde das Leitprojekt „e80^3“ konzipiert, welches diesen Ansatz von der Theorie bis hin zur praktischen Realisierung und anschließend Evaluierung verfolgte. Kerngedanke des Projektes war es, die Energieeffizienzpotenziale von zwischen 1950 und 1980 errichteten Gebäuden im Rahmen einer Sanierung zu erschließen. Hierbei kamen vorgefertigte aktive Dach- und Fassadenelemente mit integrierter Haustechnik und Netzintegration zum Einsatz.

Das Sanierungskonzept beruhte auf Effizienzmaßnahmen (hoch gedämmte, vorgefertigte aktive Energiedach- und Energiefassadenelemente mit integrierter Haustechnik), auf einem hohen Anteil an erneuerbaren Energien sowie auf einer intelligenten Integration der Energieversorgung in Wärme- und Stromnetze. Dabei trug das Konzept signifikant zur Erreichung der drei maßgeblichen EU-Ziele bei aus diesen leitet sich auch der Projekttitel ab:

* $80 \%$ Energieeffizienz - Reduktion des Energiebedarfs

* 80 \% Anteil erneuerbarer Energie am Gesamtenergieverbrauch

* $80 \%$ Reduktion der $\mathrm{CO}_{2}$-Emissionen

Das Leitprojektmanagement hatte AEE Intec - Institut für nachhaltige Technologien inne, eine außeruniversitäre Forschungseinrichtung in den Bereichen thermische Solarenergienutzung, Entwicklung von Niedrigenergie- und Nullenergiegebäuden und deren effiziente Energieversorgungssysteme sowie Energieeffizienz in der Industrie. Als weitere Partner waren die folgenden Einrichtungen involviert:

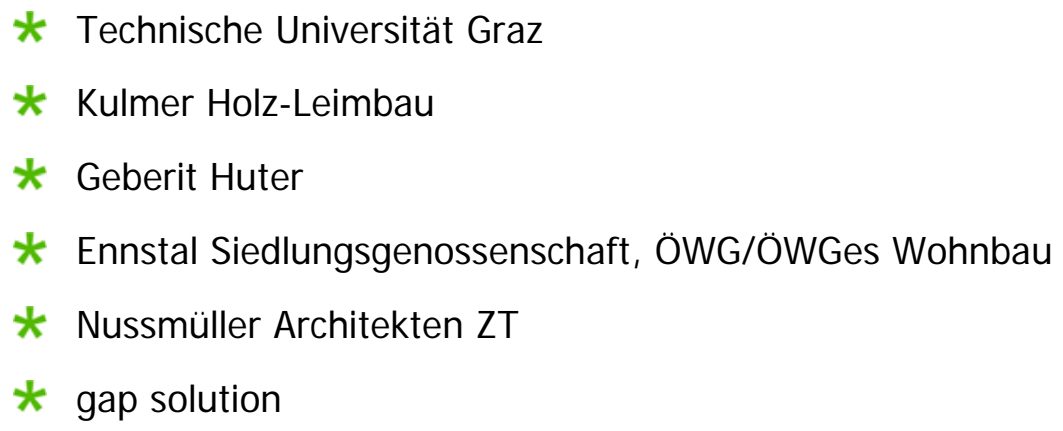




\section{GreenOne Tech}

* Stadtwerke Kapfenberg, Feistritzwerke Gleisdorf

Entsprechend der Intention der Leitprojekte, einen innovativen Ansatz von der Idee bis zur Realisierung und Verbreitung konsequent zu verfolgen, wurde das Leitprojekt konzipiert. In einem ersten Schritt wurden Grundlagenarbeiten durchgeführt, um auf dieser Basis ein innovatives und integriertes Sanierungskonzept entwickeln. Durch die hochwertige thermische Sanierung der Außenhülle mit Passivhauskomponenten und einer gleichzeitigen Integration von energieerzeugenden Aktivelementen wie thermischen Kollektoren oder Photovoltaik und einer Netzintegration für Strom und Wärme als Speicher- und Verteilfunktion sollte in der Sanierung von älteren Gebäuden ein Plus-Energiestandard erreicht werden.

Im dritten Subprojekt wurde die Technologie- und Komponentenentwicklung von Prototypen für vorgefertigte Fassadenelemente und Ver- und Entsorgungssysteme vorgenommen. Besonders die hochbautechnischen und bauphysikalischen Aspekte wurden dabei betrachtet und in die Überlegungen miteinbezogen. Auch wurden bereits realisierte innovative Haus der Zukunft-Projekte im Detail analysiert und die dabei gewonnenen Erkenntnisse einbezogen.

Für die Realisierung im Rahmen eines Demonstrationsgebäudes konnte in Kapfenberg ein geeignetes Gebäude gefunden werden. Die vorhergehenden Entwicklungen im Bereich der aktiven und passiven Fassadenmodule und Haustechnikmodule wurde dabei unmittelbar in der Praxis erprobt.

Ein Schwerpunkt wurde weiters auf das laufende Monitoring und die Verbreitung der Ergebnisse gelegt. Rund 15-20 nationale und internationale Delegationen haben seit Beginn der Sanierung im Jahr 2012 das Projekt besucht. Ferner wurde das Projekt im Rahmen von Konferenzen und Fachmessen vorgestellt und in der Scientific Community sichtbar gemacht. In der Folge hat sich auch wiederum ein internationales Projekt zu diesem Themenfeld ergeben, an dem die AEE Intec beteiligt ist (IEA EBC Annex 56 "Cost Effective Energy and Carbon Emissions Optimization in Building Renovation").

Die zentrale Erkenntnis des Projektes „e80^3“ liegt darin, dass vorgefertigte Dach- und Fassadenelemente mit integrierter Haustechnik ein vielversprechender Ansatz sind. Um eine Reduktion des Energieverbrauches im Baubereich zu erreichen, braucht es innovative Ansätze zur nachhaltigen Sanierung. Die Fassade ist dabei ein wichtiger Schlüssel, wobei durch die industrielle Vorfertigung von Elementen Kosteneinsparungen erzielt werden können. Derzeit sind die Mehrinvestitionen gegenüber konventionellen Technologien allerdings noch zu hoch, um eine breite Anwendung ohne öffentliche Förderung zu ermöglich. Daher wird auf den Aspekt der Kostenreduktion bei weiteren Forschungen und Entwicklungen hohes Augenmerk zu legen sein. 


\subsubsection{I nnovationsleuchtturm gugler cross media}

Nachhaltiges Bauen beinhaltet viele Aspekte. Ein Alleinstellungsmerkmal von Haus der Zukunft war es, das Thema in seiner gesamten Breite anzusprechen. Das Leitprojekt „I nnovationsleuchttrum gugler cross media“ macht diesen ganzheitlichen Zugang zur Thematik - aber auch die Hindernisse, denen Nachhaltiges Bauen in der Praxis gegenübersteht - besonders deutlich.

Die Leitprojektmanagerin Arch. DI Ursula Schneider hat sich besondere Expertise in der Planung und Ausführung nachhaltiger Gebäude erworben, insbesondere durch zahlreiche Haus der Zukunft-Projekte. Bei der Besichtung eines besonders energieeffizienten Bürogebäudes entstand ein Kontakt zum Eigentümer des niederösterreichischen Mediendienstleistungsunternehmens Gugler, der eine Adaptierung und Erweiterung des Betriebsgebäudes nach besonders nachhaltigen Kriterien beabsichtigte. Da die Planungsphase in den Zeitraum der Ausschreibung für Haus der Zukunft-Leitprojekte fiel, bot sich die Einreichung unmittelbar an.

Ziel des Leitprojekts "Innovationsleuchtturm gugler cross media“ war es, eine neue Dimension der Nachhaltigkeit im Bauen zu erreichen. Dieses neue Level besteht darin, nicht nur in energetischer Hinsicht hohe Ziele zu verfolgen (Energieeffizienz, Einsatz erneuerbarer Energien), sondern auch in stofflicher Hinsicht. Auf den Punkt gebracht lautet der Ansatz: „Zero energy, zero waste“. Das Gebäude sollte in einem Stoff- und Energiefluss gesehen werden, der über die Errichtung und den Betrieb des Gebäudes bis hin zum Abriss und Recycling der Baumaterialen den gesamten Lebenszyklus eines Gebäudes berücksichtigt.

Das Leitprojektmanagement hatte die pos architekten ZT KG inne, unterstützt von folgenden Projektpartern:

* Gugler GmbH - Cross Mediales Kommunikationshaus

* IBO - Österreichisches Institut für Baubiologie und -ökologie

* Alchemia nova - Institut für innovative Pflanzenforschung Wien

* New Energy Consulting - Ingenieurbüro für Neue Energie und Gebäudetechnik

Zunächst wurde im Rahmen von vorrangig theoretischen Subprojekten untersucht, inwieweit Recyclingmaterialen und nachwachsende Rohstoffe im Bau eines Büro- und Betriebsgebäudes eingesetzt werden können und worauf hierbei in der Konstruktion zu achten ist. Dabei wurde der gesamte Kreislauf von der Produktion der Baustoffe und Komponenten, der Bereitstellung von Energie bis hin zur Entsorung bzw. Wiederverwertbarkeit berücksichtigt. Es zeigte sich, dass gerade in Bezug auf die stoffliche Dimension Nachhaltigen Bauens bislang wenig Erfahrungswerte bestehen und so wissenschaftliches Neuland betreten wurde. 
Das Leitprojekt zeichnete sich durch einen sehr hohen I nnovationsgrad aus, der zum Einen in der Erforschung geeigneter Materialien, Komponenten und Planungsgrundlagen für ein Plusenergie-Betriebsgebäude mit ökologischer Kreislauffähigkeit begründet liegt. Zum Anderen wurden die dabei gewonnenen Erkenntnisse sogleich in ein konkretes Gesamtenergie- und -gebäudekonzept integriert und damit die praktische Umsetzung vorbereitet.

Als Subprojekt war die Adaption und Erweiterung eines Betriebsgebäudes der Firma Gugler von ca. $2.140 \mathrm{~m}^{2}$ auf ca. $5.400 \mathrm{~m}^{2}$ vorgesehen. Es ist offensichtlich, dass mit einer solchen Erweiterung hohe Investitionskosten verbunden sind, zumal in diesem Fall hohe energetische und stoffliche Maßstäbe zugrunde gelegt worden sind. Obgleich die Mehrkosten durch die Haus der Zukunft-Förderung reduziert worden wären, so war doch ein erheblicher Finanzierungsbedarf gegeben. Entgegen dem ursprünglichen Konzept konnte das Unternehmen keine Bank für eine Finanzierung dieses Projektes gewinnen. Derzeit werden der Demonstrationsbau in kleinerer Dimension neu geplant und alle Möglichkeiten einer Realisierung verfolgt.

So bedauerlich diese Entwicklung ist, sie zeigt doch eine wesentliche Erkenntnis des Leitprojektes auf: Nachhaltigkeit im Bau wird vielfach nur auf die Energie bezogen, beinhaltet aber noch weitere Aspekte. Nachhaltige Kriterien für Baumaterialien in Hinblick auf deren gesamten Lebenszyklus sind noch genauer zu entwickeln. Ferner bedarf es mehr Informationen über und Bewusstsein für die Gesamtlebenskosten eines Gebäudes - dies inkludiert auch die finanziellen und ökologischen Kosten für die Entsorgung der Baustoffe.

Höhere Investitionen in energiesparende Gebäude lassen sich leicht über Amortisationsrechnungen darstellen. Höhere Investitionen in nachhaltige Materialien lassen sich bislang nicht in dieser Form abbilden, da im laufenden Betrieb keine Kosteneinsparung eintritt, sondern erst bei der Entsorgung. Auch aus einer ökologischen Perspektive ist das Thema von hoher Relevanz: Energie ist in nahezu unendlichem Ausmaß vorhanden - etwa Sonnen-, Wasser- oder Windenergie. Nicht nachwachsende stoffliche Ressourcen sind hingegen endlich.

Die wirtschaftliche und wissenschaftliche Verwertung der Projektergebnisse basiert auf diesen Erkenntnissen. Dem Charakter eines Forschungsprogrammes entsprechend konnte im Rahmen dieses Haus der Zukunft-Leitprojektes neues Wissen generiert werden, das nun die Grundlage für konkrete freifinanzierte Nachfolgeprojekte sowie weitere Forschungsarbeiten darstellt. 


\subsubsection{BI GMODERN}

Die Bundesimmobiliengesellschaft (BIG) ist mit einem Anlagevermögen von ca. $€ 9$ Mrd. und 2.200 verwalteten Liegenschaften der größte Immobilieneigentümer Österreichs. Es ist offensichtlich, dass die BIG ein zentraler Akteur bei der Erzielung von Einsparungseffekten im Gebäudebereich in Österreich ist. Zudem sind öffentliche Einrichtungen durch die EU-Gebäuderichtlinie verpflichtet, bei Neubauten bis 2019 den "nearly zero emission“-Standard zu erreichen.

Vor diesem Hintergrund befasste sich die BIG intensiver mit dem Thema Nachhaltigkeit. Ein großer Hebel hierfür war die Sanierung von Gebäuden aus den 1950 er bis 1980er Jahren - diese Bauten werden nun laufend saniert und dabei bietet sich die Gelegenheit zur Energieeinsparung. Passfähige Kriterien und Standards, die für die BIG und ihre Auftraggeber handhabbar sind, fehlten dabei ebenso wie entsprechende Demonstrationsgebäude im Bereich der Sanierung.

Als sich zwei konkrete Projekte für ein solches Pilotvorhaben anboten, hat die BIG ein Konsortium gebildet und den Lead für ein Haus der Zukunft-Leitprojekt unter dem Titel „BIGMODERN“ übernommen. Folgende Einrichtungen fungierten als weitere Partner:
* e7 / Energie Markt Analyse
* GEA Graz Energy Agency
* Pittino \& Ortner
* Achhammer Tritthart \& Partner
* TU Innsbruck
* Bundesministerium für Finanzen
* Bundesministerium für Justiz
* Bundesamt für Eich- und Vermessungswesen

Ziel des Projektes war es, Nachhaltigkeits- und Klimaschutzkriterien für die Modernisierung von Bundesgebäuden der Nachkriegsperiode zu entwickeln. Diese Zielkriterien sollten im Rahmen von Demonstrationsprojekten auf ihre Praxistauglichkeit hin überprüft werden und in weiterer Folge als wesentliche Leitprinzipien in den Planungs- und Ausführungsprozessen für sämtliche zukünftige Modernisierungsvorhaben der BIG definiert werden.

Angesichts des hohen Anteils von Modernisierungsvorhaben an den Gesamtinvestitionen der BIG sowie der großen Optimierungspotenziale im Zuge einer Sanierung sind gerade in diesem Bereich besonders innovative Lösungen erforderlich, die zudem den Kriterien der Wirtschaftlichkeit entsprechen. Das Leitprojekt hat daher in einem ersten Schritt die konkreten Hürden und Barrieren identifiziert und 
eschrieben, die sich bei der nachhaltigen Sanierung öffentlicher Gebäude eröffnen. Dazu zählen u.a. die mit innovativen Lösungen verbundenen Risken für die Bauherrn und Planer, die Berücksichtigung von Nachhaltigkeits- und Energieeffizienzkriterien bereits zu einem frühen Zeitpunkt im Planungsprozess und die Wirtschaftlichkeitsrechnung.

In einem zweiten Schritt wurde in zwei großen Demonstrationsprojekten die praktische Umsetzung erprobt und ein Amtshaus in Bruck an der Mur aus dem Jahr 1965 sowie das Gebäude der Fakultät für Bauingenieurwissenschaften an der Technischen Universität Innsbruck aus dem Jahr 1971 saniert. Die Bauten haben jeweils eine Nettogrundfläche von ca. $6.500 \mathrm{~m}^{2}$ und die Sanierungskosten betrugen jeweils ca. $€ 8$ Mio (netto). Die sanierten Gebäude erfahren nun eine große Resonanz und werden laufend von nationalen und internationalen Delegationen besucht.

In einem begleitenden Forschungsteil wurden die für die Umsetzung der Demonstrationsprojekte erforderlichen Entscheidungen wissenschaftlich unterstützt. So wurden bspw. planungsbegleitende Lebenszykluskostenanalysen durchgeführt.

Die Demostrationsprojekte haben eine besondere Vorbildwirkung für öffentliche und private Bauträger, Bauherrn und Nutzergruppen erzielt. Innovative und nachhaltige Standards bei Modernisierungsvorhaben sind mit langfristig geringen Mehrkosten realisierbar. Es hat sich aber auch gezeigt, dass bei vielen Beteiligten an einem derartigen Vorhaben Wissensgrundlagen vermittelt und realistische Erwartungen geweckt werden müssen. Die Kosten spielen gerade im öffentlichen Bereich eine große Rolle - hier sind neue Modelle der Kostendarstellung aus einer langfristigen Perspektive erforderlich.

Die Erfahrungen und das Know-how, welche durch die Planung und Bauausführung der beiden Demonstrationsprojekte generiert wurden, bildeten die fachliche Grundlage für die Formulierung allgemeiner Leitprinzipien für die nachhaltige Sanierung öffentlicher Gebäude. Diese Standards wurden in weiterer Folge im Rahmen eines „Holistic Building Program“ der BIG aufbereitet. Ein wichtiges Anliegen ist hierbei, Nachhaltigkeitskriterien nicht nur aus ökologischen Motiven zu berücksichtigen, sodern in erster Linie eine Steigerung der Gebäudeattraktivität und des Nutzungskomforts zu erzielen.

Das Leitprojekt hat damit wesentlich dazu beigetragen, das Wissen über die Hürden und Potenziale der energieeffizienten Sanierung öffentlicher Gebäude zu vertiefen und anhand von Demonstrationsprojekten zu veranschaulichen. Die schrittweise Verankerung von daraus abgeleiteten Prinzipien wird wesentlich dazu betragen, die energetischen Anforderungen an öffentliche Gebäude zu erreichen. 


\subsubsection{Gründerzeit mit Zukunft}

Neben dem Neubau kristallisierte sich die Sanierung von Bestandsobjekten als großes Innovationsfeld, Wachstumsmarkt und Hebel, um den Energieverbrauch in Gebäuden dauerhaft zu senken, als zentrale thematische Säule von Haus der Zukunft heraus. Österreichweit existieren mehr als 600.000 Wohnungen in Gebäuden aus der Gründerzeit - knapp ein Fünftel des gesamten Wohnungsbestandes in Österreich wurde vor 1919 errichtet. Allein in Wien finden sich rund 20.000 klassische Gründerzeit-Zinshäuser, das entspricht rund $30 \%$ aller HauptwohnsitzWohnungen in der Bundeshauptstadt.

Das Leitprojekt „Gründerzeit mit Zukunft“ hat sich zum Ziel gesetzt, Häuser aus dieser Bauperiode unter Anwendung innovativer technischer und organisatorischer Lösungen zu modernisieren. Damit sollte die thermisch-energetische Qualität der Bauten verbessert und ihr einzigartiger Charakter erhalten werden, denn Gründerzeithäuse stellen eine wichtige Säule des baukulturellen Erbes in Österreich dar.

Die Projektleitung lag beim Büro „e7 Energie Markt Analyse GmbH“. Ein interdisziplinäres Team bildeten die weiteren Projektpartner:

* Havel \& Havel Beratungs GmbH (Sozialwissenschaft)

* Manschein Managing Energy (Monitoring)

* Österreichischer Verband der Immobilientreuhänder (Wohnrecht)

* pos architekten ZT (Architektur)

* Schöberl \& Pöll OEG (Bauphysik)

* Gemeinschaft Dämmstoff Industrie (Dissemination)

* Partner in den Demonstrationsprojekten (Eigentümer/Hausverwaltung)

In einem ersten Schritt wurden Konzepte entwickelt, die innovative Technologien aus dem Passivhausbau auf die Spezifika von Gründerzeitbauten adaptierten und organisatorische Lösungen für die bauliche Umsetzung beinhalteten.

Diese wurden anschließend in vier Demonstratationsgebäuden zur Anwendung gebracht, die allesamt in Wien lokalisiert sind:

* In der Wißgrillgasse in Penzing wurden wärmetechnische Schwachstellen gezielt eliminiert, eine Außendämmung vorgenommen und die Fenster erneuert. Die Fassade wurde originalgetreu nachgebildet. Ein Dachgeschoßausbau und eine Gebäudeumstrukturierung brachten zusätzliche Wohnfläche, wodurch die Gesamtkostenrechnung attraktiver gestaltet werden konnte. Der Heizwärmebedarf wurde so von $185 \mathrm{kWh} / \mathrm{m}^{2}$ a auf $27,5 \mathrm{kWh} / \mathrm{m}^{2}$ a bzw. Niedrigstenergiestandard gesenkt. 
Das Projekt Kaiserstraße in Neubau stellte eine besondere Herausforderung dar, da es sich in ein historisches Ensemble einfügt und unter Denkmalschutz steht. Änderungen am äußeren Erscheinungsbild waren daher nur in geringfügigem Ausmaß möglich. Dennoch fanden sich viele Optimierungsmöglichkeiten, etwa durch eine Innendämmung, innenliegende Fenster oder eine zentrale Lüftungsanlage mit Wärmerückgewinnung. Somit wurde der rechnerische Heizwärmebedarf um rund $80 \%$ gesenkt.

* Ein Gebäude in der Eberlgasse (Leopoldstadt) konnte im Zuge einer grundlegenden Sanierung auf Passivhausstandard adaptiert werden. Die Umbauten erfolgten in Kooperation mit allen Mietparteien und konnten ohne Ausmietung realisiert werden. Einsparungen von über 90 \% bezogen auf den Heizwärmebedarf, den Endenergiebedarf und die $\mathrm{CO}_{2}$-Emissionen sind zu erwarten.

* Derzeit befindet sich das vierte Projekt "David's Corner" in Favoriten in der Finalisierung. Drei miteinander verbundene Gebäude werden dabei in energetischer und wohnstandardlicher Hinsicht modernisiert, u.a. durch DachgeschoBausbauten, Wärmedämmung und Erneuerung der Fenster. Eine Besonderheit bildet das gemeinschaftliche Haustechnikkonzept für alle drei Gebäude unter Einbeziehung von Fernwärme, Solarenergie und Wohnraumlüftung.

Die Realisierung der Demonstrationsgebäude brachte einige Herausforderungen mit sich. Es liegt in der Natur sowohl von Forschungsprojekten wie auch von Bauvorhaben, dass es zu Abweichungen von der ursprünglichen Planung kommen kann. Dies wurde bei den ambitionierten Vorhaben dieses Leitprojektes deutlich, sodass von den beantragten fünf Demonstrationsgebäuden nur drei umgesetzt werden konnten, jedoch ein weiteres hinzukam. Die Realisierung mehrerer eigenständiger Bauprojekte mit unterschiedlichen Eigentümern war zudem kein einfaches Unterfangen, sodass es zu erheblichen zeitlichen Verzögerungen kam. Letzten Endes werden rund 10 Jahre von der ersten Idee bis zum Abschluss des Monitorings von Demonstrationsgebäuden vergangen sein.

Die wesentliche Erkenntnis von "Gründerzeit mit Zukunft" ist, dass eine energieoptimierte Sanierung von historischen Gebäuden, sogar von jenen unter Denkmalschutz, mit vertretbaren Mehrkosten möglich ist. Der Schlüssel liegt in der individuellen Herangehensweise: Die Ausgangssituation muss im Detail analysiert werden, damit Experten der verschiedenen Disziplinen und Gewerke passfähige Lösungen entwickeln können. Zudem steigert eine solche Sanierung auch den Wohnkomfort und die Behaglichkeit, sodass historische Gebäude ein sehr attraktives Wohnumfeld darstellen können.

Wenngleich der Ansatz, mehrere Gebäude als Demonstrationsprojekte im Rahmen eines Leitprojektes zu sanieren, seine Herausforderungen mit sich bringt, so liegt in diesem Umstand auch ein großer Beitrag zur Wirkung des Projektes. Auf diesem Weg wird offensichtlich, dass die Technologien und Lösungsansätze nicht nur 
bei einem Leuchtturmprojekt anwendbar sind, sondern für den breiten Einsatz tauglich sind. Allerdings ist dafür viel spezifisches Know-how erforderlich. Gerade dieses findet beim Leitprojekt große Beachtung, auch von internationalen Delegationen aus aller Welt (zB Brasilien). Aufgrund des Projektes "Gründerzeit mit Zukunft" wurde weiters dem mit der Projektleitung betrauten Unternehmen die Mitwirkung an einem Horizon 2020-Projekt ermöglicht. Ferner wurde das Projekt Eberlgasse mit dem "ETHOUSE-Award 2014" in der Kategorie Mehrfamilienhaus ausgezeichnet.

\subsubsection{Plus-Energie-Bürobau der Zukunft}

Trotz der enormen Entwicklungen im Bereich des energieeffizienten Bauens gibt es noch große Potenziale zur Energieoptimierung von Bürogebäuden. Ein Haus der Zukunft-Leitprojekt hat sich explizit diesem Thema gewidmet und sich zum Ziel gesetzt, den „Bürobau der Zukunft“ zu konkretisieren und zu realisieren. Im Mittelpunkt der Betrachtung standen dabei die technische Konzeption und Detaillierung eines Plus-Energie-Büros unter Berücksichtigung der rechtlichen und wirtschaftlichen Marktbedingungen.

Ziel des Projektes war es, den gesamten Energieverbrauch eines Gebäudes sowohl in der Errichtungs- wie auch der Nutzungsphase - soweit zu reduzieren und durch erneuerbare Energien zu unterstützen, dass das Gebäude mehr Energie erzeugt als es verbraucht (Plus-Energie-Standard). Im Rahmen der Projektvorbereitung wurden ähnliche Projekte weltweit analysiert und festgestellt, dass es in dieser umfassenden Betrachtung und auf derart hohem energetischem Standard kein vergleichbares Gebäude gibt.

Das Leitprojektmanagement hatte das Wiener Bauphysikbüro Schöberl \& Pöll inne. Eigentümerin der Liegenschaft ist die Bundesimmobiliengesellschaft BIG. Weiters waren die folgenden Partnerorganisationen beteiligt:

* AEE - Institut für nachhaltige Technologien

* BAI Bauträger Austria Immobilien GmbH

* Bundesimmobiliengesellschaft

* Bundesinnung Bau

* ENERTEC Naftz \& Partner OG

* Fronius International $\mathrm{GmbH}$

* MA 39: Prüf-, Überwachungs- und Zertifizierungsstelle der Stadt Wien

* TU Wien, Institut für Thermodynamik und Energiewandler 
TU Wien, Zentrum für Bauphysik und Bauakustik

* Umweltbundesamt $\mathrm{GmbH}$

* TU Wien, Rektorat

In der Vorbereitungs- und Planungsphase wurde auf bestehende Technologieanalysen aufgebaut und ein integriertes Konzept für ein energetisch höchst optimiertes Bürogebäude erstellt. Hierbei wurden alle relevanten Baukomponenten berücksichtigt (Gebäudetechnik, Fassade, Tiefenspeicher etc.).

Ein besonderes Alleinstellungsmerkmal des Projektes lag in der Betrachtung der energieoptimierten Nutzung des Gebäudes. In umfangreicher Detailarbeit wurden ca. 10.000 energieverbrauchende Komponenten im Gebäude erfasst (Heizung, Kühlung, Lift, PCs, Kaffeemaschine, Kopierer, Bewegungsmelder, Sensoren, Steuerungsgeräte uvam.). All diese Komponenten wurden auf ihre Einsparungspotenziale hin untersucht, wobei die gesamten Lebenszykluskosten analysiert wurden. Die Zeitdauer des „Free-Floating-Gebäudebetriebs“ (kein Energiebedarf für Heizen, Lüften und Kühlen) wurde maximiert, zudem ein internes Energiemanagement mit Speichermöglichkeit für den Bedarfs- und Nachfrageausgleich eingerichtet.

Demonstriert wurde das „Büro von morgen“ anhand einer Sanierung des Standortes der TU Wien am Getreidemarkt in der Wiener Innenstadt. Das Gebäude, welches im Herbst 2014 eröffnet wurde, hat 11 Stockwerke, eine Nettogrundfläche von $13.500 \mathrm{~m}^{2}$ und bietet Platz für rund 700 arbeitende Personen. Es handelt sich dabei um das erste Plus-Energie-Bürogebäude Österreichs, das unter Berücksichtigung der Bau- und Betriebsphase rund $90 \%$ weniger Energie als herkömmliche Bürogebäude verbraucht. Zur nachhaltigen Energieversorgung wurde auf dem Dach die derzeit größte Photovoltaik-Anlage Österreichs installiert. Die gesamthafte Energieoptimierung sowie das „Drehen vieler kleiner Schrauben“ machte es möglich, dass die Mehrkosten gegenüber klassischen Bau- und Betriebsformen relativ gering ausfallen.

Aufgrund des innovativen Ansatzes und der hohen Einsparungen wurde dieses Projekt mit zahlreichen Preisen ausgezeichnet, darunter der Staatspreis für Umwelt- und Energietechnologien in der Kategorie "Forschung und Innovation“, die Auszeichnung „Austrian Green Building Star" oder die Aufnahme zu den von 50 „innovativsten Gebäuden Österreichs“ im Jahr 2015.

Der Pioniercharakter des Projektes führte zu einem starken internationalen Interesse. Neben zahlreichen Vorträgen auf Fachkongressen auf der ganzen Welt, die vorrangig durch den wissenschaftlichen Partner wahrgenommen werden, wurden bereits in den ersten Monaten namhafte internationale Delegationen durch das Gebäude geführt, darunter ein Minister aus Hong Kong. 
Eine Gruppe aus China, die Interesse an innovativen Lösungen für Nachhaltiges Bauen hatte, wurde durch das Haus der Zukunft-Programm rasch auf Österreich aufmerksam. Die Auszeichnung des Leitprojektes mit dem Staatspreis führte zu einer Kontaktaufnahme mit dem Leitprojekt-Management. Nunmehr fließt das im Rahmen des Forschungsprojektes generierte Know-how in derzeit zwei Bauprojekte in China ein. Dies unterstreicht, wie öffentliche Investitionen in wirtschaftsnahe Forschung und Entwicklung mittel- und langfristig dazu dienen, die internationale Marktposition österreichischer Unternehmen zu stärken und zusätzliche Wertschöpfung in Österreich zu generieren.

Derzeit wird ein umfangreiches Monitoring des Gebäudes durchgeführt, um daraus weitere Erkenntnisse über die Einsparungen im Alltagsbetrieb zu gewinnen. Angesichts des langen und heißen Sommers im Jahr 2015 sind die Ergebnisse besonders gut - ebenso das Feedback der Nutzer. Der Ansatz, ein Gebäude in all seinen Facetten energetisch zu optimieren, hat sich als sinnvoll erwiesen. Um die damit verbundenen Potenziale vollends erschließen zu können, bedarf es allerdings noch weiterer Forschungarbeiten und einer stärkeren Einbindung der Industrie, bspw. in der Entwicklung stromsparender Komponenten im Gebäude.

\subsubsection{Energy City Graz-Reininghaus}

Ähnlich den Leitprojekten in Salzburg-Lehen und Wien-Aspern richtete „Energy City Graz-Reininghaus" den Fokus auf einen ganzen Stadtteil. Auch hier ging der Ursprung von einem EU-Projekt aus - das Institut für Städtebau an der TU Graz und die Grazer Stadtbaudirektion waren Partner im Central Europe-Projekt "Act4PPP", welches sich dem transnationalen Austausch über Public-PrivatePartnerships zur Bereitstellung öffentlicher Dienstleistungen und Infrastrukturen widmete. Mit der Ausschreibung von Haus der Zukunft-Leitprojekten war die Chance gegeben, das Areal strategisch und innovativ zu entwickeln und dabei die Nachhaltigkeit als Maxime zu setzen. In Verbindung mit der Wirtschaftskrise waren die Grundeigentümer zudem für innovative Ansätze offen, sodass ein steirisches Konsortium eingerichtet werden konnte, an dem neben der Wissenschaft und Verwaltung auch Unternehmen und der Holzcluster Steiermark beteiligt waren.

Das Leitprojektmanagement übernahm das Institut für Städtebau an der Technischen Universität Graz, weiters waren die Stadtbaudirektion, Fachabteilungen des Landes und weitere Institute der Technischen Universität involviert. Für das Demonstrationsvorhaben „Plus Energieverbund Reininghaus Süd“ wurden weitere Partner eingebunden.

Die Entwicklung und Umsetzung von urbanen Strategien für die Neukonzeption, den Bau und Betrieb eines energieautarken Stadtteils war die ursprüngliche In- 
tention des Leitprojektes, das sich an den Ausschreibungszielen orientierte. Es stellte sich im weiteren Verlauf des Projektes jedoch heraus, dass die Energieautarkie eines Stadtteils wenig zielführend ist, da ein Stadtteil nicht losgelöst von der gesamten Stadt und ihrem Umland betrachtet werden kann. Auf diese Erkenntnis wurde in weiteren Ausschreibungen Bezug genommen.

Davon unbehelligt blieb das Bestreben, ein nachhaltiges Gesamtenergiekonzept für den Stadtteil Graz-Reininghaus zu entwickeln. Im Rahmenplan „Energy City Graz-Reininghaus" wurde der Entwicklungsprozess für die energieoptimierte Neugestaltung des Quartiers initiiert und begleitet, bei dem energetische Zielwerte entwickelt und in lokale Pläne integriert wurden. Ferner beinhaltet der Rahmenplan Handlungsempfehlungen für zukünftige energieoptimierte Stadtteilentwicklungen in Graz und der Steiermark und damit eine wertvolle Wissensbasis für zukünftige energieoptimierte Stadt(teil)entwicklungen. Inhaltlich spricht der Rahmenplan folgende Aspekte an:

* Energieverbrauch und Energiebereitstellung (für Kerngebiet, Wohnbau und Gewerbe)

* Energieverteilung (Ansatz:Vernetzung von Gebäuden)

* Städtebau (Aktivierung energetischer Potenziale, Geothermie, Bebauungsdichte, optimale Baukörperstellung, solare Aktivierung von Dächern und Fassaden, Prozesswärmenutzung, Blockheizkraftwerke, Einspeisung überschüssiger Energie in kommunale Netze)

* Gebäude (Anbindung an Kühlenergiepotenzial vorhandener Kühlkeller, energieoptimierte Fassaden, Speichermassen im Inneren, Reduzierung Kühlenergiebedarf)

* Technische Gebäudeausrüstung (energiesparende Heizungs- und Lüftungssysteme, Vermeidung von Klimaanlagen)

Zur Untermauerung des Konzeptes wurde ein Demonstrationsvorhaben als internationales Leuchtturmprojekt realisiert. Mit dem „Plus Energieverbund Reininghaus Süd" sollte dargestellt werden, dass eine wirtschaftlich umsetzbare, technisch und organisatorisch innovative Lösung für Plusenergieverbundkonzepte möglich ist. Der Plusenergieansatz findet dabei nicht auf Ebene des einzelnen Gebäudes sondern innerhalb eines multifunktionalen Gebäudeverbundes statt. In einem ersten Schritt wird das einzelne Gebäude optimiert und wandelt sich vom Energieverbraucher zum Energieerzeuger, im zweiten Schritt bringen Synergien innerhalb des Gebäudeverbundes eine weitere Optimierung des Systems.

Das nach dem Gesamtenergiekonzept geplante Quartier hat eine Bruttogeschossfläche von insgesamt $22.918 \mathrm{~m}^{2}$. In dem Quartier sind 177 Wohneinheiten, davon 34 als „Betreutes Wohnen“, ein Supermarkt sowie Gastronomie- und Büroflächen 
untergebracht. Erstmals wurde ein fünfgeschossiger Holzbau in Österreich durchgeführt. Angedacht war eine Errichtung der Gebäude in vier Bauabschnitten, drei davon sollten auf den Wohnbau entfallen. Aufgrund des großen Erfolges und der enormen Nachfrage nach den Wohnungen wurden der Bauabschnitt 2 und 3 zusammengelegt, sodass die Wohnungen früher bezogen werden konnten.

Die Wirkung des Leitprojektes „Energy City Graz-Reininghaus“ unterstreicht diese hohe Nachfrage von Bewohnern. Das Projekt verdeutlichte den Mehrwert Nachhaltigen Bauens nicht nur aus ökologischen und ökonomischen Motiven, sondern die dadurch mögliche Steigerung des Wohnkomforts. Die Entwicklung des gesamten Stadtteils anhand nachhaltiger Kriterien steigerte das Bewusstsein auch bei der öffentlichen Hand und Unternehmen wie bspw. Bauträgern. Hierbei kommt dem Projekt zugute, dass ein wirtschaftlich besonders gut abbildbarer Low-techAnsatz verfolgt wurde, der stets auch die Kostendimension berücksicht hat.

Des Weiteren haben Interessenten aus dem Ausland das Projekt mehrfach besichtigt. Zu erwähnen ist dabei eine japanische Delegation, die drei Projekte in Europa in Europa besichtigte: London, Stockholm und Graz-Reininghaus. Vorträge und Präsentationen auf internationalen Ausstellungen unterstreichen auch, dass das Projekt durchaus vergleichbar ist mit jenen in anderen Ländern. In wirtschaftlicher Hinsicht war die Zusammenarbeit mit dem Holzcluster von großer Bedeutung, da der Cluster das Projekt auf Fachmessen u.dgl. im Detail vorstellen und seinen Mitgliedsunternehmen damit Marktchancen im In- und Ausland eröffnen konnte.

Den Fokus der Aufmerksamkeit vom Einzelgebäude auf ein ganzes Stadtviertel anzuheben hat sich als sehr zukunftsweisend herausgestellt. Mit dem Programm "Stadt der Zukunft" wurde auch in der Forschungs- und Innovationsförderung dieser Schritt vollzogen. Eine "Stadt der kurzen Wege" hat in ökologischer Hinsicht viele Vorteile, zB weil die Energie nicht nur in Wohn-, sondern auch in Geschäftsund Bürogebäuden genutzt wird und sich somit der Energieverbrauch gleichmäßiger über den Tag verteilt. Nichtsdestotrotz gibt es noch viele rechtliche, wirtschaftliche und technische Fragestellungen in diesem Zusammenhang.

Abschließend ist zu erwähnen, dass die Entwicklung eines auf Nachhaltigkeit hin optimierten Stadtteils eine nicht unbeträchtliche Außenwerbung für die Stadt darstellt. Ein Projekt wie jenes in Graz-Reininghaus zu ermöglichen und aktiv zu unterstützen untermauert das Profil einer zukunftsorientierten Stadt. Graz selbst hat in weiterer Folge das Projekt "Smart City Graz" ins Leben gerufen, welches maßgeblich auf das Haus der Zukunft-Leitprojekt in Reininghaus zurückgeht. 


\subsubsection{Gesamthafte Betrachtung der Leitprojekte}

In gesamthafter Betrachtung der acht Leitprojekte lassen sich einige Aspekte und Erkenntnisse festhalten, die nicht nur in Bezug auf Haus der Zukunft, sondern auch für andere Forschungsprogramme von Relevanz sind.

Das Instrument der Leitprojekte war ein besonders innovativer Ansatz in der Forschungsförderung. Der Zeitpunkt für die Ausschreibung am Beginn der zweiten Programmphase war richtig gewählt, denn es hatte sich bereits eine Fachcommunity in Österreich gebildet, welche für Projekte in dieser Dimension erforderlich ist. Für die Umsetzung von Leitprojekten waren Partner aus Wirtschaft und Forschung verpflichtend, sodass hiermit ein weiterer Stimulus für die Kooperation gesetzt wurde. Auch wurden in zahlreichen Forschungsprojekten jenes Know-how aufgebaut und jene Technologien entwickelt, die in den Leitprojekten angewendet wurden. Hätte man die Ausschreibung einige Jahre früher durchgeführt, so wären mit Sicherheit nicht derart innovative und erfolgreiche Projekte zustande gekommen.

Die Umsetzung von Leitprojekten dieser Größenordnung war für alle Beteiligten Neuland und brachte dementsprechende organisatorische und administrative Fragestellungen mit sich. So wurden etwa lediglich die Mehrkosten für die innovativen Aspekte des Bauvorhabens gefördert. Einigen Leitprojektmanagern war nicht erkennbar, wie genau dieser Innovationsgehalt definiert war. Im Spannungsfeld zwischen einer möglichst breit angelegten Ausschreibung, um alle Akteure und Projekte ins Boot zu holen, und der Detailentscheidung im Zuge der Projektgenehmigung war es erforderlich, gewisse Einzelfallentscheidungen zu treffen.

Leitprojekte eröffneten die Möglichkeit, komplexe Themenstellungen in ihrer Breite zu behandeln. Auch wurde der Schritt von der Forschung in die Praxis unmittelbar vollzogen. Damit wurde nicht nur der faktischen Komplexität der Bauthematik mit all ihren Facetten Rechnung getragen, sondern auch die Forschung vor die Herausforderung gestellt, neue Lösungen zu entwickeln, welche sich unmittelbar anwenden lassen. Die Chancen und Probleme im Zusammenhang mit innovativen Gebäudelösungen, die sich in der praktischen Umsetzung gezeigt haben, wurden somit rascher sichtbar als in klassischen Disseminationsprozessen.

Im Rahmen der Leitprojekte wurde Forschung und Innovation im Bereich Nachhaltigen Bauens sichtbar und begreifbar gemacht. Forschung und Innovation wurde damit vom ideellen „theoretischen“ Ergebnis zum realen Gebäude. Damit werden nicht nur die praktischen Vor- und Nachteile neuer Bauansätze sichtbar gemacht, sondern es werden auch die Kompetenzen der beteiligten Akteure demonstriert. Dies dient auch der wirtschaftlichen und wissenschaftlichen Positionierung Österreichs als Kompetenzträger in diesem Sektor. Darüber hinaus macht es Entscheidungsträgern in Politik und Verwaltung sowie der breiten Öffentlichkeit die Machbarkeit und Vorteile nachhaltiger Bauformen bewusst. 
Nachhaltiges Bauen wurde in seiner Gesamtheit betrachtet, zu der neben technologischen Fragestellungen auch jene der Kosten und Finanzierung, der Nutzerakzeptanz, der rechtlichen Rahmenbedingungen uäm. zählen. Mit der Entwicklung einer Technologie ist noch nichts für Klima- und Umweltschutz getan - erst mit der Anwendung werden Effekte erzielt. Um dies zu erreichen braucht es, auch im Zuge eines Forschungsprogrammes, eine umfassende Beschäftigung mit allen Hemmnissen und Problemen in der Praxis. Fragen der Finanzierbarkeit sind dabei von besonders hoher Relevanz. Hierbei wurde bspw. das Problem offensichtlich, dass die Mehrinvestitionen für nachhaltige Technologien in aller Regel der Bauherr trägt, den damit verbundenen Vorteil niedrigerer Betriebskosten trägt jedoch der Mieter. Damit unterscheiden sich Innovationen im Bau essentiell von industriellen Innovationen, wo der Produzent auf dieser Grundlage neue Produkte entwickeln und damit Wettbewerbsvorteile erschließen kann. Auch die Finanzierung betrieblicher Investitionen war eine Herausforderung, etwa, dass die Mehrkosten für nachhaltige Baulösungen von Banken nur in geringem Ausmaß berücksichtigt werden.

Ein sehr großer Mehrwert wurde durch die Leitprojekte in Bezug auf die internationale Sichtbarkeit Österreichs erzielt. Die Leitprojekte machen deutlich, welche Kompetenzen österreichische Akteure in diesem Themenfeld haben und wie eine konkrete Realisierung aussehen kann. Fast alle Leitprojekte wurden mehrfach von internationalen Delegationen aus aller Welt besucht. Dadurch wurden österreichischen Anbietern neue Märkte erschlossen, bspw. in China. Für Wissenschafter waren die Haus der Zukunft-Leitprojekte vielfach die "Eintrittskarte" in internationale Forschungsverbünde, etwa im EU-Programm Horizon 2020. Viele nationale und internationale Auszeichnungen unterstreichen die Qualität und den Leuchtturmcharakter der Projekte.

So wurden aus den Leitprojekten viele wichtige Erkenntnisse gewonnen - zugleich traten neue Fragen auf, bei denen ein weiterführender Forschungsbedarf gegeben ist. Dies betrifft neben Details zu technischen Lösungen insbesondere die Nutzerintegration, die Finanzierbarkeit nachhaltiger Konzepte, die Vereinbarkeit mit rechtlichen Rahmenbedingungen und mit den Anforderungen des sozialen Wohnbaus, die Vernetzung mit anderen Gebäuden, Stadtteilen oder Energiesystemen etc.

Dem Leuchtturmcharakter der Leitprojekte entsprechend haben sich dabei jene Akteure zusammengefunden, die über besondere Kompetenzen im Bereich des Nachhaltigen Bauens verfügen. Auch wurden die Projekte ausgewählt und realisiert, die unter relativ günstigen Voraussetzungen entwickelt wurden. Die zentrale Herausforderung für die Zukunft ist nun, diese Exzellenz auf die gesamte Breite des österreichischen Bausektors zu übertragen, damit die erprobten Lösungen und Technologien zumindest teilweise auch in der breiten Masse an Bauvorhaben Anwendung finden. 


\subsection{Begleitmaßnahmen}

Neben der Umsetzung von Leitprojekten liegt ein weiteres Alleinstellungsmerkmal von Haus der Zukunft im Vergleich zu anderen Forschungsprogrammen in der starken Betonung von Begleitmaßnahmen. Diese Maßnahmen stehen in unmittelbarem Zusammenhang mit den Programmzielen, die nicht nur die Technologieentwicklung, sondern auch den Aufbau einer entsprechenden Community, die Forcierung der Anwendung und Marktdurchdringung innovativer Lösungen, den Kompetenzaufbau uäm. adressierten. Nachfolgend werden die dabei umgesetzten Maßnahmen und erzielten Wirkungen zusammengefasst.

\section{* Informationsveranstaltungen zum Förderprogramm}

Im Vorfeld zu den Ausschreibungen wurden in ganz Österreich Informationsveranstaltungen abgehalten, bei denen das Programm bzw. die jeweilige Ausschreibung vorgestellt wurden. Dies war zu Beginn von Haus der Zukunft ein innovativer Ansatz - mittlerweile führen nationale Förderagenturen einen kontinuierlichen Dialog mit Einrichtungen auf Länderebene und setzen gemeinsame Maßnahmen.

Die Auswertung der Projektanträge zeigt, dass eine Vielzahl von Einrichtungen unterschiedlicher Art, zum Großteil Unternehmen, an dem Programm teilgenommen haben. Auch konnten Akteure aus allen Bundesländern für die Programmteilnahme gewonnen werden, wenngleich das Gros an Projekten in vier Bundesländern angesiedelt ist (Wien, Steiermark, Nieder- und Oberösterreich).

\section{* Beratungsangebote / Beratungsgespräche}

Nicht zuletzt aufgrund dieser Informationsangebote konnte ein hohes Interesse an den Ausschreibungen für Haus der Zukunft generiert werden. Um interessierte Akteure optimal beraten zu können und den zeitlichen Aufwand für die Antragstellung so gering wie nötig zu halten, wurden Beratungsgespräche angeboten bzw. Beratungen per Telefon und Email ermöglicht.

Im Vorfeld der Ausschreibungen wurden zum Teil hunderte solcher Beratungen durchgeführt. Das Angebot von Pre-Proposal-Checks durch die ÖGUT, welche die meisten Begleitmaßnahmen operativ umgesetzt hat, wurde von bis zu 120 Akteuren in Anspruch genommen.

\section{Vernetzungsworkshops im Rahmen der jeweiligen Ausschreibungen}

Mit den Projektträgern neuer und laufender Projekte in einem ähnlichen Themenbereich wurden im Rahmen der jeweiligen Ausschreibungen Vernetzungs- 
workshops durchgeführt, um Kooperations- und Synergiepotenziale zwischen den Projekten zu erschließen. Dabei wurden die Projekt bzw. deren bisherige Ergebnisse von den Projektträgern vorgestellt und ein Rahmen für den zielgerichteten Austausch geschaffen.

Mit den Leitprojektmanagern wurden insbesondere zu Beginn der Leitprojekte separate Workshops abgehalten. Damit konnten viele administrative Fragen auf kurzem Weg geklärt werden. Ferner wurde damit die Basis für eine intensive Zusammenarbeit zwischen Leitprojektpartnern, auch über das geförderte Projekt hinaus, initiiert.

\section{Themenspezifische Workshops (zB Haus der Zukunft in der Praxis)}

Ergänzend wurden themenspezifische Workshops unter dem Titel „Haus der Zukunft in der Praxis" abgehalten, bei denen erfolgreiche Projekte öffentlich präsentiert wurden. Vier Projekte mit einer ähnlichen Themenstellung wurden dabei gebündelt und jeweils in einem ca. halbstündigen Vortrag vorgestellt.

Diese Formate hatten zum Ziel, die Projektergebnisse einem interessierten Fachpublikum zugänglich zu machen und aktiv zum Community Building beizutragen. Durch die thematische Fokussierung wurde ein für die jeweiligen Fachleute attraktiver Überblick über die aktuelle Forschung in Österreich in einem speziellen Themenfeld geboten und der Transfer der Ergebnisse in die Praxis unterstützt sowie die Diskussion mit anderen Akteuren unterstützt.

\section{* Online-Plattform www.hausderzukunft.at}

Bereits 1999 wurde durch das BMVIT eine Open Access Online-Plattform ins Leben gerufen und seitdem kontinuierlich weiterentwickelt. Über die Übersichtsseite www.nachhaltigwirtschaften.at ist ein rascher Einstieg in die verschiedenen Programme und Themenfelder der Initiative, so auch Haus der Zukunft, möglich. Diese Plattform verzeichnet rund 1.500 Projektbeschreibungen und 1.320 Publikationen und weist pro Monat zwischen 15.000 und 30.000 Zugriffe auf.

Die Website www.hausderzukunft.at diente neben der Verbreitung von fachlichen Erkenntnissen auch als Plattform für die Community. Aktuelle Highlights, Veranstaltungen und Termine wurden auf diesem Wege breit gestreut. Die Mailadresse office@hausderzukunft.at wurde für Erstauskünfte genutzt und laufend redaktionell betreut.

Bereits bei Projektbeginn wurden eine Projektskizze, ein englischsprachiger Abstract und die Kontaktinformationen des Projektträgers auf der Homepage publiziert, um Synergien zu anderen Projekten zu erschließen. Ein besonders innovativer Aspekt war die Veröffentlichung aller Projektberichte nach Projekt- 
abschluss. Dies ist heute in vielen Programmen gang und gäbe - zum damaligen Zeitpunkt war Haus der Zukunft eines der ersten Programme in Österreich und Europa, das Publizität konsequent umgesetzt hat.

Die konsequente Publizität der Projektergebnisse hat das gewonnene Wissen breit zugänglich gemacht. Auch konnte dadurch eine hohe Qualität der Berichte sichergestellt werden.

Neben der Programmwebsite wurden auch weitere Maßnahmen zum Wissenstransfer über neue Medien gesetzt. Erwähnenswert ist hier die Open ContentPlatformm „e-genius.at", die im Rahmen von Haus der Zukunft umgesetzt wurde. „e-genius.at" ist eine Lehr- und Lernplattform mit frei zugänglichen Bildungsmaterialien für für Schulen, Fachhochschulen, Universitäten, Erwachsenenbildung sowie für Privatpersonen, die sich selbstorganisiert weiterbilden möchten. Sie bietet anschauliche, praxisbezogene Fachtexte zu den Themen Energieeffizientes Gebäude und Erneuerbare Energien. Aktuell stehen 27 Lernfelder unterteilt in 200 Kapitel bzw. Lernbausteine zur Verfügung. Dies entspricht einem Umfang von mehr als 1.000 didaktisch gestalteten Textseiten.

Die Plattform wurde sowohl national wie auch international mehrfach ausgezeichnet (zB "Energy Globe Award Vienna" in der Kategorie Jugend, COMENIUS-Award für digitale Bildungsmedien).

\section{* Öffentlichkeitsarbeit}

Haus der Zukunft hatte auch zum Ziel, die Ergebnisse der Forschungsprojekte einer breiten Öffentlichkeit zu kommunizieren, um dadurch den Wissenstransfer zu unterstützen und ein größeres Bewusstsein für die Relevanz und Potenziale des Themenfeldes „Nachhaltig Bauen“ zu schaffen. Daher wurden von Professionisten entsprechende Kommunikationsmaßnahmen konzipiert und umgesetzt. Dazu wurde das klassische Spektrum an Kommunikationsinstrumenten wie Newsletter, Presseaussendungen, Veranstaltungen u.dgl. eingesetzt.

Zur Verbreitung der Forschungsergebnisse wurden Kurzbeschreibungen aller Projekte auf Deutsch und Englisch, Veranstaltungsdokumentationen uäm. auf der Website verfügbar gemacht. Endberichte von abgeschlossenen Projekten wurden in der Schriftenreihe "Berichte aus Energie- und Umweltforschung“ publiziert.

Für die Demonstrations- und Leitprojekte wurden spezifische PR-Maßnahmen gesetzt, etwa durch Ausrichtung einer medienwirksamen Feier zur Grundsteinlegung und/oder Eröffnung des Gebäudes mit Verleihung einer Haus der Zukunft-Plakette. Damit wurde das Programm Haus der Zukunft und das Themenfeld Nachhaltiges Bauen auch gezielt in den Regionen Österreichs sichtbar gemacht. 


\section{Kooperationsveranstaltungen}

Bei zahlreichen Kongressen, Symposien und ähnlichen Veranstaltungen wurden das Programm und Ergebnisse aus geförderten Projekten vorgestellt. Exemplarisch genannt seien die folgenden Veranstaltungen: Symposium AktivSolarhaus, Internationale Passivhaustage, Eurosun, Smart Cities Week Wien, SET-Plan Konferenzen, World Sustainable Energy Days, Eurosun-Konferenz, Sustainable Buildings Conference, Solar Decathlon, BauZ!-Kongress, "Best of Haus der Zukunft-Konferenz" (in Zusammenarbeit mit WKO-Fachverband Steine-Keramik) etc.

Bei manchen Veranstaltungen, die sich gezielt an ein relevantes Fachpublikum richteten, wurde Haus der Zukunft im Rahmen einer eigenen Session beleuchtet. Damit konnten Synergien in Hinblick auf die Ansprache von Zielgruppen erschlossen werden.

Ferner wurde Haus der Zukunft auf zahlreichen Fachveranstaltungen im Inund Ausland vorgestellt. Bei der Präsentation von Ergebnissen aus geförderten Projekten bei wissenschaftlichen Kongressen oder Fachmessen wurde auf das Förderprogramm hingewiesen, sodass das Programm Haus der Zukunft Bekanntheit über die Grenzen Österreichs hinaus erlangte.

Insbesondere die Vorstellung von Haus der Zukunft als europäisches Best Practice-Modell im Europäischen Parlament ist hierbei erwähnenswert. Haus der Zukunft wurde 2009 bei einer Enquete zur EU-Gebäuderichtlinie, welche damals in Erstellung war, auf Initiative des Luxemburger Abgeordneten Claude Turmes vorgestellt und fand große Beachtung.

\section{* Publikationen (zB Forschungsforum, Technical Guide,...)}

Ein besonderer Schwerpunkt wurde auf die Verbreitung der Forschungsergebnisse gelegt, um das neu gewonnene Wissen breit zugänglich zu machen. Dies sollte dazu beitragen, die österreichischen Kompetenzen im Bereich des Nachhaltigen Baues zu vertiefen und Ideen für neue Projekte und Themenschwerpunkte anzustoßen. Daher wurden Publikationen in unterschiedlichen Formaten erstellt und an die relevanten Zielgruppen kommuniziert.

Folgende Publikationsformate kamen dabei zur Anwendung:

$>$ Publikation der Endberichte zu den geförderten Projekten

> Themenbezogene Publikationen wie bspw. "Nachhaltiges Bauen Made in Austria“ (Monitoring der Demonstrationsgebäude), "Neue Energietechnik für Häuser mit Geschichte" (Zeitgemäße Sanierung von Gebäuden unter Denkmal- oder Ortsbildschutz) 
> Broschüren wie "Innovative Gebäude in Österreich - Technical Guide“, "Best of Haus der Zukunft“, „10 Jahre Programmlinie Haus der Zukunft"

$>$ Diverse Beiträge in der Publikationsreihe „Forschungsforum Nachhaltig Wirtschaften“ des BMVIT

$>$ Diverse Beiträge im Magazin „Energy Innovation Austria“ von BMVIT und Klima- und Energiefonds

$>$ DVDs: „Highlights aus der Energieforschung" (Solar Decathlon und "Energie-Experiment" Güssing), „S-HOUSE - Schiestlhaus - Lust auf Lehm“ (Dokumentation zweier einzigartiger Gebäude und Fernsehdokumentation über den Baustoff Lehm), „Bauen mit Hausverstand - Das Haus der Zukunft“ (Dokumentation von aktuellen Pilotprojekten) ${ }^{5}$, "Renovation with Innovation - Die Chancen intelligenter Sanierung“

$>$ Roll-Ups, Poster, Landkarten uäm.

\section{* Preise / Wettbewerbe}

Zu Beginn des Programms wurden Preise und Wettbewerbe für innovative und nachhaltige Gebäude, Konzepte und Technologien ausgeschrieben. Diese hatten neben der Sensibilisierung und Bekanntmachung des Programms auch zum Ziel, nähere Kenntnisse über den Wissens- und Umsetzungsstand in Österreich zu erhalten, um auf dieser Grundlage die weiteren Schritte in der Programmkonzeptionierung (Instrumente, Themenschwerpunkte etc.) vornehmen zu können.

1999/2000 wurde eine "Auszeichnung für beachtenswerte Pionierleistungen im Wohn-, Büro- und sonstigen Nutzbau in Österreich“ mit dem Fokus auf Neubauten ausgeschrieben, bei der 50 Projekte eingereicht wurden.

2001 folgte der Wettbewerb „Altbau mit Zukunft“, der den neuen Themenschwerpunkt der Althaussanierung gezielt andressierte. 38 Projekte wurden eingereicht und drei erste Preise, drei Auszeichnungen und ein Sonderpreis verliehen. Neun weitere Projekte wurden von der Jury als anerkennungswürdig befunden.

Im Rahmen der „Nachhaltig Wirtschaften Gründungsinitiative“ wurden bei Businessplanwettbewerben 2001 und 2003 jeweils ein Projekt aus dem Themenbereich „Nachhaltiges Bauen“ ausgezeichnet.

5 Die Dokumentation „Bauen mit Hausverstand“ wurde auch auf dem Sender 3sat ausgestrahlt und hatte rund 700.000 Zuseher im gesamten deutschen Sprachraum. 


\section{OUTCOME: Wirkung des Programms}

Der vierte Abschnitt des Evaluierungsberichts beleuchtet den OUTCOME. Damit sind die indirekt erzielten Wirkungen des Förderprogramms erfasst, die sich aus den direkten Ergebnissen wie insbesondere den geförderten Projekten ergeben haben.

Um diese Wirkungen zu erfassen wurde eine Onlinebefragung unter allen Programmteilnehmern durchgeführt. Weiters wurden Schlüsselpersonen - Stakeholder, ausgewählte Fördernehmer und internationale Experten - im Rahmen von Interviews befragt. Weiters wurde eine Berechnung der volkswirtschaftlichen Effekte durchgeführt. Eine zusammenfassende Wirkungsanalyse fasst die Kernergebnisse zusammen.

\subsection{Onlinebefragung der Programmteilnehmer}

Alle Projektträger am Programm Haus der Zukunft wurden im Zuge der Evaluierung eingeladen, ihre Einschätzung des Programms und seiner Wirkungen im Rahmen einer Onlinebefragung einzubringen. Dafür wurde zwischen drei Gruppen unterschieden - Unternehmen, Forschungseinrichtungen und sonstige Einrichtungen - und mit individuell, jedoch ähnlich strukturierten Fragebögen befragt. Die Fragebögen sind im Anhang zum Evaluierungsbericht angeführt.

Da manche Akteure mehrfach an Haus der Zukunft-Projekten teilgenommen haben, wurden 452 Personen angeschrieben. Davon waren 31 Emails nicht zustellbar, sodass 421 Personen die Möglichkeit zur Teilnahme hatten. 84 Personen haben sich daraufhin an der Onlinebefragung beteiligt, was eine Rücklaufquote von $20 \%$ ergibt. Dies ist in Anbetracht des Zeitaufwandes ohne Gegenleistung ein relativ hoher Wert und unterstreicht der zum Teil schon lange zurückliegende Projektzeiträume und Interesse der Beteiligten, Feedback zum Programm abzugeben.

In weiterer Folge werden die Ergebnisse der Onlinebefragung in den drei Gruppen zusammenfassend dargestellt. Aus Gründen der Übersichtlichkeit werden nur zentrale Grafiken und Auswertungen angeführt - weiterführende Auswertungen finden sich im Anhang zum Evaluierungsbericht. 


\subsubsection{Unternehmen}

37 Unternehmen haben an der Onlinebefragung teilgenommen, davon sind 23 in der Dienstleistungsbranche tätig (Architektur, Planung). Der Umsatz der befragten Unternehmen hat sich in den letzten Jahren zumeist leicht gesteigert (10 Unternehmen) und bei 6 Betrieben deutlich gesteigert. 6 Unternehmen mussten einen deutlichen und 5 weitere einen leichten Umsatzrückgang verzeichnen. Die meisten Unternehmen sind in Wien ansässig, gefolgt von Niederösterreich und der Steiermark. Bis auf das Burgenland und Kärnten haben Firmen aus allen Bundesländern teilgenommen. Die wichtigsten Absatzmärkte sind Österreich (33 Nennungen) und der EU-Raum (14 Nennungen).

Die befragten Unternehmen haben sich über die gesamte Laufzeit an Haus der Zukunft beteiligt - drei Unternehmen haben bereits bei der ersten Ausschreibung 1999 partizipiert. Mehr als die Hälfte der Unternehmen hat dabei erstmals an einem geförderten Forschungs- und Innovationsprojekt teilgenommen. Es konnten somit unter den Unternehmen auch neue Zielgruppen angesprochen werden.

Abbildung 23: Um welche Art von Projekten handelte es sich dabei? (Mehrfachnennungen möglich)

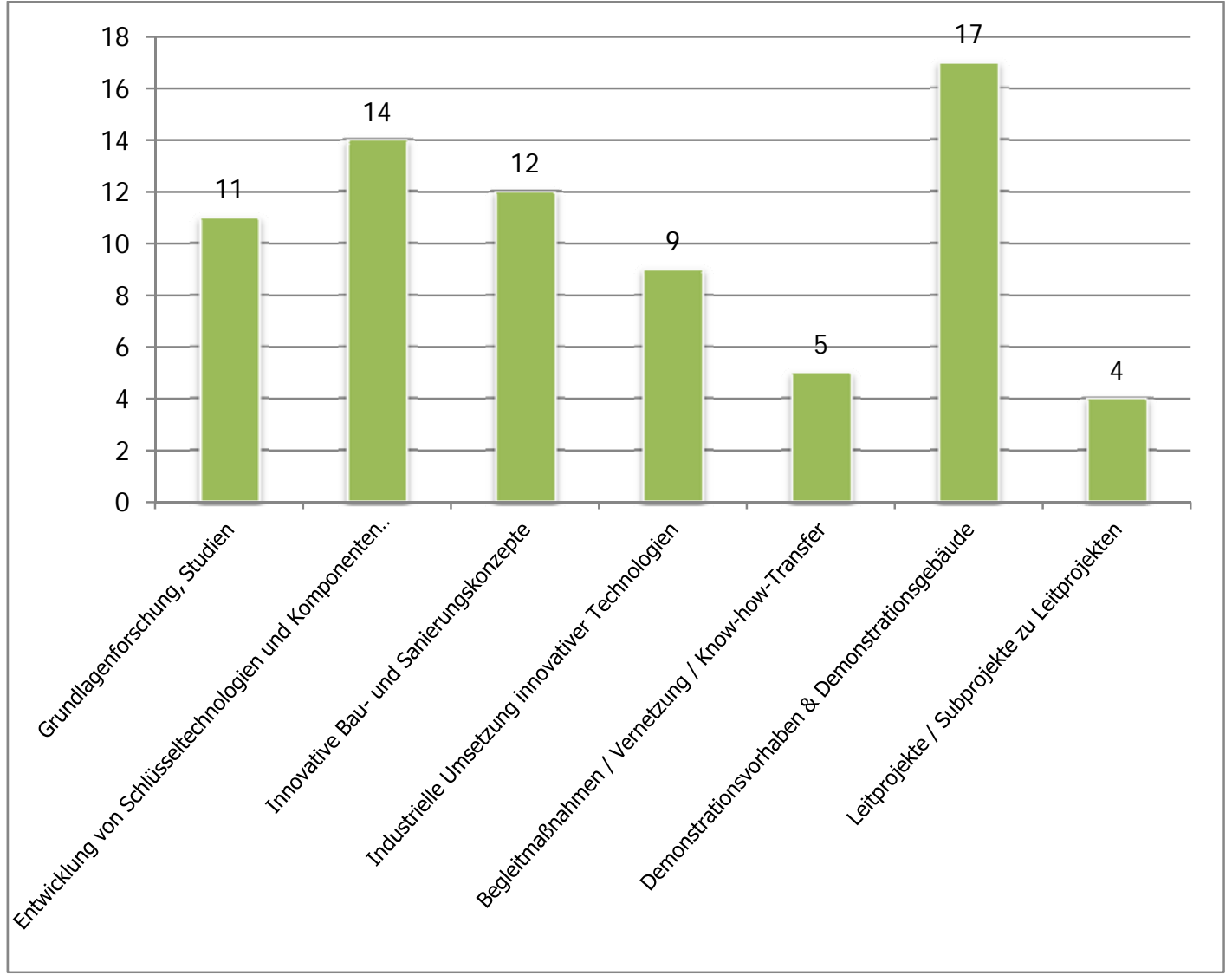

Quelle: Onlinebefragung von 37 Unternehmen; $n=36$ 
Das Spektrum an Projektarten ist ein sehr breites: Die meisten Unternehmen haben sich an Demonstrationsvorhaben und Demonstrationsprojekten beteiligt, gefolgt von der Entwicklung von Schlüsseltechnologien und Komponenten und innovativen Bau- und Sanierungskonzepten. Nur knapp ein Drittel hat sich an der Grundlagenforschung oder der Erstellung von Studien beteiligt.

$\mathrm{Zu}$ den unmittelbaren Auswirkungen des Projektes auf das Unternehmen zählen insbesondere der Aufbau von spezifischem Fachwissen zu einem Thema sowie das Wissen über die Inanspruchnahme von Förderungen und die Zusammenarbeit mit (wissenschaftlichen) Partnern.

Abbildung 24: Welche Auswirkung hatte das Projekt unmittelbar auf das Unternehmen? Bitte geben Sie auf der Skala an, was durch das Projekt in I hrem Unternehmen bewirkt wurde!

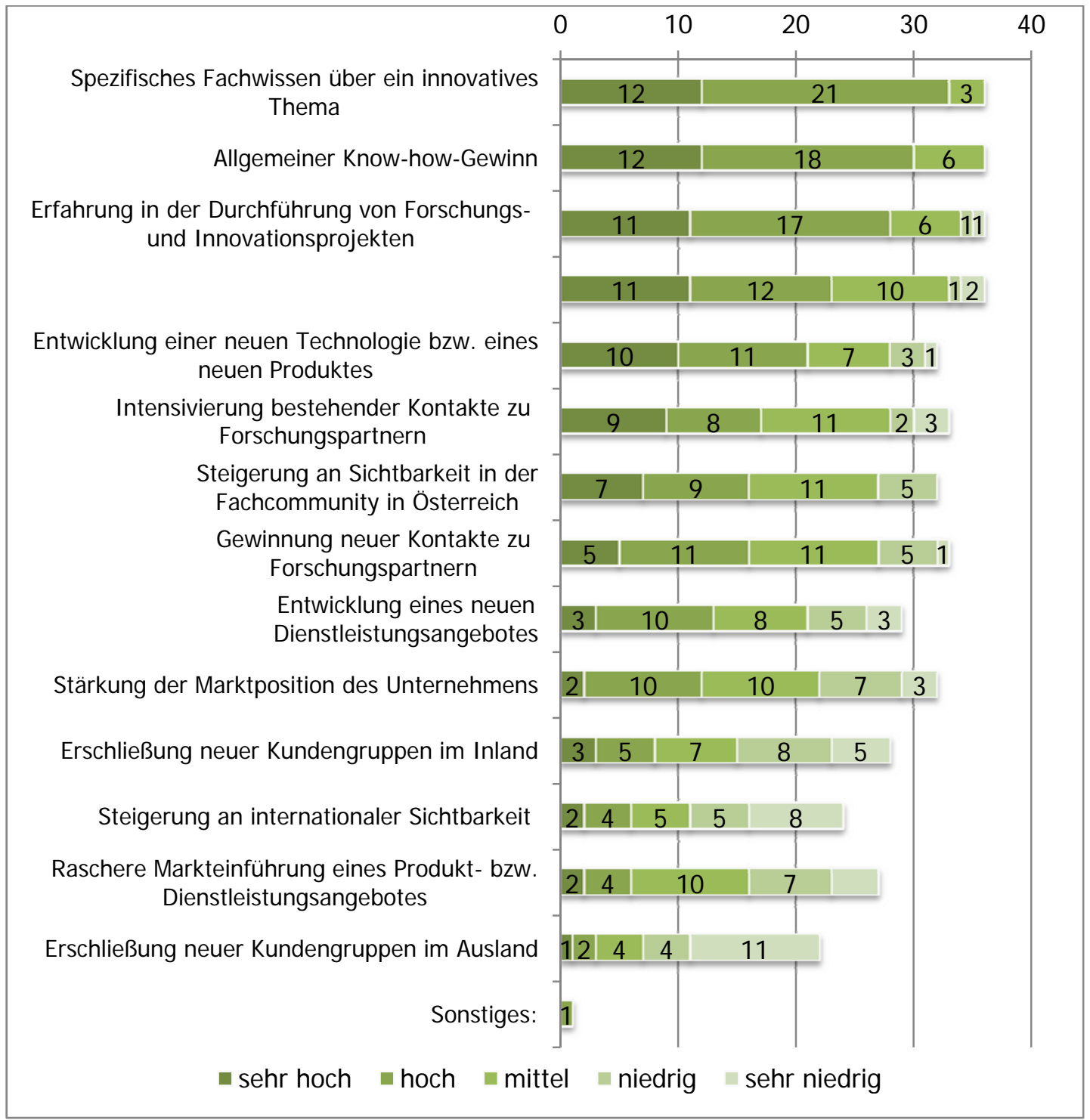

Quelle: Onlinebefragung von 37 Unternehmen; $n=36$ 
In zwei von drei Projekten mit Beteiligung der befragten Unternehmen wurden ein neues Produkt, eine neue Technologie oder ein neues Dienstleistungsangebot entwickelt, was die hohe Praxis- und Wirtschaftsorientierung von Haus der Zukunft unterstreicht. Das Spektrum an Neuentwicklungen ist sehr breit und umfasst Technologien und Komponenten ebenso wie innovative Dienstleistungen (zB Nutzung von Wetterprognosen für die prädikative Gebäudesteuerung).

Abbildung 25: Wurde im Zuge des Projektes ein neues Produkt, eine neue Technologie oder ein neues Dienstleistungsangebot erarbeitet?

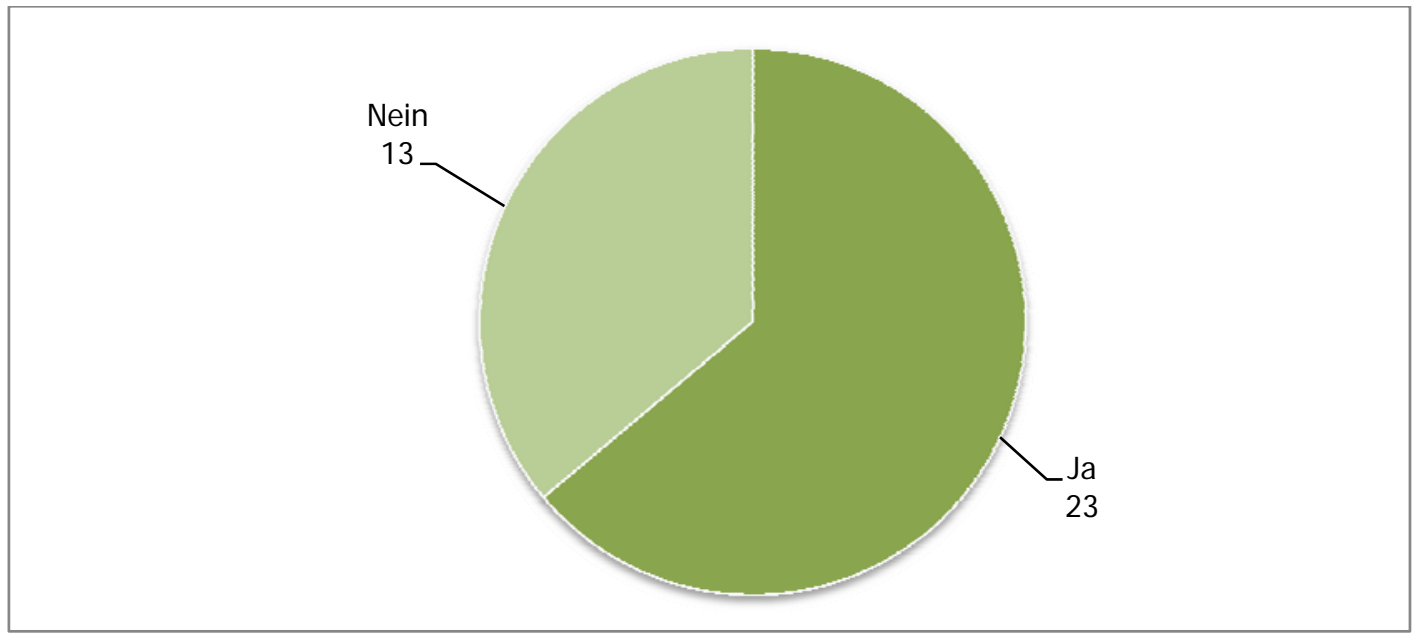

Quelle: Onlinebefragung von 37 Unternehmen; $n=36$

Das neu entwickelte Angebot konnte vorrangig auf regionalen und nationalen Märkten platziert werden. In drei Fällen erfolgte bereits eine internationale Marktüberleitung.

Die Marktakzeptanz war in der Mehrzahl der Fälle erfolgreich oder sehr erfolgreich. Fünf Angebote wurden vom Markt jedoch kaum aufgenommen. Dies ist vor dem Hintergrund zu sehen, das mit innovativen Produkten, Technologien und Dienstleistungen stets ein gewisses Marktrisiko einhergeht.

Abbildung 26: Wie erfolgreich war die Marktakzeptanz dieses neuen Angebotes?

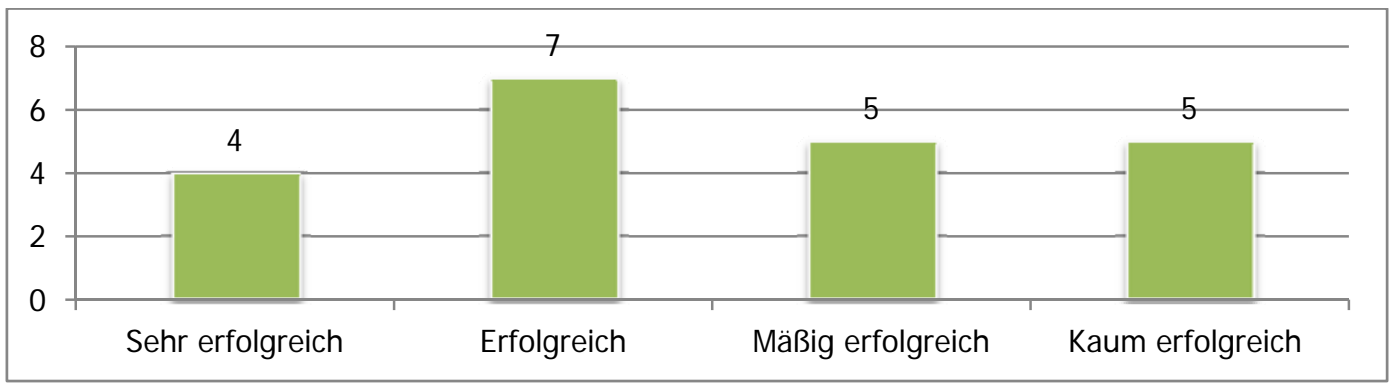

Quelle: Onlinebefragung von 37 Unternehmen; $n=21$ 
Ein Fünftel der befragten Unternehmen konnte dadurch eine signifikante Umsatzsteigerung erzielen. Der zusätzliche Umsatz, der durch die Forschungs- und Innovationsaktivitäten generiert werden konnte, wird auf $€ 200.000$ bis $€ 1,000.000$ geschätzt. Ein Unternehmen gab eine Umsatzsteigerung um $50 \%$ an, also einen beachtlichen Wert.

In einem Drittel der Unternehmen wurden aufgrund der Forschungs- und Innovationsaktivitäten neue Arbeitsplätze geschaffen. Die Anzahl der neu geschaffenen Arbeitsplätze im Unternehmen variiert zwischen 0,25 und 6 - hierbei ist jedoch zu berücksichtigen, dass es sich beim Großteil der befragten Unternehmen um kleinteilig strukturierte, spezialisierte Dienstleistungsunternehmen handelt.

Abbildung 27: Konnten aufgrund der Forschungs- und I nnovationsaktivitäten neue Arbeitsplätze geschaffen werden?

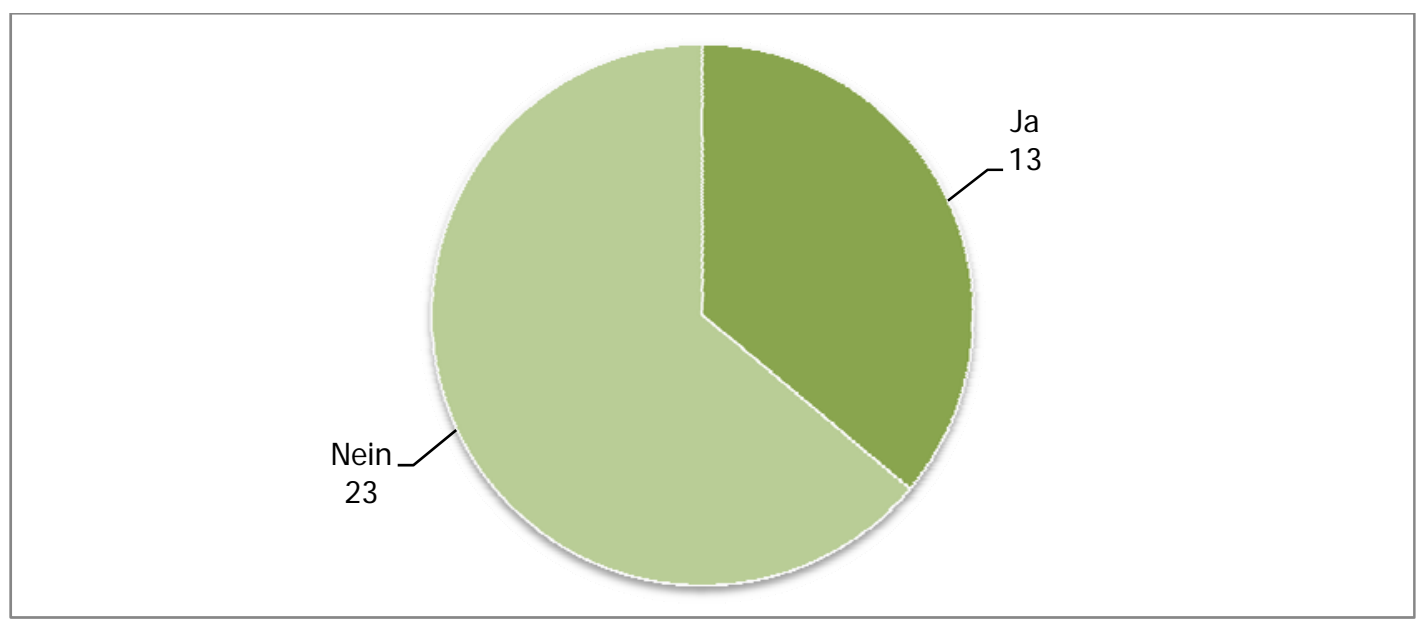

Quelle: Onlinebefragung von 37 Unternehmen; $n=36$

Fast alle Betriebe haben nach Abschluss des Projektes weiter an dem Thema gearbeitet. Die Fortsetzungsaktivitäten können dabei unterschiedliche Formen annehmen: J eweils 13 Unternehmen verwerten die neuen Erkenntnisse im Rahmen der betrieblichen Produktentwicklung bzw. in der Einführung eines neuen Produktund Dienstleistungsangebotes. 11 Unternehmen haben ein Forschungs- und Innovationsprojekt in einem anderen Förderprogramm durchgeführt, 9 haben ohne Förderung weiter in dem Themenfeld geforscht. 5 Unternehmen haben im unmittelbaren Anschluss ein weiteres Haus der Zukunft-Projekt durchgeführt. 
Abbildung 28: Haben Sie nach Abschluss des geförderten Projektes weiter an dem Thema gearbeitet?

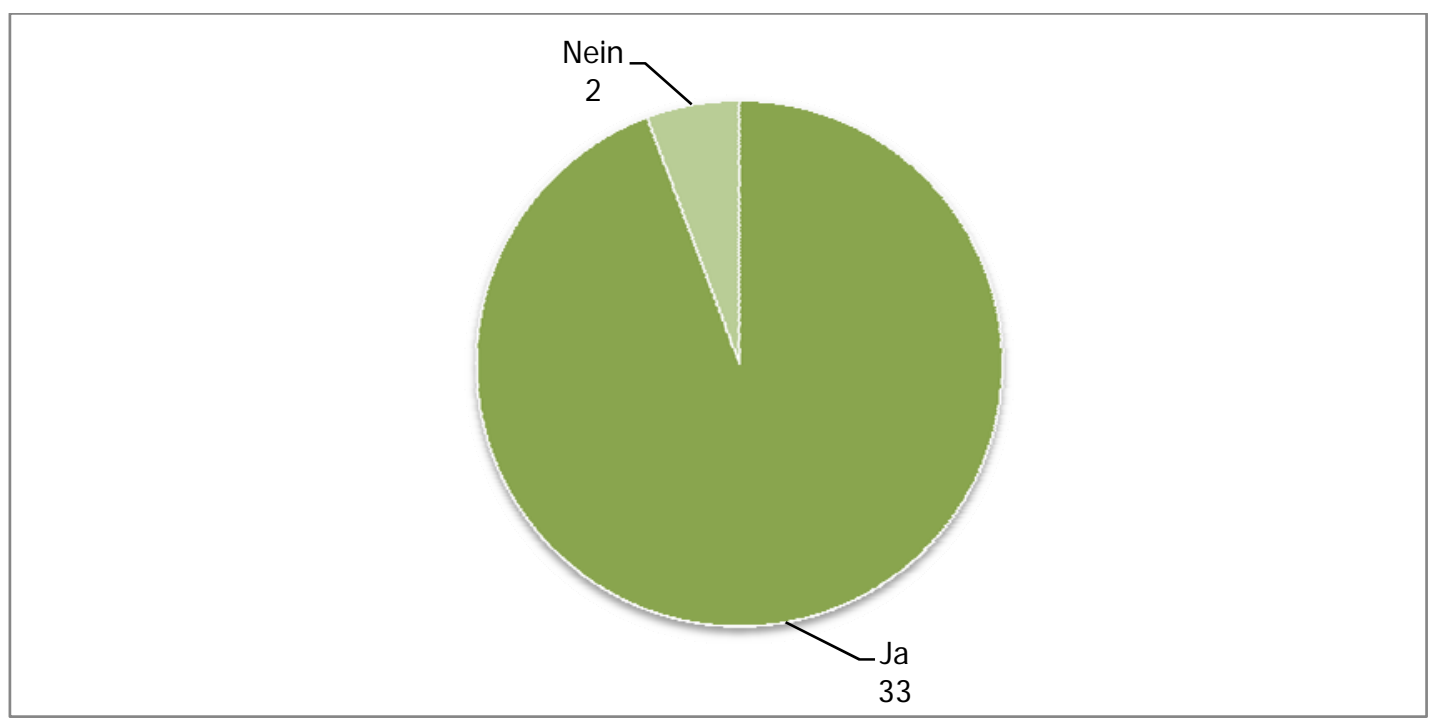

Quelle: Onlinebefragung von 37 Unternehmen; $n=35$

Abbildung 29: In welcher Form? (Mehrfachnennungen möglich)

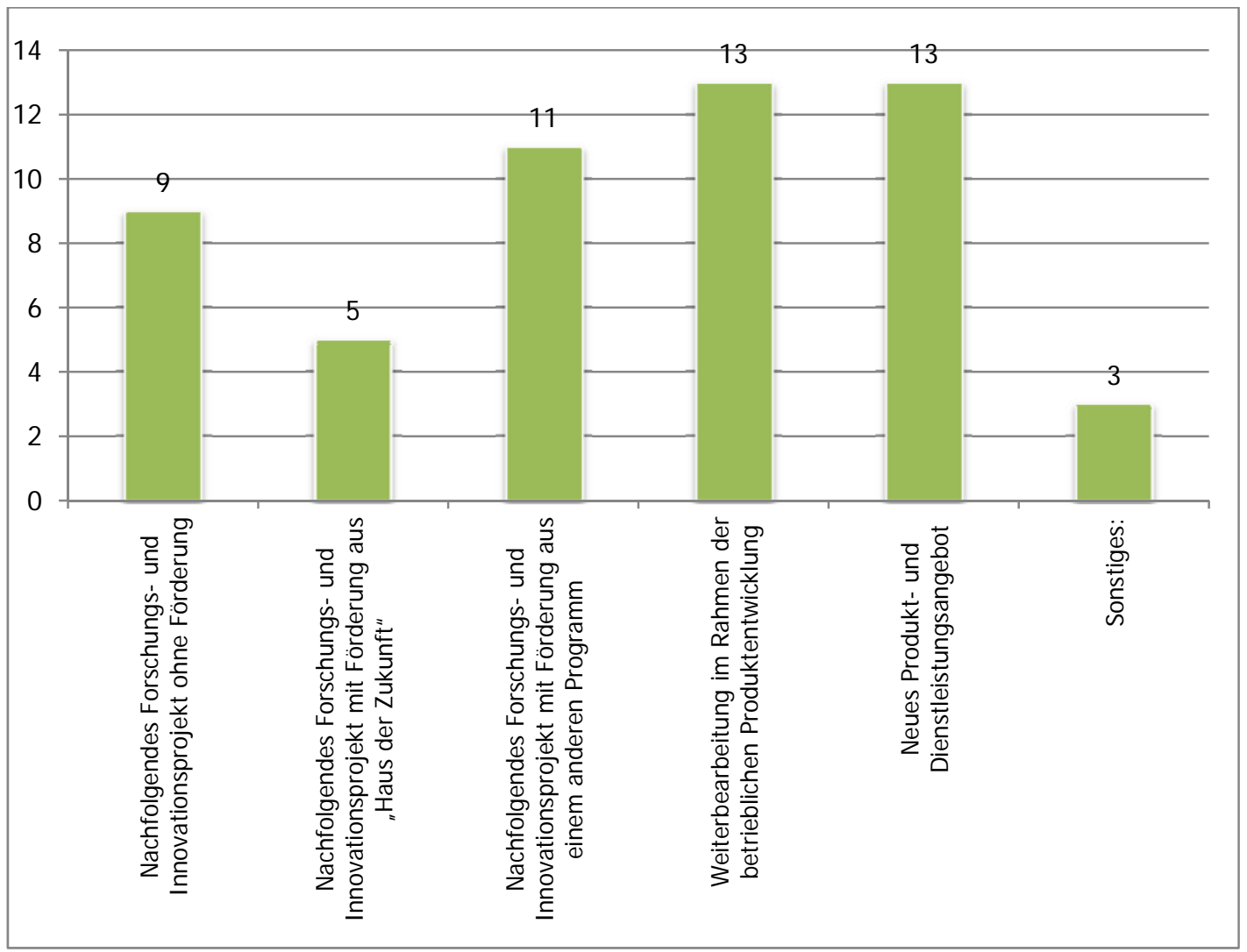

Quelle: Onlinebefragung von 37 Unternehmen; $n=32$ 
Abbilldung 30: Was konnte nach I hrer Wahrnehmung durch das Programm Haus der Zukunft insgesamt in Österreich bewirkt werden? Bitte geben Sie auf der Skala an, wie hoch Sie die Wirkung einschätzen!

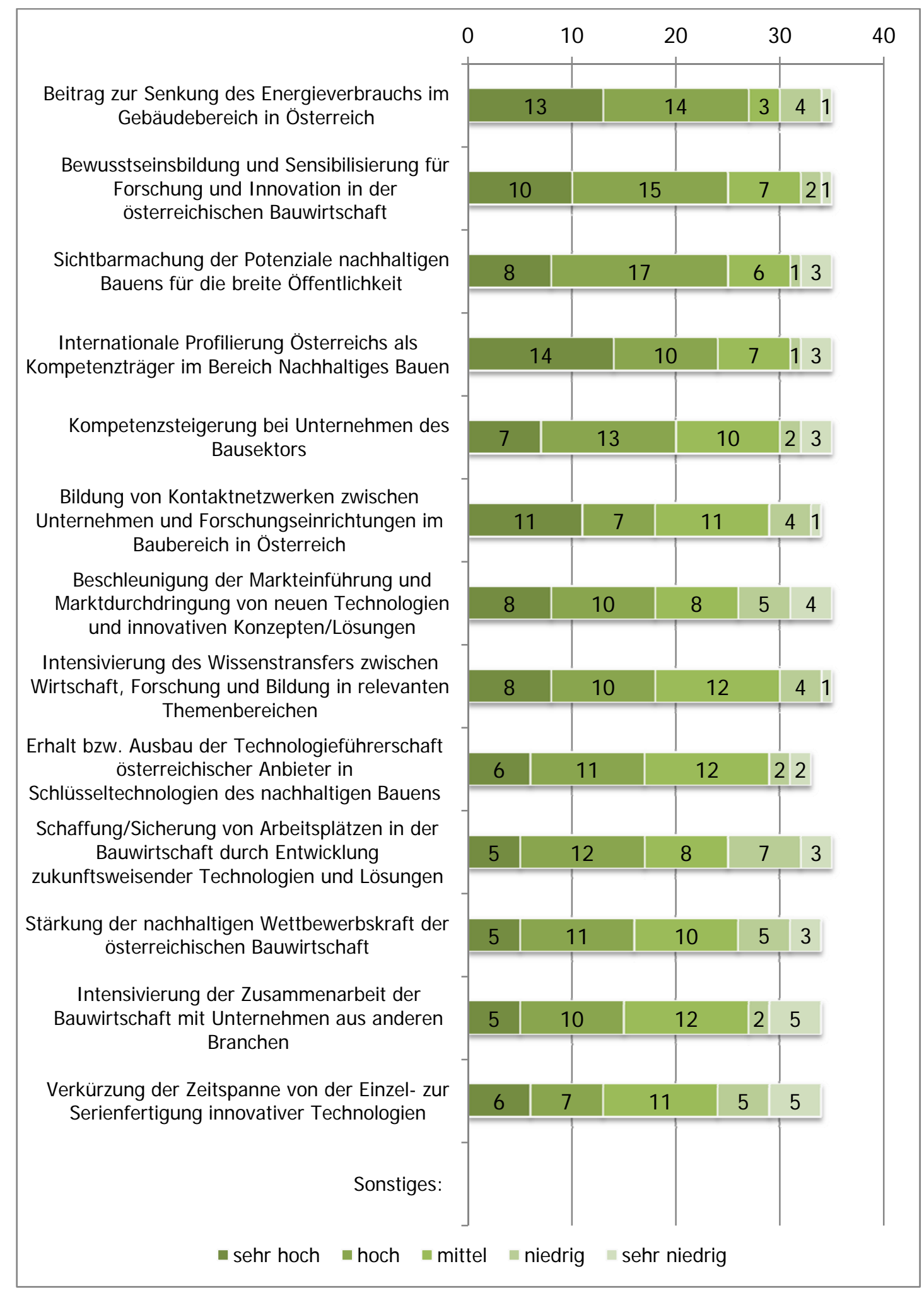

Quelle: Onlinebefragung von 37 Unternehmen; $n=35$ 
Die Wirkungen des Programms Haus der Zukunft aus Sicht der Unternehmen liegen insbesondere in folgenden Bereichen, wobei - wie der o.a. Grafik zu entnehmen ist - auch die anderen Wirkungsdimensionen hoch eingeschätzt werden:

* Beitrag zur Senkung des Energieverbrauchs im Gebäudebereich in Österreich

* Internationale Positionierung Österreichs als Kompetenzträger im Bereich Nachhaltiges Bauen

* Bewusstseinsbildung und Sensibilisierung für Forschung und Innovation in der österreichischen Bauwirtschaft

Die Begleitmaßnahmen - allen voran Informationsveranstaltungen, Workshops, Beratungsangebote, Kooperationsveranstaltungen - werden von den Unternehmen mehrheitlich als sehr wichtig bzw. wichtig angesehen.

\section{Abbildung 31: Wie wichtig waren nach I hrer Meinung die begleitenden Angebote des Programms Haus der Zukunft?}

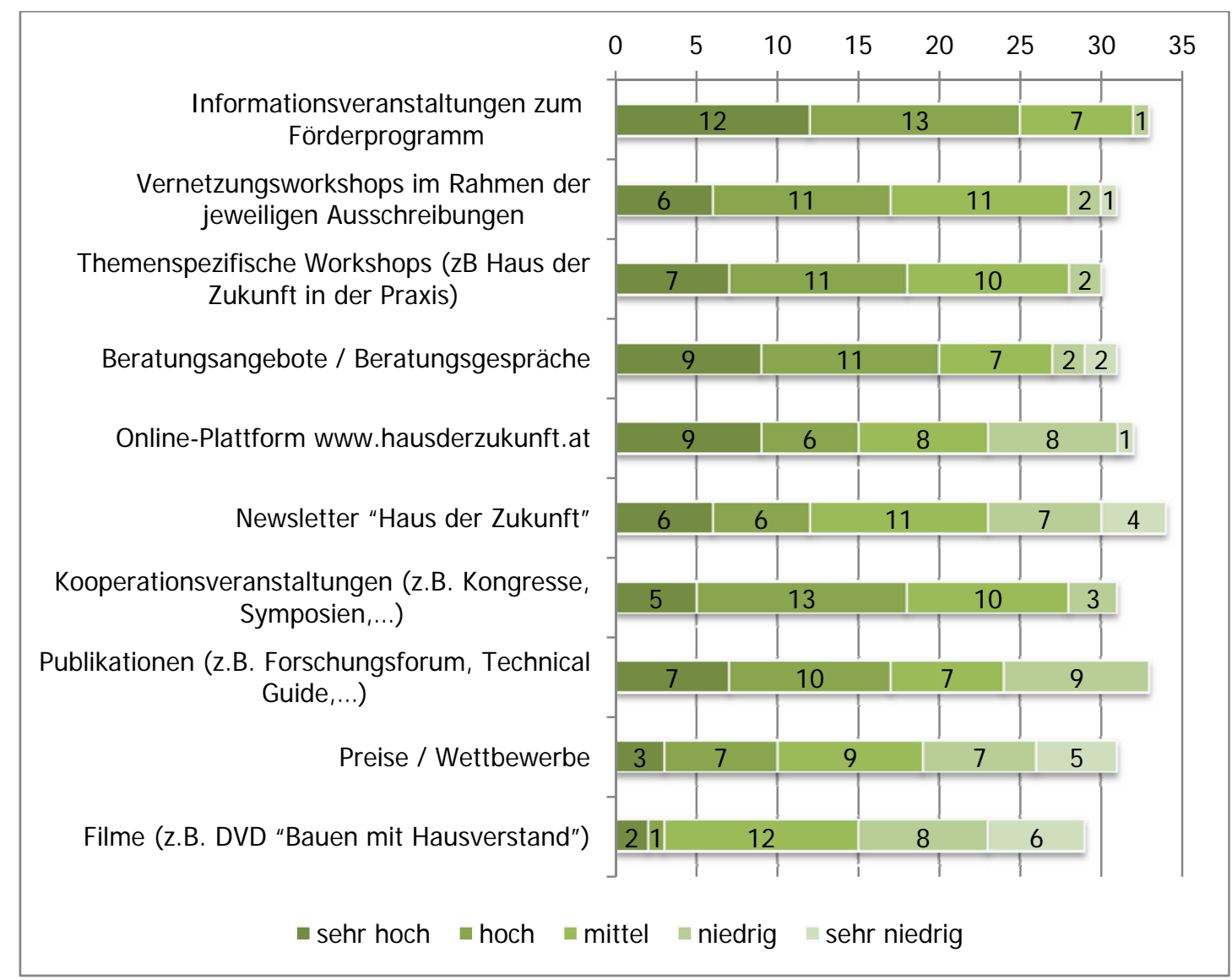

Quelle: Onlinebefragung von 37 Unternehmen; $n=34$ 
Abbildung 32: Wie relevant sind folgende Themenfelder für die künftigen Forschungsund I nnovationsaktivitäten? Bitte geben Sie auf der Skala die Relevanz dieser Themenfelder für I hr Unternehmen und für den Forschungs- und Wirtschaftsstandort Österreich insgesamt an!

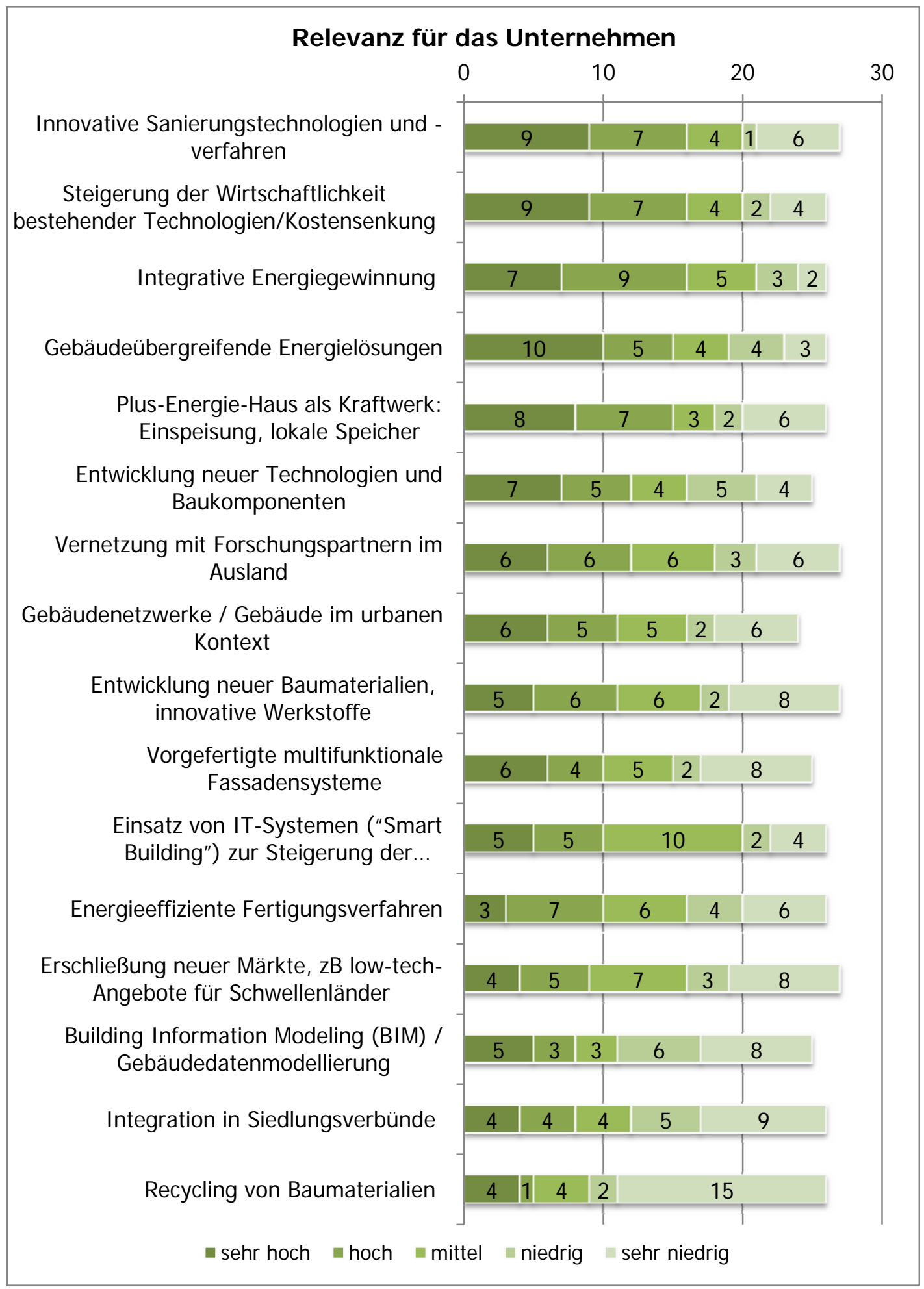

Quelle: Onlinebefragung von 37 Unternehmen; $n=27$ 
Mehr als $3 / 4$ der Unternehmen wollen auch in Zukunft Forschung und Innovation betreiben. Die wichtigsten Themenfelder für künftige Forschungs- und Innovationsaktivitäten im Unternehmen sind gebäudeübergreifende Energielösungen, innovative Sanierungstechnologien und -verfahren, die Steigerung der Wirtschaftlichkeit bestehender Technologien bzw. Kostensenkung, das PlusEnergie-Haus als Kraftwerk (Einspeisung, lokale Speicher) sowie integrative Energiegewinnung.

Geplant sind dazu insbesondere die Durchführung von weiteren geförderten Forschungsprojekten und Kooperationen mit Know-how-Trägern. Für die Umsetzung dieser Forschungsvorhaben sind in erster Linie Finanzmittel für Forschung und Innovation erforderlich, weiters die Vernetzung mit Partnern und qualifizierte Mitarbeiter. Das Spektrum an genannten konkreten Ideen, wie Forschung und Innovation im Bereich Nachhaltiges Bauen forciert werden könnten, ist sehr breit und reicht von forschungspolitischen Faktoren über die Bau- und Raumordnung bis hin zur allgemeinen Bewusstseinsbildung.

Abbildung 33: Was ist erforderlich, damit Sie diese Forschungs- und I nnovationsaktivitäten erfolgreich umsetzen können? (Mehrfachnennungen möglich)

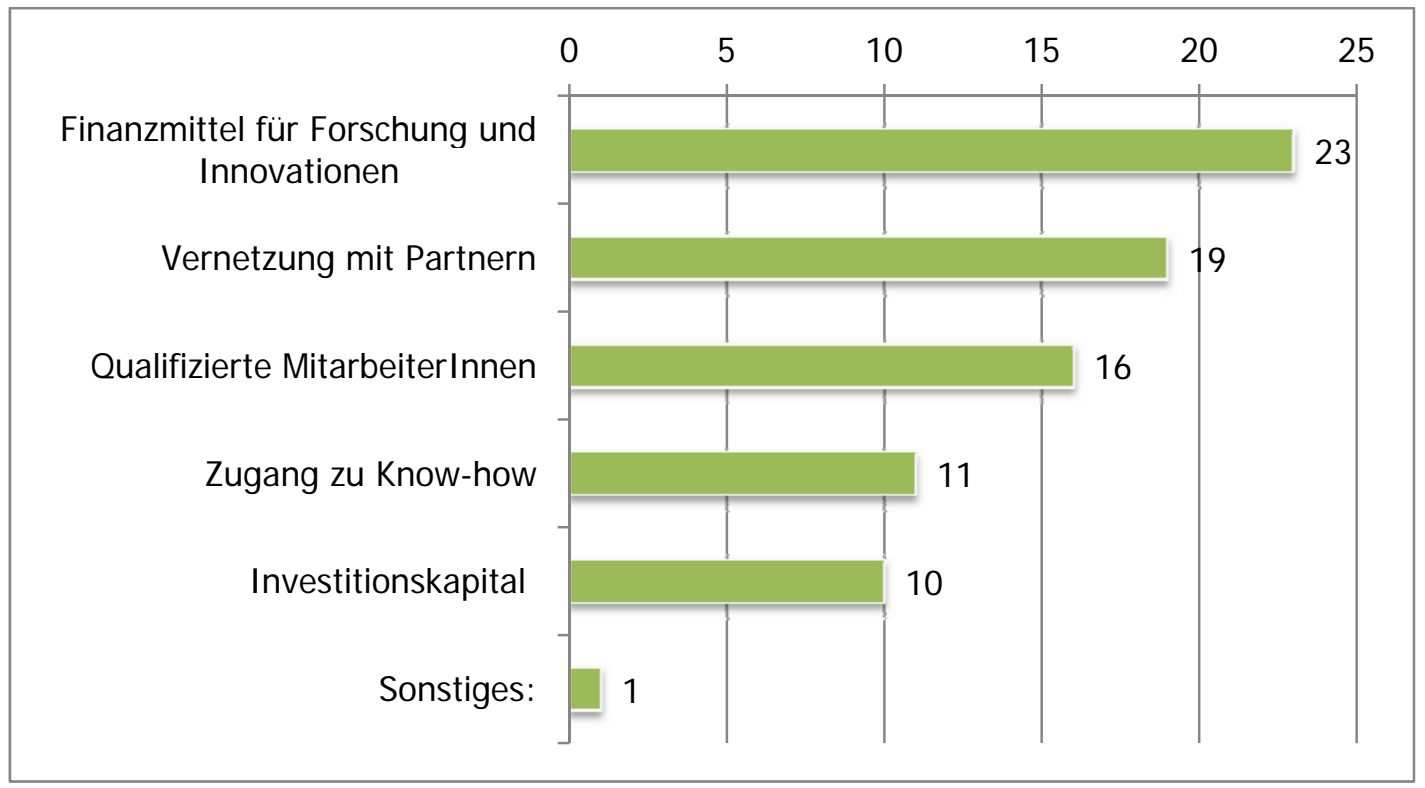

Quelle: Onlinebefragung von 37 Unternehmen; $n=26$ 


\subsubsection{Forschungseinrichtungen}

Weiters haben sich $\mathbf{4 0}$ Forschungseinrichtungen an der Onlinebefragung beteiligt. Der Fragebogen folgte derselben Struktur wie jener für Unternehmen, ging aber punktuell auf die Spezifika von Forschungseinrichtungen ein. 24 der 40 teilnehmenden Institutionen sind außeruniversitäre Forschungseinrichtungen, 13 sind Einrichtungen an einer öffentlichen Universität. 3 Befragte machten keine Angabe zum Organisationstyp. 16 Einrichtungen sind in Wien ansässig, 8 in der Steiermark und 5 in Oberösterreich.

Die an der Befragung teilnehmenden Forschungseinrichtungen haben an allen Haus der Zukunft-Ausschreibungen partizipiert. Die meisten Teilnahmen fanden in der ersten Ausschreibung der zweiten Programmphase statt, bei der u.a. die acht Leitprojekte gefördert wurden, an denen fünf der 40 Forschungseinrichtungen beteiligt waren.

Die Projekte von Forschungseinrichtungen waren meist in der Grundlagenforschung bzw. im Bereich der Begleitmaßnahmen sowie der Entwicklung innovativer Bau- und Sanierungskonzepte angesiedelt.

Abbildung 34: An welcher bzw. welchen Ausschreibung(en) des Förderprogramms Haus der Zukunft haben Sie teilgenommen? (Mehrfachnennungen möglich)

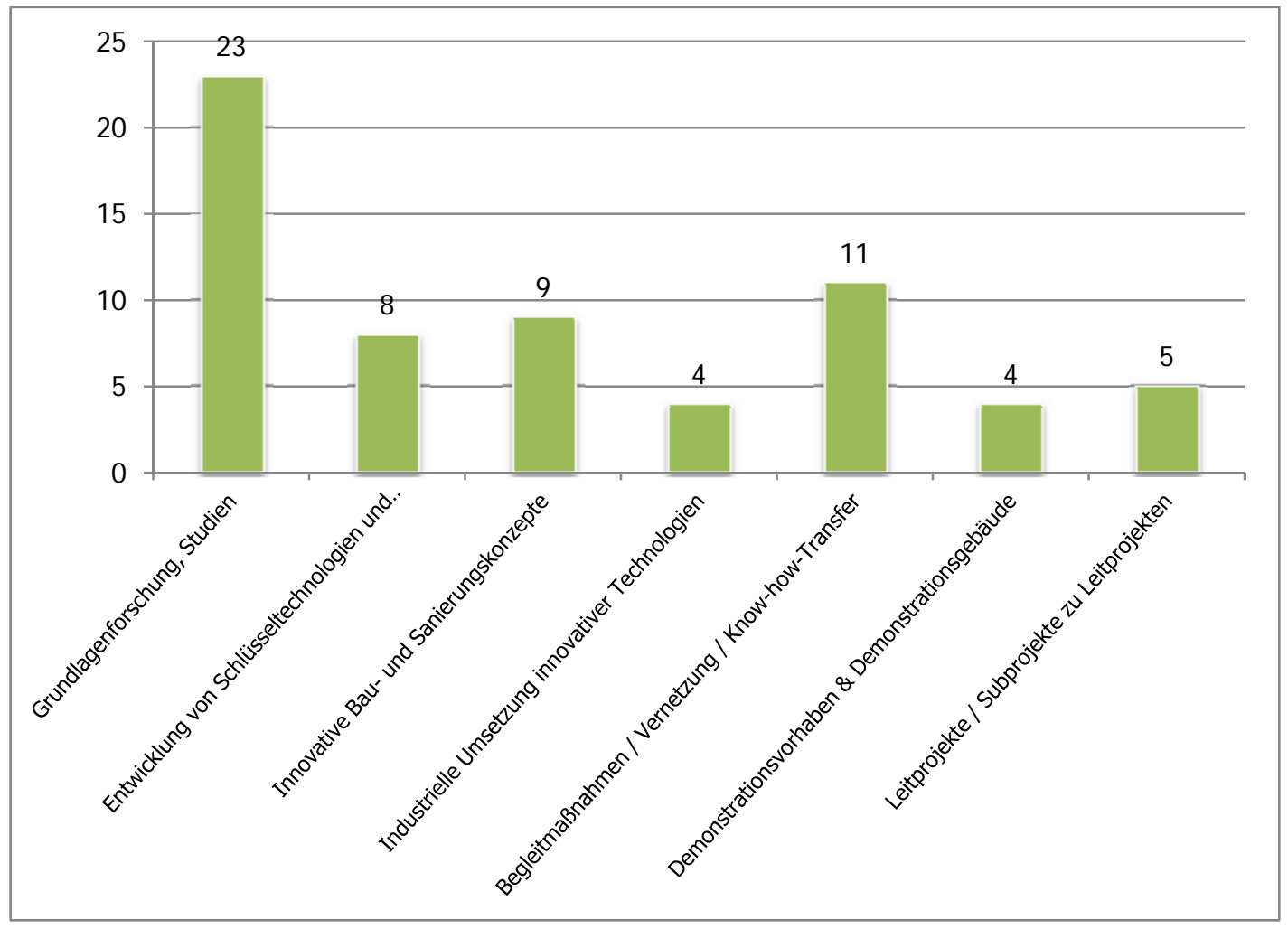

Quelle: Onlinebefragung von 40 Forschungseinrichtungen; $n=38$ 
Mehr als die Hälfte der Forschungseinrichtungen hätte ohne die Förderung nicht an dem Thema forschen können. 15 Einrichtungen hätten zwar das Thema behandelt, aber mit deutlich reduziertem Aufwand. Lediglich eine Forschungseinrichtung hätte auch ohne Haus der Zukunft-Förderung das Projekt umgesetzt. Der Mehrwert des Förderungsprogramms für die Forschungslandschaft im Themenfeld Nachhaltiges Bauen wird hieraus deutlich ersichtlich.

Abbildung 35: Hätte I hre Einrichtung auch ohne die Förderung an diesem Thema geforscht?

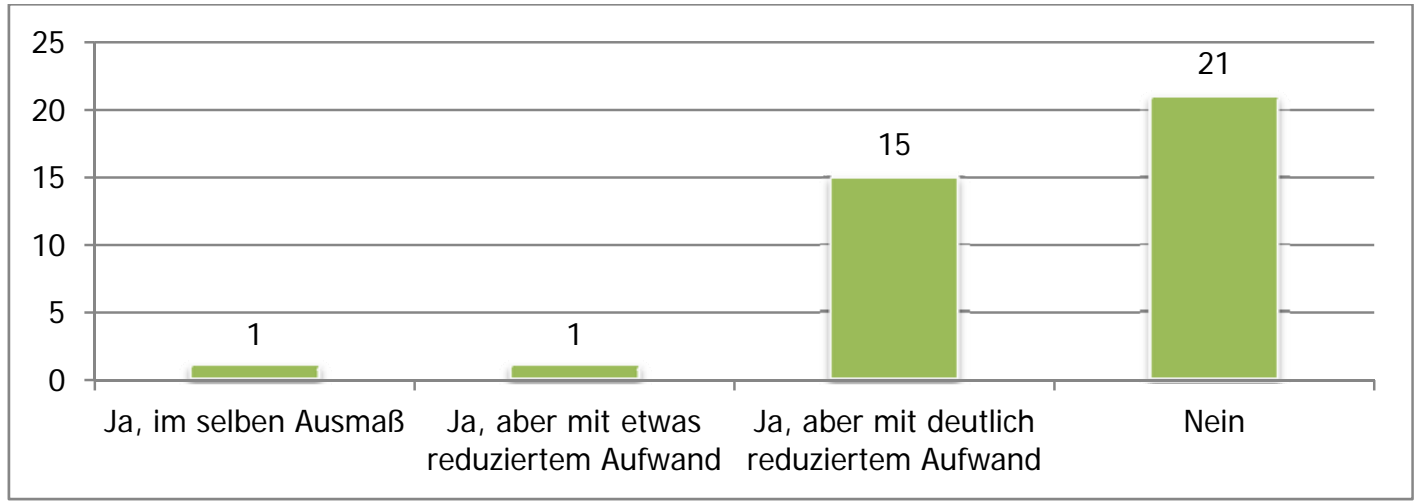

Quelle: Onlinebefragung von 40 Forschungseinrichtungen; $n=38$

Die höchsten unmittelbaren Wirkungen hatten die Projekte in Bezug auf die Finanzierung von wissenschaftlichem Personal, Impulse für wissenschaftliche Publikationen, Steigerung der Sichtbarkeit in der Fachcommunity sowie Steigerung der internationalen Sichtbarkeit der Forschungseinrichtung. Eine Einrichtung gab unter „Sonstiges" an, damit eine Entscheidungsgrundlage für weitere F\&E-Aktivitäten entwickelt zu haben.

Ein Viertel der befragten Einrichtungen hat im Zuge des Projektes ein neues Produkt, eine neue Technologie oder ein neues Dienstleistungsangebot entwickelt. Das Spektrum an Neuentwicklungen von Forschungseinrichtungen umfasst sowohl eine neue Technologie ( $z B$ polymere Funktionsmaterialien) wie auch ein innovatives Gebäudekonzept (zB Konzept für ein bionisches Haus) und neuartige Dienstleistungen in Zusammenhang mit nachhaltigen Gebäuden (zB Energiemodellierung). 
Abbildung 36: Welche Auswirkung hatte das Projekt unmittelbar auf I hre Einrichtung? Bitte geben Sie auf der Skala an, was durch das Projekt in I hrem Unternehmen bewirkt wurde!

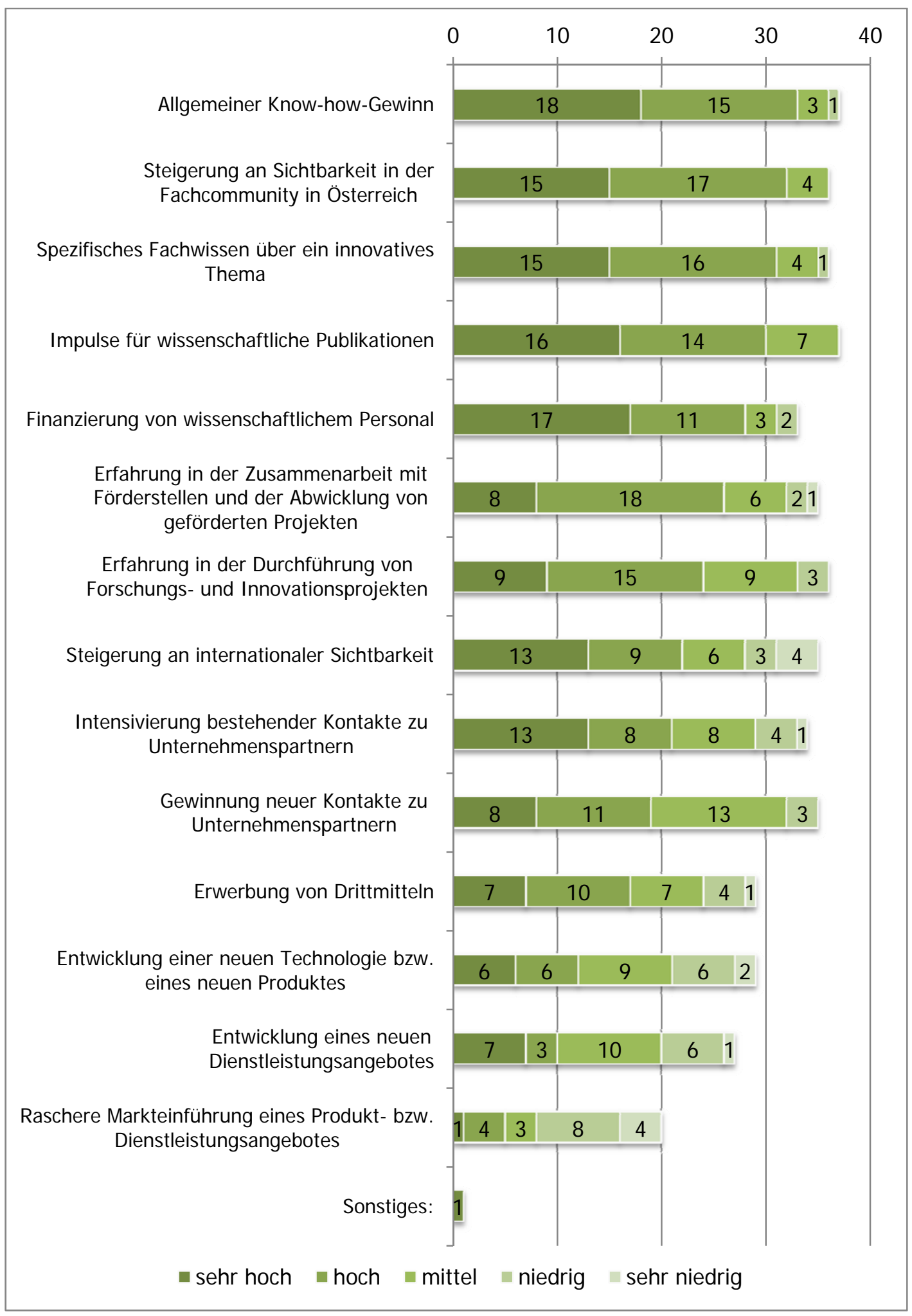

Quelle: Onlinebefragung von 40 Forschungseinrichtungen; $n=38$ 
5 von 6 Einrichtungen geben an, dass das Projekt über die Laufzeit hinweg nachhaltige Wirkung auf die Forschungseinrichtung hatte. Diese liegt vorrangig in drei Bereichen: Durch das Projekt konnte wissenschaftliches Personal aufgenommen bzw. gehalten werden. Weiters haben die Projekte maßgeblich zur Herausbildung von Forschungsschwerpunkten beitragen. Nicht zuletzt haben die Haus der Zukunft-Projekte auch zur Schärfung und Verbreitung des Profils der wissenschaftlichen Einrichtungen beigetragen, die sich etwa in Nachfolgeprojekten im Rahmen von europäischen und internationalen Programmen niederschlagen.

Bis auf wenige Ausnahmen wurde auch nach Abschluss des Projektes weiter an dem Thema geforscht. Der Großteil hat dies in Form eines nachfolgenden Forschungs- und Innovationsprojektes aus einem anderen Programm getan. 7 der 40 Forschungseinrichtungen haben ein weiteres Haus der Zukunft-Projekt angeschlossen. Unter "Sonstiges“ wurden u.a. Postdoc-Forschungsstelle oder Literaturstudium angeführt.

Abbildung 37: Haben Sie nach Abschluss des geförderten Projektes weiter an dem Thema gearbeitet? Wenn ja, in welcher Form?

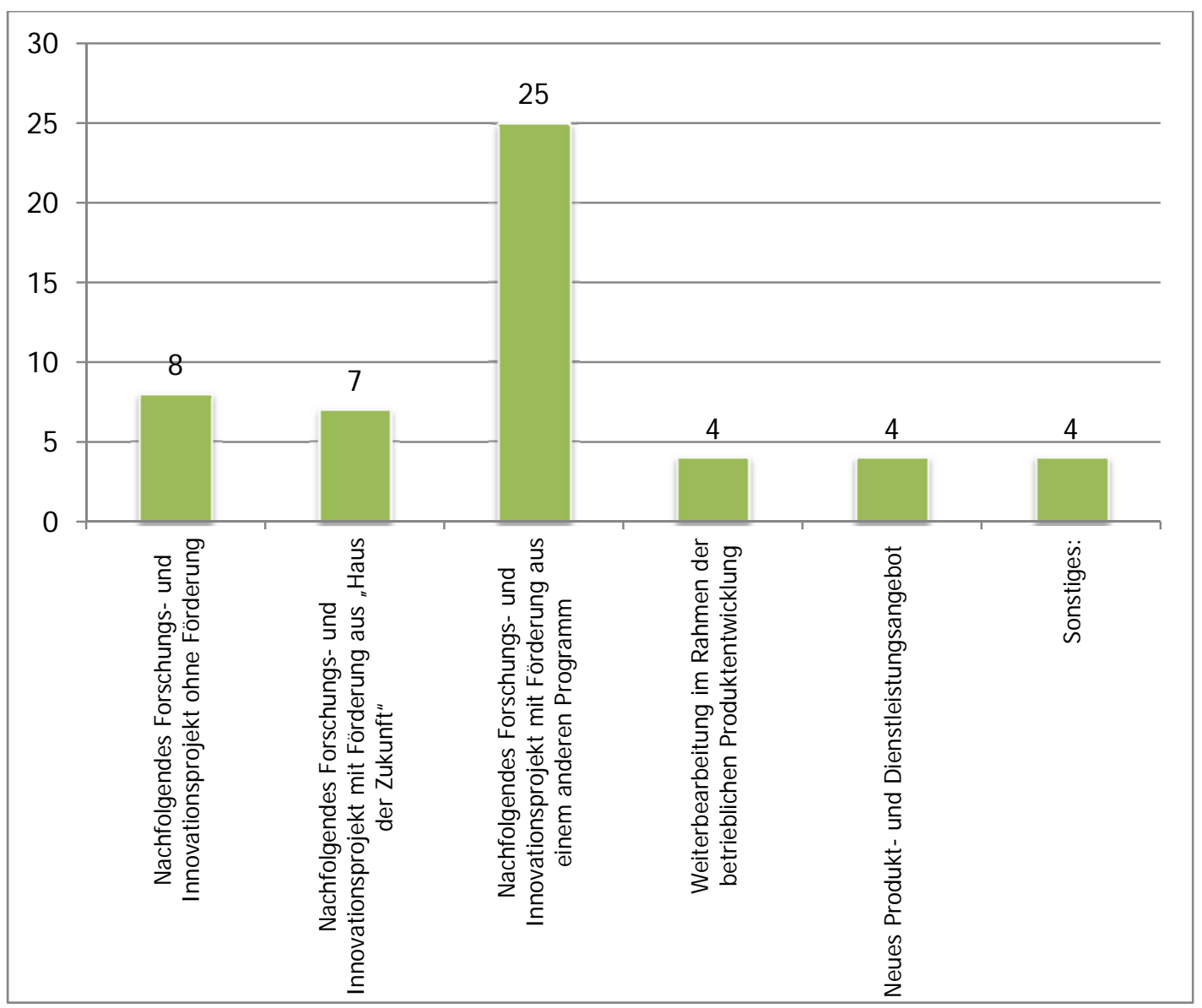

Quelle: Onlinebefragung von 40 Forschungseinrichtungen; $n=31$ 
Das Programm Haus der Zukunft hatte nach Einschätzung der Forschungseinrichtungen eine sehr hohe Wirkung in Bezug auf Bewusstseinsbildung und Sensibilisierung für die Thematik, die internationale Profilierung Österreichs als Kompetenzträger im Bereich Nachhaltiges Bauen, die Intensivierung des Wissenstransfers Wirtschaft/Wissenschaft/Öffentlicher Sektor sowie die Bildung von Kontaktnetzwerken zwischen Unternehmen und Forschungseinrichtungen in Österreich.

Abbildung 38: Was konnte nach I hrer Wahrnehmung durch das Programm Haus der Zukunft insgesamt in Österreich bewirkt werden? Bitte geben Sie auf der Skala an, wie hoch Sie die Wirkung einschätzen!

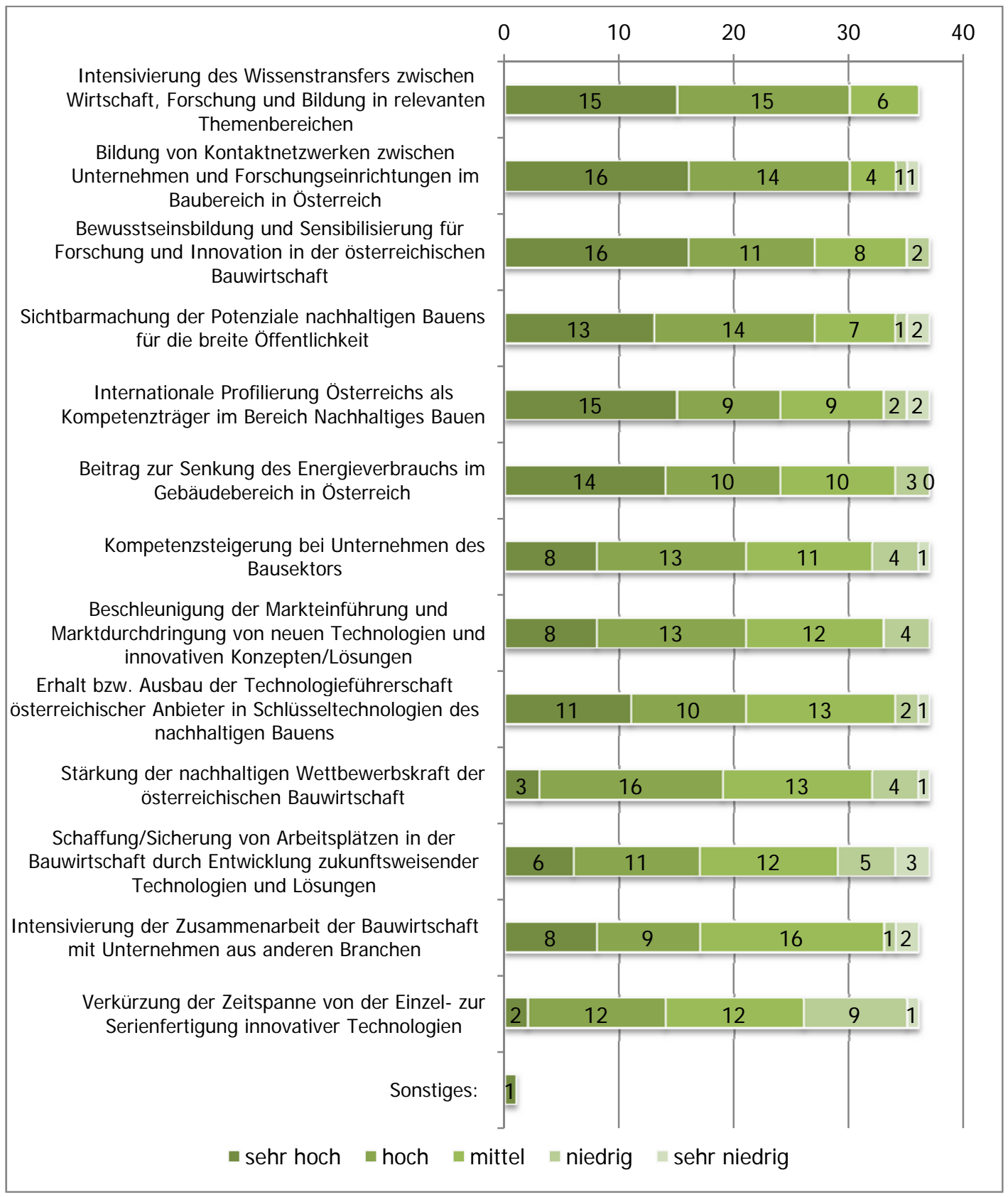

Quelle: Onlinebefragung von 40 Forschungseinrichtungen; $n=37$ 
Die Begleitmaßnahmen waren aus Sicht der Forschungseinrichtungen von zentraler Bedeutung. Besonders hoch eingeschätzt wird die Bedeutung von Publikationen, der Online-Plattform und die themenspezifischen Workshops.

Abbildung 39: Wie wichtig waren nach I hrer Meinung die begleitenden Angebote des Programms Haus der Zukunft?

\begin{tabular}{|c|c|c|c|c|c|c|c|c|c|}
\hline & 0 & 10 & 15 & 20 & & 3 & 0 & 35 & 40 \\
\hline Online-Plattform www. hausderzukunft. at & & 12 & & 13 & & 5 & 31 & & \\
\hline Publikationen (z.B. Forschungsforum, & & 13 & & 11 & & 6 & 11 & & \\
\hline Themenspezifische Workshops ( $z B$ Haus & & 12 & ( & & & 9 & 3 & & \\
\hline $\begin{array}{c}\text { Kooperationsveranstaltungen (z.B. } \\
\text { Konaresse Symposien }\end{array}$ & 3 & $1 \varepsilon$ & & & 7 & 2 & & & \\
\hline Beratungsangebote / Beratungsgespräche & 5 & 14 & & & 7 & 31 & & & \\
\hline $\begin{array}{c}\text { Intormationsveranstaltungen zum } \\
\text { Förderprogramm }\end{array}$ & 3 & 13 & & & 15 & & 3 & 2 & \\
\hline $\begin{array}{l}\text { Vernetzungsworkshops im Rahmen der } \\
\text { jeweiligen Ausschreibungen }\end{array}$ & 5 & 11 & & & 13 & & 42 & & \\
\hline Newsletter "Haus der Zukunft" & 6 & 10 & & & 1 & 4 & 2 & & \\
\hline Preise / Wettbewerbe & 4 & 10 & & 9 & & 61 & & & \\
\hline $\begin{array}{c}\text { Filme (z.B. DVD "Bauen mit } \\
\text { Hausverstand") }\end{array}$ & 2 & 6 & 11 & & 3 & & & & \\
\hline - sehr hoch $\quad$ hoch $\square$ mit & & niedrig & & & & & & & \\
\hline
\end{tabular}

Quelle: Onlinebefragung von 40 Forschungseinrichtungen; $n=36$

35 der 40 befragten Forschungseinrichtungen planen, auch in Zukunft Forschungs- und Innovationsaktivitäten umzusetzen. Jene 5 Einrichtungen, die keine weiteren Projekte planen, haben als Gründe dafür angegeben, sich derzeit auf die Grundlagenforschung zu fokussieren, aktuell keine relevanten Themen zu identifizieren sowie ein zu niedriges Niveau der Forschungsprojekte im internationalen Vergleich zu haben. Für die anderen Befragten sind wichtige Forschungs- und I nnovationthemen der Zukunft insb. die Vernetzung mit Forschungspartnern im Ausland, gebäudeübergreifende Energielösungen, Gebäudenetzwerke und Einspeisung/lokale Speicher. 
Abbilldung 40: Wie relevant sind folgende Themenfelder für die künftigen Forschungsund I nnovationsaktivitäten? Bitte geben Sie auf der Skala die Relevanz dieser Themenfelder für I hr Unternehmen und für den Forschungs- und Wirtschaftsstandort Österreich insgesamt an!

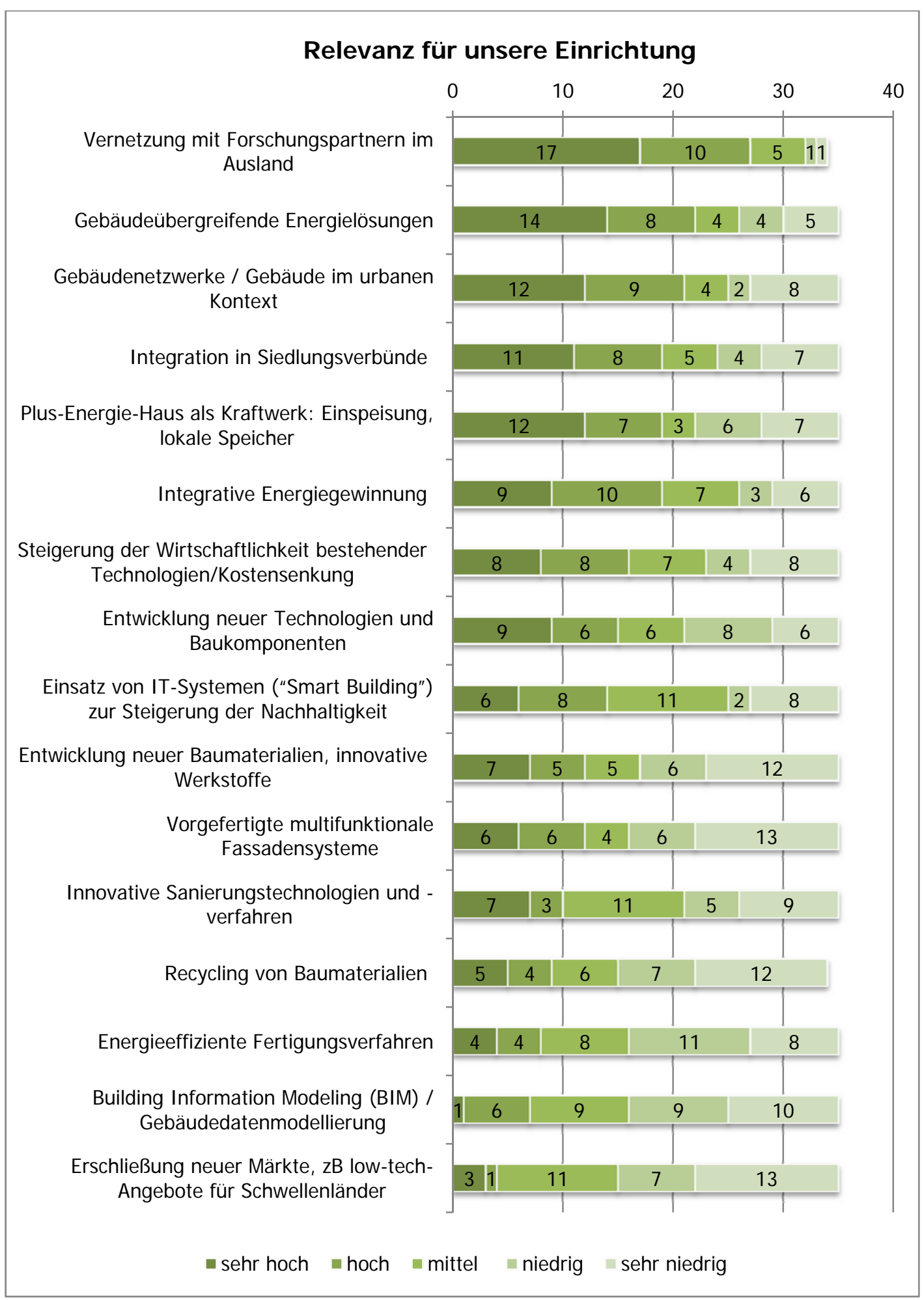

Quelle: Onlinebefragung von 40 Forschungseinrichtungen; $n=35$ 
Eine Reihe von Themen ist für die befragten Forschungseinrichtungen in Zukunft von Interesse. Das Spektrum reicht von integrativer Planung über neue Materialien, Energiespeichertechnologien und Sanierung bis hin zu „Smart Building“ und dem Gebäude im urbanen Verbund. Das breite Themenspektrum - eine vollständige Auflistung der genannten Themenfelder ist im Anhang zum Evaluierungsbericht angeführt - spiegelt nicht nur die inhaltliche Breite und Tiefe von Nachhaltigem Bauen wider, sondern auch die Heterogenität und den hohen Grad an Spezialisierung, in der österreichischen Forschungscommunity.

Die Auseinandersetzung mit diesen Themengebieten wird voraussichtlich in Form von geförderten Forschungsprojekten mit Unternehmen sowie Kooperationen mit anderen wissenschaftlichen Einrichtungen erfolgen - jeweils $3 / 4$ der befragten Forschungseinrichtungen planen diesbezügliche Aktivitäten. Unter "Sonstiges" wurde etwa ein EU-Projekt genannt oder die Ausrichtung einer Fachveranstaltung.

Abbildung 41: In welcher Form planen Sie, sich mit diesen Themenfeldern auseinanderzusetzen?

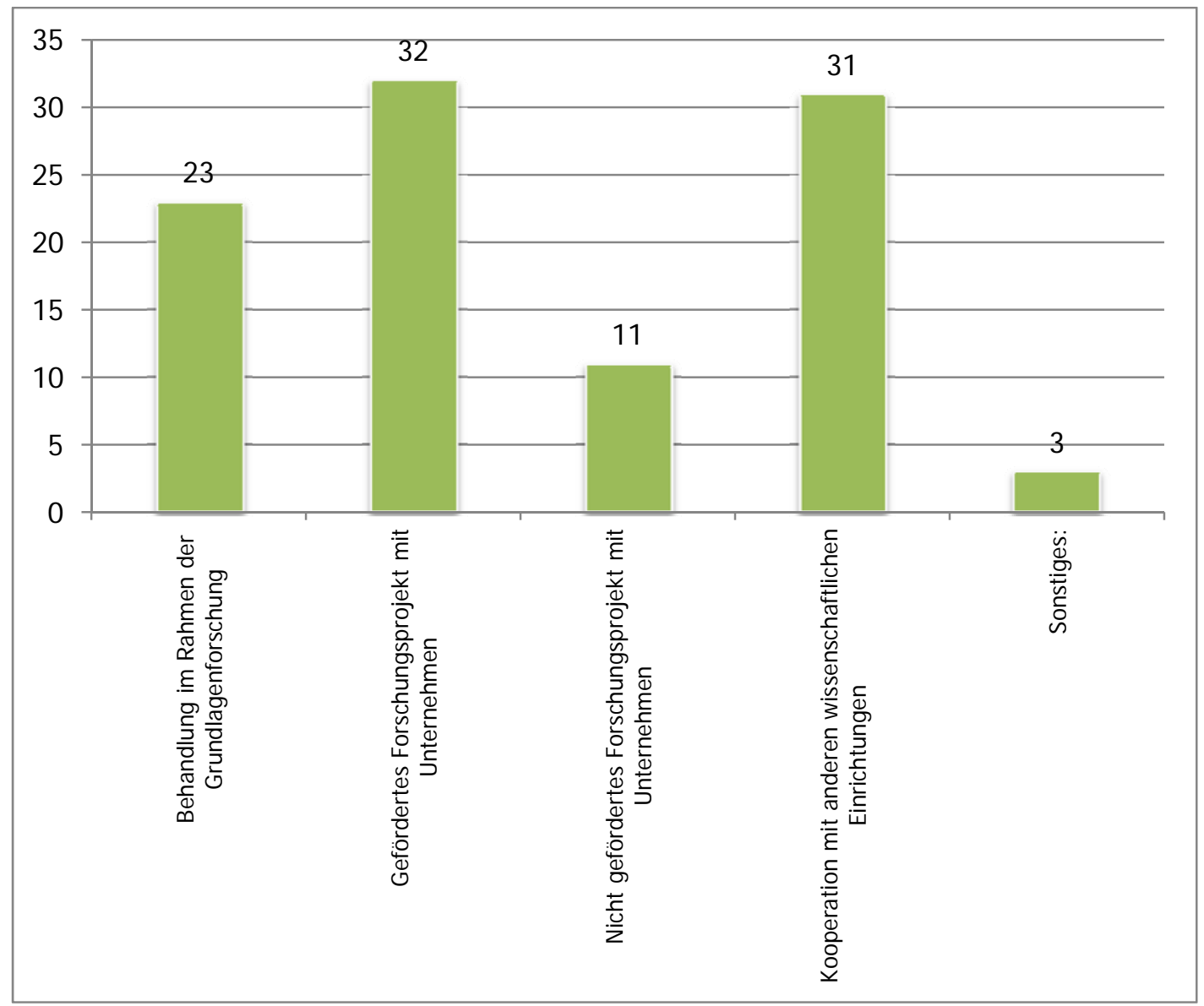

Quelle: Onlinebefragung von 40 Forschungseinrichtungen; $n=35$ 
Damit es zur Umsetzung der geplanten Forschungs- und Innovationsaktivitäten kommen kann, sind unterschiedliche Unterstützungsmaßnahmen erforderlich. Am wichtigsten ist den befragten Einrichtungen die Verfügbarkeit von Fördermitteln für Forschungsprojekte, aber auch qualifizierte Mitarbeiter, die Vernetzung mit Unternehmenspartnern und anderen wissenschaftlichen Einrichtungen sind von großer Bedeutung. Nur für rund ein Drittel der Befragten ist Investitionskapital für Forschungsinfrastrukturen erforderlich.

Abbildung 42: Was ist erforderlich, damit Sie diese Forschungs- und Innovationsaktivitäten erfolgreich umsetzen können?

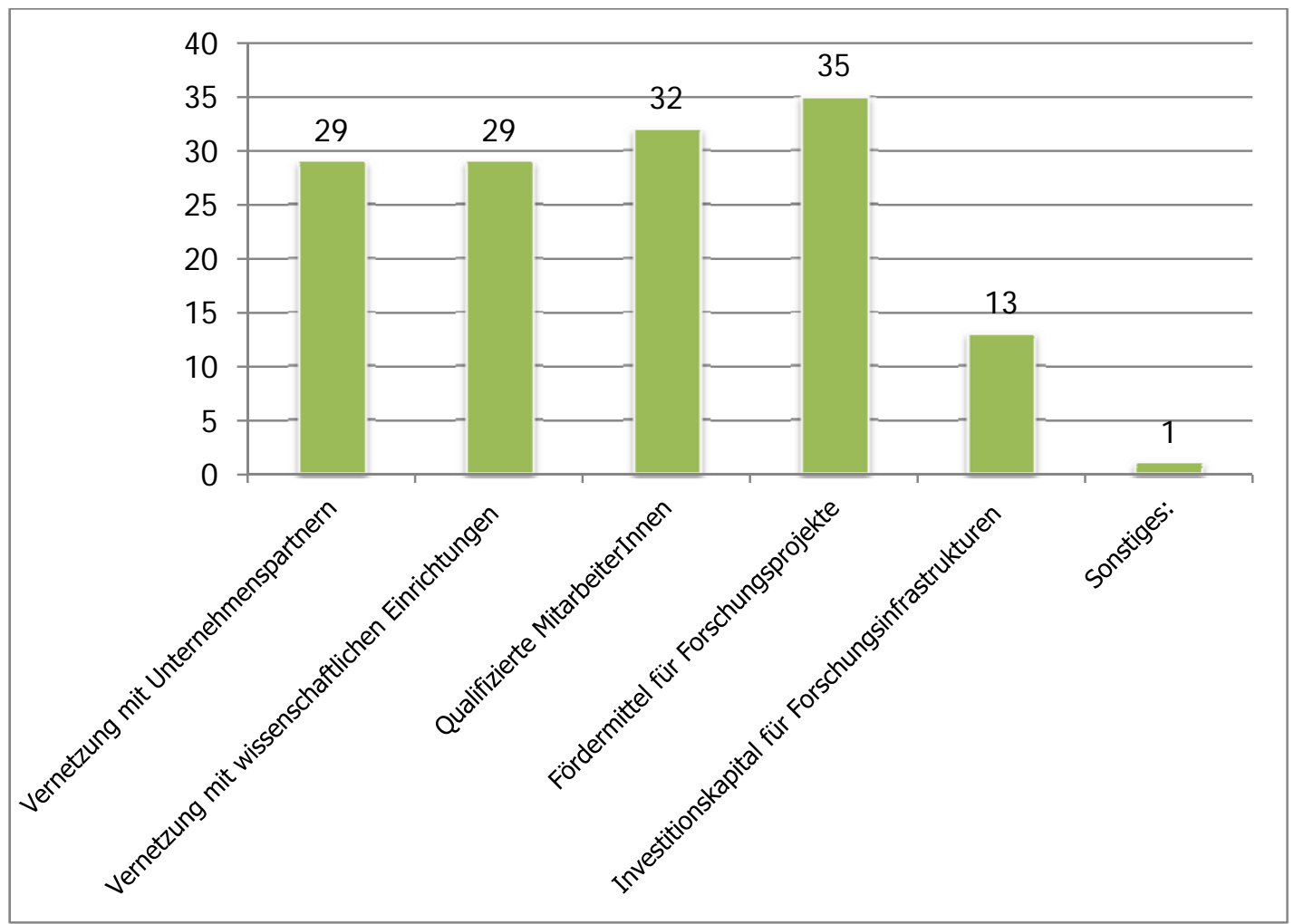

Quelle: Onlinebefragung von 40 Forschungseinrichtungen; $n=35$

Abschließend wurden die Forschungseinrichtungen nach konkreten I deen und Anregungen zur Forcierung von Forschung und Innovation im Bereich Nachhaltiges Bauen in Österreich gefragt. Neben der Forschungsförderung wurden dabei auch die Ausbildung/Qualifizierung in der Bauwirtschaft, die Berücksichtigung sozialer Aspekte in der Bauforschung, die Sensibilisierung der Öffentlichkeit bzw. der Bauherrn für die Thematik, die Zugänglichkeit von Demonstrationsprojekten, eine stärkere Berücksichtigung von Nachhaltigkeitskriterien in der Bauordnung/Wohnbauförderung, eine stärkere Unterstützung bei der Marktüberführung innovativer Technologien uvam. mehr angesprochen. 


\subsubsection{Sonstige Einrichtungen}

Die dritte Gruppe der Befragten stellten sonstige Einrichtungen dar. Von den sieben teilnehmenden Akteuren sind drei als öffentliche Einrichtungen zu kategorisieren, zwei als Vereine und zwei als weitere Einrichtungen ohne nähere Angabe. Jeweils zwei Einrichtungen sind in Wien und Tirol ansässig, jeweils eine in Vorarlberg und Oberösterreich. Aufgrund dieser geringen Anzahl an Teilnehmern sind prozentuale Auswertungen wenig aussagekräftig - nichtsdestotrotz eröffnet sich durch die Perspektive dieser sonstigen Einrichtungen eine wertvolle Ergänzung.

Die sonstigen Einrichtungen haben sich an verschiedenen Haus der ZukunftAusschreibungen beteiligt. Jeweils vier Einrichtungen haben sich an den Projektarten "Innovative Bau- und Sanierungskonzepte“ sowie "Begleitmaßnahmen“ beteiligt.

Vier Einrichtungen haben angegeben, dass sie ohne die Förderung zwar das Projekt dennoch realisiert hätten, allerdings mit deutlich reduziertem Aufwand. Eine Einrichtung hat deutlich gemacht, dass sie ohne die Förderung das Projekt nicht umgesetzt hätte.

Die unmittelbaren Wirkungen, die durch das Projekt für die Einrichtung generiert worden sind, liegen in erster Linie in der Gewinnung von spezifischem Fachwissen, Intensivierung bestehender und Gewinnung neuer Kontakte zu Partnerorganisationen sowie der Steigerung an Sichtbarkeit in der Fachcommunity.

Unisono gaben die sonstigen Einrichtungen an, dass das Projekt über die Laufzeit hinaus nachhaltige Wirkungen auf die Einrichtung hatte. Durch Haus der Zukunft konnte bei den sonstigen Einrichtungen Know-how aufgebaut werden, Verbindungen zu Kompetenzpartnern aufgebaut bzw. vertieft werden und Nachfolgeprojekte vorbereitet werden. Dementsprechend haben alle Einrichtungen weiter an dem Thema geforscht.

2/3 der Einrichtungen haben ein neues Produkt, eine neue Technologie oder ein neues Dienstleistungsangebot aus dem Projekt entwickelt. Dabei handelte es sich um Effizienztechnologien für historische Gebäude, die Erweiterung von Beratungsangeboten und die Erstellung eines Leitfadens für Kommunen für die Einführung von Technologien für „Anergie“-Netzwerke. ${ }^{6}$

Haus der Zukunft in seiner Gesamtheit hat aus Sicht der sonstigen Einrichtungen maßgeblich zur Bewusstseinsbildung und Sensibilisierung für Forschung und Inno-

6 Als Anergie wird der Bestandteil einer Energie bezeichnet, der in einem Prozess keine Arbeit verrichten kann. Die Anergie gibt an, wie viel Energie maximal gewonnen werden könnte, wenn ein System, dessen Energie durch einen Prozess bereits erschöpfend genutzt worden ist, durch einen idealen Prozess in ein neues Gleichgewicht mit einer absolut kalten Umgebung gebracht würde. 
vation in der österreichischen Bauwirtschaft, der internationalen Profilierung Österreichs, der Senkung des Energieverbrauchs im Gebäudebereich, der Sichtbarmachung der Potenziale Nachhaltigen Bauens und der Intensivierung des Wissenstransfers beigetragen.

Unter den Begleitmaßnahmen schätzen die sonstigen Einrichtungen in erster Linie Kooperationsveranstaltungen und Publikationen als sehr hilfreich ein. Weiters wurden die Vernetzungsworkshops im Rahmen der jeweiligen Ausschreibungen, themenspezifische Workshops, Beratungsangebote und Medien intensiv genutzt.

Abbildung 43: Wie wichtig waren nach I hrer Meinung die begleitenden Angebote des Programms „Haus der Zukunft“?

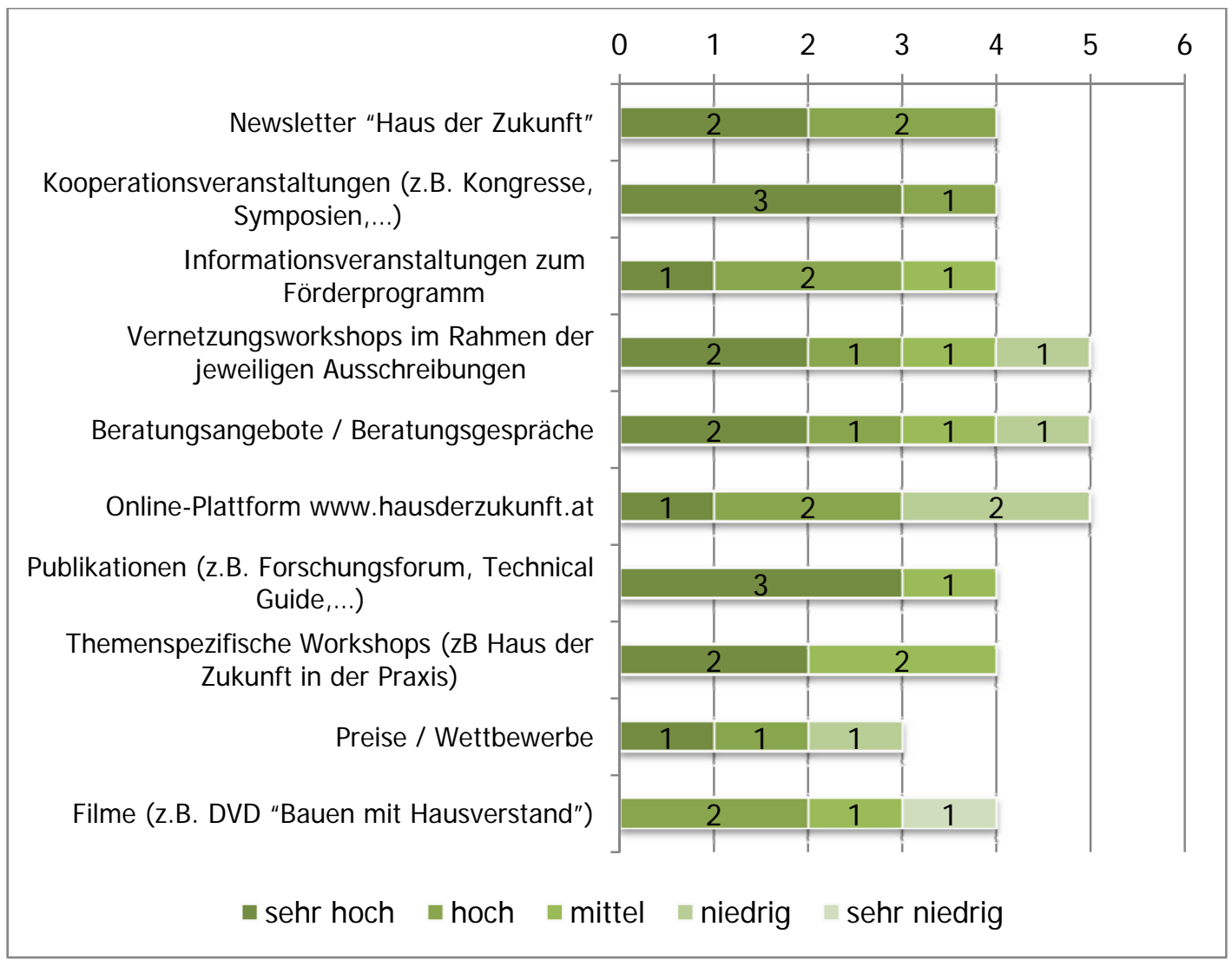

Quelle: Onlinebefragung von 7 sonstigen Einrichtungen; $n=5$

5 der 7 sonstigen Einrichtungen beabsichtigen auch in Zukunft Forschungs- und Innovationsaktivitäten im Bereich Nachhaltiges Bauen umzusetzen. Die wichtigsten Themenfelder sind dabei "Innovative Sanierungstechnologien und -verfahren", „Plus-Energie-Haus als Kraftwerk: Einspeisung, lokale Speicher" und "Gebäudeübergreifende Energielösungen“. Darüber hinaus sind auch die Entwicklung neuer Baumaterialen und innovativer Werkstoffe, integrative Energiegewinnung und die Steigerung der Wirtschaftlichkeit bestehender Technologien bzw. Kostensenkung von Interesse. 


\subsection{Interviews mit Schlüsselpersonen}

In Ergänzung zur Onlinebefragung wurden mit ausgewählten Personen vertiefende Interviews geführt. Dabei wurden drei Gruppen abgebildet:

* Ausgewählte Projektträger, die aufgrund der durchgeführten Projekte oder ihrer Funktion einen besonders tiefen Einblick in die Programmumsetzung und - wirkungen haben

* Stakeholder der österreichischen Forschungs- und Wirtschaftscommunity im Themenfeld Nachhaltiges Bauen

* Internationale Experten, die Haus der Zukunft aus einer Außensicht einschätzen können

Für die Gespräche wurden Gesprächsleitfäden erstellt, welche sich ebenso wie die Listen der Gesprächspartner im Anhang zum Evaluierungsbericht finden. Nachfolgend werden die Kernergebnisse der Interviews in den drei Gruppen zusammengefasst.

\subsubsection{I nterviews mit ausgewählten Projektträgern}

Die 8 im Zuge der Evaluierung interviewten Projektträger sind allesamt dem Forschungsbereich zuzuordnen. 6 davon sind an einer öffentlichen Universität tätig, 2 in der außeruniversitären Forschung.

Die befragten Personen waren zumeist an mehreren Haus der ZukunftProjekten beteiligt, davon vielfach auch als Projektleiter. Einige der befragten Projektträger haben bereits vor oder zu Beginn von Haus der Zukunft im Themenfeld Nachhaltiges Bauen geforscht und so das Programm von Grund auf genutzt. Zum Teil wurden auch neue Forschungsthemen von den Forschern eingebracht die aktive Einladung zur Einbringung von Themenvorschlägen wird hoch geschätzt -, sodass die Entwicklungsgeschichte des Programms eng mit der Entwicklung des gesamten Forschungsbereiches in Österreich verwoben ist.

Das Programm im Allgemeinen und die Programmumsetzung im Besonderen wurde vom Großteil als sehr gut eingeschätzt. Insbesondere den Kontaktpersonen bei den involvierten Institutionen (BMVIT, FFG, aws, ÖGUT) wurde von der Mehrzahl der befragten Projektträger eine hoch fachliche Kompetenz und Kooperationsbereitschaft konstatiert.

Mehrfach wurde auch der Vergabeprozess erwähnt: Die Ausschreibung von Forschungsprojekten durch Calls war dazumals ein Novum und ist heute ein StandardProzedere. Die international besetzte Jury hat sich aus Sicht der Projektträger bewährt und zu einer hohen fachlichen Qualität der Projekte beigetragen. Bei einer Ablehnung des Förderungsansuchens wurde eine fachliche Begründung angeführt. 
Die Begleitmaßnahmen werden von den Projektträgern als sehr wertvoll eingeschätzt, insbesondere zur Information über die Ergebnisse von Projekten, Vernetzung mit anderen Akteuren, Verbreitung von neu gewonnenem Wissen etc. Als entbehrlich wurden Workshops und Veranstaltungen mit einem stets sehr ähnlichen Teilnehmerkreis wahrgenommen. Der „Zwang zur Dissemination“ mag zuweilen mit einem Aufwand verbunden sein, der für den einzelnen Forscher wenig unmittelbaren Mehrwert bringt, ist aber in Summe als Teil des "Erfolgsrezeptes" von Haus der Zukunft zu sehen.

Angeregt wurde hier, im Rahmen der Dissemination nicht nur erfolgreiche Projekte vor den Vorhang zu holen, sondern auch gescheiterte Projekte und die "lessons learned" in der Community zu verbreiten. Aus rein fachlicher Sicht ist die Erkenntnis über eine Technologie oder eine Gebäudelösung, die nicht funktioniert, ebenso wichtig wie die Erkenntnis über erfolgreiche Ansätze. Nachvollziehbar ist, dass Fördernehmer wenig Interesse daran haben, nicht erfolgreiche Projekte in der Fachcommunity und gegenüber den Förderungsinstitutionen besonders hervorzuheben.

Das Programm hatte für alle Einrichtungen einen wesentlichen und nachhaltigen Impact. Ein Programm wie Haus der Zukunft ermöglicht spezialisierten Forschungseinrichtungen in diesem Themenfeld die Möglichkeit, eine Reihe von fachlich interessanten Projekten zu attraktiven Förderkonditionen durchzuführen und somit Forschungsgelder zu lukrieren. Die lange Laufzeit des Programms wurde dabei besonders positiv unterstrichen, welche in Verbindung mit der stetigen Weiterentwicklung in Hinblick auf die Themenschwerpunkte und Instrumente einen kontinuierlichen Aufbau von Kompetenzen und Ressourcen in den Forschungseinrichtungen ermöglichte. Mehrfach berichteten die Forscher, dass sie von ihren ausländischen Kollegen regelrecht um ein Programm wie Haus der Zukunft „beneidet“ werden.

Viele Haus der Zukunft-Projekte wurden in Kooperation mit Unternehmen oder weiteren wissenschaftlichen Einrichtungen durchgeführt. Diese Kooperationen wären ohne Haus der Zukunft vielleicht auch zustande gekommen, jedoch nicht in jener Tiefe und in jenem Umfang. Mehrfach wurde von Kooperationen berichtet, die über die geförderten Projekte hinaus Bestand haben.

Haus der Zukunft ist für viele der befragten Einrichtungen zu einem „Türöffner“ für europäische und internationale Projekte geworden. Aufgrund der hohen internationalen Bekanntheit von Haus der Zukunft haben erfolgreiche nationale Projekte konkrete Anknüpfungsmöglichkeiten im Rahmen von europäischen und internationalen Förderprojekten ergeben. Ein Gesprächspartner bezeichnete Haus der Zukunft als "Gütesiegel für Forschung im Bereich Nachhaltiges Bauen“, mit dem sich ein Institut auf europäischer Ebene positionieren kann. Damit wird nicht nur ein Beitrag zur Internationalisierung der österreichischen Forschungslandschaft 
eleistet, sondern es werden auch zusätzliche Forschungsmittel nach Österreich, etwa aus europäischen Programmen, eingeworben.

Da die meisten befragten Einrichtungen keine explizite Gewinnorientierung aufweisen, oblag die wirtschaftliche Verwertung der Forschungsergebnisse meist den Unternehmenspartnern des Projekts. Hieraus haben sich jedoch zahlreiche strategische Kooperationen zwischen den Forschungseinrichtungen und Unternehmen

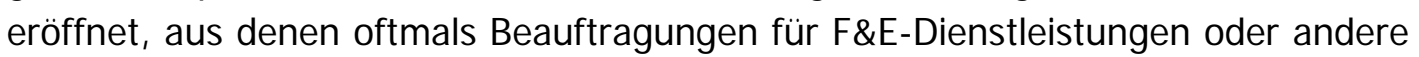
Geschäftsmodelle im Beratungsbereich entstanden sind. Durch den engen Unternehmenskontakt lässt sich jedoch sagen, dass die Haus der Zukunft-Projekte mehrfach zur Entwicklung neuer Produkte und Lösungen geführt haben, welche großteils erfolgreich auf dem Markt platziert werden konnten.

Die wissenschaftliche Verbreitung der Projektergebnisse über Publikationen, Konferenzbeiträge, Vorträge etc. stieß in der Fachcommunity auf große Resonanz. Die innovativen Projekte von Haus der Zukunft in unterschiedlichen Themenfeldern haben den Fördernehmern Forschungsarbeiten in besonderer Qualität ermöglicht, die der von Kollegen aus anderen europäischen Ländern nicht nachsteht. Weiters haben vor allem die Grundlagenforschungsprojekte in Haus der Zukunft den Weg für weiterfolgende Projekte geebnet, die auch in anderen Programmen umgesetzt wurden. Die Gesprächspartner von öffentlichen Universitäten berichteten auch davon, dass die Erkenntnisse aus Haus der Zukunft-Projekten unmittelbar in die Lehre einfließen. Ein weiterer Gesprächspartner hat die Beobachtung angestellt, dass Kollegen von renommierten Universitäten wie MIT oder ETH Zürich ein vielfach höheres Forschungsbudget zur Verfügung haben, aber dennoch auf Konferenzen immer danach fragen, woran in Österreich gerade geforscht wird und welche neuen Erkenntnisse es gibt.

Insbesondere Projekte mit Leuchtturmcharakter haben die nationale und internationale Sichtbarkeit der Forschungseinrichtungen enorm unterstützt. Die Gesprächspartner berichten bspw. von wiederholten Medienanfragen zum Themenfeld Nachhaltiges Bauen, neuen Kooperationen mit Universitäten und privatwirtschaftlichen Partnern oder der Auszeichnung von Projekten mit Preisen. Projekte, deren inhaltliche Basis in Haus der Zukunft gelegt wurde, erhielten zahlreiche internationale Preise, was für die verhältnismäßig kleinen österreichischen Forschungseinrichtungen von enormer Bedeutung ist, um auf internationaler Ebene wahrgenommen zu werden. Die an Haus der Zukunft intensiv beteiligten Universitäten konnten sich auch soweit profilieren, dass nunmehr Doktoratsstudenten aus aller Welt gezielt eine Dissertation im Themenbereich Nachhaltig Bauen in Österreich anstreben und sich um Studienplätze bemühen.

Die generelle Wirkung des Programms Haus der Zukunft wird von den befragten Projektträgern auf mehreren Ebenen gesehen. Zum Einen hat Haus der Zukunft dazu beigetragen, dass die Normen, Standards und Richtlinien im Gebäude- 
bereich deutlich in Richtung Nachhaltigkeit verändert wurden. Dies ist nur möglich, wenn die Entscheidungsträger und Stakeholder von der Sinnhaftigkeit und Machbarkeit von Nachhaltigem Bauen überzeugt sind - die Demonstrations- und Leitprojekte von Haus der Zukunft haben dazu einen großen Beitrag geleistet. Damit geht einher, dass Österreich weltweit die höchste Passivhausdichte aufweist. Weiters konnte mit dem Programm kontinuierlich Forschungsarbeit in Österreich geleistet werden, was nicht nur zum Aufbau hoher Kompetenzen in Wissenschaft und Wirtschaft, sondern auch zur Herausbildung einer Fachcommunity und deren Profilierung auf internationaler Ebene geführt hat. Betrachtet man das langfristige Kosten-Nutzen-Verhältnis, so haben sich die öffentlichen Ausgaben für Haus der Zukunft vielfach rentiert, meinten mehrere Gesprächspartner.

Im Ausblick auf die Zukunft sehen die befragten Projektträger eine Reihe von Forschungs- und Innovationsthemen, die für Österreich relevant wären (siehe Abschnitt „Empfehlungen“). Im Zuge dessen wurde von nahezu allen Gesprächspartnern betont, dass das Programm viel in Österreich aufgebaut und angestoßen hat - ohne Haus der Zukunft wäre Österreich kaum zu einem „Frontrunner“ im Themenfeld Nachhaltiges Bauen geworden. Einige Projektträger äußerten jedoch Sorge, ob diese Position mittel- bis langfristig gehalten werden kann. Die Weiterführung gewisser Themenfelder im Programm "Stadt der Zukunft" wird zwar als logischer Output aus Haus der Zukunft gesehen, kann aber weder thematisch noch finanziell Haus der Zukunft ersetzen. Weiters steht Nachhaltiges Bauen als Thema derzeit nach Einschätzung mancher Experten in der Diskussion, etwa im Zielkonflikt mit der Erfordernis zur Schaffung von günstigem Wohnraum im urbanen Raum. Ferner treten neue Themen auf die Forschungs- und Innovationsagenda (Informations- und Kommunikationstechnologien, Mobilität, Produktionsforschung/ "Industrie 4.0" etc.), was in Verbindung mit immer knapperen öffentlichen Budgets zwangsläufig zu einer nachteiligen Ressourcenallokation führt.

\subsubsection{I nterviews mit relevanten Stakeholdern}

Des Weiteren wurden 11 Stakeholder im Zuge der Evaluierung interviewt. Die Befragten sind in Ministerien, Förderstellen, Verbänden, Landesagenturen und Privatunternehmen tätig und haben das Programm in unterschiedlicher Form, aber durchwegs über mehrere Jahre intensiv begleitet. Eine Liste der Interviewpartner sowie der Gesprächsleitfaden sind im Anhang zum Evaluierungsbericht angeführt.

Die Berühungspunkte mit Haus der Zukunft waren analog zur Heterogenität der befragten Stakeholder sehr unterschiedlich. Nur in Einzelfällen waren Stakeholder an konkreten Projekten beteiligt, die Mehrzahl hatte auf einer strategischen Ebene Kontakt mit dem Programm bzw. den programmverantwortlichen Stellen. Durchwegs wurde die gute und konstruktive Zusammenarbeit aller Beteiligten her- 
orgeheben. Vereinzelt hat sich durch einen beruflichen Wechsel auch die Rolle der Befragten verändert.

Die Programmumsetzung wurde als positiv beurteilt, wobei während der Programmlaufzeit Weiterentwicklungen und Lernprozesse erfolgt sind und in den letzten Ausschreibungen ein sehr hohes Qualitätsniveau erreicht wurde. Einige Gesprächspartner betonten die positive Wirkung der langen Laufzeit des Programms, die den Aufbau fundierter Kompetenzen und Strukturen in Österreich ermöglicht hat. Der "lange Atem der Verantwortlichen“ wird als wesentlicher Erfolgsfaktor gesehen - ebenso die unmittelbare Umsetzung von Forschungsergebnissen im Rahmen von Demonstrations- und Leitprojekten. Mehrfach wurde auch der „Zwang zur Kooperation“ als wichtiges Element betont, da so insbesondere Akteure aus der Bauwirtschaft für Forschung und Innovation aktiviert werden konnten und zugleich eine starke Marktorientierung gegeben war.

Hinsichtlich der ausgewählten Themenschwerpunkte wurde zumeist konstatiert, dass sich das Programm kontinuierlich und logisch weiterentwickelt hat. Die Offenheit der verantwortlichen Akteure für neue Inputs wurde diesbezüglich mehrfach angesprochen. Vereinzelt wurde angemerkt, dass zu Beginn des Programm der inhaltliche Fokus zu eng definiert war - das Themenspektrum des Programms wurde erst im Lauf der Zeit breiter und differenzierter. Mit der hohen Entwicklungsdynamik und dem relativ raschen Schritt vom Passiv- zum Plusenergiehaus könnte allerdings auch - so einige Gesprächspartner - eine gewisse Überforderung der Bauträger und Bauherrn einhergehen, weil der Markt nicht bereit ist, so rasch neue Konzepte aufzunehmen.

Die Begleitmaßnahmen wurden durchwegs als sehr zentrale Bausteine des Programms bewertet. Insbesondere die öffentliche Verfügbarkeit der Projektberichte, themenspezifische Workshops und Transfermaßnahmen haben aus Sicht der befragten Stakeholder zu einem breiten Kompetenzaufbau in Österreich geführt und die Bildung eines "closed club" in der Forschungscommunity verhindert. Nicht zu unterschätzen ist auch die Wirkung des informellen Austauschs im Zuge von Begleitmaßnahmen und die Motivationswirkung, die durch Veranstaltungen, Medienberichte etc. ausgelöst werden kann. So wurde insgesamt eine positive Stimmung für das Thema geschaffen. Eventuell wäre es auch möglich gewesen, so einige Befragten, das Netzwerk noch größer auszuweiten und weitere potenzielle Mitwirkende anzusprechen.

Unisono hoch eingeschätzt wurde die generelle Wirkung des Programms. Haus der Zukunft hat wesentlich dazu beigetragen, das Thema Nachhaltig Bauen in Österreich aufzubauen und die Akteure aus Wissenschaft, Wirtschaft und Verwaltung miteinander zu vernetzen. Haus der Zukunft kann als zentraler Treiber und Impulsgeber des Aufbaus von Forschung und Innovation im Baubereich gesehen werden. Die hohe Innovationsdynamik im Gebäudesektor in Österreich wäre ohne 
Haus der Zukunft kaum vorstellbar. Für die Bewusstseinsbildung waren insbesondere die Demonstrations- und Leitprojekte von großer Wichtigkeit, da sie die Möglichkeiten und Chancen nachhaltiger Bauformen aufzeigten. Von diesen Gebäuden und den vielen Forschungsarbeiten ging eine große Strahlwirkung aus, etwa in Hinblick auf die Entwicklung von Richtlinien, Normen und Standards. Auch in den Bundesländern hat Haus der Zukunft starke Wirkungen entfaltet, etwa in Bezug auf die Wohnbauförderung.

In diesem Zusammenhang wurde auch der Wunsch geäußert, die Ergebnisse von gescheiterten Projekten stärker zu kommunizieren. Die Erkenntnis, dass etwas nicht funktioniert, ist aus fachlicher Sicht ebenso wichtig wie die Erkenntnis, dass ein Ansatz funktioniert. Logisch ist, dass Erfolge gegenüber einem Fördergeber und den Fachkollegen stärker kommuniziert werden als Misserfolge.

Das Programm hat nach Einschätzung der maßgeblichen Stakeholder wesentliche Beiträge zur Erreichung der Zielsetzungen auf verschiedenen Ebenen leisten können:

\section{Bewusstseinsbildung und Sensibilisierung für die Chancen im Bereich Nachhaltiges Bauen}

Haus der Zukunft hat - insbesondere durch die Demonstrations- und Leitprojekte - wesentlich dazu beigetragen, dass sich Nachhaltiges Bauen in Österreich, etwa in Form von Passivhäusern, auf einer breiten Basis etablieren konnte. Auch wurde generell für Forschung und Entwicklung im Bausektor ein wichtiger Impuls gesetzt und es wurden laufend neue Trends und Entwicklungen in den Diskurs eingebracht.

* Auf- und Ausbau der Forschungskompetenzen im Baubereich in Österreich

Die Forschungslandschaft im Bereich Nachhaltiges Bauen hätte sich aus Sicht der befragten Experten vielleicht auch ohne das Progamm entwickelt - mit Sicherheit aber nicht in dieser Geschwindigkeit und Dimension. Der Kompetenzaufbau wurde aber nicht nur finanziell unterstützt, sondern es wurden die Erkenntnisse durch die konsequente Verfolgung des Publizitätsprinzips zugänglich gemacht. Weiters haben die relativ niedrigschwelligen Antragsmodalitäten und der Kooperationsgrundsatz dazu geführt, dass neue Akteure für Forschung und Innovation im Baubereich gewonnen werden konnten.

\section{Vernetzung der relevanten Akteure in Österreich}

Damit hängt unmittelbar zusammen, dass Haus der Zukunft viele für den Baubereich relevante Akteure, etwa aus den Bereichen Wirtschaft, Wissenschaft, öffentliche Institutionen u.dgl., ansprechen konnte. Um nachhaltige Gebäude zu entwickeln und zu realisieren, braucht es viele Kräfte, die an einem Strang 
ziehen. Die Begleitmaßnahmen wurden in diesem Kontext mehrfach erwähnt, da Workshops, Weiterbildungsveranstaltungen uäm. die Netzwerkbildung wesentlich unterstützen. Dennoch wurde angemerkt, dass bestimmte Zielgruppen - genannt wurden etwa Wohnbaugenossenschaften - nur punktuell angesprochen werden konnten.

* Erschließung der Marktchancen österreichischer Unternehmen in Hinblick auf energieeffiziente und nachhaltige Gebäude

Die befragten Stakeholder betonten einerseits die bisherigen Unterstützungsmaßnahmen zur Forcierung der Marktdurchdringung, insb. des Heimmarktes Österreich. Andererseits werden noch große Potenziale in der internationalen Marktdurchdringung gesehen. Einige Unternehmen konnten bereits auf globalen Märkten mit Technologien und Dienstleistungen für Nachhaltiges Bauen reüssieren - in Anbetracht der großen weltweiten Nachfrage werden noch enorme Chancen für österreichische Anbieter in diesem Segment gesehen. Auch wird von manchen Stakeholdern eine gewisse Sättigung des Heimmarktes beobachtet bzw. wird die Markteinführung innovativer Lösungen in Österreich als Herausforderung gesehen, da die Baukosten enorm gestiegen sind und weitere Investitionen in der Bauphase nur bedingt möglich sind.

\section{Stärkung der internationalen Positionierung Österreichs als Kompe- tenzträger im Bereich Nachhaltiges Bauen}

Vor diesem Hintergrund kommt der internationalen Postionierung Österreichs als Kompetenzträger im Bereich Nachhaltiges Bauen eine umso größere Rolle zu. Dies ist durch Haus der Zukunft auf der Forschungsseite sehr stark gelungen, wie die hohe Reputation Österreichs in der Scientific Community und das ungebrochene Interesse ausländischer Delegationen an Haus der ZukunftDemonstrations- und Leitprojekten belegen. Österreich spielt hier in der "Champions League“ - wie ein Gesprächspartner meinte. In wirtschaftlicher Hinsicht werden hier noch Potenziale gesehen (s.o.), wobei zu berücksichtigen ist, dass die österreichischen Anbieter vielfach hochspezialisiert und kleinteilig strukturiert sind. Eine internationale Markterschließung ohne zielgerichtete und umfangreiche Unterstützungsmaßnahmen wird voraussichtlich auf Einzelprojekte begrenzt bleiben.

Die Stakeholder konnten eine starke I mpulswirkung des Programms auf andere Politikbereiche und Maßnahmen auf Bundes- und Länderebene in Österreich beobachten. In erster Linie wurden dabei Normen und Standards erwähnt, die inhaltlich auf die Erkenntnisse von Haus der Zukunft basieren (TQB, klima:aktiv Gebäudestandard etc.), und die Wohnbauförderung der Länder, welche sich zusehends in Richtung Nachhaltigkeit entwickelt hat. Die Forschungsprojekte und Pilotmaßnahmen im Bereich Nachhaltiges Bauen haben allerdings auch einige neue Fragen aufgeworfen, die bislang nicht gelöst werden konnten. Beispielhaft erwähnt sei hier die Problematik der verpflichtenden Überschusseinspeisung von lokal pro- 
duziertem Strom, welcher vor Ort effektiver genützt werden könnte. Wünschenswert wäre aus Sicht der Stakeholder eine stärkere Verbindung von Bau- und Energiepolitik, da Gebäude nicht nur als Energieverbraucher, sondern in Zukunft auch als Energieerzeuger und Energiespeicher fungieren könnten. Dazu sind jedoch ganzheitliche Konzepte auf lokaler Ebene und entsprechende politisch-legistische Weichenstellungen auf Landes-, Bundes- und EU-Ebene erforderlich. Eine Wirkung auf die Forschungspolitik wird von manchen Experten auch gesehen, da manche Elemente von Haus der Zukunft nun auch in anderen Förderprogrammen angewendet werden (Begleitmaßnahmen, verpflichtende Publikation, Leuchtturmprojekte etc.). Sinnvoll wäre es, die Langfristigkeit von Haus der Zukunft - die ein wesentlicher Erfolgsfaktor des Programms war - generell in der Forschungs- und Innovationspolitik zu etablieren. Ausgewiesene Stärke- und Potenzialfelder sollten durch langfristige Forschungsförderung unterstützt werden, anstatt "alle paar Jahre auf ein neues Hype-Thema aufzuspringen."

\subsubsection{Interviews mit internationalen Experten}

Ferner wurden fünf internationale Experten im Rahmen von telefonischen Interviews um ihren Input zum Programm Haus der Zukunft gebeten. Eine Liste der Gesprächspartner findet sich im Anhang. Die fünf befragten Personen sind allesamt Experten im Themenfeld Nachhaltiges Bauen und in unterschiedlichen Funktionen tätig (zB Forschungseinrichtungen oder öffentlichen Institutionen).

Die Berührungspunkte zum Programm Haus der Zukunft bestanden vorrangig in einer Mitwirkung in der Bewertungsjury, die die Projektauswahl vorgenommen. Das Prinzip, diese Jury durch ausländische Experten zu besetzen, wurde durchwegs begrüßt, da es eine objektive Bewertung der Projektanträge sicherstellte. Ferner wurden die organisatorische Vorbereitung und Abwicklung der Jurysitzungen und das fachliche Niveau der Diskussionen in den Jurysitzungen explizit hervorgehoben. Die meisten Befragten haben vermehrt in den letzten Jahren intensiveren Kontakt mit Haus der Zukunft gehabt - in den Anfangsjahren wurde es meist aus der Ferne mit Interesse wahrgenommen.

Augenscheinlich war für die internationalen Experten die Qualität der Projekte, die aus Haus der Zukunft vorgegangen sind. Insbesondere die Demonstrationsund Leitprojekte haben bei den internationalen Experten Eindruck hinterlassen. Eine Gesprächspartnerin, die an einer deutschen Hochschule lehrt, macht mit Studierenden nach wie vor Exkursionen zu Haus der Zukunft-Projekten, da an diesem Gebäuden innovative nachhaltige Bautechnologien besichtigt werden können. Eine Gesprächspartnerin sieht als Teil des „Erfolgsrezepts“ von Haus der Zukunft auch den Umstand, dass die realisierten Gebäude nicht nur technologisch innovativ waren, sondern auch auf ansprechendes Design Wert gelegt wurde. 
Die Wahrnehmung von Haus der Zukunft in der internationalen Fachcommunity und damit auch im Ausland wurde von allen Gesprächspartnern bestätigt. Haus der Zukunft hat - nicht zuletzt durch die umfangreichen Publikationstätigkeiten - eine hohe Sichtbarkeit und Präsenz erlangt. Somit wurde die fachliche Kompetenz der Projektträger, aber auch die strategische Konzeption des Programms über die Grenzen Österreichs hinaus wahrgenommen. "Österreich hat einen guten Ruf in der Branche und steht auf einer Stufe mit Deutschland und der Schweiz" wurde dazu gesagt.

In diesem Kontext steht auch, dass Haus der Zukunft mehrfach in europäischen Gremien vorgestellt wurde und wesentliche Inputs für die Formulierung der EUGebäuderichtlinie gegeben hat. Auch im Rahmen von Veranstaltungen im Ausland wird immer wieder auf Haus der Zukunft bzw. die dabei ermöglichten Projekte verwiesen. Beispielsweise wurde der LifeCycle Tower ONE in Dornbirn - ein Haus der Zukunft-Demonstrationsprojekt - unlängst auf einer Tagung des baden-württembergischen Umweltministeriums vorgestellt.

Die Experten berichten von ähnlichen Programmen, die in anderen europäischen Ländern in den letzten Jahren entstanden sind. Eine direkte Vergleichbarkeit zwischen den Programmen ist aufgrund unterschiedlicher Strukturen, Budgets und politischer Zielsetzungen nicht gegeben. Ein Gesprächspartner berichtet davon, dass bei einer neuen Forschungsfrage zunächst die Haus der ZukunftOnlineplattform konsultiert wird, „da man in Österreich wahrscheinlich schon dazu etwas gemacht hat". Länder wie Großbritannien und die USA forschen schon länger im Bereich Nachhaltiges Bauen, haben aber weniger Ergebnisse vorzuweisen als bspw. Österreich und Deutschland.

Österreich hat sich nicht zuletzt durch Haus der Zukunft international stark positionieren können. In Fachkreisen sind Österreicher sehr präsent. Als wichtiger Faktor wird dabei gesehen, dass die Proponenten des Programms wie das BMVIT selbst in internationalen Kooperationen aktiv sind (zB IEA, ERA-NET), wovon eine große Signalwirkung an die Community ausgeht. Auch auf bilateraler Ebene besteht ein enger Austausch, insb. zu den Pendants in Deutschland und der Schweiz (DACH-Kooperation).

Vereinzelt wurde auch Kritik am Programm geübt. Ein Gesprächspartner sieht am Übergang von der ersten zur zweiten Programmphase eine zu große Ausweitung der Thematik, sodass die eigentliche Zielsetzung des Programms in den Hintergrund gerückt ist und eine Vielzahl an Themenfeldern adressiert wurde. In Hinblick auf die Mehrkosten innovativer Lösungen ist, so ein weiterer kritischer Aspekt, mehr Transparenz und Bewusstsein in der breiten Öffentlichkeit zu schaffen. Minimale Mehrkosten, die eine hohe Hebelwirkung in Hinblick auf die Energieeffizienz bewirken, dürfen nicht unter dem Schlagwort "leistbares Wohnen“ abgelehnt werden. 
Für die Zukunft sehen die internationalen Experten einen großen Trend in der gebäudeübergreifenden Betrachtung und Entwicklung von Stadtteilen. Die IT nimmt in Gebäuden von morgen eine immer stärkere Rolle ein - hierfür sind passfähige Lösungen, die keine Abhängigkeiten von IT-Anbietern schaffen, zu entwickeln und Fragen der Datenerhebung, Datennutzung und Datenauswertung zu klären. Der Nutzer wird darüber hinaus auch in Gebäuden der Zukunft eine zentrale Rolle spielen - das Nutzerverhalten und die sozialen Dimensionen von Bauen und Wohnen sind daher weiterhin zu untersuchen. Weitere Themen sind der Einsatz ökologischer Baustoffe zur Reduktion der "grauen Energie“, die für die Herstellung von Baustoffen und Baukomponenten aufgebracht wird, und bauökologische Fragestellungen (Schadstoffe, Umweltbelastungen, "Wohlfühl-Faktor"), da das Thema Gesundheit in allen Ausprägungsformen in den kommenden Jahren an Relevanz zunehmen wird.

\subsection{Berechnung der volkswirtschaftlichen Effekte}

Der international anerkannte Ökonom Univ. - Prof. Dr. DDr. h.c. Friedrich Schneider und Mag. Jasmin Voigt (Johannes Kepler Universität, Institut für Volkswirtschaftslehre) haben im Zuge der Evaluierung eine volkswirtschaftliche Analyse der regionalen Wertschöpfungs- und Beschäftigungseffekte durch das Programm Haus der Zukunft durchgeführt. Mit Hilfe eines ökonometrisch geschätzten Simulationsmodells können die Wertschöpfungs- und Beschäftigungseffekte, die das Programm stimuliert hat, erfasst werden.

In die Simulationen des Regionalmodells gehen als Primäreffekte die genehmigten Gesamtkosten ein. Hierbei werden im ersten Schritt eine Aufschlüsselung nach den Programmphasen „Haus der Zukunft“ und "Haus der Zukunft Plus" gemacht und in weiterer Folge die Gesamtkosten bzw. -förderungen des Programms betrachtet. Weiters wurden die Projektbeteiligungen nach Organisationstyp (Unternehmen, außeruniversitäre Forschungseinrichtung etc.) in der Berechnung berücksichtigt. Als Basis für die Berechnung gelten die genehmigten Gesamtkosten der Projekte, die sich pro Jahr im Schnitt auf $€ 11,1$ Mio. belaufen.

Bei dem für die Simulationen verwendeten Modell handelt es sich um ein ökonometrisch geschätztes, mittel- bis langfristig orientiertes und sektoral gegliedertes Simulationsmodell, das 16 Wirtschaftsbereiche enthält. In einem interaktiven System von 64 Verhaltens- und 142 Definitionsgleichungen wird das aus der Theorie abgeleitete und anhand tatsächlicher Gegebenheiten beobachtete Wirtschaftsverhalten der Akteure so authentisch wie möglich abgebildet, wobei auch noch 26 exogene Erklärungsfaktoren verwendet werden. 
Das Simulationsmodell kann zweifach unterteilt werden: Sowohl horizontal in fünf Hauptblöcke, in denen die zentralen volkswirtschaftlichen Kenngrößen abgebildet werden, als auch in 16 verschiedene Wirtschaftsbereiche:

\section{Sachgüterproduktionsbereiche:}

$>$ Nahrungs- und Genussmittel

$>$ Textilien und Bekleidung

$>$ Holzbe- und -verarbeitung

$>$ Papiererzeugung und -verarbeitung

$>$ Chemie und Erdölindustrie

$>$ Erzeugung von Stein- und Glaswaren (oder Bauzulieferer)

$>$ Grundmetalle und Metallverarbeitung

\section{Produktionsnahe Dienstleistungsbereiche:}

$>$ Energie- und Wasserversorgung

$\rightarrow$ Bauwesen

$>$ Verkehrs- und Nachrichtenwesen

$>$ Vermögensverwaltung und Wirtschaftsdienste

3. Klassische Dienstleistungsbereiche:

$>$ Handel

$>$ Gastgewerbe und Beherbergung

$>$ Sonstige Dienste

$>$ Öffentliche Dienste

Weiters ist das Simulationsmodell in fünf simultan verbundene Blöcke unterteilt:

* Produktionsblock: Im Produktionsblock werden die Nettoproduktionswerte nach den einzelnen Wirtschaftsbereichen bestimmt.

* Beschäftigungsblock: Im Beschäftigungsblock wird die Zahl der sektoralen Beschäftigten und die Gesamtbeschäftigung als deren Summe bestimmt.

* Investitionsblock: Im Investitionsblock werden die Kapitalstöcke, die Bruttoanlageinvestitionen der Sachgüterproduktionsbereiche bestimmt.

* Einkommensblock: Im Einkommensblock werden die sektoralen Nominallöhne, die Bruttolohn- und -gehaltssumme, die Bruttotransfers, die Abzüge, die Arbeitslosenunterstützung und damit das Masseneinkommen bestimmt.

* Demographieblock: Im Demographieblock werden die Nettomigration, die Erwerbsquote und das Arbeitskräftepotenzial berechnet. 
Die Zusammenhänge zwischen den einzelnen Blöcken sind in nachstehender Figur „Flussdiagramm des Simulationsmodells" aufgezeigt.

Abbildung 44: Flussdiagramm des Simulationsmodells

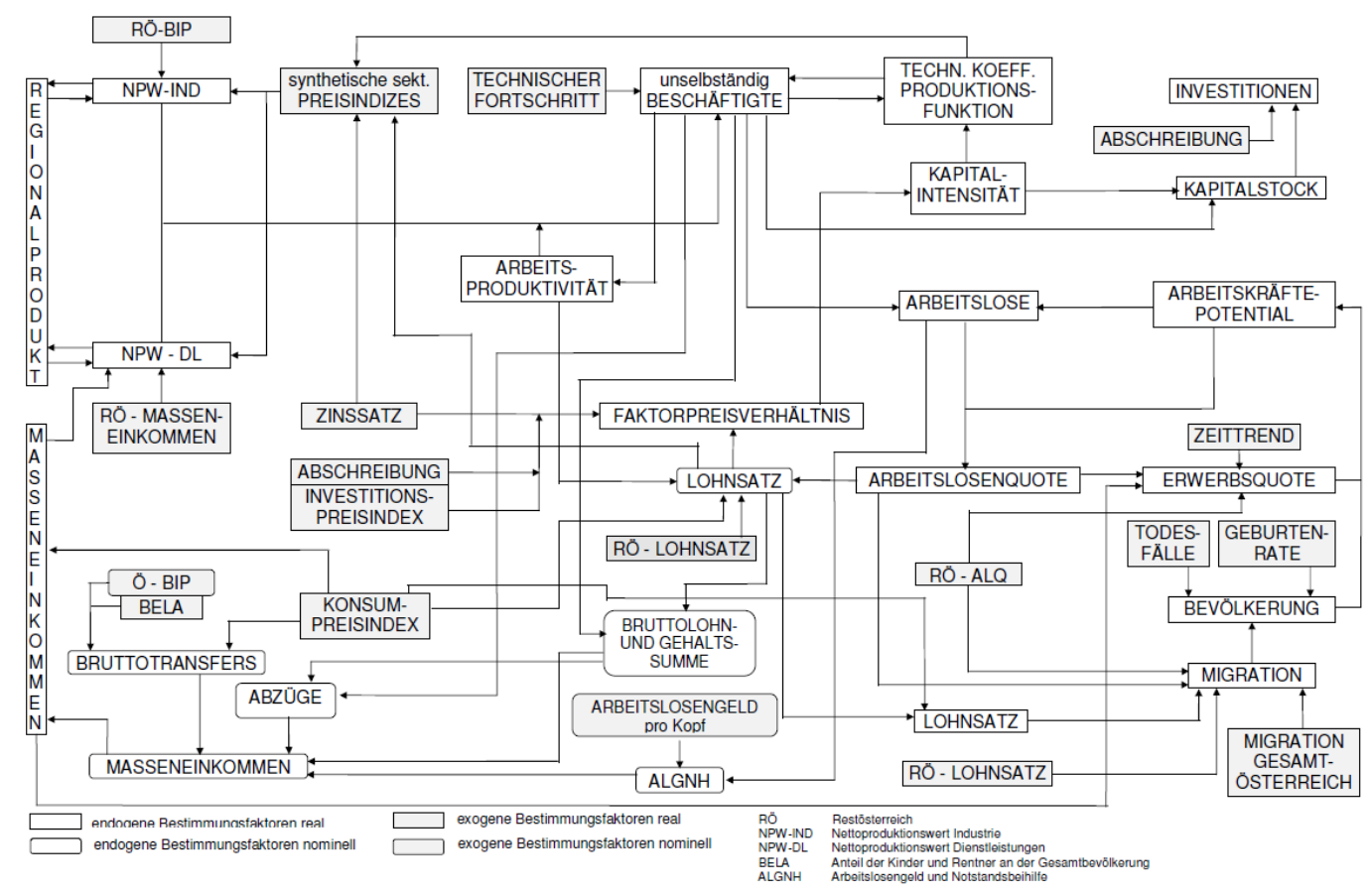

Quelle: Schneider/Voigt (2016)

Mit Hilfe eines derartigen ökonometrisch geschätzten Simulationsmodells ist es möglich, die verschiedensten wirtschaftspolitischen Simulationen durchzuführen. Beispielsweise kann untersucht werden, welchen Effekt eine zusätzliche staatliche Aktivität im Straßenbau oder - wie in diesem Fall - ein Forschungsförderungsprogramm hat. Hierzu werden diese zusätzlichen Summen in das Modell eingeschleust und es wird dann berechnet, welche Primär- und Sekundäreffekte dies für die jeweilige Region auslöst. Mit diesem Modell können Simulationen bis zu 12 Perioden (Jahre) durchgeführt werden. Ökonometrisch geschätzt heißt in diesem Zusammenhang, dass die geschätzten Koeffizienten der einzelnen Verhaltensgleichungen ( $z B$ die Bestimmung der Löhne) aufgrund tatsächlich vorliegender Daten für die einzelnen Bereiche in diesen Bundesländern ökonometrisch ermittelt werden.

Aus diesem Grund ist das ökonometrisch geschätzte Simulationsmodell für eine Vielzahl von Fragestellungen einsetzbar und es ist mit ihm immer möglich, zwei Szenarien zu berechnen: Einmal mit und einmal ohne staatlichen und / oder exogenen Eingriff. Aus der Differenz kann dann zusätzliche Wirkung des staatlichen Eingriffs oder des exogenen Schocks berechnet werden. 
Die Simulationsergebnisse von „Haus der Zukunft“ zeigen, dass die ökonomischen Auswirkungen eindeutig positiv und beträchtlich sind. (Es sei hier ausdrücklich darauf hingewiesen, dass mit Hilfe des ökonometrisch geschätzten Regionalmodells der gesamte volkswirtschaftliche Wertschöpfungseffekt (Ein- und Zweitrundeneffekte) aufgrund des Bestandes der Förderung untersucht wurde und somit eine umfassende volkswirtschaftliche Beurteilung erlaubt):

* In der ersten Programmphase „Haus der Zukunft“ wurde durch die geförderten Projekte im Vergleich zum Basisszenario (kein Förderprogramm) ein zusätzliches Bruttoinlandsprodukt in Höhe von $€ 61,361$ Mio. indiziert. Das zusätzliche Masseneinkommen betrug $€ 26,514$ Mio. und es konnten 494 Beschäftigungsplätze geschaffen bzw. erhalten werden.

* Betrachtet man die zweite Programmphase "Haus der Zukunft Plus“, dann betrugen die Effekte auf das österreichische BIP in Summe $€ 142,841$ Mio. Das zusätzliche Masseneinkommen kann mit $€ 61,722$ Mio. beziffert werden und auch die Anzahl an zusätzlichen Beschäftigten bzw. erhaltenen Arbeitsplätzen ist mit 1.149 beachtlich.

* Das Förderprogamm lief über den Zeitraum von 1999 bis 2013. Berechnet man damit die jährlichen Effekte, dann lassen sich diese für das BIP mit $€ 14,586$ Mio. beziffern. Der durchschnittliche jährliche Effekt auf das Masseneinkommen betrug $€ 6,303$ Mio. und auch die Anzahl von durchschnittlich 117 Beschäftigungsplätzen jährlich ist quantitativ beachtlich und sollte nicht übersehen werden.

* In Summe wurden über die gesamte Programmlaufzeit hinweg also $€$ 204,202 Mio. an zusätzlichem Bruttoinlandsprodukt, ein zusätzliches Masseneinkommen von $€ \mathbf{8 8 , 2 3 6}$ Mio. und 1.643 Arbeitsplätze ausgelöst.

Als Schlussfolgerung kann man aus diesen Ergebnissen der Simulation eindeutig erkennen, dass die volkswirtschaftlichen Effekte durch die Errichtung und Durchführung des Programms Haus der Zukunft quantitativ bedeutend sind. Sicherlich sind sie sowohl für die Wirtschaft als auch für das Land Österreich ein nicht zu vernachlässigender Faktor und stellen im volkswirtschaftlichen Sinn eine bedeutende Größe dar. Es sei auch erwähnt, dass auf diese Weise langfristig und dauerhafte Arbeitsplätze geschaffen werden und darüber hinaus das Land Österreich als Industrie- und Forschungsland zusätzlich an Attraktivität gewonnen hat und auch weiterhin gewinnen wird. 


\subsection{Wirkungsanalyse}

Die Wirkungsanalye fasst die zentralen Wirkungen des Programms Haus der Zukunft zusammen, wobei insbesondere auf die Ergebnisse der Onlinebefragung und der Experteninterviews Bezug genommen wird.

Im Rahmen der Onlinebefragung wurden die Projektträger (Unternehmen, Forschungseinrichtungen und sonstige Einrichtungen) nach ihrer Einschätzung der Wirkungen von Haus der Zukunft befragt. Eine kumulierte Auswertung zeigt, dass die Befragten eine sehr hohe Wirkung in Bezug auf die internationale Profilierung Österreichs als Kompetenzträger im Bereich Nachhaltiges Bauen, einen Beitrag zur Senkung des Energieverbrauchs im Gebäudebereich sowie der Bewusstseinsbildung und Sensibilisierung für Forschung und Innovation im Baubereich in Österreich sehen.

Beachtenswert ist hierbei, dass Unternehmen auch die Bildung von Kontaktnetzwerken zwischen Unternehmen und Forschungseinrichtungen im Baubereich in Österreich als Wirkung von Haus der Zukunft sehr hoch einschätzen. Forschungseinrichtungen wieder konstatieren Haus der Zukunft eine sehr hohe Wirkung in Bezug auf die Bewusstseinsbildung und Sensibilisierung für Forschung und Innovation in der österreichischen Bauwirtschaft. Es wird somit - neben generellen Wirkungen - wesentlich auf Haus der Zukunft zurückgeführt, dass die Vernetzung und der Transfer zwischen Wissenschaft und Wirtschaft unterstützt wurde.

Hohe Wirkungen werden dem Programm hinsichtlich der Sichtbarmachung der Potenziale Nachhaltigen Bauens für die breite Öffentlichkeit, der Stärkung der nachhaltigen Wettbewerbskraft der österreichischen Bauwirtschaft und der Kompetenzsteigerung bei Unternehmen des Bausektors zugeschrieben. Eher geringe Wirkungen konnte Haus der Zukunft bei der Verkürzung der Zeitspanne von der Einzel- zur Serienfertigung innovativer Technologien entfalten. 
Abbildung 45: Was konnte nach I hrer Wahrnehmung durch das Programm „Haus der Zukunft" insgesamt in Österreich bewirkt werden? Bitte geben Sie auf der Skala an, wie hoch Sie die Wirkung einschätzen!

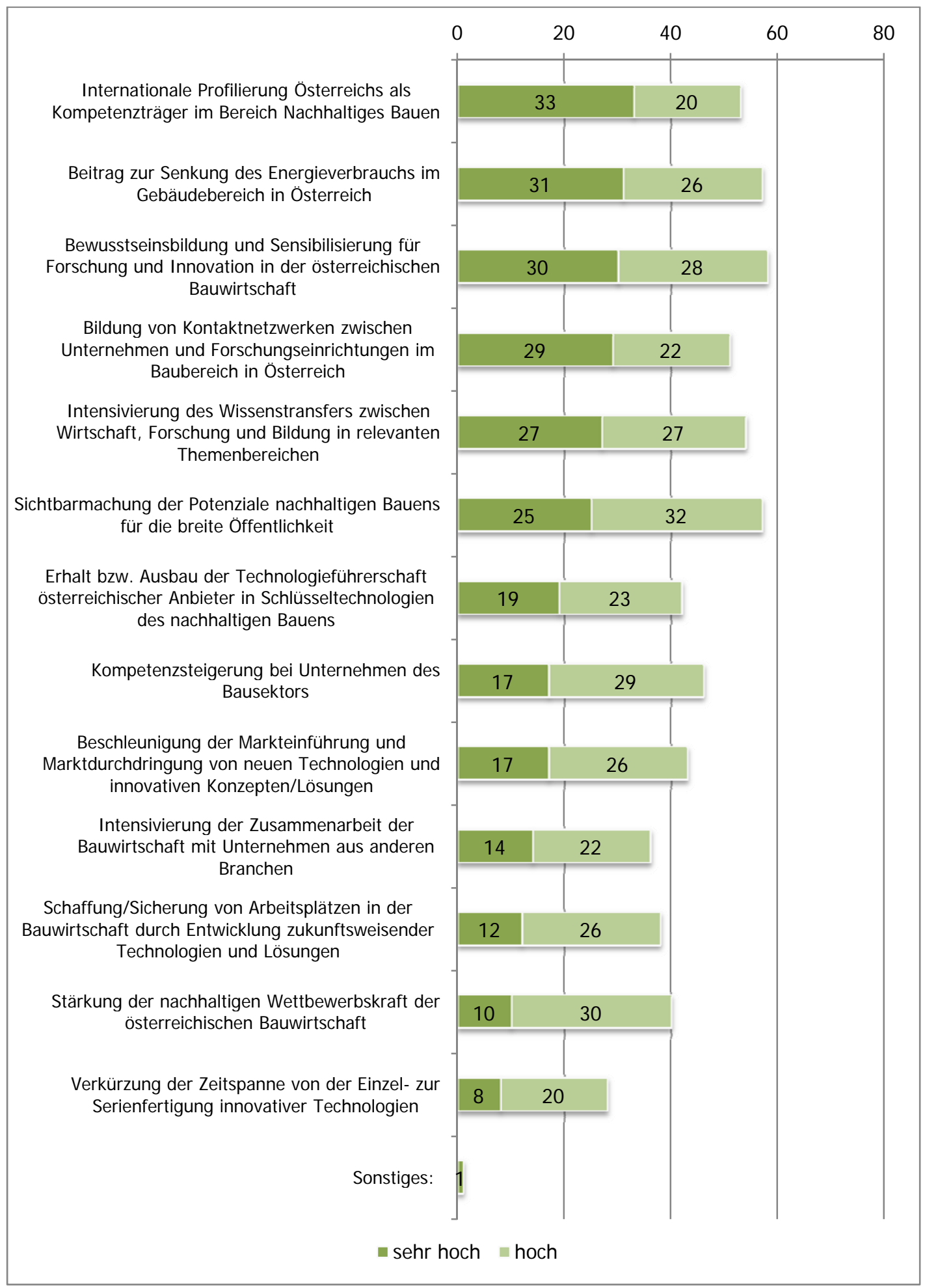

Quelle: Onlinebefragung von Unternehmen, Forschungseinrichtungen und sonstige Einrichtungen; $n=58$ 


\subsubsection{Bewusstseinsbildung und Sensibilisierung für die Bedeutung und Potenziale Nachhaltigen Bauens in Österreich}

Haus der Zukunft war nach einhelliger Einschätzung der Experten ein wichtiger Anstoß für die Bewusstseinsbildung und Sensibilisierung für die Bedeutung und Potenziale Nachhaltigen Bauens in Österreich. Durch das Programm wurde Stakeholdern und der breiten Öffentlichkeit aufgezeigt, welchen Stellenwert das Gebäude für die Erreichung von Klimazielen hat, welche Einsparungsmöglichkeiten realisierbar sind und welcher Mehrwert von nachhaltigen Gebäuden für die Bewohner und Benutzer einhergeht. Passivhäuser und Plusenergiehäuser sind in in verschiedensten Ausführungen vom Einfamilienhaus bis zum Bürogebäude umsetzbar und wirkungsvoll - das wurde durch die Demonstrationsund Leitprojekte deutlich gemacht. Auch wurden die Marktchancen für österreichische Anbieter in diesem Segment aufgezeigt.

Dieses Bewusstsein ist erforderlich, damit Nachhaltiges Bauen in der Breite Anwendung findet. Ein wesentlicher Multiplikator dafür sind Normen, Standards und Förderungen (s.u.). Fachexperten und politische Entscheidungsträger setzen in diesen Regelwerken erst dann Nachhaltigkeitskriterien um, wenn deren praktische Anwendbarkeit unter Beweis gestellt wurde. Hierbei haben die Forschungsarbeiten und Demo-Gebäude von Haus der Zukunft wesentliche Grundlagen geschaffen.

Letzten Endes obliegt es dem Bauherrn, unter Einhaltung der gesetzlichen Vorschriften, nach welchen Maßstäben ein Gebäude errichtet wird. Bauherren sind eine höchst heterogene Gruppe (Privatpersonen, Bauträger, Unternehmen, öffentliche Einrichtungen etc.), sodass die programmbegleitende Öffentlichkeitsarbeit und intensive Verbreitung der Ergebnisse nicht zu geringschätzen sind.

Bei allen realisierten Objekten wurde ein fundiertes und langfristiges Monitoring durchgefürt, sodass die Effekte innovativer Technologien und Baukonzepte nachgewiesen werden konnten. Die Monitoringergebnisse zeigen eine prinzipiell hohe Wirkung in Bezug auf die Energieeffizienz und Zufriedenheit der Benutzer. In Teilbereichen wurden so auch Verbesserungspotenziale aufgezeigt (zB Raumüberhitzung bei großen Glasflächen ohne automatische Beschattung).

Auch die Kostendimension wurde bei den realisierten Objekten berücksichtigt. Grundsätzlich förderte Haus der Zukunft lediglich die Mehrkosten, die durch den Einsatz innovativer Technologien und Lösungen entstanden sind, sodass eine hohe Transparenz über die zusätzlichen Investitionskosten gegeben war. Diese Investitionskosten sind den Einsparungen im laufenden Betrieb gegenüberzustellen, sodass sich die Amortisationszeit und die Lebenszykluskosten berechnen lassen. Ei- 
nige Projekte - exemplarisch genannt sei das Leitprojekt BIGMODERN - haben aufgezeigt, dass mit relativ geringen Mehrkosten in der Größenordnung von $10 \%$ erhebliche Einsparungseffekte erzielbar sind.

Die Haus der Zukunft-Projekte hatten einen klaren Leuchtturmcharakter, da sie unter besonders günstigen Voraussetzungen und unter Mitwirkung von hochkompetenten Experten geplant und errichtet wurden. Sie haben die Möglichkeiten aufgezeigt, die Nachhaltiges Bauen derzeit bietet. Daraus kann jedoch nicht der Anspruch abgeleitet werden, dass alle Gebäude in Zukunft derartige Eigenschaften aufweisen. Vielmehr gilt es, einzelne Aspekte und Lösungen schrittweise in die breite Anwendung zu bringen.

\subsubsection{Berücksichtigung von Nachhaltigkeitskriterien bei Normen, Stan- dards und Förderungen im Baubereich}

Die Errichtung von Gebäuden ist von zahlreichen Regelwerken bestimmt bzw. beeinflusst (rechtliche Vorschriften, technische Standards und Förderungen). Eine zentrale Wirkung von Haus der Zukunft besteht darin, dass - direkt und indirekt Nachhaltigkeitskriterien in diese Regelwerke Eingang gefunden haben und weiterentwickelt wurden.

Als Forschungsprogramm bestand der unmittelbare Beitrag von Haus der Zukunft insbesondere in der Schaffung technischer Grundlagen:

* Das Passivhaus-Konzept wurde insbesondere in der Anfangsphase in zahlreichen Haus der Zukunft-Projekten in Theorie und Praxis vertieft. Es wird von Experten als eine zentrale Errungenschaft des Programms gesehen, dass die Machbarkeit des Passivhausansatzes demonstriert wurde. Österreich wurde binnen weniger Jahre zu einem internationalen Vorreiter in der Passivhaustechnologie und weist in Relation zur Größe des Landes die weltweit größte Dichte an Passivhäusern auf.

* In weiterer Folge wurde an der langfristigen Vision des Plusenergiehauses bzw. der Plusenergiesiedlung geforscht und in Demonstrations- und Leitprojekten erprobt. Obgleich es in der Praxis noch viele Fragen dazu gibt, so markiert dieser Ansatz doch eine entscheidende Trendwende: Das Haus wird vom Energieverbraucher zum Energieerzeuger.

* Passivhaus- und teilweise auch Plusenergiestandards können, wie Haus der Zukunft gezeigt hat, auch in Sanierungen erreicht werden. 
Diese Grundlagen haben sich sukzessive im österreichischen Baurecht niedergeschlagen. Als verpflichtende Rechtsvorschrift für alle Gebäude ist das Baurecht der zentrale Hebel zur Erreichung von Klimazielen und kann - quasi per Gesetz - eine hohe Marktnachfrage nach entsprechenden Technologien und Lösungen eröffnen. Das Baurecht ist zwar auf Länderebene verortet, doch besteht mit dem Österreichischen Institut für Bautechnik (OIB) eine bundesweite Koordinationsplattform. In den OIB-Richtlinien, an denen sich viele Bundesländer orientieren, finden sich jedoch deutliche Bezüge zu Haus der Zukunft-Projekten und Studien. Die EUGebäuderichtlinie, welche in naher Zukunft sehr hohe energetische Standards für Neubauten vorschreibt, wird zu einer weiteren Verschärfung des Baurechts in Bezug auf Nachhaltigkeitskriterien führen.

Ebenfalls in den Zuständigkeitsbereich der Länder fällt die Wohnbauförderung, welche ein anreizbasiertes Steuerungssystem im Wohnbau darstellt und daher ebenfalls eine große Breitenwirkung aufweist. Auch hier ist der Einfluss von Haus der Zukunft auf die Förderungsbestimmungen klar erkennbar, insb. in Hinblick auf die Etablierung des Passivhauses.

Darüber hinaus sind technische Normen und Standards von realiter großer Bedeutung. Haus der Zukunft hat wesentliche Impulse zur (Weiter-)Entwicklung unterschiedlicher Systeme der Gebäudebewertung gegeben:

Total Quality Building (TQB): Die Entwicklung des Gebäudebewertungstools bzw. des Vorläufermodells „Total Quality“ erfolgte als Haus der Zukunft-Projekt in Zusammenarbeit mit der Österreichischen Gesellschaft für Nachhaltiges Bauen, die TQB nunmehr als "Gütesiegel“ für nachhaltige Gebäude einsetzt.

klima:aktiv Gebäudestandard: Die Entwicklung des Schemas zur Zertifizierung von Neubauten und Sanierungen von Wohn- und Dienstleistungsgebäuden erfolgte auf der Basis des TQB-Modells und geht somit auch zu großen Teilen auf Haus der Zukunft zurück.

* Austrian Green Building Standard: Dieser Baustandard wurde im Rahmen von Haus der Zukunft mit dem Ziel entwickelt, die Qualität österreichischen Bauens in nachhaltiger Form international aufzubereiten und kommunizierbar zu machen. Mit dem Label „Austrian Green Building Standard“ soll österreichischen Kompetenzträgern und Anbietern die Positionierung auf ausländischen Märkten erleichtert werden.

* Kommunalgebäudeausweis: Auf Haus der Zukunft geht ferner der Vorarlberger Kommunalgebäudeausweis zurück, welcher den Kommunen bei nachhaltiger und ökologischer Bauweise bis zu 20 \% höhere Bedarfszuweisungen des Landes Vorarlberg ermöglicht. 


\subsubsection{Aufbau und Stärkung der Forschungslandschaft}

Das Programm Haus der Zukunft hat über einen relativ langen Zeitraum ein bestimmtes Forschungsthema adressiert und kontinuierlich neue Aspekte angesprochen. Diese Langfristigkeit und Fokussierung hat den Aufbau und die Stärkung von Forschungsstrukturen in Österreich im Themenfeld Nachhaltiges Bauen ermöglicht. Die im Rahmen von Haus der Zukunft geförderten Projekte und Aktivitäten haben es universitären und außeruniversitären Forschungseinrichtungen ermöglicht, Kompetenzen aufzubauen und zu vertiefen. Weiters konnte Forschungspersonal über die Projekte kofinanziert werden, sodass sich ein Expertennetzwerk herausgebildet hat, welches nunmehr geschätzt 100-300 Personen in ganz Österreich umfasst. Weiters hat das Programm und die kooperative Umsetzung zu einem inhaltlichen Kompetenzaufbau bei den beteiligten Institutionen (insb. BMVIT, FFG, aws, ÖGUT) geführt.

Den relevanten Forschungseinrichtungen wurden durch Haus der Zukunft wesentliche I mpulse für wissenschaftliche Publikationen ermöglicht, die in der internationalen Fachszene nach wie vor auf großes Interesse stoßen. Diese hohe Sichtbarkeit österreichischer Forschungstreibender war wiederum ein wichtiger Türöffner für die Beteiligung an internationalen und europäischen Projekten. Haus der Zukunft - insbesondere die Demonstrations- und Leitprojekte - gilt in der Fachwelt als „Gütesiegel“ für Bauforschung auf höchstem Niveau.

Aufgrund der intensiven Transfer- und Publikationsmaßnahmen blieb das erworbene Wissen nicht auf eine kleine Gruppe von Kernakteuren begrenzt, sondern wurde der breiten Öffentlichkeit zugänglich. Somit wurde auch vermieden, dass sich ein "closed club" herausbildet - vielmehr wurden neue Akteure angesprochen. Nichtsdestotrotz muss festgestellt werden, dass der Kreis der Akteure, die im Bereich Nachhaltiges Bauen über hohe Kompetenzen verfügen, vor dem Hintergrund der gesamten Bauwirtschaftsszene in Österreich relativ klein geblieben ist und hier noch große Potenziale bestehen.

Durch die Instrumente von Haus der Zukunft wurde explizit darauf abgezielt, Kooperationen zwischen Wissenschaft und Wirtschaft im Bereich Nachhaltiges Bauen anzustoßen und zu fördern. Dies ist weitgehend gelungen, wie etwa die Ergebnisse der Onlinebefragung von Unternehmen und Forschungseinrichtungen im Rahmen der vorliegenden Evaluierung unterstreichen. Haus der Zukunft hat als deutlicher Transmissionsriemen zwischen Forschung und Wirtschaft gewirkt. Manche Akteursgruppen (zB Baustoffhersteller, Bauindustrie, Wohnbaugenossenschaften etc.) konnten punktuell in Forschungs- und Innovationsprojekte involviert werden - hier bestehen aber zweifelsohne noch große Potenziale für die Zukunft. 


\subsubsection{Technologie- und Innovationsführerschaft der österreichischen Wirtschaft}

Österreich hat sich in den vergangenen 15-20 J ahren zu einem „Frontrunner“ im Bereich Nachhaltiges Bauen entwickelt - sowohl in Hinblick auf die wissenschaftlichen Kompetenzen wie auch spezialisierte Unternehmen, die als Hersteller und Dienstleister auf nationalen und internationalen Märkten reüssieren. Ferner weist Österreich eine der höchsten Passivhausdichten weltweit auf.

Durch Vorgaben zu Nachhaltigem Bauen - insb. die EU-Gebäuderichtlinie - steigende Energiekosten und ein zunehmendes Bewusstsein für die Vorteile nachhaltiger Bauformen wird sich dieser Markt mit hoher Wahrscheinlichkeit weiterhin sehr dynamisch entwickeln.

Die Technologie- und Innovationsführerschaft Österreichs geht nicht nur, aber zu einem erheblichen Teil auf Haus der Zukunft zurück, denn in den geförderten Projekten wurde viele Grundlagen aufbereitet und die fachliche Basis für innovative Produkte und Lösungen geschaffen. Weiters haben die Demonstrations- und Leitprojekte zu einer raschen Praxiserprobung innovativer Ansätze geführt, die einen großen Erkenntnisgewinn und Know-how-Vorsprung generiert haben.

Haus der Zukunft hat durch seine starke Betonung von Kooperationen viele Unternehmen angesprochen und das Bewusstsein für die Bedeutung von Forschung und Innovation in Unternehmen der Bauwirtschaft maßgeblich gestärkt. Ein solches Bewusstsein ist die zentrale Basis, um einzelne Akteure für Forschungs- und Innovationsprojekte aktivieren zu können, die wiederum eine hohe Strahlwirkung auf die gesamte Branche haben.

Der Bausektor unterscheidet sich in wesentlichen Merkmalen von anderen Wirtschaftszweigen. Zunächst umfasst er sehr viele heterogene Akteure (Planer, Architekten, Baustoffherstellung, Großhandel, Bauunternehmen, Gewerke etc.), die gemeinsame bzw. eng verbundene Wertschöpfungsketten bilden. Ein weiteres Spezifikum ist die Langlebigkeit der Objekte, die sowohl längere Innovationszyklen als in anderen Branchen, aber auch eine hohe Bedeutung der Qualität und zukunftsorientierten Bauweise mit sich bringen. Ferner sind mache Akteure im Bausegment sehr stark regional verankert, andere wiederum stark international orientiert.

Wie die Auswertung der Programmdaten zeigt, haben viele Unternehmen aus der Baubranche an Haus der Zukunft-Projekten teilgenommen. Auffällig ist dabei, dass in erster Linie wissensbasierte Dienstleistungsbetriebe (Planungs- und Ingenieurbüros, Architekten, Forschungsdienstleister) an Haus der Zukunft teilgenommen haben. Unternehmen der Bauindustrie, Baustoffhersteller u.dgl. konnten vereinzelt für Forschungsprojekte gewonnen werden. In Anbetracht der stark ausgeprägten Bauwirtschaft in Österreich bestehen hier zweifelsohne noch Potenziale für 
kooperative) Forschungs- und Innovationsprojekte. Zudem kann Haus der Zukunft als Vorbild für andere FTI-Programme dienen, in denen wissensbasierte Dienstleister - trotz ihrer Schlüsselrolle für die Entwicklung und Umsetzung innovativer Lösungen - nicht oder nur am Rande angesprochen werden.

Diese Dienstleistungsbetriebe neben eine Schlüsselrolle im Planungs- und Bauprozess ein und daher ist es als besonders positive Wirkung von Haus der Zukunft zu bezeichnen, dass die typischerweise kleinteilig strukturierten, spezialisierten wissensintensiven Unternehmen in so hohem Maß an Forschungs- und Innovationsprojekten beteiligt waren. Wissensintensive Dienstleister fungieren als Transmitter und Multiplikatoren von spezifischem Know-how, weshalb vom Kompetenzaufbau in diesem Sektor eine hohe Strahlkraft auf andere Unternehmen ausgeht. Ferner konnten vieler dieser Betriebe indirekt bei der Erschließung internationaler Märkte unterstützt werden - im Export wissensintensiver Dienstleistungen liegt, gerade im Baubereich, noch großes Wachstumspotenzial für den Wirtschaftsstandort Österreich.

\subsubsection{I nternationale Positionierung Österreichs als Kompetenzträger im Bereich Nachhaltiges Bauen}

Haus der Zukunft hat erhebliche Wirkungen in Hinblick auf die internationale Positionierung Österreichs als Kompetenzträger im Bereich Nachhaltiges Bauen entfaltet. Das Programm an sich wurde vielfach als Best Practice-Beispiel vorgestellt, u.a. im Rahmen einer Enquete im Europäischen Parlament.

Forschungsakteure aus Österreich sind in der internationalen Fachcommunity stark präsent, etwa durch die Vorstellung von Ergebnissen der Haus der ZukunftAktiv auf Konferenzen und Tagungen. Ferner konnten durch Haus der ZukunftProjekte Zugänge zu internationalen Forschungsnetzwerken und EU- bzW. IEAProgramme erlangen und Österreich wurde als Forschungsstandort international sichtbar.

Unternehmen mit einer Spezialisierung auf Nachhaltiges Bauen positionieren sich durch Vertriebsaktivitäten und Referenzprojekte zunehmend auf ausländischen Märkten, was das Image Österreichs als „Frontrunner“ im Thema Nachhaltiges Bauen weiter unterstützt.

Die Demonstrations- und Leitprojekte, die im Rahmen von Haus der Zukunft errichtet werden konnten, erfahren eine hohe internationale Resonanz. Zahlreiche Delegationen aus aller Welt haben die Bauten in Österreich besucht. Eine japanische Delegation besuchte beispielsweise auf einer Europareise drei Projekte in 
London, Stockholm und Graz. Österreich spielt, wie dieses Beispiel unterstreicht, in Hinblick auf Nachhaltiges Bauen eindeutig in der "Champions League“ Europas.

Die internationale Sichtbarkeit und fachliche Qualität der Projekte unterstreichen die Preise und Auszeichnungen für Haus der Zukunft-Projekte oder Bauten, die unmittelbar aus Projekten hervorgangen sind.

* „Alles PALETTE“ entstand als Studentenprojekt an der TU Wien: 800 gebrauchte Holzpaletten werden zu Modulen verbaut, die rasch zu einem Haus zusammengefügt werden können. Der mehrschichtige Aufbau eignet sich für den Einbau von Installationen, Dämmung und Beleuchtung. Ein temporäres Palettenhaus wurde zunächst in Wien-Aspern, anschließend in Südafrika realisiert. 2007 gewann das Palettenhaus den EU-weiten Wettbewerb "GAU:DI“ und wurde bei der Biennale in Venedig 2008 ausgestellt.

* Das LISI-Haus, ein Haus der Zukunft-Demonstrationsprojekt, wurde 2013 in die Endausscheidung des "Solar Decathlon" - ein Preis des US-amerikanischen Energieministeriums für nachhaltige Baukonzepte - aufgenommen. Das Projektteam unter Leitung der TU Wien hat das LISI-Haus in Irvine (Kalifornien) für mehrere Wochen aufgebaut. Unter 19 Projekten hat sich das LISI-Haus durchgesetzt und den "Solar Decathlon“-Preis erhalten.

* Das "Sheikh Zayed Desert Learning Center" in den Vereinigten Arabischen Emiraten wurde unter Leitung eines Wiener Architekturbüros und unter Mitwirkung zahlreicher österreichischer Firmen 2014 fertiggestellt. Dabei wurde ein innovatives Konzept für nachhaltige Gebäude unter extremen klimatischen Bedingungen realisiert. Durch den Einsatz unterschiedlicher Technologien und Lösungen, die vielfach in Haus der Zukunft-Projekten entwickelt wurden, kann das Gebäude trotz extrem hoher Außentemperaturen 80 \% des Kühlenergiebedarfs autark generieren. Das Bauwerk wurde durch das amerikanische Programm LEED (Leadership in Energy and Environmental Design) mit dem LEED Platinum Standard zertifiziert und als erstes Gebäude der Emirate mit dem arabischen Green Building Gütesiegel „ESTIDAMA 5 pearls“ ausgezeichnet

* 2015 wurde das recyclebare und energieautarke "Zero Carbon Resorts"Demonstrationsgebäude auf den Philippinen im UNO-Hauptquartier in New York mit dem "Global Human Settlements Award on Planning and Design" ausgezeichnet. Die Grundlagen für das Projekt wurden in Haus der Zukunft entwickelt und im Demonstrationsprojekt "S-House“ in Böheimkirchen erstmals angewandt. Die Realisierung des Gebäudes auf den Philippinen wurde aus EUMitteln im EuropeAid-Programm gefördert. Die "Gruppe Angepasst Technologie“ der Technischen Universität Wien hat daran maßgeblich mitwirkt.

* Ein Büro- und Wohngebäude in Passivhausqualität wurde im chinesischen Zhuozhuo Anfang 2015 mit dem „Austrian Green Building Star“ ausgezeichnet, den BMVIT und BMLFUW initiiert haben. Das Projekt basierte in wei- 
ten Teilen auf Haus der Zukunft-Projekten und wurde von einem österreichischen Bauphysikbüro konzipiert. Bemerkenswert ist hierbei, dass die chinesischen Bauherrn auf das relativ kleine Planungsbüro in Österreich herangetreten sind: Man hat gezielt analysiert, welche Länder über herausragendes Know-how im Passivhausbau verfügen - Österreich war dabei bald auf der Agenda - und hat innerhalb Österreich die Top-Experten gesucht.

Wie diese Beispiele zeigen, weist Österreich noch große Potenziale im Export von Know-how im Bereich Nachhaltiges Bauen auf. Hierbei ist allerdings zu berücksichtigen, dass die diesbezügliche Forschungs- und Unternehmenslandschaft in Österreich meist sehr spezialisiert und dementsprechend kleinteilig strukturiert ist. Eine aktive Unterstützung der Markterschließung durch die relevanten Institutionen und Programme ist daher erforderlich. 


\section{OUTLOOK: Schlussfolgerungen und Empfehlungen}

\subsection{Schlussfolgerungen}

Die Evaluierung zeigt, dass Haus der Zukunft sehr deutliche und nachhaltige Wirkungen erzielt hat. Das Programm war ein maßgeblicher Stimulus, um die Technologiekompetenzen und die Marktposition österreichischer Akteure im Bereich des Nachhaltigen Bauens frühzeitig zu entwickeln und zu vertiefen. Das breite Spektrum an Wirkungen ist besonders beachtlich, da Haus der Zukunft als Forschungsförderungsprogramm per se einen begrenzten Wirkungskreis hat, aber über diesen unmittelbaren Wirkungsbereich hinaus weitere Entwicklungen anstoßen konnte.

Diese positiven Effekte lassen auf eine Reihe von Erfolgsfaktoren zurückführen:

* Ein zukunftsweisendes Thema - Nachhaltiges Bauen - wurde frühzeitig erkannt und durch ein passfähiges Programm gezielt forciert.

* Das Programm hat sich in Hinblick auf die Themenschwerpunkte und Projektarten analog zum Fortschritt der Vernetzung, des Know-hows und der Marktkapazitäten der Akteure weiterentwickelt. Damit wurden permanent neue Impulse zur Weiterentwicklung gesetzt und eine Reihe neuer sinnvoller Förderinstrumente etabliert, die auf das gesamte österreichische FTIFörderinstrument gewirkt haben.

* Dabei wurde die Fachcommunity einbezogen und deren Anregungen für neue Maßnahmen eingeholt und nach Möglichkeit berücksichtigt.

* Neben der monetären Förderung haben insbesondere die Begleitmaßnahmen dazu beigetragen, eine Fachcommunity in Österreich aufzubauen und zu vernetzen, die Öffentlichkeit für das Thema Nachhaltiges Bauen zu sensibilisieren, Projektergebnisse gezielt zu verbreiten uvam.

* Die Projektergebnisse wurden auf der Online-Plattform öffentlich zugänglich gemacht. Damit wurde die Bildung eines "closed club“ vermieden und die Ergebnisse konnten für neue Projekte und Initiativen herangezogen werden.

* Der Aufbau von Kompetenzen, Netzwerken und Strukturen braucht Zeit: Die Langfristigkeit von Haus der Zukunft als Förderprogramm über fast 15 Jahre war sehr wichtig, um einen schrittweisen und fundierten Auf- und Ausbau der österreichischen Kompetenzen zu ermöglichen.

* Neue Zielgruppen und Akteure wurden sukzessive angesprochen und aktiv zur Programmmitwirkung angesprochen. Es konnten insbesondere Unternehmen wie wissensbasierte Dienstleister zur Beteiligung an Forschungsprojekten 
gewonnen werden - diese Unternehmensgruppe, die selten in FTIProgrammen angesprochen wird, spielt aber eine Schlüsselrolle für die Entwicklung und Verbreitung von Innovationen.

* Die Demonstrationsprojekte und Leitprojekte haben die Möglichkeiten und Vorteile Nachhaltigen Bauens praktisch demonstriert und angreifbar gemacht.

* Nicht zuletzt dadurch wurde eine starke internationale Sichtbarkeit und Positionierung Österreichs als Vorreiter Nachhaltigen Bauens erreicht.

Das Themenfeld Nachhaltiges Bauen hat in der letzten Dekade an Bedeutung weiter zugenommen und weist noch große Forschungs- und Innovationspotenziale auf. Immer striktere Rechtsvorschriften, etwa im Zuge der Umsetzung der EUGebäuderichtlinie, und die weiterhin ambitionierten Klimaziele werden in Österreich und Europa über viele Jahre hinweg einen Markt für innovative Technologien und Lösungen im Gebäudebereich sicherstellen. Ferner wird die globale Nachfrage nach entsprechenden Kompetenzen massiv zunehmen - treibende Faktoren sind hierbei das hohe Bevölkerungswachstum in Schwellenländern, die wachsende globale Mittelschicht, die prognostizierte Wachstumsdynamik in Schwellenländern, die Auswirkungen des Klimawandels uvam.

Vor diesem Hintergrund sind auch die Herausforderungen und offenen Fragen zu nennen, die durch die bisherigen Aktivitäten noch nicht umfassend adressiert werden konnten:

* Internationale Marktüberführung innovativer Technologien "Made in Austria"

* Breitenwirksamer Einsatz energieeffizienter, über die rechtlichen Auflagen hinausgehender Gebäudetechnologien in Neubau und Sanierung in Österreich

* Kostensenkung bestehender Technologien / Entwicklung neuer kostengünstiger Lösungen zur Ermöglichung von leistbarem und nachhaltigem Wohnraum

* Integration des Gebäudes in das gesamte Energiesystem, zB Verbindung von Gebäudeenergie mit Mobilitätsenergie

* Lösung rechtlicher Fragestellungen (zB Überschusseinspeisung, Problematik der ungleichen Verteilung von Investition und Nutzen bei Mietwohnungen) ${ }^{7}$

* Industrielle Fertigung skalierbarer und exportfähiger Technologien

* Sicherstellung einer hohen Bauausführungsqualität

* Akzeptanz und Einbindung der Gebäudenutzer

7 Die Mehrkosten nachhaltiger Bautechnologien trägt in der Regel der Bauherr, den Nutzen in Form von niedrigeren Betriebskosten hat jedoch der Mieter - dadurch besteht für den Bauherrn kein monetärer Anreiz, in innovative Technologien zu investieren. 
Diese Themen liegen nicht unmittelbar im Fokus eines F\&E-Programmes wie Haus der Zukunft, aber sie zeigen doch auf, dass trotz der großen Erfolge östereichischer Forscher und Unternehmen im Bereich Nachhaltigen Bauens in den letzten 15 Jahren das Thema keineswegs an einem Endpunkt angelangt ist. Vielmehr sind nun die Kompetenzen aufgebaut und Netzwerke geschaffen worden, um in einem nächsten Schritt wesentliche Hürden - technologischer, rechtlicher, wirtschaftlicher, sozialer Art - gezielt zu adressieren und Nachhaltiges Bauen im Inund Ausland mit noch größerer Dynamik zur Umsetzung zu bringen.

\subsection{Empfehlungen}

Die Evaluierung zeigt ein herausragend positives Ergebnis. Das Programm war sowohl in Hinblick auf die thematische Ausgestaltung wie auch die Wahl und Weiterentwicklung der Instrumente, die operative Umsetzung und die erzielten Wirkungen beispielgebend für praxisnahe, zukunftsweisende FTI-Programme. Die Begleitmaßnahmen sind hierbei besonders zu erwähnen. Die Zielsetzungen des Programms konnten in beiden Programmphasen erreicht werden.

Daher wird eine Weiterführung der Aktivitäten zur Unterstützung von Forschung und I nnovation im Themenfeld Nachhaltiges Bauen angeregt, um die internationale Forschungs- und Innovationsposition österreichischer Akteure in diesem zukunftsweisenden Feld zu stärken und auszubauen. Diese Fortsetzung kann im Rahmen bestehender Initiativen und Programme sowie durch Vernetzungs- und Kooperationsaktivitäten erfolgen.

Folgende Empfehlungen werden seitens der Evaluatoren ausgesprochen:

\subsubsection{Vertiefung der Forschungsförderung im Bereich Nachhaltiges Bauen}

Haus der Zukunft hat rege Forschungsaktivitäten in Wissenschaft und Wirtschaft im Themenbereich Nachhaltiges Bauen stimuliert. Binnen relativ kurzer Zeit konnte sich Österreich damit auf internationaler Ebene als "Frontrunner" positionieren. Diese Position gilt es weiter auszubauen und diesbezügliche Forschungsaktivitäten effektiv zu unterstützen - denn mittlerweile haben auch andere Länder das große Zukunftspotenzial der Thematik erkannt und setzen massive Anstrengungen, um auf das österreichische Kompetenzniveau anzuschließen. 
Folgende Aktivitäten werden dazu vorgeschlagen:

* Fortsetzung bzw. Intensivierung der Adressierung von Forschungsfragen in Bezug auf Einzelgebäude im Rahmen des Programms "Stadt der Zukunft" und ggf. weiterer Forschungsförderungsprogramme

* Weiterführung der praktischen Umsetzung von Forschungsergebnissen im Rahmen von Demonstrationsprojekten oder Pilotprojekten ähnlicher Art, um neue Konzepte und Technologien sichtbar zu machen, sie zu verbreiten und Awareness - in der Bauwirtschaft und darüber hinaus - für die Chancen nachhaltigen Bauens zu schaffen

* Fortsetzung des innovativen Ansatzes der Haus der Zukunft-Leitprojekte, deren Prozessorientierung durch ein mehrphasiges, prozessorientiertes Bündel von Einzelprojekten unter einem gesamtheitlichen und monetär abgegoltenen Projektmanagement eine besonders hohe Wirksamkeit und Sichtbarkeit hervorgebracht hat. Daher wird empfohlen, diese Erfahrungen und Vorgangsweise auf Nachfolgeprogrammen wie Stadt der Zukunft zu übertragen. Die derzeit im Instrumentenportfolio der FFG vorgesehenen Leitprojekte sind als "strategisch und inhaltlich definierte Forschungs- und Entwicklungsprojekte von substantiellem Umfang hinsichtlich inhaltlichem und finanziellem Projektvolumen, der Anzahl an Projektpartnern und der Laufzeit" definiert und unterscheiden sich daher grundlegend von den Haus der Zukunft-Leitprojekten.

Berücksichtigung der langen Zeitdauer von Bauvorhaben in der Gestaltung entsprechender Fördermaßnahmen, da Bauprojekte - im Gegensatz zu industriellen Forschungsprojekten - eine lange Vorlauf- und Umsetzungsdauer haben. Manche Leitprojekte haben ca. 10 Jahre von der ersten Idee bis hin zum Monitoring der Ergebnisse in Anspruch genommen.

* Prüfung neuer Instrumente und innovativer Ansätze der F\&EFörderung in Hinblick auf deren Anwendbarkeit im Bereich Nachhaltiges Bauen, wie etwa das ab Frühjahr 2016 neu eingesetzte Instrument „Innovationslabor“, dessen Ziel die Unterstützung der Schaffung von realen Entwicklungsumgebungen mit der notwendigen materiellen und immateriellen Infrastruktur ist, um nutzerzentrierte Innovationsvorhaben zu ermöglichen und Forschungsergebnisse in marktfähige Produkte und Dienstleistungen zu übersetzen

* Anerkennung der Bedeutung der vorwettbewerblichen Forschung, insb. der ingenieurwissenschaftlichen Basisforschung, der Forschungsleistungen von wissensbasierten Dienstleistern und der sozialwissenschaflichen Forschung durch gezielte Ansprache in relevanten Förderprogrammen ...

* ... und die Gewährleistung attraktiver Förderkonditionen: Prüfung und wenn möglich Adaptierung der Instrumente und Kostenstrukturen von F\&EProgrammen in Hinblick auf die Passfähigkeit für Klein- und Mittelunternehmen, um wissensbasierten Dienstleistungsunternehmen die Beteiligung an 
F\&E-Projekten zu wirtschaftlich vertretbaren Konditionen zu ermöglichen. Laut aktuellem Kostenleitfaden der FFG ist für an einem Projekt mitarbeitende Gesellschafter und mitarbeitende Eigentümer ein Pauschalstundenstatz von $€ 35$ pro Stunde vorgesehen. Das entspricht einem Tagsatz von $€ 280$. Dies verhindert de facto die Beteiligung einer für das Programm sehr wertvollen Zielgruppe, da F\&E-Projekte zu diesen Konditionen wirtschaftlich nicht vertretbar sind.

* Gebündelte Kommunikation der diesbezüglichen Forschungsförderungsangebote an die relevante Zielgruppe unter Einbindung von Multiplikatoren und digitalen Medien (insb. Website www.hausderzukunft.at und Newsletter über die Programmlaufzeit hinaus)

\subsubsection{Adressierung von richtungsweisenden Forschungsthemen mit ho- hem Zukunftspotenzial}

In den 15 Jahren von Haus der Zukunft wurden viele Grundlagen für nachhaltige Gebäude geschaffen (Passivhaus, Plusenergiehaus, Sanierung etc.). Dabei haben sich jedoch viele weitere Themenfelder offenbart, in denen Ansatzpunkte für die Energie- und Ressourceneinsparung im Gebäudesektor bestehen. Darüber hinaus sind viele technische, rechtliche und sozialwissenschaftliche Fragestellungen noch offen. Ferner besteht eine Reihe von Themenfeldern mit großem Zukunftspotenzial (zB kostengünstige und exportierbare Lösungen für Schwellenländer mit hohem Bevölkerungswachstum, Integration und Nutzung von IT und digitalen Technologien in Gebäuden, Sanierungslösungen, Kühlung uvam.). Daher wird angeregt, insb. folgende Themenfelder im Rahmen von "Stadt der Zukunft“ und anderen Forschungsprogrammen zu adressieren:

* Verfahren und Technologien für die nachhaltige Sanierung (zB vorgefertigte Fassadensysteme, Lösungen zur energieoptimierten Aufstockung und Nachverdichtung, Konzepte für die „bewohnte Sanierung“, Energieoptimierungsmodelle für Gebäude unter Denkmalschutz, Kostentransparenz Sanierung vs. Neubau) ${ }^{8}$

Leistbares nachhaltiges Wohnen: Kostensenkung von bestehenden Technologien und Lösungen zur Energieoptimierung, Modelle zur raschen und realistischen Erfassung von Lebenszykluskosten, Entwicklung von „MidtechLösungen" (optimales Gleichgewicht zwischen Nachhaltigkeit und Leistbarkeit), Anpassung der rechtlichen Rahmenbedingungen wie bspw. der Wohnbauförderung und der Kostengrenzen im sozialen Wohnbau

8

Insbesondere im Ausland besteht ein großer Bedarf an Sanierungslösungen - österreichische Unternehmen haben in diesem großen Markt einen international sehr guten Ruf. Zudem bietet die Sanierung im Inland einen größeren Wirkungseffekt auf den Energieverbrauch in Gebäuden, da mit einer nachhaltigen Sanierung eine reale Reduzierung des Energieverbrauchs im Gebäudesektor erreicht werden kann. Bei Neubauten ist das nur bei Passiv- und Plusenergiehäudern der Fall, die nach wie vor nur einen geringen Anteil unter allen Neubauten einnehmen. 
*Digitales Bauen“: Entwicklung und Verbreitung von Lösungen für die optimale Nutzung moderner IT-Systeme in Hinblick auf die Energieoptimierung (zB Prognoserechnungen zum Strom-, Wärme- und Kältebedarf) sowie in Hinblick auf eine Qualitätssteigerung und Kostenoptimierung im Bauprozess; ggf. Einrichtung einer Plattform unter Einbeziehung aller maßgeblichen Akteure der Bauwirtschaft, wissensbasierte Dienstleister wie Architekten, Planer, Bauphysiker, IT-Experten etc. nach Vorbild der deutschen Plattform „Planen Bauen 4.0“

* Lüftung und Kühlung: Entwicklung und internationale Verbreitung von innovativen Technologien und Lösungen zur Kühlung von Gebäuden (siehe bspw. das aus Haus der Zukunft hervorgegangene "Sheikh Zayed Desert Learning Center", das trotz extrem hoher Außentemperaturen 80 \% des Kühlenergiebedarfs autark und nachhaltig generieren kann)

* Erfassung und Optimierung aller energieverbrauchenden Einheiten im Gebäude: Meist steht die Wärme- und Kühlenergie eines Gebäudes im Mittelpunkt der Betrachtung - viele Einheiten im Gebäude verbrauchen allerdings auch Energie (Beleuchtung, technische Geräte, Haustechnik etc.) - die Energieeinsparungspotenziale all dieser Einheiten gilt es zu erfassen, darzustellen und zu optimieren und ggf. neue energieeffiziente Lösungen zu entwickeln

* Bauteilaktivierung zur lokalen thermischen und energetischen Speicherung: Nutzung des Gebäudes bzw. einzelner Teile als Speicher von Energie, Klärung rechtlicher Fragen und Erforschung der sinnvollen Schnittstellen zur Energieerzeugung und - nutzung in unterschiedlicher Form (zB Speicherung der überschüssigen Sonnen- und Windenergie am Tag im Gebäude, Energieabgabe über Nacht in ein Elektrofahrzeug)

* Entwicklung skalierbarer Technologien und Konzepte: Zusammenfassung von Einzeltechnologien und Lösungen in passfähige Gesamtpakete bzw. kombinierbare Module, um die Marktdurchdringung dieser ganzheitlicher Lösungen vorzubereiten

* Gebäude im Verbund: Weiterführung der Forschungsaktivitäten in Bezug auf die nachhaltige Stadtteilentwicklung (insb. „Stadt der Zukunft“) und Entwicklung von äquivalenten Modellen und Lösungen für den ländlichen Raum („Dorf der Zukunft“)

* Durchführung von sozialwissenschaftlichen Forschungsprojekten, zB zu Wohnbedarfen der Zukunft, Wandlung der Wohn- und Bürobedarfe etc.

* Ökologisches Bauen: Einsatz ökologische Dämmstoffe und Baumaterialien, gesundheitsbezogenes Bauen, Reduzierung von Schadstoffen/Allergenen etc. ${ }^{9}$

9 Gesundheit ist ein Mega-Trend, der für die nahe Zukunft große wirtschaftliche Potenziale verheißt. Die Verbindungen mit Bauen und Wohnen sind vielfältig - so bringt bspw. die Wohnraumlüftung nicht nur energetische Vorteile mit sich, sondern bringt für Pollenallergiker eine große Erleichterung. 


\subsubsection{Umsetzung von Transfer- und Vernetzungsmaßnahmen im Rah- men geeigneter I nstrumente}

Die hohe Wirksamkeit von Haus der Zukunft ist zu einem nicht unwesentlichen Teil auf die Begleitmaßnahmen zurückzuführen. Diese waren in Hinblick auf das Fördervolumen zwar nur ein geringes Element des Programms, haben aber essentiell dazu beigetragen, eine Fachcommunity in Österreich aufzubauen und international sichtbar zu machen. Die Praxis zeigt, dass Vernetzung und Kooperation in der Regel einen externen Anstoß und einen Rahmen brauchten, um über längere Zeit aufrecht zu bleiben. Daher wird empfohlen, im Rahmen geeigneter Instrumente Transfer- und Vernetzungsmaßnahmen zu setzen, die - ebenso wie bei Haus der Zukunft - durch eine 100 \%-ige Finanzierung und Laufzeit von bis zu zwei Jahren eine hohe Initialwirkung entfalten können.

Folgende Ansatzpunkte werden dazu festgehalten:

* Weiterentwicklung der Online-Plattform www.hausderzukunft.at: Kontinuierliche Pflege und Aktualisierung der Website, zeitgemäße und nutzergerechte Aufbereitung der Inhalte (zB Verbesserung der Suchfunktion über die 1.320 Publikationen der Website)

* Zentrale Veröffentlichung der Projektberichte von gebäuderelevanten F\&E-Projekten aus unterschiedlichen Förderprogrammen auf der Website und diesbezügliche Verankerung in den Programmrichtlinien bzw. Förderverträgen

* Aktive Unterstützung des Wissenstransfers: Einbringung von Wissen über Nachhaltiges Bauen insb. in die berufliche Bildung (Berufsschulen, Bauakademien etc.) und Qualifizierungsinitiativen

* Programmübergreifende Transfermaßnahmen wie $z B$ themenspezifische Workshops zur Vernetzung gebäuderelevanter Projekte und Akteure aus unterschiedlichen Förderprogrammen

* Unterstützung der Einbringung der Ergebnisse von Haus der Zukunft in baurechtliche Normen, Standards und Förderungsgrundlagen als „Umsetzungsturbo" für nachhaltige Gebäudetechnologien

* „Kompetenznetzwerk Nachhaltiges Bauen“: Vernetzung, Unterstützung und Weiterentwicklung der österreichischen Kompetenzträger im Bereich Nachhaltiges Bauen, zB durch Sichtbarmachung der Kompetenzen nach Vorbild des "Kompetenzkatalog Smart Cities" und des gemeinsamen internationalen Marktauftritts

* Aktive Unterstützung und Forcierung des internationalen Technologietransfers: Präsenz und Sichtbarkeit österreichischer Akteure in internationalen Gremien und Forschungsplattformen, Bekanntmachung österreichischer Initiativen wie zB „Austrian Green Building Star" 


\subsubsection{I mpulse von „Haus der Zukunft“ für den Forschungs- und I nnova- tionsstandort Österreich}

Haus der Zukunft hat nicht nur neue technologische und wissenschaftliche Erkenntnisse hervorgebracht, sondern weit darüber hinausreichende Impulse für Nachhaltiges Bauen in Österreich gesetzt. Diese I mpulskraft gilt es nun gezielt zu nutzen und dieses zukunftsweisende und chancenreiche Themenfeld weiter zu forcieren. Weiters können "lessons learned" aus dem Programm Haus der Zukunft für die österreichische Forschungs- und Innovationspolitik gezielt abgeleitet und berücksichtigt werden. Folgende Möglichkeiten werden dazu gesehen:

* Entwicklung einer breit angelegten und von der Wirtschaft mitgetragenen „Österreichischen Bauinnovationsstrategie" unter breiter Beteiligung der maßgeblichen Akteure als gemeinsame Roadmap für die Verwertung vorhandenen Wissens, die internationale Markterschließung und Vernetzung

* Aktive Maßnahmen zur gezielten Positionierung Österreichs als Kompetenzregion für Nachhaltiges Bauen in enger Kooperation mit den relevanten Einrichtungen (insb. WKO/Aussenwirtschaft Austria, Austrian Business Agency), evt. auch in Kombination mit der hohen Lebensqualität und dem bauhistorischen Erbe Österreichs im Außenmarketing Österreichs

* Nutzung der Potenziale des öffentlichen Sektors als Vorreiter und Innovationstreiber im Bereich Nachhaltiges Bauen durch Vergabe öffentlicher Bauaufträge nach den Lebenszykluskosten anstelle der reinen Errichtungskosten („Bestbieterprinzip“ im Sinne der gesamten Lebensdauer des Gebäudes) sowie mit dem Instrument der vorwettbewerblichen Beschaffung und im Rahmen der innovationsfördernden öffentlichen Beschaffung

* Prüfung und ggf. Ausweitung des österreichischen Förderportfolios in Hinblick auf eine durchgängige Unterstützung von der Forschungs- über die Demonstrationsphase hin zur effektiven Kapitalisierung der Forschungsergebnisse (Nachverfolgung der Projekte, Zusammenarbeit mit Multiplikatoren, Förderung der internationalen Markterschließung wie bspw. im Pilotprogramm „aws tec4market", Unterstützung von Spin-Offs aus Forschungseinrichtungen uvam.)

* Antizipatives und strukturiertes Screening von internationalen Trends im Bereich der Bauwirtschaft im Allgemeinen und des Nachhaltigen Bauens im Besonderen zur frühzeitigen Identifikation von relevanten Entwicklungen mit hohem Marktpotenzial. Dabei sollte ein Schwerpunkt auf die stark wachsenden Schwellenländer („Emerging Markets“) und ihre diesbezüglichen Strategien und Programme gelegt werden. Obgleich der Gebäudebereich auch in Zukunft von hoher Relevanz sein wird, so wäre doch ein strukturiertes Trendscreening sinnvoll, um neue Zukunftsthemen frühzeitig erkennen und berücksichtigen zu können. 


\section{Literatur- und Quellenverzeichnis}

AEE INTEC - Institut für Nachhaltige Technologien (2008). Ergebnisse der messtechnischen Begleituntersuchungen von "Haus der Zukunft“ - Demonstrationsbauten.

Austrian Energy Agency [AEA] im Auftrag des Bundesministeriums für Verkehr, Innovation und Technologie (2015). Energieforschungserhebung 2014 Ausgaben der öffentlichen Hand in Österreich. Erhebung für die IEA.

Bundesimmobiliengesellschaft [BIG] (2015). Gemeinsam die Besten - Holistic Building Program der Bundesimmobiliengesellschaft.

Bundesministerium für Verkehr, Innovation und Technologie [BMVIT] (2000). Haus der Zukunft - Leitfaden 2. Ausschreibung im Rahmen des Impulsprogramms Nachhaltig Wirtschaften.

Bundesministerium für Verkehr, Innovation und Technologie [BMVIT] (2002a). Haus der Zukunft - Leitfaden 3. Ausschreibung im Rahmen des Impulsprogramms Nachhaltig Wirtschaften.

Bundesministerium für Verkehr, Innovation und Technologie [BMVIT] (2002b). Altbau der Zukunft - Preisträger, Auszeichnungen, Anerkennungen des Wettbewerbs 2001.

Bundesministerium für Verkehr, Innovation und Technologie [BMVIT] (2003). Haus der Zukunft - Leitfaden 4. Ausschreibung im Rahmen des Impulsprogramms Nachhaltig Wirtschaften.

Bundesministerium für Verkehr, Innovation und Technologie [BMVIT] (2004a). Impulsprogramm Nachhaltig Wirtschaften - Zwischenbilanz 2004.

Bundesministerium für Verkehr, Innovation und Technologie [BMVIT] (2004b). Aktuelle Ergebnisse Haus der Zukunft - Neubau.

Bundesministerium für Verkehr, Innovation und Technologie [BMVIT] (2005). Haus der Zukunft - Leitfaden 5. Ausschreibung im Rahmen des Impulsprogramms Nachhaltig Wirtschaften.

Bundesministerium für Verkehr, Innovation und Technologie [BMVIT] (2008). Haus der Zukunft plus - Leitfaden für Projekteinreichung 1. Ausschreibung.

Bundesministerium für Verkehr, Innovation und Technologie [BMVIT] (2009a). Haus der Zukunft plus - Leitfaden für Projekteinreichung 2. Ausschreibung. 
Bundesministerium für Verkehr, Innovation und Technologie [BMVIT] (2009b). 10 Jahre Programmlinie Haus der Zukunft 1999-2009.

Bundesministerium für Verkehr, Innovation und Technologie [BMVIT] (2010). Haus der Zukunft plus - 2. Ausschreibung 2010 Demonstrationsprojekte, Ergänzung zum Leitfaden für Projekteinreichung der 2. Ausschreibung 2009.

Bundesministerium für Verkehr, Innovation und Technologie [BMVIT] (2011). Haus der Zukunft plus - Leitfaden für Projekteinreichung 3. Ausschreibung.

Bundesministerium für Verkehr, Innovation und Technologie [BMVIT] (2012a). Haus der Zukunft plus - Leitfaden für Projekteinreichung 4. Ausschreibung.

Bundesministerium für Verkehr, Innovation und Technologie [BMVIT] (2012b). Evaluierung des Programms IEA-Forschungskooperation. Berichte aus Energie- und Umweltforschung Nr. 46/2012.

Bundesministerium für Verkehr, Innovation und Technologie [BMVIT] (2012c). Innovative Gebäude in Österreich - Österreichische Demonstrationsgebäude und Leitprojekte aus dem Forschungsprogramm "Haus der Zukunft".

Bundesministerium für Verkehr, Innovation und Technologie [BMVIT] (2012d). Bewertungshandbuch zur Begutachtung von Förderungs- und Finanzierungsansuchen im Rahmen der 4. Ausschreibung "Haus der Zukunft plus“.

Bundesministerium für Verkehr, Innovation und Technologie [BMVIT] (2013). Haus der Zukunft plus Vernetzungsworkshop für Projekte der 4. Ausschreibung am 21. Oktober 2013.

Bundesministerium für Verkehr, Innovation und Technologie [BMVIT] (2014). Programmdokument Energieforschung 2020 - Formale Laufzeit bis 31.12.2014.

Bundesministerium für Verkehr, Innovation und Technologie/Klima- und Energiefonds (2014). Gebäudeinnovationen aus Österreich im arabischen Raum Projekte und Perspektiven. energy innovation austria, Nr. 3/2014.

Bundesministerium für Verkehr, Innovation und Technologie [BMVIT] (2015). Programmdokument Energieforschung 2020 - Laufzeit bis 31.12.2020.

Bundesministerium für Verkehr, Innovation und Technologie/Bundesministerium für Wirtschaft und Arbeit (2007). Programmdokument Forschungs- und Technologieprogramm Energie der Zukunft, Periode 2007 - 2010. 
Bundesministerium für Wissenschaft, Forschung und Wirtschaft [BMWFW] (2014). Cluster in Österreich - Leistungsstarke Innovationsnetzwerke in den wirtschaftlichen und technologischen Stärkefeldern Österreichs.

Bundesministerium für Wissenschaft und Verkehr (1999). Haus der Zukunft - Leitfaden 1. Ausschreibung im Rahmen des Impulsprogramms Nachhaltig Wirtschaften - at:sd.

Bundesministerium für Wissenschaft und Forschung/Bundesministerium für Verkehr, Innovation und Technologie/Bundesministerium für Wirtschaft und Arbeit (2008). Österreichischer Forschungsund Technologiebericht 2008 - Lagebericht gem. $\S 8$ (1) FOG über die aus Bundesmitteln geförderte Forschung, Technologie und Innovation in Österreich [FTB].

Europäische Kommission - GD Forschung (2013). Energy-Efficient Buildings - Multiannual roadmap for the contractual PPP under Horizon 2020.

Europäische Kommission [EU-KOM] (2010). Europa 2020 - Eine intelligentes, nachhaltiges und integratives Wachstum. $\operatorname{KOM(2010)} 2020$.

Europäische Kommission [EU-KOM] (2011). Ressourcenschonendes Europa - eine Leitinitiative innerhalb der Strategie Europa 2020. KOM(2011) 21.

Europäische Kommission [EU-KOM] (2015). Towards an Integrated Strategic Energy Technology (SET) Plan - Accelerating the European Energy System Transformation.

Europäische Kommission [EU-KOM] (2015). Paket zur Energieunion - Rahmenstrategie für eine krisenfeste Energieunion mit einer zukunftsorientierten Klimaschutzstrategie, $\operatorname{COM}(2015) 80$ final.

Institut für Immobilien, Bauen und Wohnen [IIBW] (2013). Bestandsaufnahme der österreichischen Baulandschaft - Stadtmorphologien.

Mandl, Lüthi \& Partner (2010a). Strategisches Monitoring des Programms Haus der Zukunft Plus - Zwischenbericht I. Eine Arbeit im Auftrag des Bundesministeriums für Verkehr, Innovation und Technologie.

Mandl, Lüthi \& Partner (2010b). Strategisches Monitoring des Programms Haus der Zukunft Plus - Zwischenbericht II. Eine Arbeit im Auftrag des Bundesministeriums für Verkehr, Innovation und Technologie.

Mandl, Lüthi \& Partner (2011). Strategisches Monitoring des Programms Haus der Zukunft Plus - Endbericht. Eine Arbeit im Auftrag des Bundesministeriums für Verkehr, Innovation und Technologie. 
Österreichische Bundesregierung (2011). Der Weg zum Innovation Leader - Strategie der Bundesregierung für Forschung, Technologie und Innovation.

Österreichische Gesellschaft für Umwelt und Technik [ÖGUT] (2007a). Endbericht der Arbeitsgruppe Haus der Zukunft in der ÖGUT - Juli 2004 bis April 2007.

Österreichische Gesellschaft für Umwelt und Technik [ÖGUT] (2007b). Tätigkeitsbericht der Arbeitsgruppe Haus der Zukunft in der ÖGUT September bis November 2007.

Österreichische Gesellschaft für Umwelt und Technik [ÖGUT] (2007c). Endbericht der Arbeitgruppe "Haus der Zukunft" in der ÖGUT April 2006 bis August 2007.

Österreichische Gesellschaft für Umwelt und Technik [ÖGUT] (2010). Unterstützung der Arbeiten zum Programm Haus der Zukunft Plus - Endbericht vom Dezember 2010.

Österreichische Gesellschaft für Umwelt und Technik [ÖGUT] (2011a). Unterstützung der Öffentlichkeitsarbeit zum Programm Haus der Zukunft Plus - Endbericht vom Juli 2011.

Österreichische Gesellschaft für Umwelt und Technik [ÖGUT] (2011b). Unterstützung der strategischen Arbeit und Projektbegleitung zum Programm Haus der Zukunft Plus - Endbericht vom September 2011.

Österreichische Gesellschaft für Umwelt und Technik [ÖGUT] (2011c). Internationale Programmunterstützung der Arbeiten zum Programm Haus der Zukunft Plus - Endbericht vom Juli 2011.

Österreichische Gesellschaft für Umwelt und Technik [ÖGUT] (2014a). Unterstützungsleistungen zum Programm Haus der Zukunft plus - Endbericht vom April 2014.

Österreichische Gesellschaft für Umwelt und Technik [ÖGUT] (2014b). Diskussion eines „Austrian Standard“ für Gebäude und Entwicklung des Forschungsbereichs „Urbane Technologien.“

Österreichisches Ökologie-Institut / Österreichisches Institut für Bauen und Ökologie [Hrsg.] (2015). Nachhaltig Bauen in Österreich - Weißbuch 2015.

Salzburger Institut für Raumordnung und Wohnen [SIR] (2010). StadtUMBAU Lehen. 
Programmevaluierung Haus der Zukunft 1999 - 2013

Schneider, Friedrich / Voigt, Jasmin (2016). Volkswirtschaftliche Analyse der regionalen Wertschöpfungs- und Beschäftigungseffekte durch das Programm „Haus der Zukunft“ bzw. „Haus der Zukunft Plus“. 


\section{Abbildungs- und Tabellenverzeichnis}

\section{Abbildungsverzeichnis:}

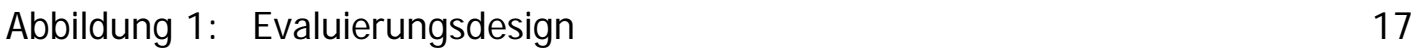

Abbildung 2: Ziele des Impulsprogramms Nachhaltig Wirtschaften 20

Abbildung 3: Programmlinien des Impulsprogramms Nachhaltig Wirtschaften 21

Abbildung 4: Anteil der Energieforschung an den Bruttoinlandsausgaben (Bund und Länder) für $F \& E$

Abbildung 5: Energieforschungsausgaben im Themenfeld "Gebäude und Geräte“ von allen Einrichtungen 2005 - 2014

Abbildung 6: Gebäuderelevante Energieforschungsausgaben von allen Einrichtungen 2005 - 2014

Abbildung 7: Energieforschungsausgaben der Bundesländer 2010 - $2014 \quad 45$

Abbildung 8: Projektanträge und -genehmigungen Haus der Zukunft 49

Abbildung 9: Projektanträge und -genehmigungen Haus der Zukunft plus 49

Abbildung 10: Eingereichte und genehmigte Beteiligungen Haus der Zukunft (ab 3. AS)

Abbildung 11: Eingereichte und genehmigte Beteiligungen Haus der Zukunft plus50

Abbildung 12: Genehmigte Gesamtkosten / Förderung Haus der Zukunft plus 53

Abbildung 13: Förderung nach Jahr der Genehmigung

Abbildung 14: Beteiligte an genehmigten Projekten nach Organisationstyp Haus der Zukunft

Abbildung 15: Beteiligte an genehmigten Projekten nach Organisationstyp Haus der Zukunft plus

Abbildung 16: Genehmigte Förderung nach Organisationstyp Haus der Zukunft 56

Abbildung 17: Genehmigte Förderung nach Organisationstyp Haus der Zukunft plus

Abbildung 18: Beteiligte Unternehmen an geförderten Projekten nach NACE-

Klassen

Abbildung 19: Genehmigte Gesamtkosten und Förderung nach Bundesländern Haus der Zukunft

Abbildung 20: Genehmigte Gesamtkosten und Förderung nach Bundesländern Haus der Zukunft plus 
Abbildung 21: Landkarte Demonstrationsprojekte

Abbildung 22: Leitprojekte, Subprojekte und verbundene

Demonstrationsprojekte

Abbildung 23: Um welche Art von Projekten handelte es sich dabei?

(Mehrfachnennungen möglich)

Abbildung 24: Welche Auswirkung hatte das Projekt unmittelbar auf das Unternehmen? Bitte geben Sie auf der Skala an, was durch das Projekt in Ihrem Unternehmen bewirkt wurde!

Abbildung 25: Wurde im Zuge des Projektes ein neues Produkt, eine neue Technologie oder ein neues Dienstleistungsangebot erarbeitet?

Abbildung 26: Wie erfolgreich war die Marktakzeptanz dieses neuen Angebotes?92

Abbildung 27: Konnten aufgrund der Forschungs- und Innovationsaktivitäten neue Arbeitsplätze geschaffen werden?

Abbildung 28: Haben Sie nach Abschluss des geförderten Projektes weiter an dem Thema gearbeitet?

Abbildung 29: In welcher Form? (Mehrfachnennungen möglich)

Abbildung 30: Was konnte nach Ihrer Wahrnehmung durch das Programm Haus der Zukunft insgesamt in Österreich bewirkt werden? Bitte geben Sie auf der Skala an, wie hoch Sie die Wirkung einschätzen!

Abbildung 31: Wie wichtig waren nach Ihrer Meinung die begleitenden Angebote des Programms Haus der Zukunft?

Abbildung 32: Wie relevant sind folgende Themenfelder für die künftigen Forschungs- und Innovationsaktivitäten? Bitte geben Sie auf der Skala die Relevanz dieser Themenfelder für Ihr Unternehmen und für den Forschungs- und Wirtschaftsstandort Österreich insgesamt an!

Abbildung 33: Was ist erforderlich, damit Sie diese Forschungs- und Innovationsaktivitäten erfolgreich umsetzen können? (Mehrfachnennungen möglich)

Abbildung 34: An welcher bzw. welchen Ausschreibung(en) des Förderprogramms Haus der Zukunft haben Sie teilgenommen? (Mehrfachnennungen möglich)

Abbildung 35: Hätte Ihre Einrichtung auch ohne die Förderung an diesem Thema geforscht?

Abbildung 36: Welche Auswirkung hatte das Projekt unmittelbar auf I hre Einrichtung? Bitte geben Sie auf der Skala an, was durch das Projekt in Ihrem Unternehmen bewirkt wurde! 
Abbildung 37: Haben Sie nach Abschluss des geförderten Projektes weiter an dem Thema gearbeitet? Wenn ja, in welcher Form?

Abbildung 38: Was konnte nach Ihrer Wahrnehmung durch das Programm Haus der Zukunft insgesamt in Österreich bewirkt werden? Bitte geben Sie auf der Skala an, wie hoch Sie die Wirkung einschätzen!

Abbildung 39: Wie wichtig waren nach Ihrer Meinung die begleitenden Angebote des Programms Haus der Zukunft?

Abbildung 40: Wie relevant sind folgende Themenfelder für die künftigen Forschungs- und Innovationsaktivitäten? Bitte geben Sie auf der Skala die Relevanz dieser Themenfelder für Ihr Unternehmen und für den Forschungs- und Wirtschaftsstandort Österreich insgesamt an!

Abbildung 41: In welcher Form planen Sie, sich mit diesen Themenfeldern auseinanderzusetzen?

Abbildung 42: Was ist erforderlich, damit Sie diese Forschungs- und Innovationsaktivitäten erfolgreich umsetzen können?

Abbildung 43: Wie wichtig waren nach Ihrer Meinung die begleitenden Angebote des Programms "Haus der Zukunft"?

Abbildung 44: Flussdiagramm des Simulationsmodells

Abbildung 45: Was konnte nach Ihrer Wahrnehmung durch das Programm „Haus der Zukunft" insgesamt in Österreich bewirkt werden? Bitte geben Sie auf der Skala an, wie hoch Sie die Wirkung einschätzen!124

\section{Tabellenverzeichnis:}

Tabelle 1: $\quad$ Übersicht der Programminhalte

Tabelle 2: $\quad$ Beantragte und genehmigte Gesamtkosten bzw. Förderung im Überblick

Tabelle 3: $\quad$ Übersicht Leitprojekte 